\title{
Characterisation, optimisation and performance studies of pixel vertex detector modules for the Belle II experiment
}

\author{
Dissertation
}

zur Erlangung des mathematisch-naturwissenschaftlichen Doktorgrades "Doctor rerum naturalium“

der Georg-August-Universität Göttingen

im Promotionsprogramm ProPhys

der Georg-August University School of Science (GAUSS)

vorgelegt von

Philipp Wieduwilt

aus Stade

Göttingen, 2021 
Betreuungsausschuss

Prof. Dr. Ariane Frey

PD Dr. Jörn Große-Knetter

Mitglieder der Prüfungskommission:

Referentin: Prof. Dr. Ariane Frey

II. Physikalisches Institut, Georg-August-Universität Göttingen

Koreferent: PD Dr. Jörn Große-Knetter

II. Physikalisches Institut, Georg-August-Universität Göttingen

Weitere Mitglieder der Prüfungskommission:

Prof Dr. Stan Lai

II. Physikalisches Institut, Georg-August-Universität Göttingen

Prof. Dr. Hans Christian Hofsäss

II. Physikalisches Institut, Georg-August-Universität Göttingen

Prof. Dr. Baida Achkar

II. Physikalisches Institut, Georg-August-Universität Göttingen

Prof. Dr. Wolfram Kollatschny

Institut für Astrophysik, Georg-August-Universität Göttingen

Tag der mündlichen Prüfung: 03.05.2021

Referenz: II.Physik-UniGö-Diss-2021/02 


\title{
Characterisation, optimisation and performance studies of pixel vertex detector modules for the Belle II experiment
}

\begin{abstract}
The Standard Model of particle physics is very successful in describing the fundamental particles and their interactions. Still, some questions regarding the details and input parameters of the theory, as well as regarding to date unsatisfactorily described phenomena are to be answered. Today's high-energy physics experiments probe this new physics. The Belle II experiment at the SuperKEKB $e^{+} e^{-}$-collider in Japan explores the precision frontier, measuring the properties of particle interactions at great detail. The precise and abundant study of decays of $B$-mesons is a particularly good window to seek answers to the open questions of electro-weak interactions. Precise measurements in Belle II are possible in particular with a silicon pixel detector located very close to the interaction region of the electrons and positrons. The pixel detector is based on the depleted field-effect transistor (DEPFET) technology, which is employed for the first time in a high-energy physics experiment.

Modules for the Belle II pixel detector were produced. A characterisation and optimisation procedure for these modules was developed in the scope of this thesis. A number of 17 modules have been processed according to this program, and the qualification criteria for the installation in Belle II have been determined. In addition, the performance of detector modules has been evaluated in beam tests. It is demonstrated that the optimisation procedure yields consistent characteristics among the tested modules. Signal-to-noise ratios of 20 to 40 are achieved at an in-pixel amplification factor of about $500 \mathrm{pA} /$ electron in the DEPFET cell. The intrinsic spatial resolution is measured to be in the order of $10 \mu \mathrm{m}$, depending on pixel pitch and incidence angles, with a hit efficiency of $99.6 \%$. The module performance is in good agreement with the design goals and the requirements for the Belle II pixel detector are met.
\end{abstract}





\section{Characterisation, optimisation and performance studies of pixel vertex detector modules for the Belle II experiment}

\section{Zusammenfassung}

Das Standardmodell der Elementarteilchenphysik beschreibt sehr erfolgreich die fundamentalen Teilchen und ihre Wechselwirkungen. Dennoch bleiben einige Fragen bezüglich Details und Parameter der Theorie bislang unbeantwortet. Einige Phänomene, wie beispielsweise Dunkle Materie oder Quantengravitation, sind bisher nicht, oder nur unzureichend beschrieben. Heutige Hochenergiephysikexperimente erforschen diese neue Physik in Teilchenkollisionen. Das Belle II-Experiment am SuperKEKB $e^{+} e^{-}$-Beschleuniger in Japan untersucht die Eigenschaften von Teilchenwechselwirkungen mit höchster Präzision, um so die Grenzen der bisherigen Theorie zu erweitern und Parameter des Standardmodells genauer zu bestimmen. Die genaue Beobachtung von zahlreichen Zerfällen von $B$-Mesonen ermöglicht es, offene Fragen der elektroschwachen Wechselwirkung zu beantworten. Präzisionsmessungen im Belle II Experiment werden insbesondere durch einen Silizium-Pixeldetektor ermöglicht, der sehr nah am Wechselwirkungspunkt der Teilchenkollisionen positioniert ist. Der Pixeldetektor basiert auf der depleted field-effect transistor (DEPFET) Technologie, die hier zum ersten Mal in einem Hochenergiephysikexperiment zum Einsatz kommt.

Für den Belle II Pixeldetektor wurden auf dieser Technologie basierende Module produziert. Eine genaue Charakterisierungs- und Optimierungsprozedur für diese Module wurde im Rahmen dieser Dissertation entwickelt. Insgesamt 17 dieser Module wurden diesem Messprogramm im Verlauf dieser Arbeit unterzogen und die Qualifikation jedes einzelnen Moduls für den finalen Detektor wurde geprüft. Detailierte Untersuchungen des Verhaltens der Detektormodule wurden in Teststrahlmessungen durchgeführt. Die Optimierungsprozedur liefert konsistente Resultate zwischen den getesteten Modulen mit Signal-Rausch-Verhältnissen im Bereich von 20 bis 40 bei einem InpixelVerstärkungsfaktor von etwa $500 \mathrm{pA} /$ electron. Die intrinsische Ortsauflösung der Detektormodule wurde zu etwa $10 \mu \mathrm{m}$ gemessen, abhängig vom Einfallswinkel der Teilchen, bei einer Detektionseffizienz von 99.6\%. Das gemessene Verhalten entspricht den Erwartungen des Moduldesigns und die Voraussetzungen an den Belle II Pixeldetektor werden mit dem vorliegenden Moduldesign erfüllt. 

1. Introduction 1

2. The Standard Model of Particle Physics 5

2.1. Elementary particles . . . . . . . . . . . . . . . . 5 5

2.2. Particle interactions . . . . . . . . . . . . . 6

2.3. The (electro-)weak force . . . . . . . . . . . . . . . 8

3. SuperKEKB - A Super B-factory 15

4. The Belle II Experiment - Precision B-physics 19

4.1. Belle II - A multi-purpose particle detector . . . . . . . . . . . . . . 20

4.2. Precision measurements and physics program . . . . . . . . . . 26

4.3. The importance of vertex resolution . . . . . . . . . . . . 27

5. Interaction of Charged Particles with Matter

- The Principles of Tracking Detectors 29

5.1. Energy loss in matter . . . . . . . . . . . . . . . . . 30

5.2. Silicon semiconductor detectors for particle tracking . . . . . . . . . 32

5.3. Spatial information in pixel detectors . . . . . . . . . . . . . . . 34

6. A DEPFET-based Pixel Detector for Belle II 35

6.1. The DEPFET operation principle . . . . . . . . . . . . . 36

6.2. Detector modules for Belle II . . . . . . . . . . . . . . . . . . 38

6.3. The Belle II pixel detector . . . . . . . . . . . . . . . . . . . . . 45

7. Series Testing of Pixel Detector Modules for Belle II 47

7.1. Production of Belle II pixel detector modules . . . . . . . . . . . . . . . . 47

7.2. Testing and characterisation procedure . . . . . . . . . . . 49

7.3. Optimisation of the DEPFET biasing . . . . . . . . . . . 63 
7.4. Results from the Göttingen test stand . . . . . . . . . . . . . . . 70

8. Measuring Pixel Detector Modules in Beam Tests 81

8.1. Beam test measurements at the DESY test beam facility . . . . . . . . . . 82

8.2. Devices under test and operation . . . . . . . . . . . . . 86

8.3. Data processing and track reconstruction . . . . . . . . . . . . 90

8.4. Module response to electron beam . . . . . . . . . . . . . . 100

8.5. Resolution studies . . . . . . . . . . . . . . . . . 106

8.6. Hit efficiency studies . . . . . . . . . . . . . . . . . . . 120

8.7. Sub-pixel resolved measurements . . . . . . . . . . . . . . 126

8.8. Influence of the DEPFET biasing . . . . . . . . . . . . . 131

$\begin{array}{ll}\text { 9. Summary and conclusion } & 137\end{array}$

$\begin{array}{lc}\text { Bibliography } & 141\end{array}$

$\begin{array}{ll}\text { Appendices } & 147\end{array}$

$\begin{array}{lr}\text { A. Spatially resolved hit efficiencies } & 149\end{array}$

$\begin{array}{ll}\text { B. DCD input gain dependence } & 153\end{array}$ 
CHAPTER 1

Introduction

The Standard Model of particle physics is a very successful theory, continuously validated and extended over the last decades. It is a quantum field theory describing the properties and interactions of elementary particles, the fundamental building blocks of the universe. Despite its success in explaining and predicting the outcomes of various measurements, it is still missing some key elements. Foremost, a consistent unification of the Standard Model with a complete quantum field theory of gravity is still to be found. Also, the nature of dark matter and how it embeds in the currently known "zoo of particles" is still unclear. There are also still open questions regarding the internals of the Standard Model itself.

Today's particle physics experiments push the limits of the Standard Model. Some operate at the energy frontier and are designated to explore new physics at higher energy scales. These could reveal new heavy particles as well as new or modified behaviour of particle interactions. Experiments of this type are for instance the ATLAS and CMS experiment at the Large Hadron Collider at CERN. Other experiments, like the Belle II experiment at the SuperKEKB collider at KEK, tackle the precision frontier. In a quantum field theory, phenomena more relevant at higher energy scales contribute to a small extent to phenomena at lower energy scales by loop corrections. For example, yet unobserved particles with high masses may contribute to observables at lower energy scales. Precise measurements of physics processes at well-known energy scales, resolving these loop correction contributions, hence facilitate conclusions on new physics phenomena as well.

The Belle II experiment in conjunction with the SuperKEKB accelerator is the successor of the Belle experiment with an up to forty-fold increase in instantaneous luminosity. It is designed to measure a very large data sample of $B$ meson decays with high precision. The pixel vertex detector of Belle II is an integral part for the reconstruction of $B$ meson decay events in the experiment. It is a silicon semi-conductor pixel detector based on 


\section{Introduction}

the depleted field-effect transistor (DEPFET) detection principle. The DEPFET based design enables to build thin detector devices with low material budget and low thermal power dissipation - a perfect choice for vertex detectors located very close to the interaction point of the accelerator. Belle II is the first particle physics experiment utilising DEPFET-based detectors.

Building a pixel detector with a few million read-out channels, on a centimetre scale, to be installed in a dense and spatially very limited location and operated in a harsh radiation environment is a very challenging task. The DEPFET pixel detector for Belle II is built from forty electrically independent modules. Each module is tested for basic functionality, characterised, and operation parameters are optimised in an extensive series testing program. The performance of single modules is measured in a controlled beam test environment and the realisation of certain design goals is demonstrated. The scope of this thesis is to define and conduct the series testing effort within the Belle II pixel detector collaboration. The characterisation and optimisation procedures are discussed and the results of the series testing share undertaken at the laboratory in Göttingen are presented. Beam test measurements are conducted at the DESY test beam facility and the essential performance figures of merit for a pixel detector - the spatial resolution and the hit efficiency — are determined.

Some words on notation Various symbols and mathematical notations are used in this thesis. Symbols used in multiple contexts are enhanced by subscripts and superscripts to distinguish specific results or use cases. For the chapters discussing the theoretical backgrounds, symbols are only briefly defined. In general, a widely understood usage of symbols as applied in many textbooks is adopted. Symbols used in the context of the specific pixel detector design, the conducted measurements and discussion of their results are thoroughly defined and explained on first usage. Best effort is given to keep the notation and usage of symbols consistent throughout the text.

Some general mathematical notation shall be defined here and is valid for the full text. The average of a quantity $x$ over a set of values is denoted with angle brackets $\langle x\rangle$. If not stated otherwise, the average is the arithmetic mean. If the average is weighted, the weighting is explicitly stated, as is the set over which the average is taken. When analysing the distribution of a certain quantity, the percentiles $P_{n}$ are used, where $n$ is a number in the range of 0 to 100 . The median of a distribution is labelled $P_{50}$. The symbols $i, j$ and $k$ usually denote integer indices enumerating a discrete set of quantities. In some cases the indices are omitted for readability, it should be clear from the context where indices are implied.

Units in table headers or axis labels are given in square brackets like [um]. Masses, energies and momenta are given in natural units and the speed of light $c=1$ is not explicitly spelled out. That means a mass of $10.58 \mathrm{GeV} / \mathrm{c}^{2}$ is written as $10.58 \mathrm{GeV}$.

Abbreviations are used abundantly. All acronyms used in this thesis are defined on first occurrence and the short forms are used subsequently. The meaning of certain abbreviations may be repeated occasionally - for example when used across chapters - to remind the reader. A table of acronyms is given on the following page. 


\begin{tabular}{|c|c|}
\hline acronym & meaning \\
\hline $\mathrm{ADC}$ & analogue-to-digital conversion/converter \\
\hline ADU & arbitrary digital unit \\
\hline $\mathrm{ARICH}$ & aerogel ring imaging Cherenkov detector (of Belle II) \\
\hline ASIC & application-specific integrated circuit \\
\hline $\mathrm{CDC}$ & central drift chamber \\
\hline CKM & Cabibbo-Kobayashi-Maskawa (quark mixing matrix) \\
\hline (A) $\mathrm{CMC}$ & (analogue) common mode correction \\
\hline CMOS & complementary metal-oxide-semiconductor \\
\hline COG & centre of gravity \\
\hline CSA & cluster shape algorithm \\
\hline $\mathrm{DAC}$ & digital-to-analogue conversion/converter \\
\hline DAQ & data acquisition software \\
\hline $\mathrm{DCD}$ & Drain Current Digitizer \\
\hline DEPFET & depleted field-effect transistor \\
\hline $\mathrm{DHC}$ & Data Handling Collector \\
\hline DHE & Data Handling Engine \\
\hline $\mathrm{DHH}$ & Data Handling Hybrid \\
\hline DHP & Data Handling Processor \\
\hline DUT & device under test \\
\hline ECL & electromagnetic calorimeter (of Belle II) \\
\hline EOS & end-of-stave (region of a Belle II pixel detector module) \\
\hline FET & field-effect transistor \\
\hline FPGA & field-programmable gate array \\
\hline FWHM & full width at half maximum \\
\hline HER & high-energy ring $\left(e^{-}\right.$ring of SuperKEKB) \\
\hline IP & interaction point $\left(e^{+} e^{-}\right.$collision point of SuperKEKB) \\
\hline JTAG & Joint Test Action Group (IEEE Standard 1149.1) \\
\hline KLM & $K_{L}$ and muon detector (of Belle II) \\
\hline LER & low-energy ring ( $e^{+}$ring of SuperKEKB) \\
\hline MOS & metal-oxide-semiconductor \\
\hline MOSFET & metal-oxide-semiconductor field-effect transistor \\
\hline MPV & most-probable value (of a Landau distribution) \\
\hline PCB & printed circuit board \\
\hline PFA & position finding algorithm \\
\hline PID & particle identification system (of Belle II) \\
\hline PMT & photo multiplier tube \\
\hline PXD & pixel detector (of Belle II) \\
\hline RMS & root-mean-square \\
\hline $\mathrm{RPC}$ & resistive plate chamber \\
\hline $\mathrm{SM}$ & the Standard Model of particle physics \\
\hline SNR & signal-to-noise ratio \\
\hline SVD & silicon (strip) vertex detector (of Belle II) \\
\hline TLU & trigger logic unit \\
\hline TOP & time-of-propagation detector (of Belle II) \\
\hline VXD & vertex detector (of Belle II) \\
\hline
\end{tabular}



CHAPTER 2

\section{The Standard Model of Particle Physics}

\subsection{Elementary particles}

The field of particle physics covers the study of elementary particles, their properties and the nature of their interactions and the mechanisms forming compound particles. A particle is considered elementary if it does not possess any sub-structure and spatial extent (i.e. it is point-like). The most familiar elementary particle, and indeed the first one discovered as such in 1897 by J. J. Thomson [1], is the electron. In the scope of the 20th century, many more elementary particles were discovered and grouped according to common properties like charge and spin. The muon ( $\mu$ discovered in 1936 [2]) and the tau ( $\tau$ discovered in 1975 [3]) are heavier versions of the electron. These three spin $1 / 2$ particles with an electric charge of -1 are called charged leptons. Each has a neutral (electric charge of 0) and very light-weight spin 1/2 neutrino partner $\left(\nu_{\mathrm{e}}, \nu_{\mu}\right.$ and $\left.\nu_{\tau}\right)$, they are collectively called neutral leptons. Together, charged and neutral leptons form the three lepton generations. Due to their half-integer spin, these particles belong to the fermions. Fermions are particles (elementary or compound) that respect the Fermi-Dirac statistic.

Another class of fermions, the quarks, were discovered to be the constituents of compound particles like the proton or neutron, that in turn form the atomic nuclei. Like the charged leptons, quarks have a spin of $1 / 2$ and carry an electric charge. However, the quarks' electric charge is a fraction of the elementary charge, namely $-1 / 3$ or $+2 / 3$. Additionally, quarks carry another type of charge, the so called colour charge. Due to the nature of their interaction, quarks cannot be observed individually ${ }^{1}$, unlike leptons, but always exist in a bound state called hadron. In the same way as the leptons, quarks are arranged in three generations with increasing mass. Each generation has an up-type

\footnotetext{
${ }^{1}$ The exception is the top quark. With a lifetime of $\tau_{t}=3.29 \times 10^{-25} \mathrm{~s}$ [4], the top quark decays before a bound state can form.
} 


\section{The Standard Model of Particle Physics}

quark (electric charge of $+2 / 3$ ) and a down-type quark (electric charge of $-1 / 3$ ). The quarks are called up and down (first generation), charm and strange (second generation) and top and bottom (third generation), usually denoted by their first letter. Each lepton and each quark has a corresponding anti-particle with identical mass, but opposite additive quantum numbers such as the electric charge. Depending on the quark content, hadrons are categorised in baryons, consisting of three quarks $(q q q)^{2}$, and mesons, consisting of a quark-anti-quark pair $(q \bar{q})$.

Besides the fermions, another class of elementary particles exists: bosons. These particles respect the Bose-Einstein statistics and have an integer valued spin. A special group of bosons, the gauge bosons, are particles that mediate the interaction between leptons and quarks. Table 2.1 list the elementary particles, fermions and bosons, with their mass, electric charge, whether they carry a colour charge, the third component of the weak isospin for the left-handed fermions (see Sec. 2.3) and the spin.

\subsection{Particle interactions}

There are four types of forces acting on elementary particles: the electromagnetic force, the weak force, the strong force and the gravitational force. The former three are consistently embedded in the Standard Model of particle physics (SM), a renormalisable quantum field theory. As of yet, there is no consistent renormalisable quantum field theory of gravitation, the gravitational force is therefore not included in the SM. At the length, energy and mass scales considered in particle physics interactions, the gravitational force is expected to be negligibly small compared to the other three forces.

The electromagnetic force causes interactions of particles which carry a non-zero electric charge. The mediating boson is the massless spin 1 photon, usually denoted as $\gamma$. The strength of the electromagnetic force scales, also in sign, with the amount and the sign of the electric charge of the interacting particles. All quarks and the charged leptons interact electromagnetically.

The strong force couples particles which carry a colour charge. The mediating boson is the massless spin 1 gluon, usually denoted as $g$. In fact, overall eight different gluons exist. In contrast to the photon, the gluon itself carries a colour charge and therefore gluon self-interaction is possible. Only the quarks (and the gluons) interact via the strong force. As a consequence of the gluon self-interaction, two strongly interacting quarks experience a constant force between them. When being pulled apart, the energy linearly increases until a new quark-anti-quark pair is formed instead of separating the two quarks. This property is called colour confinement and as a result, quarks (except for the top quark) cannot be observed individually, but only in bound states. The process of highly energetic bound quark states (hadrons) to form new, lower energetic hadrons (instead of simply splitting up into single quarks) is called hadronisation. Another consequence is that at high energy scales or, equivalently, low distances, the strength of the strong force is reduced, the quarks are asymptotically free.

\footnotetext{
${ }^{2}$ There have been observations and discoveries of tetraquark $(q q \overline{q q})$ states [5] and pentaquark $(q q q q \bar{q})$ states [6].
} 


\begin{tabular}{|c|c|c|c|c|c|}
\hline particle & mass $[\mathrm{MeV}]$ & electric charge & colour charge & weak isospin & spin \\
\hline \multicolumn{6}{|c|}{ Fermions - Leptons } \\
\hline$\nu_{e}$ & $<10^{-6}$ & 0 & - & $1 / 2$ & $1 / 2$ \\
\hline$e^{-}$ & 0.511 & -1 & - & $-1 / 2$ & $1 / 2$ \\
\hline$\nu_{\mu}$ & $<0.17$ & 0 & - & $1 / 2$ & $1 / 2$ \\
\hline$\mu^{-}$ & 105.66 & -1 & - & $-1 / 2$ & $1 / 2$ \\
\hline$\nu_{\tau}$ & $<18.2$ & 0 & - & $1 / 2$ & $1 / 2$ \\
\hline$\tau^{-}$ & 1776.8 & -1 & - & $-1 / 2$ & $1 / 2$ \\
\hline \multicolumn{6}{|c|}{ Fermions - Quarks } \\
\hline$u$ & 2.2 & $2 / 3$ & $\checkmark$ & $1 / 2$ & $1 / 2$ \\
\hline$d$ & 4.7 & $-1 / 3$ & $\checkmark$ & $-1 / 2$ & $1 / 2$ \\
\hline$c$ & 1280 & $2 / 3$ & $\checkmark$ & $1 / 2$ & $1 / 2$ \\
\hline$s$ & 96 & $-1 / 3$ & $\checkmark$ & $-1 / 2$ & $1 / 2$ \\
\hline$t$ & 173100 & $2 / 3$ & $\checkmark$ & $1 / 2$ & $1 / 2$ \\
\hline$b$ & 4180 & $-1 / 3$ & $\checkmark$ & $-1 / 2$ & $1 / 2$ \\
\hline \multicolumn{6}{|c|}{ Gauge Bosons } \\
\hline$\gamma$ & 0 & 0 & - & 0 & 1 \\
\hline$W^{ \pm}$ & 80390 & \pm 1 & - & $\mp 1$ & 1 \\
\hline$Z^{0}$ & 91190 & 0 & - & 0 & 1 \\
\hline$g$ & 0 & 0 & $\checkmark$ & 0 & 1 \\
\hline$H$ & 124970 & 0 & - & 0 & 0 \\
\hline
\end{tabular}

Table 2.1.: Elementary particles and their properties [4].

The weak force couples to particle flavours. Flavour refers to the "species" of a particle. There are six lepton flavours $\left(e, \mu, \tau, \nu_{\mathrm{e}}, \nu_{\mu}\right.$ and $\left.\nu_{\tau}\right)$ and six quark flavours $(u$, $d, c, s, t$ and $b)$. In contrast to the other two forces, the weak force has three massive mediating spin 1 bosons, the neutral $Z^{0}\left(m_{Z} \approx 91 \mathrm{GeV}\right)$ and the two charged $W^{ \pm}$ $\left(m_{W} \approx 80 \mathrm{GeV}\right)$. It is also the only force that can change the flavour of a particle (e.g. $\left.\nu_{e} \Rightarrow e^{-}\right)$in an interaction. Moreover, it exhibits violation of parity $(P)$ symmetry and charge-conjugation $(C)$ symmetry and indeed even violates the combined $C P$ symmetry. The corresponding "charge" of the weak interaction is called weak isospin. The weak interaction couples to all fermions.

Technically and mathematically, the three forces in the Standard Model are described by a renormalisable quantum field theory. The description of each interaction is based on a local gauge invariant Lagrangian with different gauge groups for the electromagnetic $(\mathrm{U}(1))$, the weak $(\mathrm{SU}(2))$ and the strong $(\mathrm{SU}(3))$ force. 


\subsection{The (electro-)weak force}

The weak interaction has some distinct features compared to the electromagnetic and the strong interaction since

- it is mediated by massive and electrically charged bosons $Z^{0}$ and $W^{ \pm}$,

- it can change the flavour of a particle and couples across quark generations (see section 2.3.1),

- and it violates parity symmetry $P$, charge-conjugation symmetry $C$ and the combined $C P$ symmetry.

Due to their large mass, the weak force bosons are short lived $\left(\tau_{Z, W}<10^{-24} \mathrm{~s}\right.$ [4] $)$ and usually only exist "virtually" in weak interactions. Allowing flavour changes, the weak interaction governs particle decays ${ }^{3}$, for example decays of heavy charged leptons $\tau^{-} \rightarrow e^{-}+\overline{\nu_{e}}+\nu_{\tau}$ to lighter leptons or the nuclear $\beta$-decay of the neutron ${ }^{4} n[u d d] \rightarrow$ $p[u u d]+e^{-}+\overline{\nu_{e}}$. In the lepton sector, flavour changes are limited to couplings within generations. For each lepton generation, a respective lepton number $L$ is defined, where $L_{e}=1$ is assigned to electrons and electron-neutrinos, $L_{e}=-1$ for positrons and electron-anti-neutrinos and analogously for the $\mu$ and $\tau$ leptons. Experimentally, the three lepton numbers $L_{e, \mu, \tau}$ are conserved in charged lepton interactions. With the observation of neutrino oscillations [7-9], the lepton number conservation of neutral leptons is broken.

The parity transformation $P$ is the inversion of all spatial coordinates $\vec{x} \rightarrow-\vec{x}$, that means converting a system into its mirror image. In general, the behaviour of a system is not expected to change when converted into its mirror image. The electromagnetic and strong interaction indeed are fully invariant under parity transformation. However, measurements of $\beta$-decays of cobalt isotopes ${ }^{60} \mathrm{Co}$ aligned in a uniform magnetic field, conducted by Wu et al. in 1956 [10], revealed that the weak interaction violates the parity symmetry. In fact, the flavour changing charged current weak interaction (mediated by the $W^{ \pm}$) is maximally parity violating. In technical terms of chirality ${ }^{5}$ : The charged current weak interaction couples only to left-handed fermions. Mathematically, the weak interaction is thus implemented as a $V-A$ (vector minus axial-vector) structure, where a pure vector part is parity-conserving (like in the electromagnetic and strong interaction), but the combination with the axial-vector breaks the parity symmetry. The $V-A$ structure, in principle, allows the combined $C P$ symmetry - the combined parity and charge-conjugation ${ }^{6}$ transformation - to be conserved. However, even the

\footnotetext{
${ }^{3}$ Except for the hadronisation of heavy hadrons to lighter hadrons.

${ }^{4}$ The neutron decay involves the flavour change of a down quark to an up quark.

${ }^{5}$ Chirality is a property of the spinor, the mathematical object describing a fermion. A spinor can be decomposed into a right-handed and left-handed chiral component. The physical quantity of helicity, which is the projection of the spin in the direction of travel of a particle, is related to chirality, both are identical in the limit of $E \gg m$.

${ }^{6}$ The charge-conjugation transformation $C$ inverts all additive quantum numbers of a particle, i.e. it transforms a particle into its corresponding anti-particle.
} 
$C P$ symmetry is violated as discussed in the following sections. Consequently, this means that the charged current weak interaction couples only to left-handed fermions and right-handed anti-fermions.

The neutral current weak interaction, mediated by the $Z^{0}$, does not change particle flavours. Unlike for the charged bosons, the $Z^{0}$ coupling is not fully violating parity symmetry. Couplings to both left-handed and right-handed particles (and anti-particles) are possible, although the respective coupling strengths are not equal. The reason for this apparent inconsistency is the fact that the observed $Z^{0}$ boson is not identical to the neutral gauge boson of the pure $S U(2)$ gauge group of the weak interaction. Instead, the neutral bosons $Z^{0}$ and $\gamma$ of the electromagnetic interaction are both a superposition - or a "mixture" - of the neutral gauge bosons of the pure $S U(2)$ and $U(1)$ gauge groups. It is thus more appropriate to view the $\gamma, Z^{0}$ and $W^{ \pm}$as the bosons of a mixed and unified $U(1) \times S U(2)$ gauge group and denote it the electro-weak interaction.

\subsubsection{Quark mixing and flavour oscillations}

The quark sector does not exhibit flavour conservation analogous to the lepton numbers. Indeed, interactions of the form $s \rightarrow u+W^{-}$do exist next to the expected $d \rightarrow u+W^{-}$ coupling, although with lower coupling strength and thus lower probability. An example is the observed decay of the $\Lambda$ baryon (quark content $u d s$ ) to a proton (quark content uud): $\Lambda^{0} \rightarrow p+e+\overline{\nu_{e}}$. In 1963, Cabibbo postulated weak universality, meaning that the weak coupling to all fermions is equally strong [11]. In order to solve the issue of "mixed generation couplings" with different coupling strengths for the quarks, he introduced the concept of a mixed quark sector. The weak eigenstates, determining the coupling of quarks to the weak bosons, are not identical to the mass eigenstates, describing propagating quarks and strong bound states. At that time, only the $u, d$ and $s$ quark were known. Cabibbo thus formulated a $2 \times 2$ mixing matrix relating the weak quark eigenstates $d^{\prime}$ and $s^{\prime}$ to the mass eigenstates $d$ and $s$ as

$$
\left(\begin{array}{l}
d^{\prime} \\
s^{\prime}
\end{array}\right)=\left(\begin{array}{cc}
\cos \theta_{C} & \sin \theta_{C} \\
-\sin \theta_{C} & \cos \theta_{C}
\end{array}\right)\left(\begin{array}{l}
d \\
s
\end{array}\right)
$$

with the Cabibbo mixing angle $\theta_{C} \approx 13^{\circ}$ [4].

The cross-generation coupling of quarks enables the interesting process of flavour oscillations or more specifically neutral meson mixing. The neutral kaon $K^{0}$ (quark content $d \bar{s}$ ), for example, can oscillate into its anti-particle $\bar{K}^{0}$ (quark content $\bar{d} s$ ) via second-order weak interactions. Since this process is reversible, a continuing oscillation between the two states $K^{0} \leftrightarrow \bar{K}^{0}$ can be observed.

\subsection{2. $C P$-violation and the Cabibbo-Kobayashi-Maskawa matrix}

After the surprising result of both $P$-violation and $C$-violation in the weak interaction, it was assumed that the combined charge-conjugation and parity symmetry $C P$ would at least be conserved. Experiments by Cronin and Fitch in 1964 [12], however, proved that even $C P$ symmetry is violated in weak interactions. The argument goes as follows: 


\section{The Standard Model of Particle Physics}

- Given that the parity eigenvalues of both $K^{0}$ and $\bar{K}^{0}$ are -1 and the chargeconjugation $C$ turns $K^{0}$ into $\bar{K}^{0}$ and vice versa, $C P$-eigenstates can be constructed as

$$
\left|K_{( \pm)}\right\rangle=\frac{1}{\sqrt{2}}\left(\left|K^{0}\right\rangle \pm\left|\bar{K}^{0}\right\rangle\right)
$$

where $K_{(+)}$has a $C P$-eigenvalue of -1 and $K_{(-)}$has a $C P$-eigenvalue of +1 .

- Neutral kaons mostly decay into two or three pions (combinations of $\pi^{0}$ and $\pi^{ \pm}$), where the $2 \pi$ final state has a $C P$-eigenvalue of +1 and the $3 \pi$ final state has a $C P$-eigenvalue of -1 . Assuming $C P$ symmetry is conserved in the weak interaction, only $K_{(+)} \rightarrow 3 \pi$ and $K_{(-)} \rightarrow 2 \pi$ decays are observable.

- A $K^{0}$ beam can be considered to consist of equal amounts of $K_{+}$and $K_{(-)}$. The decay of $K_{(-)} \rightarrow 2 \pi$ is fast due to the large phase space available for the final state. After a few metres, the beam thus consists of $K_{(+)}$only and consequently, only $3 \pi$ decays are expected.

- Actually observed is a small fraction of $2 \pi$ decays also after a long beam line. This is an evidence that $K_{(+)} \rightarrow 2 \pi$ decays occur, which violates $C P$ symmetry.

As a consequence, the long-lived kaons, $K_{L}$, are not purely $K_{(+)} C P$-eigenstates, but they have a small admixture of $K_{(-)}$

$$
\left|K_{L}\right\rangle=\frac{1}{\sqrt{1+|\epsilon|^{2}}}\left(\left|K_{(+)}\right\rangle+\epsilon\left|K_{(-)}\right\rangle\right)
$$

where $\epsilon \approx 2.228 \times 10^{-3}$ [4], and for the short-lived $K_{S}$ analogously. This observation of $C P$-violation in the mixing of neutral meson states is denoted indirect $C P$-violation. Besides the pure pion decays, the $K_{L}$ can also decay semi-leptonically via $K_{L} \rightarrow \pi^{+}+$ $e^{-}+\bar{\nu}_{e}$ or $K_{L} \rightarrow \pi^{-}+e^{+}+\nu_{e}$, which are $C P$-conjugate final states. With $C P$-symmetry conserved, both decays are equally probable. However, experiments show that the $K_{L}$ decay to a positron $e^{+}$is slightly more probable than the decay to an electron $e^{-}[4]$. The weak interaction thus clearly distinguishes between matter and anti-matter.

Also direct $C P$-violation is observed in weak decays. For $K$ mesons, direct $C P$ violation has been observed in the ratios of the branching fractions of $K_{L / S} \rightarrow \pi^{0} \pi^{0}$ and $K_{L / S} \rightarrow \pi^{+} \pi^{-}[13,14]$.

With the experimental evidence at hand, the $C P$-violating nature of the weak interaction had to be implemented in the theoretical framework. Kobayashi and Maskawa proposed that a complex phase in the quark mixing matrix would account for $C P$ violating weak processes [15]. The $2 \times 2$ Cabibbo-matrix (Eq. 2.1), however, cannot accommodate an irreducible complex phase. The mixing matrix needs to be at least of size $3 \times 3$. Indeed, by introducing the $3 \times 3$ Cabibbo-Kobayashi-Maskawa matrix (CKM matrix), the third quark generation was predicted long before the $b$ and the $t$ quarks were discovered and even the second generation $c$ quark was not yet known. The CKM 
matrix mixes the three down-type ${ }^{7}$ quarks $d, s$ and $b$ to the three weak eigenstates $d^{\prime}$, $s^{\prime}$ and $b^{\prime}$ as

$$
\left(\begin{array}{l}
d^{\prime} \\
s^{\prime} \\
b^{\prime}
\end{array}\right)=\left(\begin{array}{ccc}
V_{u d} & V_{u s} & V_{u b} \\
V_{c d} & V_{c s} & V_{c b} \\
V_{t d} & V_{t s} & V_{t b}
\end{array}\right)\left(\begin{array}{l}
d \\
s \\
b
\end{array}\right)
$$

where the element $V_{u b}$ for example describes the coupling of $b \rightarrow u+W^{-}$. Since the intra-generation couplings are strongest, the diagonal elements of the CKM matrix are expected to be close to 1 . Couplings spanning over two generations are weakest, that means the off-off-diagonal elements (e.g. $V_{u b}$ ) are expected to be very small. Recent results of measurements of the CKM matrix element values are given in (Eq. 2.5) [16].

$$
V=\left(\begin{array}{ccc}
0.97446 \pm 0.00010 & 0.22452 \pm 0.00044 & 0.00365 \pm 0.00012 \\
0.22438 \pm 0.00044 & 0.97359_{-0.00011}^{+0.00010} & 0.04214 \pm 0.00076 \\
0.00896_{-0.00023}^{+0.00024} & 0.04133 \pm 0.00074 & 0.999105 \pm 0.000032
\end{array}\right)
$$

The values of the CKM elements are purely experiment based, a circumstance considered unappealing in the Standard Model.

Assuming there are only three quark generations (or higher generations do not mix with the first three generations), the CKM matrix has to be unitary $V V^{\dagger}=1_{3 \times 3}$. That means

$$
\sum_{k}\left|V_{i k}\right|^{2}=\sum_{i}\left|V_{i k}\right|^{2}=1
$$

for all $i=u, c, d$ and $k=d, s, b$, which expresses the weak coupling universality. On the other hand, unitarity implies

$$
\sum_{k} V_{i k} V_{j k}^{*}=0
$$

for $i \neq j$. This relation can be interpreted as describing six triangles in the complex plane, often called unitarity triangles. Due to the expression of $C P$-violation via a complex phase in the CKM matrix, measurements of these unitarity triangles (lengths and angles) are handles for quantifying the $C P$-violation. Figure 2.1 is a graphical representation of a unitarity triangle with constraints given by various measurements as indicated. The CKM matrix is often expressed via the Wolfenstein parametrisation

$$
V=\left(\begin{array}{ccc}
1-\lambda^{2} / 2 & \lambda & A \lambda^{3}(\rho-i \eta) \\
-\lambda & 1-\lambda^{2} / 2 & A \lambda^{2} \\
A \lambda^{3}(1-\rho-i \eta) & -A \lambda^{2} & 1
\end{array}\right)+\mathcal{O}\left(\lambda^{4}\right)
$$

where $\lambda=\sin \theta_{C}$ and $\eta \neq 0$ is the irreducible complex phase causing $C P$-violation. The recent measurement results in terms of the Wolfenstein parameters ${ }^{8}$ are $\lambda=0.22453 \pm$

\footnotetext{
${ }^{7}$ Mixing of the down-type quarks is done by convention, the formalism could equally well be based on mixing of the up-type quarks.

${ }^{8}$ The reduced parameters are defined as $\bar{\rho}=\rho\left(1-\lambda^{2} / 2+\ldots\right)$ and $\bar{\eta}=\eta\left(1-\lambda^{2} / 2+\ldots\right)$.
} 


\section{The Standard Model of Particle Physics}

0.00044, $A=0.836 \pm 0.015, \bar{\rho}=0.122_{-0.017}^{+0.018}$ and $\bar{\eta}=0.355_{-0.011}^{+0.012}$ [4]. The neutral kaon mixing parameter $\epsilon$ is related to the Wolfenstein parameters as

$$
|\epsilon| \propto \eta(1-\rho+\text { const. }) .
$$

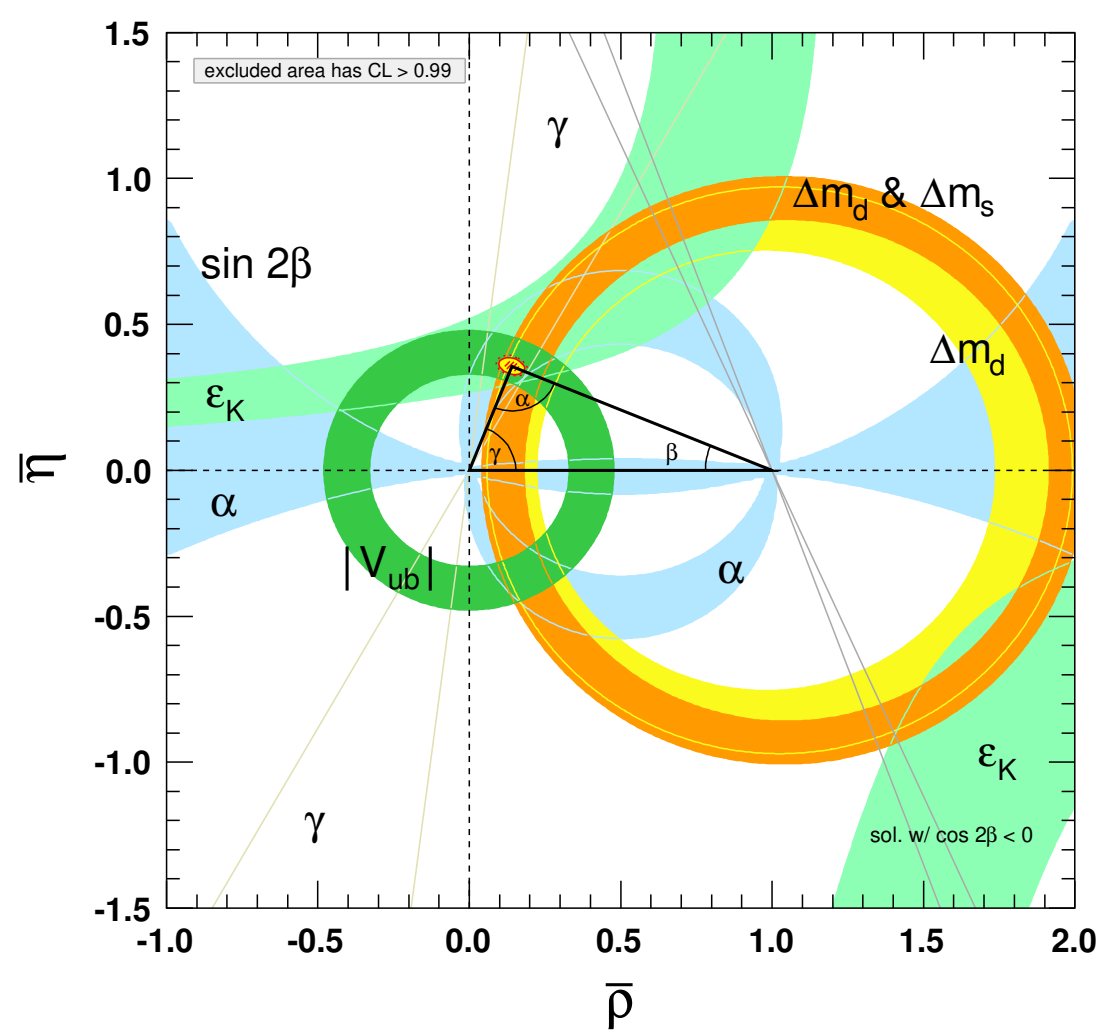

Figure 2.1.: Graphical representation of the unitarity triangle relevant for the $B$ meson system derived from the CKM matrix. Various measurements give constraints on angles or the length of the sides. Figure is from [4], where a detailed description of the individual constraints can be found.

\subsubsection{The $B$ meson system}

Neutral meson oscillations can also be observed in heavier mesons like the $B^{0}$ meson system

$$
B^{0}(\bar{b} d) \leftrightarrow \bar{B}^{0}(b \bar{d})
$$

with masses of $5.28 \mathrm{GeV}$ [4] each. Studies on oscillations and decays of $B$ mesons yield further information on the CKM matrix elements and $C P$-violation. The second-order weak interaction diagrams (called box diagrams) for the $B^{0} \leftrightarrow \bar{B}^{0}$ mixing are given in 

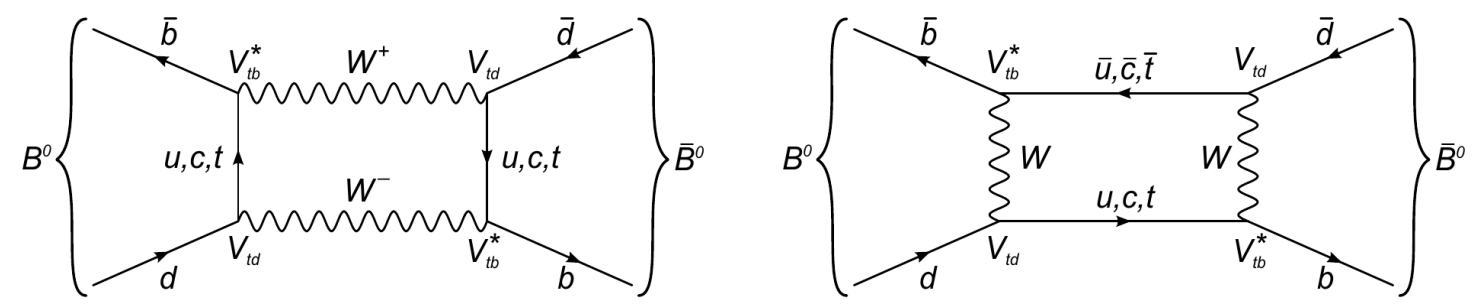

Figure 2.2.: Second-order weak interaction diagrams for the oscillation of the neutral $B$-mesons $B^{0} \leftrightarrow \bar{B}^{0}$ from [17].

Figure 2.2. The physical eigenstates of the neutral $B$ mesons can be constructed as

$$
\left|B_{ \pm}\right\rangle=\frac{1}{\sqrt{2}}\left(\left|B^{0}\right\rangle+e^{-i 2 \beta}\left|\bar{B}^{0}\right\rangle\right)
$$

only depending on the angle $\beta$. In contrast to the neutral kaon system, the differences in lifetimes of the two physical $B$-meson states is very small. The two states are thus usually denoted $\left|B_{+}\right\rangle=\left|B_{L}\right\rangle$ (light) and $\left|B_{-}\right\rangle=\left|B_{H}\right\rangle$ (heavy) due to their mass difference $\Delta m_{d} \approx \mathcal{O}\left(10^{-13}\right) \mathrm{GeV}$ [4]. Since the mass difference $\Delta m_{d}$ is proportional to the CKM matrix element $\left|V_{t d}\right|^{2}, B^{0} \leftrightarrow \bar{B}^{0}$ oscillation measurements can be used to constrain its value.

The effect of $C P$-violation can also be measured in the interference of neutral meson decays to a common final state, where the direct decay is compared to the decay with intermediate mixing by considering the asymmetry in the branching ratios for the $B^{0}$ and $\overline{B^{0}}$ initial states. In the $B$ meson system that means an interference of $B^{0} \rightarrow f$ and $B^{0} \rightarrow \bar{B}^{0} \rightarrow f$ decays, where the final state $f$ is for example the two meson state $J / \Psi$ and $K_{S}$. The time-dependent asymmetry between the two decay modes is a direct measurement of the mixing angle $\beta$ and the mass difference $\Delta m_{d}$

$$
A_{C P}^{K_{s}}=\frac{\Gamma\left(\bar{B}^{0} \rightarrow J / \Psi+K_{S}\right)-\Gamma\left(B^{0} \rightarrow J / \Psi+K_{S}\right)}{\Gamma\left(\bar{B}^{0} \rightarrow J / \Psi+K_{S}\right)+\Gamma\left(B^{0} \rightarrow J / \Psi+K_{S}\right)}=\sin \left(\Delta m_{d} t\right) \sin (2 \beta) .
$$

These measurements were performed at so called B-factories (see Chapter 3 ). The current world average results for the $B$ meson mixing observables are $\Delta m_{d}=3.33(2) 10^{-4} \mathrm{eV}$ and $\sin (2 \beta)=0.698(27)$ as given in [4]. Supported by many more measurements, the quark mixing model according to the CKM matrix is now established and was awarded with the Nobel Prize in 2008. 



\section{CHAPTER 3}

\section{SuperKEKB - A Super B-factory}

Electro-weak physics can be studied in high-energy charged particle collisions. Charged particles accelerated in electromagnetic fields and then collided produce new particles according to the energy-mass-equivalence $E=m c^{2}$. The spectrum of the created particles depends on the types of the colliding particles and, foremost, their energies. Circular particle accelerators are space-efficient (and thus cost-efficient) designs. Charged particles are kept on a circular trajectory by so-called bending magnets. Intermediate sections of cavities accelerate the particles in electric fields. In each turn, the particle's energy is increased due to the acceleration until the desired collision energies are reached. Due to the bent trajectory, synchrotron radiation is emitted tangentially to the accelerator. The energy loss due to the synchrotron radiation has to be compensated for by continuous acceleration. Circular particle colliders usually have two beam lines (double-ring) providing two beams of particles circulating in opposite directions. At one or more interaction points, the two beams are crossed and the particles collide. The key performance numbers of particle colliders are

- the collision energy $\sqrt{s}$, which is the centre of mass energy of the two colliding particles and

- the luminosity $\mathcal{L}$, which is the collision rate per interaction cross-section $\sigma$.

One type of circular particle collider, specifically tailored to study the electro-weak physics of $b$ quarks, are so called $B$-factories. They aim at investigating the effects of $C P$ violation and at observing rare particle decays of $B$-mesons in particular. These machines collide electrons $e^{-}$and positrons $e^{+}$at a centre of mass energy of $10.58 \mathrm{GeV}$ which is at the mass and thus the production resonance of the $\Upsilon(4 \mathrm{~S})$ meson (quark content $b \bar{b}$ ). The $\Upsilon(4 \mathrm{~S})$ decays predominantly to $B$-meson pairs (96\% branching ratio [16]) out of which $51.4 \%$ are charged $B^{+} B^{-}$and $48.6 \%$ are neutral $B^{0} \bar{B}^{0}$ pairs - hence the term 


\section{SuperKEKB - A Super B-factory}

$B$-factory. In the $\Upsilon(4 \mathrm{~S})$ decay, the two $B$-mesons with masses of $5.27 \mathrm{GeV}$ [16] are produced almost at rest (in the $\Upsilon(4 \mathrm{~S})$ centre of mass frame). For measurements of the time dependent $C P$-violation (cf. Eq. 2.12), a spatial separation of the $B$ decay vertices is required. A boost of the laboratory frame with respect to the centre of mass frame achieves this. Subsequent decay products can then be reconstructed to the two $B$ decay chains. The $B$-mesons have a mean lifetime of $\tau_{B^{0}}=1.52 \times 10^{-12} \mathrm{~s}$ and $\tau_{B^{ \pm}}=1.64 \times 10^{-12} \mathrm{~s}[16]$, respectively. An appropriately chosen boost results in mean travel lengths of a few $\mu \mathrm{m}$ up to $\mathrm{mm}$. Technically, this is accomplished by applying asymmetric energies to the two colliding beams.

Also the targeted and achieved high luminosity values in these colliders justify the term $B$-factories. High collision rates are realised and thus a large number of various $B$ meson decays can be measured and analysed. Since the effects of $C P$-violation are often small (see section 2.3.2), the reduction of statistical uncertainties in the measurements by maximising the available data is crucial. Two $B$-factories were successfully operated in the years 1999-2010 and delivered imported results for the electro-weak sector of the Standard Model. The PEP-II collider was operated at SLAC ${ }^{1}$ from 1999 to 2008 [18] providing the particle collisions for the BaBar experiment. The KEKB collider was operated at $\mathrm{KEK}^{2}$ from 1999 to 2010 and set a world record peak luminosity of $2.11 \cdot 10^{34} \mathrm{~cm}^{-2} \mathrm{~s}^{-1}$ in 2009 [18]. KEKB provided the particle collisions for the Belle experiment. Table 3.1 lists and compares some of the key parameters of the two machines.

In 2010, the construction of the upgrade of the KEKB machine, called SuperKEKB, was started. The design of the new machine targets a significantly increased instantaneous luminosity. First collisions with SuperKEKB were achieved in 2018. Compared to KEKB, SuperKEKB is planned to reach a 40 times higher peak instantaneous luminosity value and, over a life-time of 10 years, collect a 50 times larger integrated luminosity. This substantial increase is achieved, besides the increase of beam currents, by reducing the vertical and horizontal beam sizes down to a nano-metre scale, called "nano-beam scheme" [19]. Table 3.1 compares some of the important SuperKEKB performance parameters to the predecessor experiments. The boost of the centre of mass system at SuperKEKB is $\beta \gamma=0.283$ and thus lower than at KEKB $(\beta \gamma=0.425)$. SuperKEKB provides the particle collisions for the Belle successor experiment Belle II.

\footnotetext{
${ }^{1}$ Stanford Linear Accelerator Center, Menlo Park, USA

${ }^{2}$ High Energy Accelerator Research Organization, Tsukuba, Japan
} 


\begin{tabular}{lllll}
\hline parameter & PEP-II & KEKB & SuperKEKB & unit \\
\hline beam energies & $9.0\left(e^{-}\right), 3.1\left(e^{+}\right)$ & $8.0\left(e^{-}\right), 3.5\left(e^{+}\right)$ & $7.0\left(e^{-}\right), 4.0\left(e^{+}\right)$ & $\mathrm{GeV}$ \\
beam current & $1.8\left(e^{-}\right), 2.7\left(e^{+}\right)$ & $1.2\left(e^{-}\right), 1.6\left(e^{+}\right)$ & $2.6^{*}\left(e^{-}\right), 3.6^{*}\left(e^{+}\right)$ & $\mathrm{A}$ \\
luminosity $\mathcal{L}$ & 1.2 & 2.1 & $80^{*}$ & $10^{34} \mathrm{~cm}^{-2} \mathrm{~s}^{-1}$ \\
int. lumi. $\int \mathcal{L} \mathrm{d} t$ & 0.557 & 1.041 & $50^{*}$ & $\mathrm{ab}^{-1}$ \\
$N_{\text {bunch }}$ & 1732 & 1584 & 2500 & - \\
circumference & 2.2 & 3.016 & 3.016 & $\mathrm{~km}$ \\
horiz. beam size $\sigma_{x}$ & 140 & 170 & 10.7 & $\mathrm{\mu m}$ \\
vert. beam size $\sigma_{y}$ & 3000 & 940 & 62 & $\mathrm{~nm}$ \\
\hline
\end{tabular}

Table 3.1.: Key performance parameters of the $B$-factories PEP-II [18] and KEKB [18] and of the next-generation super- $B$-factory SuperKEKB [19]. Numbers marked with * are target values for peak operation (luminosity, currents) or over the full life-time (integrated luminosity).

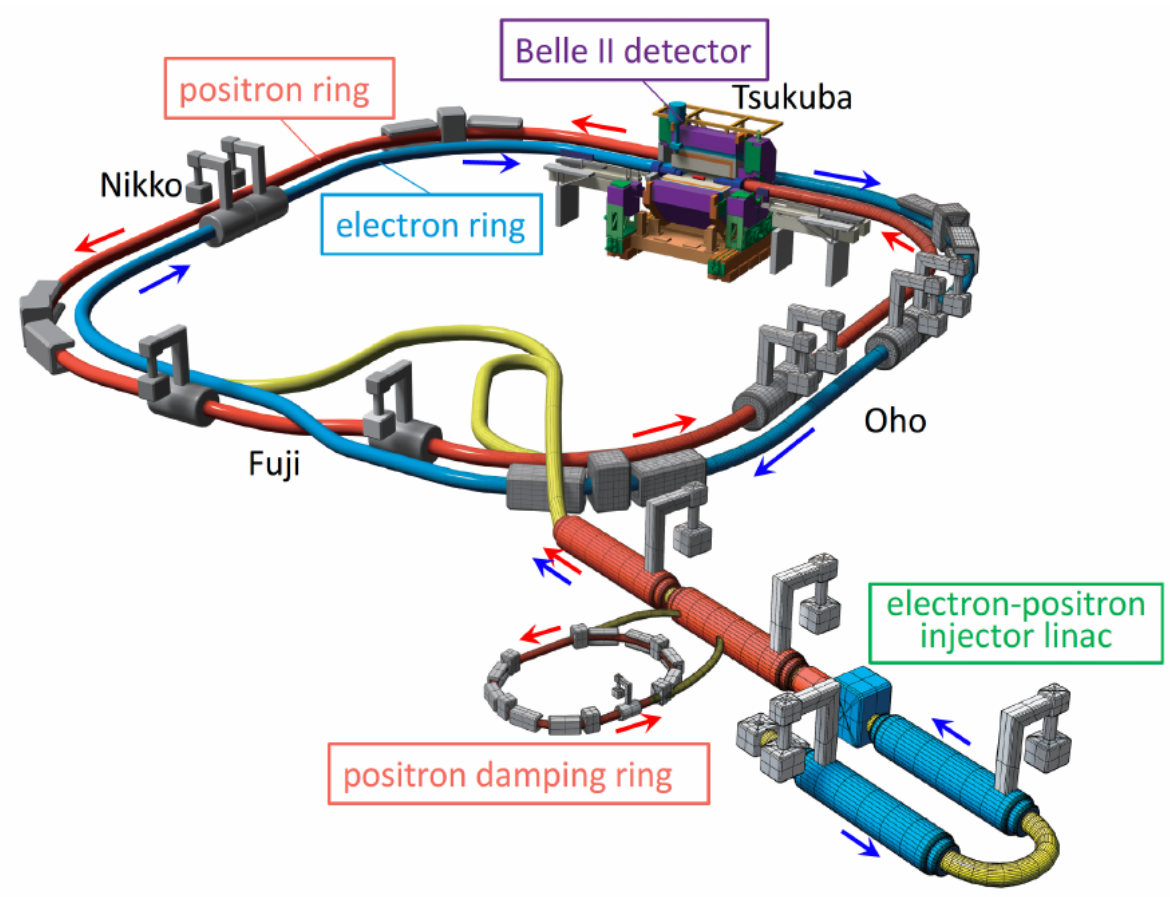

Figure 3.1.: Schematic drawing of SuperKEKB collider components. A linear accelerator (linac) is used for electron and positron production and pre-acceleration. Electrons and positrons are then injected into their respective circular accelerator rings. The location of the collision of the two beams, the interaction point, is surrounded by the Belle II detector. The whole setup is located underground, accessible via four experiment halls (called Fuji, Nikko, Oho and Tsukuba). The Tsukuba hall houses the detector system. Figure is from [19]. 



\section{CHAPTER 4}

\section{The Belle II Experiment - Precision B-physics}

The properties of particles created in $e^{+} e^{-}$-collisions at $B$-factories and their decay products need to be measured by detector systems. Direct and indirect observables like

- momentum, total energy and mass,

- electric charge, and

- lifetime and common production/decay vertices

of individual particles are measured and collision events are reconstructed. From large data sets of such measurements and their statistical analysis, information on

- production rates of individual particle types and

- probabilities for certain decay channels of these particles

can be concluded. Particle collisions created by the KEKB collider from 1999 to 2010 were recorded with a detector system called Belle. Belle II is the successor experiment, reusing some of the mechanical infrastructure of Belle and KEKB, but with major upgrades to the accelerator (SuperKEKB) and of the sub-detectors. The design and components of the Belle II detector are discussed in the following section. A brief and non-complete motivation of the physics program for Belle II is given and the significance of the pixel sub-detector for vertex resolution is emphasised.

The state of knowledge at the beginning of the Belle II data taking period can be summarised as follows:

- The LHC experiments ATLAS [20] and CMS [21] announced the Higgs discovery in 2012 [22,23]. Meanwhile, the Higgs boson and the Spontaneous Symmetry Breaking mechanism giving mass to the elementary particles is established in the SM. Direct constraints on masses of new particles are set and a large fraction of the possible parameter spaces for super-symmetric (SUSY) theories is excluded [24]. 
- LHCb [25] has measured the mixing parameters of the $B_{s}$ meson to be consistent with the SM [26]. Deviations in the ratio of $B \rightarrow K^{(*)}+e^{+}+e^{-}$and $B \rightarrow$ $K^{(*)}+\mu^{+}+\mu^{-}$are observed [27].

- Tensions in the $B \rightarrow \tau+\nu_{\tau}, B \rightarrow D^{*}+\tau+\nu_{\tau}$ and $B \rightarrow K^{*}+\tau+\nu_{\tau}$ decay channels, all involving $\tau$ leptons, are observed among Belle, BaBar and LHCb [26,28]. While experimentally challenging in the subsequent leptonic $\tau$ decays, a unique sensitivity to new physics phenomena is expected in these measurements.

\subsection{Belle II - A multi-purpose particle detector}

Belle II is a multi-purpose $4 \pi$-coverage detector. The attribute "multi-purpose" here recognises the fact that the experiment is built from several sub-detectors, each tailored to measure particular properties of individual particles created in $e^{+} e^{-}$-collisions. The combination of the respective measurements then yields a comprehensive picture of collision events. The detector is built around a well-defined collision region, the interaction point (IP). One design guideline is thus to cover a large fraction of the solid angle around the IP, hence the term " $4 \pi$-coverage". Of course, the detector coverage is limited by the incoming and outgoing beam pipes and structures for mechanical support, cooling and electrical interconnectivity. The Belle II detector has a cylindrical shape that surrounds the $e^{-}$high-energy ring (HER) and the $e^{+}$low-energy ring (LER) where they meet at the common IP beam pipe. The detector has a length of $7.4 \mathrm{~m}$ and a diameter of about $7 \mathrm{~m}$. Inside this volume is a superconducting solenoid of $4.4 \mathrm{~m}$ length and $3.4 \mathrm{~m}$ diameter providing a constant solenoidal magnetic field of $1.5 \mathrm{~T}$ strength oriented parallel to the detector's symmetry axis. A Cartesian right-handed coordinate system is defined with its origin at the nominal IP. The $z$-axis identical to the detector's symmetry axis. The positive $z$-direction is the direction of the boost of the $e^{+} e^{-}$-system (i.e. the direction of the movement of the incoming $\left.e^{-}\right)$. The $x$-axis points outwards of the accelerator ring in radial direction. The $y$-axis, accordingly, points towards the detector top side. The angle $\theta$ is defined in the $y z$-plane with $\theta=0$ defined as parallel to the $z$-axis. The perpendicular angle $\phi$ is defined in the $x y$-plane with $\phi=0$ defined as parallel to the $x$-axis. The detector volume is divided into a forward $\left(17^{\circ} \leq \theta \leq 30^{\circ}\right)$, a barrel $\left(30^{\circ} \leq \theta \leq 125^{\circ}\right)$, and a backward $\left(125^{\circ} \leq \theta \leq 150^{\circ}\right)$ region. The detector design is asymmetric in the forward and backward regions as a consequence of the boost of the collision centre of mass system.

The sub-detectors of Belle II are discussed in the following, going from the IP outwards. The descriptions here are based mainly on target designs and expected performances from R\&D investigations and simulations as given in the Technical Design Report [29] and other sources $[17,30]$. Figure 4.1 shows a technical sketch of the Belle II detector from the top view. 


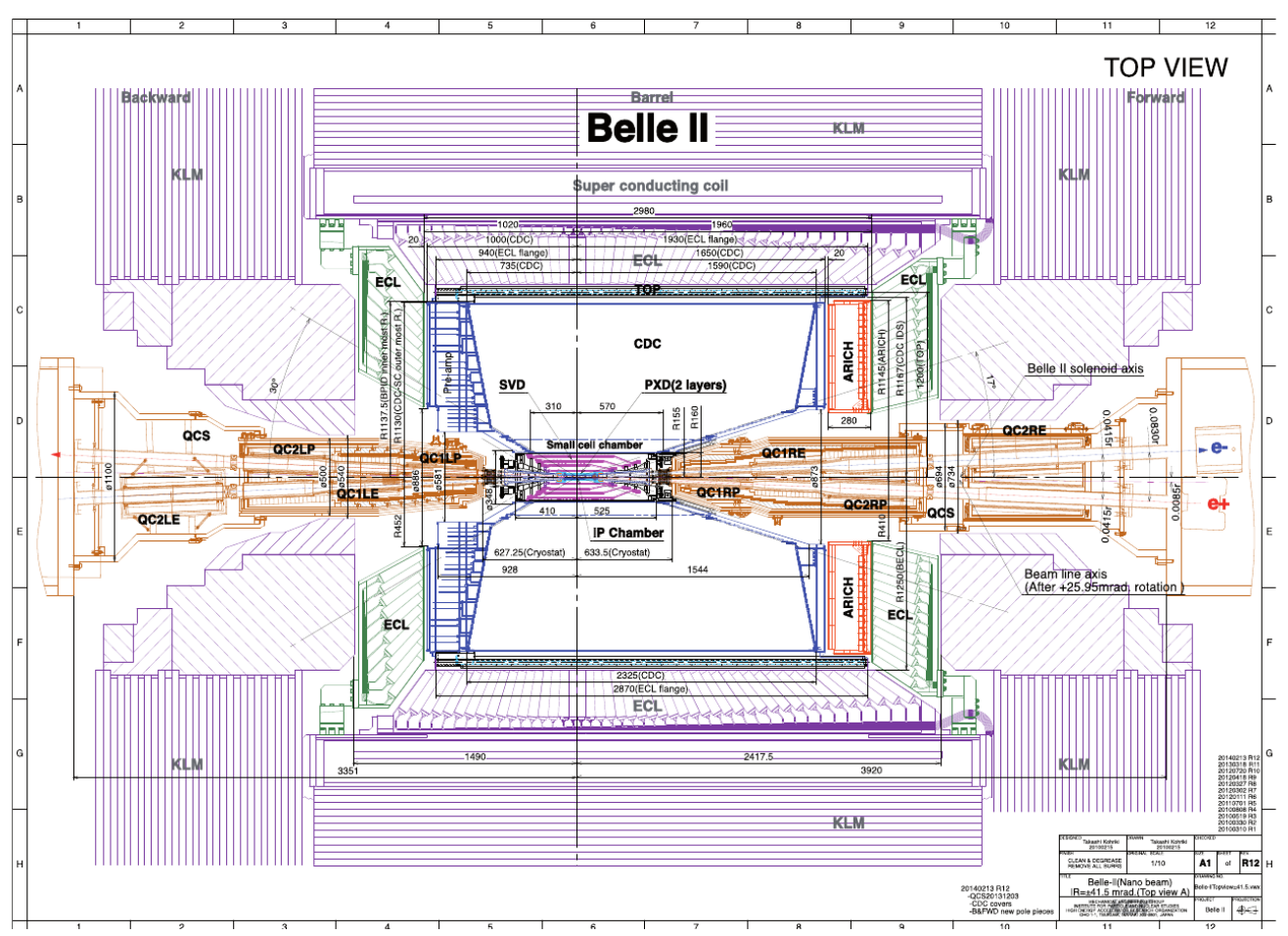

Figure 4.1.: Technical drawing of the Belle II detector as a top view. Figure is from [30].

\subsubsection{Vertex detector (VXD)}

The vertex detector (VXD) is the innermost active detector component ${ }^{1}$ in Belle II. Its purpose is to measure space points of trajectories of charged particles. These space points are ultimately used to reconstruct particle trajectories and thereby measure their decay vertices. Multiple daughter particles stemming from the same decaying mother particle can thus be associated. Due to the magnetic field, charged particles up to momenta of about $30 \mathrm{MeV}$ do not leave the VXD volume and the VXD provides the only source of information for these particles. The VXD is build from silicon semi-conductor based devices. To precisely reconstruct decay vertices, a long "lever arm" on the particle trajectory measurement is beneficial. That means that space points very close to the decay vertex and very far away are needed. Thus, the innermost vertex detectors must be small in volume and located close to the IP. In addition, the disturbance of particle trajectories due to multiple scattering should be minimised. Therefore, the design guideline for the VXD detector comprises small devices with low material budget.

For Belle II, a two-fold approach was chosen. The VXD involves six layers of detector modules for measuring trajectory space points close to the IP. The closer to the IP, the higher is the density and frequency of particles traversing a certain fiducial volume. The

\footnotetext{
${ }^{1}$ The innermost component is the beam pipe made of beryllium and titanium that surrounds the IP and seals the vacuum inside the accelerator rings.
} 


\section{The Belle II Experiment - Precision B-physics}

innermost two layers of the VXD are thus based on a pixel detector (PXD) approach with a high granularity of independent read-out channels. The four outer layers are based on a silicon strip detector (SVD) approach.

The pixel detector (PXD) design builds on the depleted field-effect transistor (DEPFET) principle. This allows for thin and low material budget detector modules with low thermal power dissipation. Since the pixel detector is the main focus in this thesis, a detailed description of the PXD design is given in Chapter 6. For the moment, it should suffice to note, that the two layers of the PXD are built from planar module ladders of $136 \mathrm{~mm}$ (layer 1) and $170 \mathrm{~mm}$ (layer 2) length and $15.7 \mathrm{~mm}$ width arranged in a windmill structure. The layers are located at radii of $14 \mathrm{~mm}$ (layer 1) and $22 \mathrm{~mm}$ (layer 2) around the IP. This is realised by installing the PXD directly onto the beam pipe. The coverage is $17^{\circ} \leq \theta \leq 150^{\circ}$. Each layer of the PXD contributes $0.21 \%$ of a radiation length to the overall material budget.

The silicon strip detector (SVD) $)^{2}$ forms the outer four layers of the VXD. It is likewise built from planar ladders in a windmill arrangement. The layers are located at radii of $38 \mathrm{~mm}, 80 \mathrm{~mm}, 115 \mathrm{~mm}$ and $140 \mathrm{~mm}$. Owing to the reduced expected density of traversing particles, a detector design with lower read-out channel granularity is possible for the outer layers. The SVD is based on double-sided strip detectors (DSSD). These are depleted silicon bulks with $p$-type strip segmented electrodes on one side and orthogonal (or slightly tilted) $n$-type strip segmented electrodes on the other side of the bulk. Strips are connected to read-out channels. Coordinates of traversing particles are measured as the combination of $p$-strip coordinates and $n$-strip coordinates. If the particle density and frequency is high and multiple strips measure signals, the coordinates can not be measured unambiguously. The ladders of layers 4,5 , and 6 are split into a barrel part and a forward part. The barrel parts (and the full layer 3) are built with rectangular modules that are arranged parallel to the $z$-axis. The forward parts are slanted towards the beam pipe and are built from trapezoidal shaped modules. The former have a thickness of the depleted silicon bulk of $320 \mu \mathrm{m}$, the latter have a thickness of $300 \mu \mathrm{m}$. With this geometrical arrangement, a coverage of $17^{\circ} \leq \theta \leq 150^{\circ}$ is established. The read-out chips are mounted on the $n$-side of the sensor and are cooled by evaporative $\mathrm{CO}_{2}$ cooling, with a cooling system shared with the PXD. Each layer of the SVD contributes $0.57 \%$ of a radiation length to the overall material budget. The SVD is mechanically attached to its encapsulating sub-detector. Figure 4.2 sketches the geometry of the VXD sub-detector system.

\subsubsection{Central drift chamber (CDC)}

The central drift chamber (CDC) is a cylindrical multi-wire drift chamber that encloses the VXD. The CDC volume extents from a radius of $160 \mathrm{~mm}$ to a radius of $1130 \mathrm{~mm}$. Its purpose is to measure multiple space points of traversing charged particle trajectories. Charged particles ionise the $\mathrm{He}-\mathrm{C}_{2} \mathrm{H}_{6}$ gas mixture inside the CDC. The created charges drift towards the wires in an electrical field generated by applying potential differences

\footnotetext{
${ }^{2}$ SVD abbreviates "silicon vertex detector" but refers to the silicon strip based VXD layers 3 to 6 only.
} 

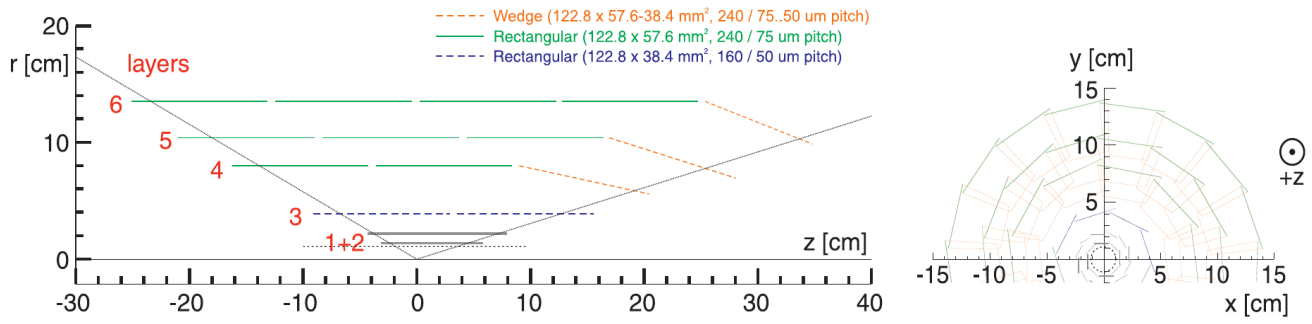

Figure 4.2:: Schematic of the geometry of the individual layers of the VXD sub-detector system. The innermost dashed lines indicates the extent of the beam pipe. The tilted lines indicate the angular coverage of $17^{\circ} \leq \theta \leq 150^{\circ}$. Figure is from [30].

among groups of wires. The charges are collected by a total of 14336 gold plated tungsten wires of $30 \mu \mathrm{m}$ diameter. Each collecting wire is surrounded by eight aluminium field wires forming the drift field. At small radii, the wire density is higher than for large radii, adjusted to the different levels of expected particle density. Most wires are arranged in parallel to the magnetic field. From the bending of the reconstructed trajectory, the transverse momentum of the particle can be determined. Some wires are slightly tilted by about $45 \mathrm{mrad}$ with respect to the magnetic field to also provide a measure for the longitudinal momentum component. Since the energy loss by ionisation per path length in a medium, $\mathrm{d} E / \mathrm{d} x$, depends on the relativistic parameters $\beta \gamma$ of the particle, measuring the particles momentum and the amount of collected charge on the wires ultimately is a measure of the particles rest mass and thus is a method for particle identification. The gas mixture has a radiation length of $640 \mathrm{~m}$, broadly reducing multiple scattering within the $\mathrm{CDC}$ volume. The coverage is $17^{\circ} \leq \theta \leq 150^{\circ}$.

\subsubsection{Particle identification (PID)}

The particle identification system (PID) is designed to facility the differentiation of particle types. In particular, it is arranged to separate $K^{ \pm}$from $\pi^{ \pm}$mesons. The PID system is divided into a barrel region detector, the time of propagation counter (TOP), and a forward region detector, the aerogel ring-imaging Cherenkov detector (ARICH). Both are based on the effect of Cherenkov radiation [31]. A charged particle traversing a dielectric medium at a velocity larger than the phase velocity in that medium emits electromagnetic radiation. Due to continuous emission and interference, an effective light cone is observable, surrounding the traversing particle's trajectory. The opening angle of this cone depends on the particle's relativistic velocity $\beta$ and the (effective) refraction index $n$ of the traversed material. Knowing the particle's momentum from the CDC and measuring the Cherenkov angle gives a handle on the particle's rest mass and is therefore a means of particle identification.

The time propagation counter (TOP) is the barrel part of the PID. The Cherenkov angle is determined by the propagation time of Cherenkov light in quartz bars. The 16 individual TOP detector modules are $2.5 \mathrm{~m} \times 45 \mathrm{~cm}$ and $2 \mathrm{~cm}$ thick rectangular quartz 


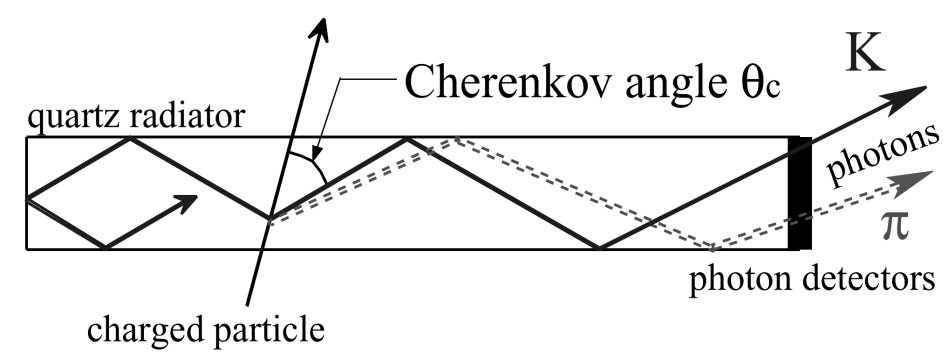

Figure 4.3.: Operation principle of the TOP sub-detector system based on Cherenkov radiation. Figure is from [29].

blocks with a refraction index of $n \approx 1.47$. These modules are arranged at a radius of $1190 \mathrm{~mm}$ around the CDC. Each block has a spherical mirror attached to one of the $45 \mathrm{~cm}$ short sides. The opposite side is covered with photo multiplier tubes (PMTs). A charged particle traversing the quartz radiator causes emission of Cherenkov light. The Cherenkov photons are (partly) confined to the detector volume by total reflection. After typically about 100 reflections and a path length of about $5 \mathrm{~m}$, the photons are collected by the PMTs producing a measurable electrical signal. At identical traversal coordinates, incidence angles and particle momentum, $\pi^{ \pm}$produce a wider light cone compared to $K^{ \pm}$. Cherenkov light by $\pi^{ \pm}$undergoes fewer reflections and produces an earlier PMT signal in comparison to $K^{ \pm}$. Figure 4.3 sketches the operation principle. With a time resolution of about $40 \mathrm{ps}$, the TOP PMT counters are capable of separating $\pi^{ \pm}$signals from $K^{ \pm}$signals. Also the spatial segmentation of the PMTs is utilised. The information on traversal coordinates and incidence angles is provided by the reconstructed trajectory from VXD and CDC measurements. The coverage is $32.5^{\circ} \leq \theta \leq 123^{\circ}$.

The aerogel ring imaging Cherenkov detector (ARICH) is the forward part of the PID. It is arranged perpendicular to the $z$-axis, placed at $z=1670 \mathrm{~mm}$. It spans over the radii $441 \mathrm{~mm}$ to $1115 \mathrm{~mm}$. In contrast to the TOP detectors, the radiator material in ARICH is a special translucent, ultra-low density synthetic gel called aerogel. It is a proximity focusing ring imaging detector, meaning that a thin radiator of about $20 \mathrm{~mm}$ thickness if followed by an expansion gap of about $20 \mathrm{~cm}$ before Cherenkov photons are collected by an array of photo detectors. By using two consecutive layers of aerogel radiators with slightly different refraction indices $\left(n_{1}=1.046\right.$ and $\left.n_{2}=1.056\right)$, overlapping Cherenkov rings are produced. This increases the photon count per ring image while improving the resolution on the measured Cherenkov angle at the same time. The detection is based on segmented hybrid avalanche photo detectors (HAPD). ARICH is expected to provide a $4 \sigma$-level separation of $K^{ \pm}$and $\pi^{ \pm}$in a momentum range of $0.7 \mathrm{GeV} \leq p \leq 4.5 \mathrm{GeV}$. The coverage is $14.78^{\circ} \leq \theta \leq 33.71^{\circ}$.

\subsubsection{Electromagnetic calorimeter (ECL)}

The electromagnetic calorimeter (ECL) measures the energy and angular direction of photons and provides particle identification of electrons/positrons. It is built from 
CsI(Tl) crystals of $6 \mathrm{~cm} \times 6 \mathrm{~cm} \times 30 \mathrm{~cm}$ size. The crystals are arranged in a forward, barrel and backward part. Each crystal is aligned with its long axis pointing to the IP. The forward part consists of 1152 crystals positioned at $z=1.96 \mathrm{~m}$, covering $12.4^{\circ} \leq \theta \leq 31.4^{\circ}$. The barrel part consists of 6624 crystals positioned at a radius of $1.02 \mathrm{~m}$ and pointing outwards, covering $32.2^{\circ} \leq \theta \leq 128.7^{\circ}$. The backward part consists of 960 crystals positioned at $z=-1.02 \mathrm{~m}$, covering $130.7^{\circ} \leq \theta \leq 155.1^{\circ}$. The length of the crystals corresponds to a material budget of 16.1 times the radiation length of the material. Photo diodes at the outward end of the crystals collect scintillation light created by traversing particles. An impinging photon is absorbed producing an $e^{+} e^{-}$pair in the material, which in turn produce photons by annihilation and bremsstrahlung. The total number of photons generated in this electromagnetic shower is the scintillation light that is measured by the photo diodes. For high-energy impinging electrons/positrons ( $220 \mathrm{MeV})$, the shower effect is very similar. The shape of the shower, however, is slightly different due to the fact that the massive particles continuously emit bremsstrahlung along their path until absorbed (or leaving the ECL volume). ECL is sensitive in the energy range $0.02 \mathrm{GeV}$ to $4 \mathrm{GeV}$. Neutral hadrons interact with the ECL material via hadronisation and subsequent electromagnetic showers with overall shower shapes very distinct from photons and electrons/positrons. Overall, the identification of photons is realised by measuring showers without an associated reconstructed particle trajectory from CDC. For electrons/positrons, the momentum of an associated reconstructed trajectory is put in relation to the total shower energy measured by ECL. Photon signatures are separated from neutral hadrons by the shower shape, especially its lateral extent.

\subsection{5. $K_{L}$ and muon detector (KLM)}

The $K_{L}$ and muon detector (KLM) primarily tailored to distinguish between $K_{L}^{0}$ mesons and muons. It is the outermost sub-detector of Belle II located even outside of the solenoid and thus outside of the constant magnetic field. The KLM is composed of alternating layers of $4.7 \mathrm{~cm}$ iron plates (corresponding to 3.9 radiation lengths each) and detector planes. Like the other more outward detectors, KLM is split into a forward part, a barrel part and a backward part. The forward part (covering $20^{\circ} \leq \theta \leq 45^{\circ}$ ), the innermost two layers of the barrel part (covering $45^{\circ} \leq \theta \leq 125^{\circ}$ ) and the backward part (covering $125^{\circ} \leq \theta \leq 155^{\circ}$ ) use strip scintillators read out by PMTs. Due to reduced particle density expected in the outer layers of the barrel part, which starts at a radius of $2019 \mathrm{~mm}$, these are based on glass resistive plate chambers (RPCs) as detector planes, which are cheaper but exhibit longer dead times. Photons and electrons/positrons are absorbed (in ECL or the solenoid) before reaching the KLM. Neutral mesons, notably $K_{L}^{0}$, do not interact with the inner sub-detectors (apart from small interactions within the ECL volume). Reaching KLM, the neutral mesons hadronise in the iron layers and the subsequently produced charged particles are measured in the detector layers. Muons, and charged hadrons that reach the KLM, directly produce signals in the detector layers. Combined with the measurements from VXD and CDC, showers measured in the KLM are checked for associated reconstructed charged particle trajectories. If no associated trajectory is found, the KLM signal is attributed to a $K_{L}^{0}$. If a KLM signal has an 


\section{The Belle II Experiment - Precision B-physics}

associated trajectory, an overall likelihood ratio of muon origin versus charged meson origin (including VXD, CDC and KLM measurements) defines a muon probability. The muon detection efficiency is about $89 \%$ for energies above $1 \mathrm{GeV}$. The $K_{L}^{0}$ detection efficiency is up to $80 \%$ at energies of about $3 \mathrm{GeV}$.

\subsection{Precision measurements and physics program}

Belle II is designed to resolve some of the tensions observed in measurements done at Belle, BaBar and LHCb with SM predictions. Either these tensions prove to be statistical fluctuations only, or Belle II confirms the disagreement with the SM. With a sizeable dataset, Belle II will be more sensitive to extremely rare decays and strongly suppressed flavour processes. In comparison with the $\mathrm{LHCb}$ experiment, that also provides a enormous amount of flavour physics related data, Belle II is sensitive also to "missing energy" events due to its (nearly) $4 \pi$ coverage and the precisely known collision energy. A non-exhaustive list of the Belle II physics program is given here and is based on [30].

$\boldsymbol{C P}$-violation and CKM parameters Although the $C P$-violating nature of the quark sector is well established, there are still questions to be answered. The unitarity triangle is already well constrained by flavour physics measurements of the predecessor $B$-factories and LHC experiments. It is, however, still important to reduce the uncertainties in order to exclude - or reveal - any remaining discrepancies among the CKM parameters (e.g. an actual non-unitarity) or among measurements of the same parameters in different decay channels [32].

Higgs sector An extended Higgs sector could involve one or more charged Higgs bosons $H^{ \pm}$[33]. These could contribute to loop corrections (or even at tree level) of charged weak interactions where the $H^{ \pm}$mediates the interaction similarly to $W^{ \pm}$, but with stronger coupling to more massive particles. The observed tensions in $B \rightarrow D^{(*)} \tau \nu_{\tau}$ decays measured by BaBar [34], Belle [35] and LHCb [36] could be compatible with these models. An extended Higgs sector could also be a source of additional $C P$-violation [37]. Detailed investigation of these tensions are thus crucial and Belle II is well suited to perform these.

Lepton flavour violation Neutrino experiments measure a large contribution in the mixing of $\nu_{\tau}$ neutrinos [38]. Some models for neutrino mass generation mechanisms come with a prediction of a sizeable $\tau \rightarrow \mu \gamma$ transition. Measuring lepton flavour violation (LFV) is therefore an integral part of the Belle II physics program. Especially $\tau$ leptons are to be measured regarding LFV and $C P$-violation contributions, as well as regarding their electrical dipole moment and the value of the anomalous magnetic dipole moment $(g-2)$. 
Dark sector searches A dark sector of new particles coupling to SM particles via new gauge bosons and comprising candidates as constituents of dark matter is discussed. Interactions of these forms would show signatures in Belle II with unbalanced energy in an event. The trigger system is adapted to be sensitive to, for instance, single photon events.

In general, Belle II has several advantages that facilitate the measurements of this physics program. A clean sample of $B \bar{B}$ events is produced in a low background environment - compared to LHC experiments with large pileup. The $B \bar{B}$-system is produced in a coherent state, thus one decay can be used to tag the flavour of the other. There is no "flavour asymmetry" since the colliding particles have the same lepton flavour. The "charged asymmetry", due to the $e^{-}$bringing more energy to the collision than the $e^{+}$, is compensated for by the asymmetric design of the detector. As a result of low charged track multiplicity and excellent vertex resolution (see next section), the reconstruction efficiency for $B$ and $D$ mesons, as well as for $\tau$ decays is high. The boost of the centre of mass system, again in conjunction with good vertex reconstruction capabilities, allows for lifetime measurements via flight lengths. Also time-dependent $C P$-violation measurements are thereby possible. The large angular coverage of the Belle II detector along with the well-known initial state enables to measure "missing energy" decays. These are decays involving for example neutrinos or dark sector particles that do not interact with any of the Belle II sub-detectors and are indirectly inferred by unbalanced — missing - energy. The low background also helps with defining low trigger thresholds for such events.

\subsection{The importance of vertex resolution}

The full reconstruction of the $B \bar{B}$ event decay chains fundamentally rely on the reconstruction and good resolution of particle trajectories and decay vertices. Starting from

- the bare momentum measurement via the bending of charged particle trajectories in the magnetic field,

- over the other sub-detectors and PID relying on momentum and extrapolated intersection coordinates,

- grouping of single reconstructed particles to a common decay vertex,

- measuring lifetimes and time-dependent $C P$-violation,

- exploiting the correlated $B \bar{B}$ state by flavour tagging,

- and finally "measuring" missing energy by balancing energies of reconstructed decay vertices,

to all these a precise vertex reconstruction is crucial. The vertex resolution depends on the intrinsic spatial resolution of the involved tracking detectors - that is the precision 


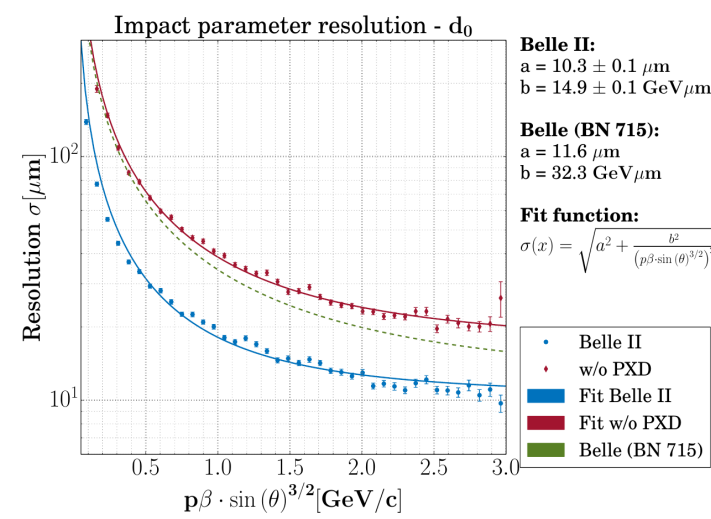

(a) $d_{0}$ resolution

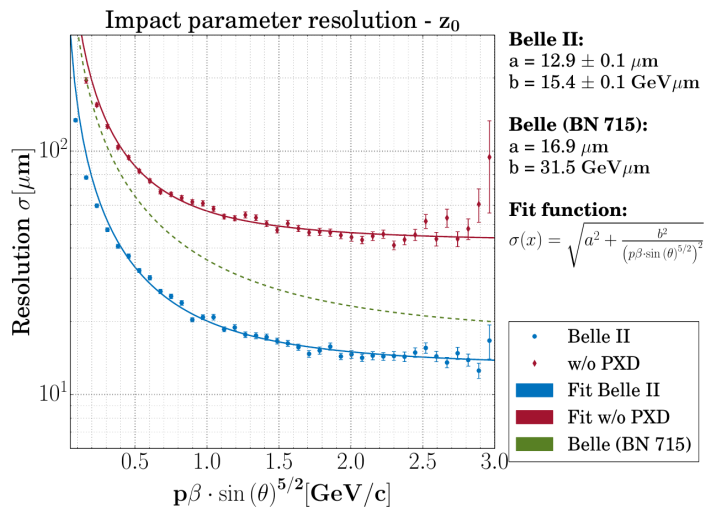

(b) $z_{0}$ resolution

Figure 4.4.: Simulated impact parameter resolution of the Belle II detector in $d_{0}$ (a) and $z_{0}$ (b) compared to the predecessor experiment Belle. Figures are from [17].

at which space points of a trajectory are measured by individual detector planes - and on the uncertainty introduced by multiple scattering. The latter disturbs the trajectory of a charged particle when traversing matter and depends on the particle's momentum. The design goal for the vertex detectors to minimise the material budget originates from this relation. The intrinsic spatial resolution on the other hand has to be optimised under the given boundary conditions. The vertex resolution is quantified as the resolution of the impact parameters $d_{0}$ and $z_{0}$. For a given reconstructed track, the impact parameter $d_{0}$ is defined as the geometrical distance of the point of closest approach of the track projected onto the $x y$ plane to the origin (the nominal IP). The $z$-coordinate of the point of closest approach is the $z_{0}$ impact parameter. The impact parameter resolution has been studied in simulations [17]. An extensive model of the Belle II detector is available for simulating the response of the detector to specific collision events or single particles. A simulation of $\mu^{-}$particles of $0.1 \mathrm{GeV} \leq p \leq 3 \mathrm{GeV}$ momentum in the $1.5 \mathrm{~T}$ magnetic field and covering the full acceptance of the detector has been performed. While the track finding - that is the selection of sub-detector signals belonging to a particle trajectory — is given by the simulation itself, the track fitting is using the algorithm as proposed for real data analysis for Belle II. The estimated impact parameter resolutions, separated in the contributions of intrinsic spatial resolution and multiple scattering degradation, obtained from the simulation are shown in Figure 4.4. The resolution of the predecessor Belle is compared to the Belle II resolution in the scenarios with and without the pixel detector (PXD). The PXD significantly improves the resolution and thus is an important component of the Belle II detector. The obtained intrinsic spatial resolutions are in the order of $10 \mu \mathrm{m}$ for $d_{0}$ and $13 \mu \mathrm{m}$ for $z_{0}$. The measured overall impact parameter resolution in $d_{0}$ has been reported to be $14.1 \pm 0.1 \mu \mathrm{m}$ based on the first available data [39]. Note that the computation of this value follows a different approach than the simulation. For details, the reader is referred to [39]. 
CHAPTER 5

\section{Interaction of Charged Particles with Matter - The Principles of Tracking Detectors}

Today's particle physics experiments are based on very complex detector systems. Usually, like the Belle II experiment, these detectors are a combination of various subdetectors, each with a distinct purpose. Individual properties of particles produced in collisions are measured by these sub-detectors, including their momentum and their electric charge. The deflection of charged particles in a magnetic field, well known from cathode ray experiments [1], provides a mechanism for measuring these properties for charged particles. In a constant and uniform magnetic field $\vec{B}$ of strength $B$ (in units of T), a particle with electric charge $q$ (in units of elementary charge $e$ ) and momentum $p$ (in units of $\mathrm{GeV}$ ) is forced on a helix shaped trajectory with radius $R$ (in units of $\mathrm{m}$ ) and angle $\lambda$ according to

$$
p \cos \lambda=0.3 \cdot q B R .
$$

The quantity $p / q$ can thus be measured by determining the bending radius $R$ in a known magnetic field $B$. The task is thus to infer the bending radius $R$ by measuring the particles trajectory.

In the language of high-energy physics particle detectors, charged particle trajectories are called tracks. Detector systems that are sensitive to traversing charged particles are used as tracking detectors. The basic idea is to measure individual space points ${ }^{1}$ of each particle track. Several measured space points are combined to form a model of the full particle track (track fitting). Ideally, the space points are measured without disturbing the particle track. In reality, of course, particles traversing a detector loose a fraction of their energy (ionisation) and can be deflected (multiple scattering). In fact, this must be the case in order to produce a measurable signal in the detector. The task

\footnotetext{
${ }^{1}$ Also timing information can be crucial in case of a high particle rate in order to resolve signal ambiguities.
} 
when designing tracking detectors is to minimise the disturbance of the particle tracks while providing a good and efficient measurement of particle passages.

This chapter discusses the principles of silicon semiconductor based tracking detectors. A general understanding of energy loss of charged particles in matter is necessary to understand the charge generation and collection in silicon semiconductor devices. Spatial information can be obtained from the processed detector signals.

\subsection{Energy loss in matter}

Electrically charged particles traversing matter interact with the atoms and (shell-) electrons of the material. For heavy charged particles, that are all particles with a mass larger than the electron mass, the results of the interaction are ionisation or (atomic) excitations. The average energy loss per path length $\left\langle\frac{\mathrm{d} E}{\mathrm{~d} x}\right\rangle$ as a function of the relativistic parameters of the particle $\beta \gamma$ is given by the Bethe-Bloch equation (Eq. 5.2, refer to [4] for an explanation of the various quantities). It describes the average energy loss including rare events with large energy loss, which can cause secondary, high-energetic knock-on electrons called $\delta$-electrons.

$$
-\left\langle\frac{\mathrm{d} E}{\mathrm{~d} x}\right\rangle=K z^{2} \frac{Z}{A} \frac{1}{\beta^{2}}\left[\frac{1}{2} \ln \frac{2 m_{e} c^{2} \beta^{2} \gamma^{2} W_{\max }}{I^{2}}-\beta^{2}-\frac{\delta(\beta \gamma)}{2}\right]
$$

Tracking detectors are often built of thin (several $100 \mu \mathrm{m}$ ) layers of sensitive materials. For thin layers, the distribution of energy losses is not Gaussian [40] and thus the mean energy loss is not a useful quantity. Instead, the asymmetric energy loss probability distribution is best described by a Landau distribution. It features a most probable value (MPV) peak and a long tail towards high energy losses. Figure 5.1 shows an example of the Landau-shaped energy loss for $\pi^{ \pm}$mesons of energy $500 \mathrm{MeV}$ in silicon material of different thickness. Especially for very thin layers of about $80 \mu \mathrm{m}$, the distribution is highly skewed.

Electrons and positrons undergo additional interactions contributing to the energy loss. These are mostly bremsstrahlung and to a smaller extend Møller-scattering for electrons and annihilation and Bhabha-scattering for positrons. Especially for highenergetic electrons/positrons, bremsstrahlung is the dominant interaction with an energy loss proportional to the particles energy (Eq. 5.3). The proportionality factor is a material dependent quantity called radiation length $X_{0}$.

$$
-\left\langle\frac{\mathrm{d} E}{\mathrm{~d} x}\right\rangle_{\text {brems. }}=\frac{x}{X_{0}}
$$

For silicon, the value of the radiation length is $X_{0}^{\mathrm{Si}}=9.36 \mathrm{~cm}$ [4]. Figure 5.2 indicates the energy-dependent contributions of the various interactions for electrons/positrons. Besides the energy loss, also the deflection of the particle's direction of movement in matter is of importance. Charged particles undergo elastic scattering off the Coulomb potential of the materials nuclei. The multiple scattering net effect on the particles 


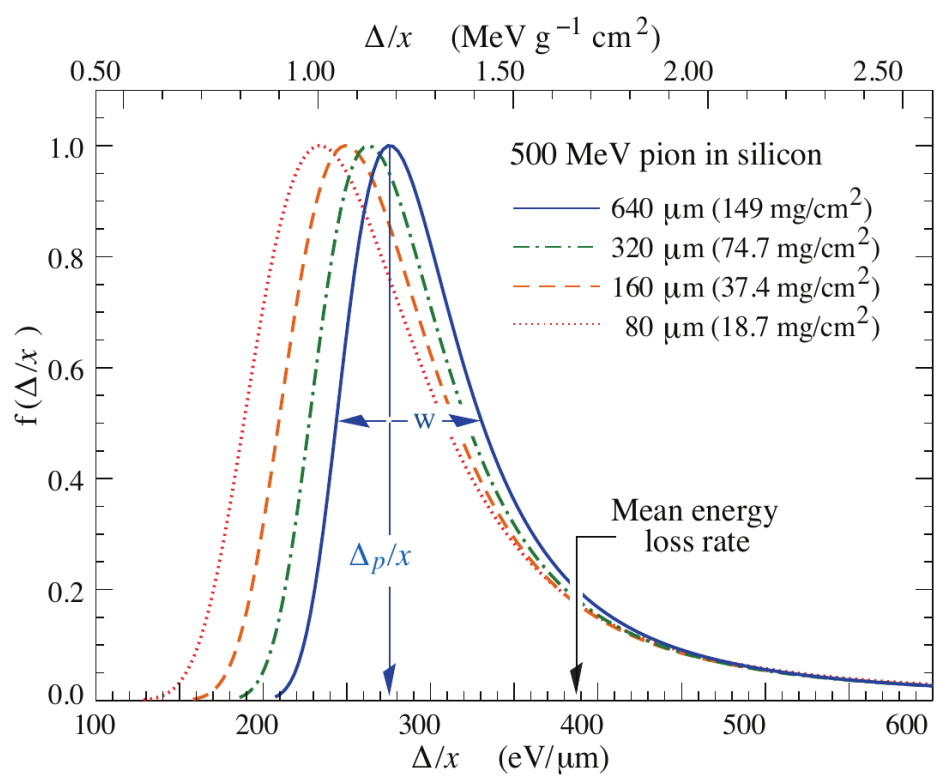

Figure 5.1.: Landau-shaped energy loss of charged particles $\left(\pi^{ \pm}\right)$in thin layers of silicon. The energy loss is here divided by length and normalised to unity at the MPV $\Delta_{p} / x$ for comparability. Figure is from [4].

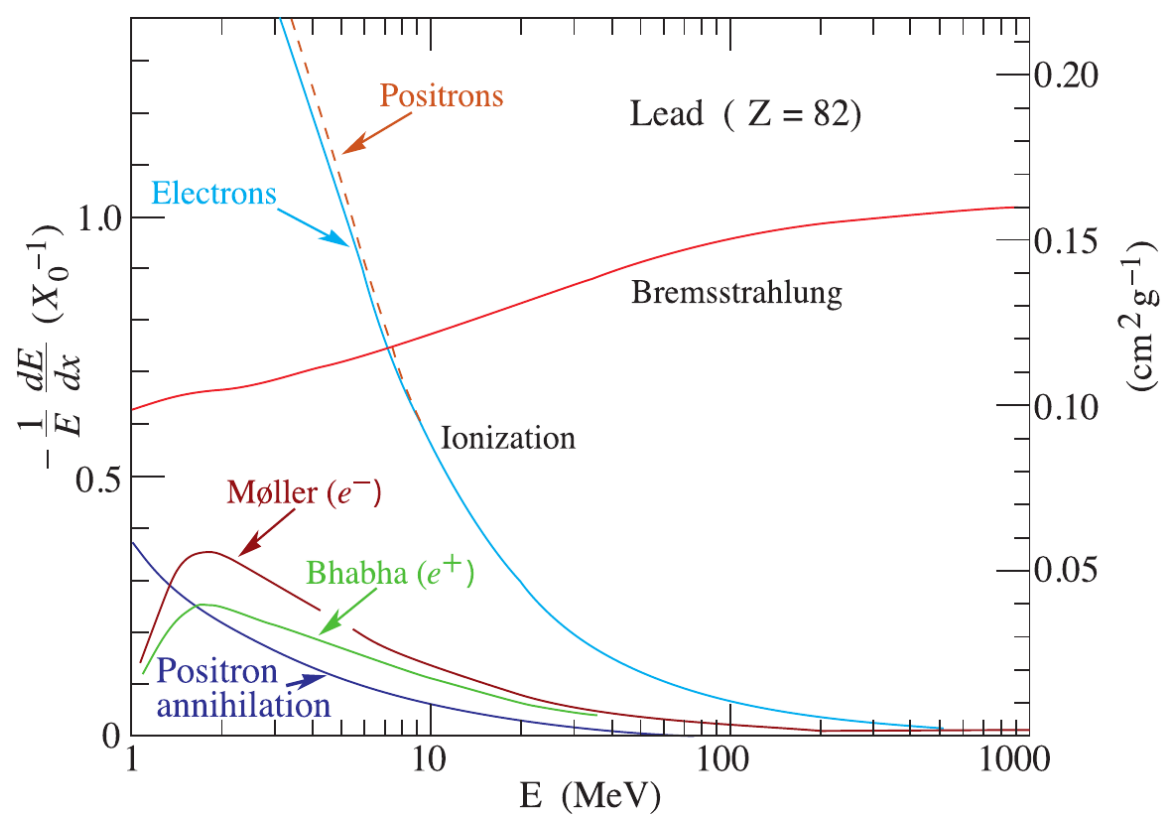

Figure 5.2.: Normalised energy loss per radiation length of interactions of electrons and positrons of different energies in lead $(\mathrm{Pb})$. Figure is from [4]. 


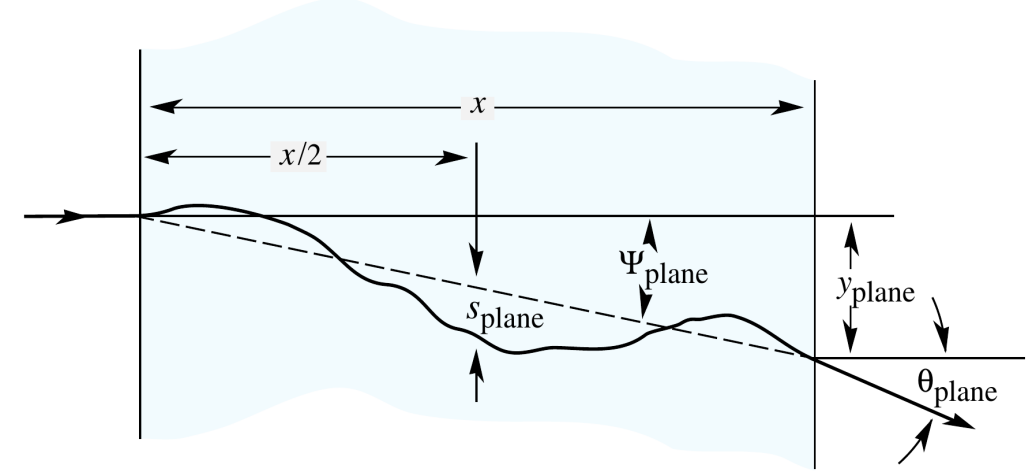

Figure 5.3.: Sketch of the influence of multiple scattering on the trajectory of a particle traversing matter. Figure is from [4].

trajectory when traversing a certain material thickness is a statistical process. Figure 5.3 sketches the effect of multiple scatering. For very thin material layers, the offset $y$ is negligible. The distribution of net scattering angles $\theta$ can be described by a Gaussian distribution with a width $\theta^{\text {rms }}$ as a function of the thickness $x$ given in Eq. 5.4 [4].

$$
\theta^{\mathrm{rms}}=\frac{13.6 \mathrm{MeV}}{\beta p c} z \sqrt{\frac{x}{X_{0}}}\left(1+0.038 \ln \frac{x}{X_{0}}\right)
$$

This model is valid for the central Gaussian core of the distributions, while it fails to describe single large angle scattering [41].

\subsection{Silicon semiconductor detectors for particle tracking}

Silicon semiconductor based tracking detectors are a well established technology in high energy physics experiments. Compared to gaseous detectors, semiconductor detectors usually exhibit better time and spatial resolution and require a smaller detector volume due to the higher density of the solid material. Many variants of semiconductor based tracking detectors exist and are being developed, each tailored to specific needs and optimising specific performance parameters. This chapter introduces the basic mechanisms of charge generation and collection in silicon semiconductor devices, that are common to most of the design variants. A brief overview of the concepts of semiconductors is given.

\subsubsection{Charge generation and collection}

A charged particle traversing matter deposits a certain amount of energy $E_{\text {dep }}$ in the material. Due to ionisation, free electrons and holes (missing bound electron states) are created. For each electron-hole pair, a certain energy $\Delta E_{e h}$ is needed. The number $N$ of electron-hole pairs generated by the traversing charged particle is given as the ratio 
of the two (Eq. 5.5).

$$
N=\frac{E_{\text {dep }}}{\Delta E_{e h}}
$$

In silicon, the average required energy $\Delta E_{e h}$ is about $3.6 \mathrm{eV}$ [4] at room temperature. Both electrons $e$ and holes $h$ are mobile. Their mobility is governed by statistical movement and diffusion due to gradients in concentrations. Eventually, free electrons and holes will recombine. In the presence of an electric field, the charge carriers experience an additional drift movement separating electrons and holes. In the presence of a magnetic field, also the Lorentz force plays a role. It changes the drift direction of the charge carriers relative to the electric field under a Lorentz angle $\theta_{L}$. By separating and collecting the charge carriers, the total deposited charge $N e^{(-)}$, or $N h^{(+)}$respectively, can be measured. Since for field strengths up to about $1 \mathrm{kV} \mathrm{cm}^{-1}$ the mobility for electrons is about three times higher compared to holes, the collection of electrons is usually preferred for faster signal rise times. Ultimately, this procedure provides a measurement of the deposited energy.

\subsubsection{Silicon semiconductor sensors}

Semiconductors are materials with an electrical conductivity in between the regime of conductors and insulators. While the electrical resistivity increases with temperature for conductors, it decreases for semiconductors. Silicon $(\mathrm{Si})$ is a well established and widely used semiconductor material. The concentration of free charge carriers in silicon can be influenced by introducing doping materials which add stationary (non-mobile) impurities. Additional donor (n-doped) materials (e.g. phosphorus) shift the free charge concentration balance towards electrons, acceptor (p-doped) materials (e.g. boron) shift the charge concentration balance towards holes. When p-doped and n-doped silicon material are brought together, a pn-junction is created. The majority charge carriers of both sides recombine yielding a volume depleted of free charge carriers with only stationary space charges (depletion region). The respective space charges cause a temperature and doping concentration dependent electric potential called built-in voltage $V_{\mathrm{bi}}$. Applying an additional external potential $V_{\text {bias }}$ in the same direction (reverse bias) increases the depletion zone. The depth $d$ of the depletion region is given by Eq. 5.6 where $\epsilon_{0}$ and $\epsilon_{\mathrm{Si}}$ are the dielectric constants $\left(\epsilon_{0} \epsilon_{\mathrm{Si}}=\epsilon \approx 1 \mathrm{pF} / \mathrm{cm}\right), e$ is the elementary charge and $N_{A}$ and $N_{D}$ are the respective acceptor and donor concentrations and $N_{\text {eff }}$ is the effective doping concentration.

$$
d=\sqrt{\frac{2 \epsilon_{0} \epsilon_{\mathrm{Si}}}{e}\left(\frac{1}{N_{A}}+\frac{1}{N_{D}}\right)\left(V_{\mathrm{bias}}+V_{\mathrm{bi}}\right)}=\sqrt{\frac{2 \epsilon\left(V_{\mathrm{bias}}+V_{\mathrm{bi}}\right)}{e N_{\mathrm{eff}}}}
$$

Charges generated in this depletion region are collected by the biasing electrodes and form the measurable signal current or integrated charge. Thermally generated charges also contribute to the measured charge or current (leakage current). Since these do not belong to the actual particle signal, this contribution is considered noise degrading the measurement. The noise contribution is statistical and not constant. A sensor is 
characterised in terms of noise via the signal-to-noise ratio (SNR). Another component degrading the measurement is charge trapping. Before reaching the collecting electrode, charge carries can be trapped for example in impurities in the silicon crystal lattice. The density of impurities and crystal defects also changes the doping concentrations and thus the required depletion potential increases with radiation.

\subsection{Spatial information in pixel detectors}

By segmentation of the collecting electrodes, spatial information on a charged particle passage can be obtained. This segmentation can be one-dimensional (strips), or twodimensional (pixels). Strip sensor devices have less read-out complexity and can often be built cheaper compared to pixel sensor devices. However, strip sensors exhibit limitations in the resolution of the spatial information and, especially at high particle rates, suffer from ambiguities in combining one dimensional strip information to two dimensional space points. Pixel sensor devices are in the focus of this thesis.

In a pixel sensor, the sensitive area is segmented in a (usually) regular two dimensional grid of cells called pixel matrix. The distance between neighbouring pixels is the pixel pitch $P$, which can be different in the two directions. It has the most significant influence on the sensors spatial resolution. The spatial resolution $\sigma$ is the precision at which spatial information can be provided by a sensor. For a purely binary read-out, that means each pixel provides a binary signal (hit or no hit) depending on the collected charge in relation to a defined threshold value and a traversing particle causing only a single pixel hit, the spatial resolution is given by Eq. 5.7 [42].

$$
\sigma=\frac{P}{\sqrt{12}} \approx 0.29 P
$$

Due to the statistical distribution of the generated charges, the read-out thresholds are usually set as low as possible. There is a trade-off between not missing traversing particles (efficiency) and the number of firing pixels due to electrical noise (noise occupancy). When a traversing particle is hitting the corners or edges of a pixel, or when its trajectory is highly inclined, it is possible for the generated charge to be shared by multiple neighbouring cells (charge sharing). While the individual pixel charge signals are compared to the respective pixels thresholds, all neighbouring firing cells are summed up to a cluster with an overall cluster charge that is the sum of the associated pixels. In the presence of charge sharing, the position of the particle passage can be localised better yielding a smaller spatial resolution. This approach can be improved further by considering the relative weights of the charges collected by the individual pixels in a cluster, at the cost of a more complex read-out system. Algorithms employing charge sharing and charge weighting for improved spatial resolution are discussed in Chapter 8 in the context of beam test measurements. 
CHAPTER 6

\section{A DEPFET-based Pixel Detector for Belle II}

Since the 1980s, silicon semiconductor devices have a successful history in high-energy physics experiments. Existing designs have been continuously improved and new sensor concepts have been developed and employed. The classical hybrid pixel detector design consists of a depleted planar diode with segmented collecting electrodes and a read-out chip bump-bonded on top with a one-to-one connection of read-out electrodes to readout channels [42]. Limitations of this design are the radiation-hardness, read-out speed and the overall material budget. Approaches for mitigating these disadvantages are for example three dimensional silicon sensors, where the electrodes are not planar but vertically penetrate the diode material [42]. A more fundamentally different concept is a monolithic integration of the read-out electronics into the silicon material of the sensor itself. This spares the delicate bump-bonding procedure, but couples the design and production of the sensor and read-out electronics in a more complex processing of the silicon wafers. Monolithic designs are for example based on complementary metal-oxidesemiconductor (CMOS) components. Another semi-monolithic design approach uses amplification transistors on a depleted high-resistivity silicon bulk called depleted (pchannel) field-effect transistors (DEPFET). The DEPFET principle is discussed in this chapter. A DEPFET based tracking detector has been designed for the Belle II experiment and is now being successfully operated. The details of the design of Belle II DEPFET pixel detector modules ${ }^{1}$ is described here. A brief overview of the performance of the currently installed and running pixel detector is given.

\footnotetext{
${ }^{1}$ The term "module" is used to describe the smallest functional unit the pixel detector is built of.
} 


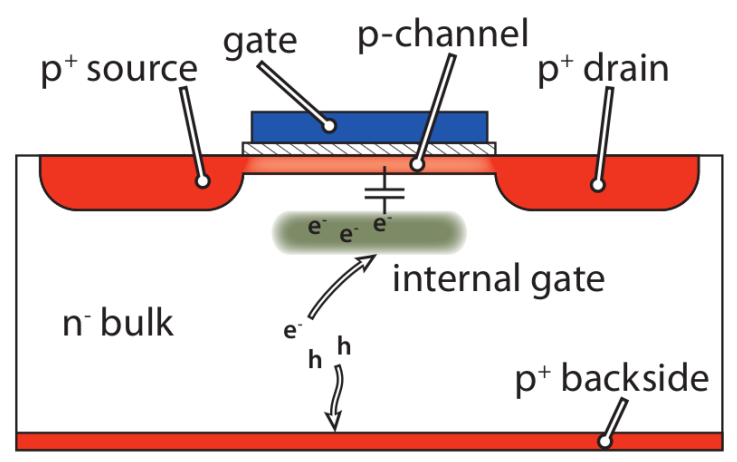

Figure 6.1.: Schematic cross-section of a DEPFET cell. Figure is from [44].

\subsection{The DEPFET operation principle}

A detector concept based on amplification transistors on a depleted silicon bulk was first proposed by Kemmer and Lutz in 1987 [43]. Today, this concept is known as a depleted (p-channel) field-effect transistor (DEPFET). Figure 6.1 shows a cross-section of a single DEPFET cell. The bulk material is lightly $\mathrm{n}$-doped (indicated as $\mathrm{n}^{-}$) highresistivity silicon material with a highly p-doped (indicated as $\mathrm{p}^{+}$) backside contact. In contrast to the conventional hybrid detector design, the read-out electrode is not simply a segmented p-doped contact. Instead, metal-oxide-semiconductor field-effect transistor (MOSFET) structures are integrated on top of the bulk material. The pchannel MOSFET is built from two highly p-doped electrodes forming the drain and the source contact of the transistor. In between, separated by an insulating silicon oxide layer, a negative potential contact forms the transistor gate. When the bulk is depleted and a charged particle traverses the DEPFET cell, electrons and holes are generated and separated. The holes drift towards the backside contact and are removed. The electrons drift towards an additional highly n-doped region below the DEPFET's gate. This internal gate collects the charge (electrons) generated by the traversing particle. This negative charge acts as an additional negative potential (indicated as a capacitive coupling) opening the transistor channel and thus modifying the transistor's source-drain current $I_{S D}$. The change in the transistor's source-drain current thus is an indirect measurement, with an integrated amplification, of the energy deposited by the traversing particle. The amplification factor $g_{q}$ is expressed as the change in $I_{S D}$ per accumulated charge $Q_{\text {internal }}$ in the internal gate (Eq. 6.1).

$$
g_{q}=\frac{\partial I_{S D}}{\partial Q_{\text {internal }}} \approx \frac{\mu}{L^{2}}\left(V_{\text {gate }}-V_{\text {threshold }}\right)
$$

The amplification is approximately linear and depends on the length $L$ of the gate of the MOSFET structure, the charge carrier mobility $\mu$, and the applied gate potential $V_{\text {gate }}$ in relation to the transistor threshold voltage $V_{\text {threshold }}$.

The depletion of the bulk is done via sidewards depletion. Figure 6.1 demonstrates that the DEPFET cell with the n-doped bulk and both p-doped backside and source 


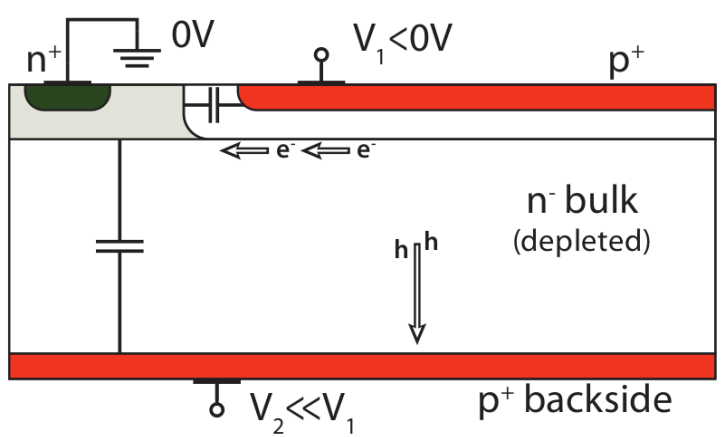

Figure 6.2.: Sketch of the process of sidewards depletion. Figure is from [44].

and drain electrodes essentially forms a double diode structure. By placing an additional n-contact - usually close to the edges of the sensor volume for easier contacting - and applying appropriate biasing potential, the depletion of the full bulk volume can be achieved. Figure 6.2 illustrates this sidewards depletion method. The highly n-doped depletion contact is kept at ground potential. Negative voltages $V_{1}$ and $V_{2}$ are applied to both p-contact sides and two depletion regions grow inside the bulk. $V_{1}$ and $V_{2}$ are chosen such that the full bulk volume is depleted. For electrons, the potential minimum is at the border where the two depletion regions touch. Choosing asymmetric voltages $V_{1}$ and $V_{2}$ allows for vertically moving the potential minimum. For DEPFET cells, it is most convenient to shift the potential minimum close to the MOSFET structures in order to help guiding the electrons drift into the internal gate. In the DEPFET design, the topside p-contacts are the MOSFET source and drain p-implants, which in turn define $V_{1}$.

Since the electrons are collected in the internal gate, the pixel can be read out multiple times (double-sampling). However, this also means that the collected charge needs to be actively removed in order for the cell to be sensitive to new particle passages. A dedicated clear mechanism is implemented via another MOSFET structure as depicted in Figure 6.3. Shown is a cross-section perpendicular to the sketch in Figure 6.1, looking directly onto the p-channel. The internal gate acts as the source for this clear FET. A dedicated highly n-doped contact, shielded by a deep (i.e. highly p-doped) p-well for repelling electrons during normal charge collection, is the drain of the clear FET. The gate of the clear FET (cleargate) can be used to switch the transistor and create a conducting channel between the internal gate and the clear contact removing the internal charges.

Compared to the conventional hybrid detector design, a DEPFET pixel detector requires a collection of dedicated potentials that need to be fine tuned with respect to each other for a proper functioning of all sensor cells. The depletion of the silicon bulk is steered by the depletion (high-)voltage $V_{\mathrm{HV}}$ and the drain and source voltages $V_{\text {drain }}$ and $V_{\text {source }}$ and requires the bulk potential $V_{\text {bulk }}$ to be higher than all of the other voltages. The read-out FET of the cell must be switched between an off-state (charge collection) with potential $V_{\text {gate-off }}$ and an on-state (charge sampling) with potential $V_{\text {gate-on }}$. 


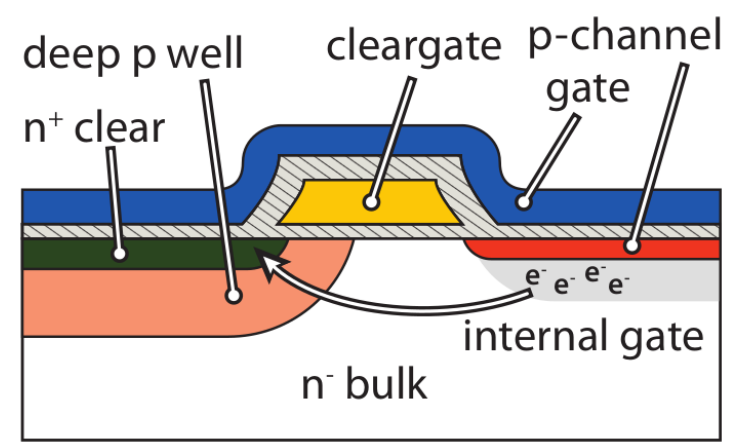

Figure 6.3.: Sketch of the clear FET structure which is implemented to remove charges from the internal gate once the DEPFET cell has been read-out. Figure is from [44].

Similarly, the clear FET requires an appropriate clear-drain potential $V_{\text {clear }}$ and two potentials for switching the clear transistor $V_{\text {clear-off }}$ and $V_{\text {clear-on }}$.

The main advantage of a DEPFET based pixel detector is its low-noise performance [42, 45]. A very low input capacitance in the order of $\mathrm{fF}$ together with the intrinsic amplification mechanism due to the charge-to-current conversion make this possible. Low-noise operation make DEPFETs suitable for low-energy X-ray astronomy [46]. In particle physics detectors, the low-noise feature enables to build very thin detectors ${ }^{2}$. DEPFET sensors with a thickness of only $50 \mu \mathrm{m}$ have been built [47]. Due to the switchable readout of the DEPFET cells, very low power consumption is possible, reducing the need for complex cooling structures with a large material budget. Thin detectors and overall low material budget are preferred in particle physics experiments for reducing the influence of multiple scattering on particle trajectories.

\subsection{Detector modules for Belle II}

The pixel detector for the Belle II experiment is based on the DEPFET principle. The detector is built from several units that can in principle be operated independently. These units are called modules. Each module is a single piece of silicon with a sensitive area (sensor), which is a grid of DEPFET pixels on a depleted silicon bulk. Around the sensor area, electronics is present providing analogue and digital data read-out. An interconnection to the module provides all biasing and operation voltages and links the digital communication between the module and the back-end system. The specific design of these modules for Belle II is described in the following, going from the small scale pixel layout to the full module scale and finally to the full pixel detector scale.

\footnotetext{
${ }^{2}$ Thinning of the sensitive volume reduces the deposited charge while the intrinsic noise is unchanged. Requiring a comfortable signal-to-noise ration (SNR) thus gives a direct constraint on the sensor thickness.
} 


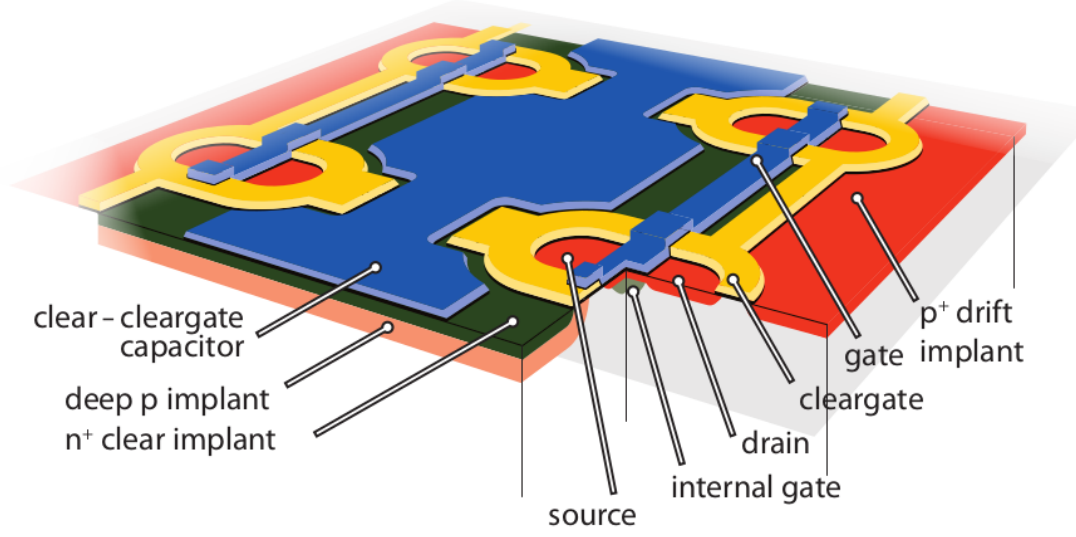

Figure 6.4.: Sketch of the four-fold pixel design implemented in the Belle II pixel detector modules. Figure is from [44].

\subsubsection{Pixel matrix design and biasing}

The DEPFET pixels for the Belle II pixel detector use a gate length of $L=5 \mu \mathrm{m}$. This results in an expected amplification factor $g_{q}$ of $400 \mathrm{pA} /$ electron to $600 \mathrm{pA} /$ electron [29]. The insulating oxide layers of all FETs are kept thin to reduce effects of ionising irradiation damage [48]. A drain read-out is implemented, where the source and drain potentials of the DEPFET are kept constant and the source-drain current $I_{S D}$ is measured $^{3}$. In contrast to the DEPFET gate, which is switched directly, the charge clearing FET is designed with a capacitive coupling of the cleargate to the clear potential $V_{\text {clear }}$ itself. A large positive potential pulse applied to $V_{\text {clear }}$ both opens the cleargate (through the capacitive coupling) and makes the clear electrode more attractive to the electrons in the internal gate. This way, the time required for clearing can be kept very short.

The pixel detector modules implement a four-fold pixel design. Neighbouring pixels in a $2 \times 2$ grid share electrode contacts as sketched in Figure 6.4. The DEPFET gate potentials are connected, such that the pixels are simultaneously switched for read-out. The source and clear contacts are shared. The DEPFET drains are contacted individually, thus each pixel's source-drain current is sampled independently. The cleargate radially surrounds each DEPFET and couples on one side to the common clear-cleargate capacitor. Additional highly $\mathrm{p}$-doped $\left(\mathrm{p}^{+}\right)$drift implants opposite to the shared structures modify the drift paths of electrons for a faster collection in the internal gate. A negative drift potential $V_{\text {drift }}$ is applied. The double-cell pattern is repeated in the direction of the shared clear structures for a total of 250 pixels. The distance between two pixels, called the pixel pitch, in this dimension is $50 \mu \mathrm{m}$. In the perpendicular dimension, an adjacent double-row of pixels is placed. This is repeated for a total of 768 pixels. The distance between two double-rows varies, pixel pitches of $55 \mu \mathrm{m}, 60 \mu \mathrm{m}, 70 \mu \mathrm{m}$ and $85 \mu \mathrm{m}$ are used in that dimension. In this fashion, the $250 \times 768$ grid of the DEPFET

\footnotetext{
${ }^{3}$ An alternative is the source follower mode, where the change in the source potential is measured. This approach, however, yields too slow signal rise times for the given application [49].
} 


\section{A DEPFET-based Pixel Detector for Belle II}

\begin{tabular}{lrl}
\hline voltage & value $[\mathrm{V}]$ & comment \\
\hline$V_{\mathrm{HV}}$ & -60 & depletion high-voltage (punch-through) \\
$V_{\text {bulk }}$ & 10 & n-doped depletion contact \\
$V_{\text {source }}$ & 6 & DEPFET source potential \\
$V_{\text {drain }}$ & 1 & DEPFET drain potential \\
$V_{\text {gate-on }}$ & -2 & DEPFET gate potential during charge sampling \\
$V_{\text {gate-off }}$ & 5 & DEPFET gate potential during charge collection \\
$V_{\text {clear-on }}$ & 19 & clear pulse potential for charge removal \\
$V_{\text {clear-off }}$ & 5 & shielded clear contact \\
$V_{\text {cleargate }}$ & 0 & clear FET gate potential \\
$V_{\text {drift }}$ & -5 & supporting drift field \\
$V_{\text {guard }}$ & -5 & guard ring \\
\hline
\end{tabular}

Table 6.1.: Standard DEPFET biasing and read-out potentials used in the Belle II pixel detector.

pixel matrix is formed. In order to prevent electrons from outside the pixel matrix from entering the sensitive volume, a highly n-doped $\left(\mathrm{n}^{+}\right)$ring surrounds the matrix. This guard ring is biased with a negative potential $V_{\text {guard }}$.

The bulk thickness is only $75 \mu \mathrm{m}$, resulting in a very low material budget of the sensitive volume. Depletion of the bulk is realised via a punch-through mechanism. A backside contact for applying the depletion voltage $V_{\mathrm{HV}}$ complicates the processing of the sensor. Instead, having all biasing contacts on the front side is preferred. A dedicated highly p-doped contact on the front side is used to apply $V_{\mathrm{HV}}$, which produces a localised depletion region growing from the front to the back. Once the $\mathrm{p}^{+}$backside is reached, with increasingly negative $V_{\mathrm{HV}}$, the full bulk eventually becomes depleted - the depletion voltages punches through. For a $75 \mu \mathrm{m}$ thick bulk, a plain depletion voltage of about $-35 \mathrm{~V}$ would be required. Due to the punch-through, twice as much is effectively needed at the punch-through contact: $V_{\mathrm{HV}} \approx-70 \mathrm{~V}$. Table 6.1 lists the standard values for the various potentials used in this design. Most of these voltages have to be fine-tuned for each individual pixel detector module to yield optimal performance.

\subsubsection{Front-end read-out}

The sampling of the drain currents of the DEPFET pixel matrix follows a rolling-shutter mode: Rows of pixels are consecutively read out. The rolling-shutter read-out holds some significant advantages. Electronics processing the drain currents and switching the gate voltages can be placed outside of the active region. In particular, this avoids having a read-out chip on top of the sensor as realised in a hybrid pixel design. This significantly reduces the material budget a traversing particle crosses. Also, thermal power dissipation by transistor currents is greatly reduced since only one row of pixels is active at a time. 
In the present design, the transistor current of a single DEPFET pixel is in the order of $100 \mu \mathrm{A}$. The potential difference between source and drain is about $5 \mathrm{~V}$, yielding a thermal dissipation of roughly $500 \mu \mathrm{W} /$ pixel. For a row of 250 pixels, this sums up to $125 \mathrm{~mW}$.

The drawback of a rolling-shutter mode is an overall read-out time $T_{\text {ro }}$ that depends on the dimension of the pixel matrix. Reading out all pixels of the matrix (a full frame) takes $T_{\text {ro }}=N \times t_{\text {row }}$ where $N$ is the length of the pixel matrix and $t_{\text {row }}$ the read-out time for a single row. The Belle II pixel detector design is optimised for a frame time of $T_{\text {ro }}=20 \mu \mathrm{s}$ [29]. The drain current settling and digitisation sets a lower limit of about $t_{\text {row }}>100 \mathrm{~ns}$ [50]. A single-sampling approach for measuring the DEPFET source-drain current has to be chosen to achieve fast read-out times. For a 768 pixels long matrix, it is thus necessary to read out four adjacent rows at once. This increases the thermal dissipation of the DEPFET pixels to about $500 \mathrm{~mW}$, which is still manageable by a passive cooling only. This imposes the requirement to process 1000 drain currents in parallel in time window of about $100 \mathrm{~ns}$.

Dedicated application-specific integrated circuit (ASIC) chips have been designed and produced to fulfil the tasks of steering the rolling-shutter read-out, sampling and digitising the DEPFET drain currents and digital data processing, as well as providing an off-module interconnection. These ASICs are introduced in the following.

Rolling-shutter Driver (SWITCHER) The SWITCHER ASIC is implemented in the AMS $0.35 \mu \mathrm{m} \mathrm{HV}$ technology with special measures ensuring radiation hardness and controls the rolling-shutter read-out sequence. It provides fast $(\approx 10 \mathrm{~ns})$ voltage swings of up to $20 \mathrm{~V}$ on its outputs. One SWITCHER chip provides 32 output groups, where one output group comprises one gate- and one clear-driver. These drivers provide the $V_{\text {gate-off }} \leftrightarrow V_{\text {gate-on }}$ voltage swing for activating the DEPFETs for sampling their drain currents, and the $V_{\text {clear-off }} \leftrightarrow V_{\text {clear-on }}$ voltage swing for clearing the internal gates, respectively. Combining four rows of pixels in one rolling-shutter step, this means one SWITCHER is capable of steering 128 DEPFET pixel rows. The ASIC design allows for daisy-chaining multiple chips, with a rolling-shutter sequence shifting through all consecutive chips before repeating. For steering a 768 row pixel matrix, six SWITCHER ASICs are necessary. The precise timings and lengths of gate and clear sequences are controllable by dedicated STROBE signals. Radiation hardness up to $36 \mathrm{Mrad}$ has been demonstrated [29].

Drain Current Digitizer (DCD) The Drain Current Digitizer (DCD) is implemented in the UMC $0.18 \mu \mathrm{m}$ CMOS technology with design techniques improving the radiation hardness. It provides inputs for drain currents of DEPFET pixels. The DCD samples the drain currents and digitises them with 8-bit resolution, yielding a value in the range 0 to 255 in arbitrary digital units (ADU). One DCD chip provides 256 input channels, where one channel comprises an input receiver that keeps the drain potential constant and provides a programmable current amplification, followed by a pipeline analogue-digital converter (ADC). Each channel samples and digitises input currents every $100 \mathrm{~ns}$. The 


\section{A DEPFET-based Pixel Detector for Belle II}

\begin{tabular}{cc}
\hline$g_{\mathrm{DCD}}[\mathrm{nA} / \mathrm{ADU}]$ & dyn. input range $[\mu \mathrm{A}]$ \\
\hline $75 \pm 8$ & $19 \pm 2$ \\
$96 \pm 10$ & $24 \pm 3$ \\
$140 \pm 10$ & $37 \pm 4$ \\
$170 \pm 20$ & $44 \pm 4$ \\
\hline
\end{tabular}

Table 6.2.: DCD input amplification gains and the corresponding effective dynamic input range.

input range of each $\mathrm{ADC}$ is $0 \mu \mathrm{A}$ to $32 \mu \mathrm{A}$. The effective dynamic input range of the channels is determined by a globally adjustable input amplification gain factor $g_{\mathrm{DCD}}$ of the receivers. Table 6.2 lists the gain factors and the corresponding effective dynamic input range. Adjustable global (identical for all 256 channels) and dynamic (for each channel and four-row block individual) current sources and sinks are available for shifting the input currents in the dynamic range. The purpose and usage of these are discussed in [51]. Additionally, an analogue common mode fluctuation ${ }^{4}$ compensation (ACMC) is implemented. Each channel can individually be selected to contribute to and receive the common mode correction. The analogue part of the chip generates a significant thermal power dissipation of up to $2 \mathrm{~W}$ per chip.

An additional digital logic block serialises the digital outputs and sends them out via eight 8-bit-parallel lines at $400 \mathrm{Mbit} / \mathrm{s}$. Configuration of the various current sources and sinks as well as the amplification and digitisation modes is possible via a slow control interface $^{5}$. The DCD ASIC has an expected radiation tolerance of 20 Mrad [52].

For reading out the 1000 DEPFET pixels in each four-row block in parallel, four DCD ASICs are used. Six channels in each chip are not connected to any drain line and have a floating potential at the input pads. The unconnected channels are not considered in common mode corrections and their digitised data is discarded. The drain lines connecting the DEPFET drain contacts and the DCD channel inputs run columnlike through the pixel matrix. Thus, one drain line connects one DCD channel to 192 DEPFET cells that are consecutively sampled. The combination of channel number and rolling-shutter sequence position uniquely identifies each pixel in the matrix.

Data Handling Processor (DHP) The Data Handling Processor (DHP) is implemented in the TSMC $65 \mathrm{~nm}$ technology. Its purpose is the compression of the high data rates produced by the DCD chip. Due to bandwidth limitations of the off-module interconnection, it is not feasible to transmit the full digitisation data. Digitised pixel signals received by the DCD are zero-suppressed, all signals below an adjustable digital threshold are discarded. The threshold is exclusive, that means a zero-suppression threshold of $7 \mathrm{ADU}$ yields signals $\geq 8 \mathrm{ADU}$. The chip provides memory for pixel-wise digital

\footnotetext{
${ }^{4} \mathrm{~A}$ common mode fluctuation is a signal variation that affects all inputs in the same way.

${ }^{5}$ Slow control interfaces for configuration use the Joint Test Action Group (JTAG) standard (IEEE standard 1149.1).
} 


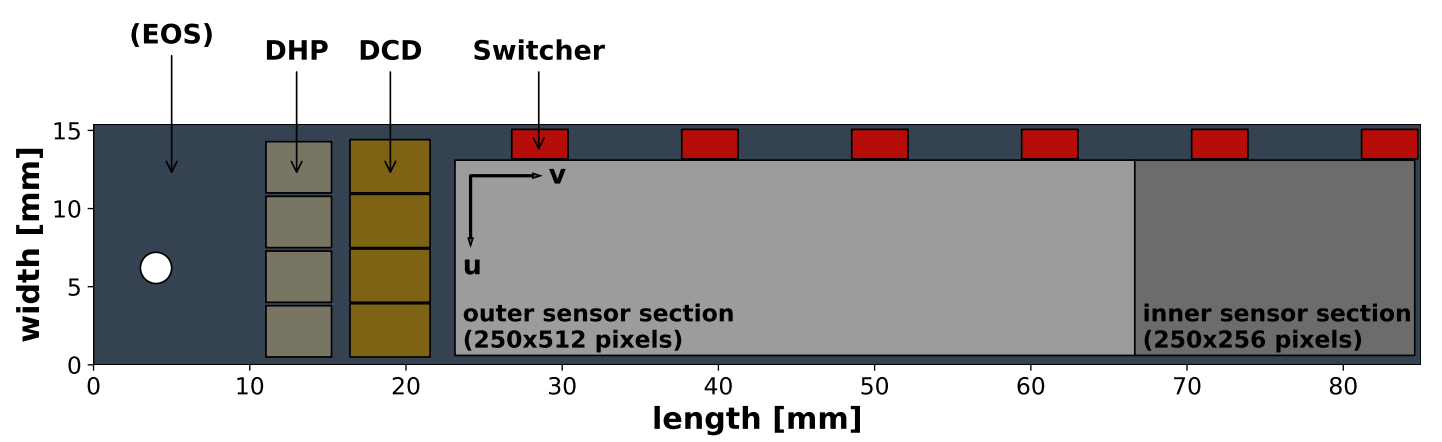

Figure 6.5.: Sketch of the layout of a detector module for the Belle II pixel detector.

offset subtraction before zero-suppression is applied. Additionally, a digital common mode correction (DCMC) can be activated and is applied before zero-suppression. Zerosuppressed signal information - that comprises the column and row id, the digitised signal value above threshold and the applied DCMC value - is stored in memory. Data is only sent off-module upon receipt of a trigger signal from the back-end system. The DHPs ensures proper functionality and data persistence when at maximum $3 \%$ of the $250 \times 768$ pixels exceed the zero-suppression threshold. The threshold has to be chosen appropriately. One DHP ASIC provides the inputs for connecting to one DCD chip. Thus, in total four DHP chips are used for read-out.

Despite data processing, the DHP also serves as a controller for all on-module chips. It presents the primary interface to the back-end system, forwarding configurations to the respective ASICs. The DHP also provides a set of fast control signals that are distributed to DCD and SWITCHER chips for synchronisation. The off-module data links run at $1.25 \mathrm{Gbit} / \mathrm{s}$.

\subsubsection{Module design}

One of the central design guidelines for the Belle II pixel detector is the minimisation of the material budget of the full detector, including support and cooling structures. For the single detector modules, this results in an all-silicon device design. The DEPFET pixel matrix is implanted on a $75 \mu \mathrm{m}$ thin piece of silicon. This sensor silicon is directly bonded onto a silicon frame of $450 \mu \mathrm{m}$ thickness. This frame serves as a mechanical stabilisation of the module. Supply lines for power and data interconnection are directly routed in three metal layers (made of $\mathrm{Al}$ and $\mathrm{Cu}$ ) in the silicon. For minimising the material budget, the support wafer material directly below the sensitive volume of the DEPFET pixel matrix is removed. The SWITCHER ASICs are placed on a $2.3 \mathrm{~mm}$ wide balcony alongside the 768 pixels extent of the matrix. The balcony has a thickness of $525 \mathrm{\mu m}$. Groves are etched from the backside of the balcony to further reduce the overall material budget in the detector acceptance. Figure 6.5 shows a sketch of the module layout. The short side is extended by about $23 \mathrm{~mm}$. This region is called the end-of-stave (EOS) and the DCD and DHP read-out ASICs are placed there. Since 


\section{A DEPFET-based Pixel Detector for Belle II}

\begin{tabular}{ccc}
\hline PXD layer & inner section & outer section \\
\hline 1 & $50 \mu \mathrm{m} \times 55 \mu \mathrm{m}$ & $50 \mu \mathrm{m} \times 60 \mu \mathrm{m}$ \\
2 & $50 \mu \mathrm{m} \times 70 \mu \mathrm{m}$ & $50 \mu \mathrm{m} \times 85 \mu \mathrm{m}$ \\
\hline
\end{tabular}

Table 6.3.: Pixel pitches of the DEPFET pixel detector modules. Given are the pitch in the $u$-direction (short module side, 250 pixels) and in the $v$-direction (long module side, 256 inner and 512 outer pixels, respectively).

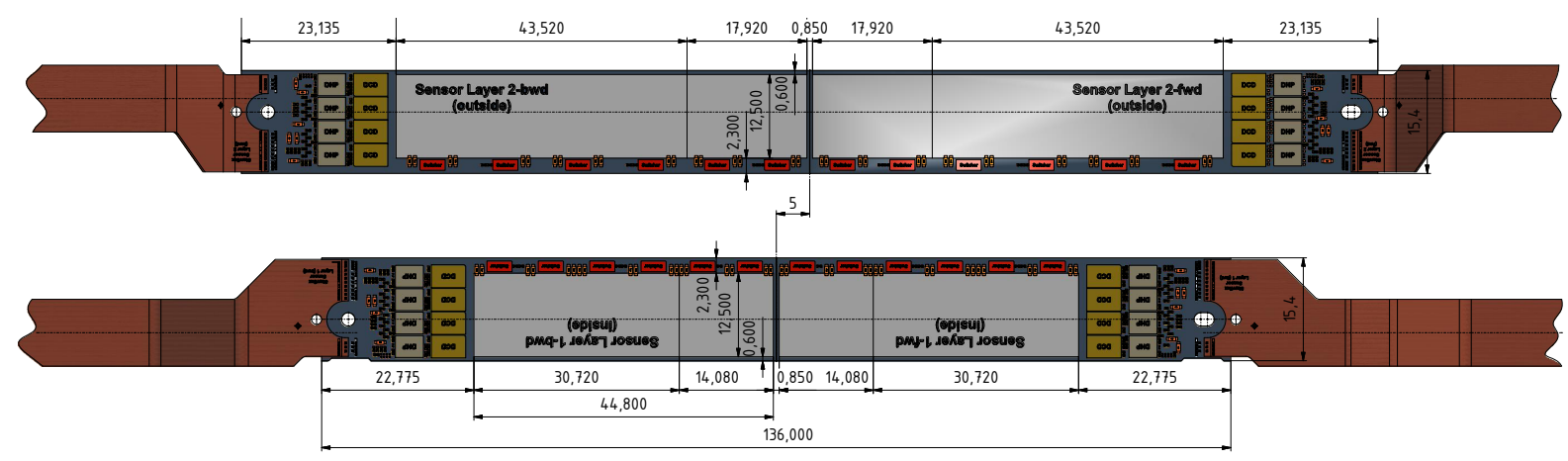

Figure 6.6.: Technical drawing of the first layer (bottom) and second layer (top) ladders for the pixel detector with the four distinct module types. Figure is from [53].

the EOS region is outside of the nominal detector acceptance, active cooling of the read-out ASICs is possible. All ASICs are directly bump-bonded on the silicon frame. A flexible multi-layer polyimide printed circuit board (Kapton) is soldered and wirebonded to the EOS and routes all analogue supply lines (DEPFET potentials, ASIC supply) and digital communication lines (JTAG slow control and off-module data links), connecting the module and the power-supply system and the back-end electronics. There are additional passive components, resistors and capacitors, soldered as surface-mounted devices (SMDs) directly onto the silicon as well.

The pixel matrix is partitioned in an inner and an outer section. The inner section (rows 512 to 768 ) covers the central angular acceptance. Particles emerging from the IP in a the central angular acceptance traverse the PXD modules with a close to perpendicular angle. The DEPFET pixels in the inner section are thus designed with a smaller pitch in the respective direction. The outer section (rows 1 to 512) covers the forward (backward) region with more inclined particle incidences and larger pixel pitch. Moreover, the second layer's pixel pitches are larger than the first layer pitches. Table 6.3 summarises the various pixel pitches used in the PXD design. Due to the differing pixel pitches, the overall geometrical size of first and second layer modules differs as well. Since two modules are glued back-to-back to form a ladder of the pixel detector, in total four different module designs exist and need to be produced. Figure 6.6 shows a technical drawing of the first and second layer ladders.

The DEPFET supply voltages as listed in Table 6.1 apply to all pixels of the module. 


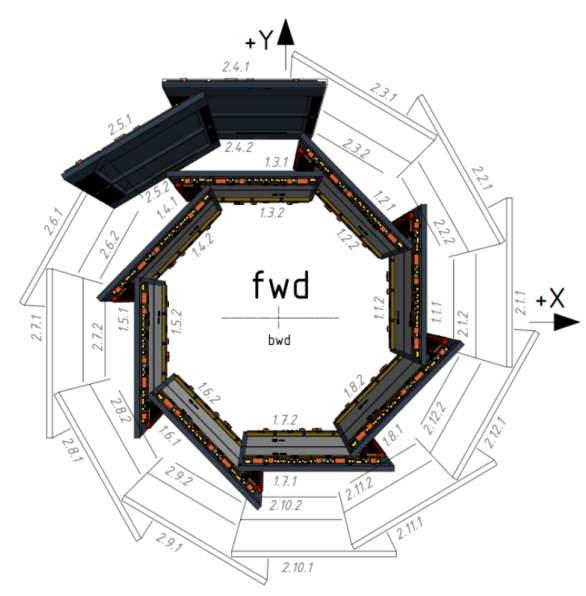

Figure 6.7.: Windmill arrangement of the pixel detector's inner and outer ladders. At the start of the data taking period in 2019 , only the indicated ladders were installed. Figure is from [54].

To be able to fine-tune the characteristics of the outer section and inner section pixels and to cope with possible inhomogeneities - induced for example by an angular dependence of the expected irradiation background -, the matrix is electrically divided in row direction into three domains. Each domain has an independent $V_{\text {gate-on }}$ and $V_{\text {cleargate }}$ potential. The domains are enumerated starting at the EOS, with the first domain spanning rows 1 to 256 , the second domain spanning rows 257 to 512 and domain three spanning rows 513 to 768 .

\subsection{The Belle II pixel detector}

The inner and outer ladders are arranged in a windmill structure to form the full pixel detector for Belle II as sketched in Figure 6.7. The eight inner ladders are positioned with the front-side (where the ASICs are mounted) facing towards the IP. The twelve outer ladders are facing outwards. The overall material budget of per layer of the PXD is $0.21 \%$ of a radiation length. The thermal power dissipation of the DEPFET pixel matrix and the SWITCHER ASICs is sufficiently low to apply a passive cooling by a constant flow of $\mathrm{N}_{2}$ gas. The read-out ASICs at the EOS, however, require active cooling. The ladders are mounted on both ends on a support cooling block. This is a Parylene-coated stainless steel structure with internal cooling channels. The ladders are screwed directly onto the support structure. The coating prevents any electrical contact, but establishes a thermal contact between the EOS of the modules and the structure. The internal channels are flushed with two-phase $\mathrm{CO}_{2}$ resulting in evaporative cooling. The total thermal power dissipation of the pixel detector is in the order of $360 \mathrm{~W}$.

The operation of the modules in the pixel detector requires a dedicated service infrastructure. A power supply unit per module provides the necessary DEPFET biasing and ASIC operation potentials and currents. Each module digitally communicates with 


\section{A DEPFET-based Pixel Detector for Belle II}

a separate back-end device, which sends trigger to and receives data from the module. In addition, the configuration of the on-module ASICs (slow control) is steered by this back-end device. Additional data processing layers and devices exist in the back-end and provide the interoperability with the other Belle II detectors and data processing systems. Details of the services that are required to operate a single module in a laboratory environment are discussed in the following chapter.

At the start of the data taking period of Belle II in 2019, the complete inner layer, but only one sixth of the outer layer modules were installed (cf. Fig. 6.7). The reasons are delays in the process of glueing modules to layers. Initial results demonstrate module hit efficiencies above $98 \%$ [54] and even with the reduced PXD, a transverse impact parameter resolution of 14.1(1) $\mu \mathrm{m}$ [39]. 
CHAPTER 7

\section{Series Testing of Pixel Detector Modules for Belle II}

The Belle II pixel detectors is built from 40 modules - eight of each inner layer type for the eight inner ladders and twelve of each outer layer type for the twelve outer ladders. Before being installed in the detector, each module is tested for functionality and its operation parameters are characterised and optimised to assess its qualification. In fact, more than 40 modules need to be assembled and tested in order to account for a less than $100 \%$ yield. The pixel detector modules with its 14 ASICs of three different types, each with numerous tunable operation parameters, and its DEPFET sensor matrix with the various biasing potentials is a very complex device. The optimisation of the module performance by tuning of the operation parameters hence is a challenging task. The series testing of modules is an important step and a significant effort on the way to the installation of the pixel detector in Belle II. This chapter briefly outlines the module production process from the wafer level sensor production to the complete module assembly. The complex characterisation and optimisation procedure is explained and representative results of one module are shown. A procedure for finding the optimal combination of DEPFET biasing potentials is proposed. Finally, an overview and summary of the set of modules that were tested in the laboratory in Göttingen are presented to examine the consistency of the optimisation procedure and to assess intrinsic variations of characteristics among modules.

\subsection{Production of Belle II pixel detector modules}

The production of the modules for the Belle II pixel detector involves several steps, from the wafer level sensor production to the bump-bonding of the read-out ASICs and the assembly to an operational device, conducted at several locations. Various processing and assembly steps are necessary and are briefly sketched here. Figure 7.1 sketches the sensor production on wafer level. Wafers of the production run are manufactured by 


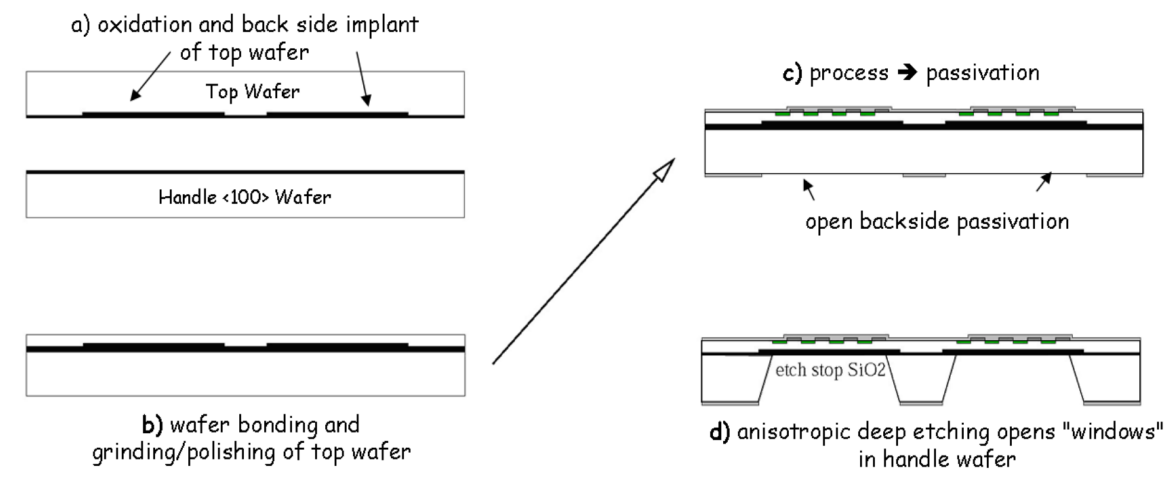

Figure 7.1.: Sketch of the processing steps of the wafer production for pixel detector modules. A top wafer (a) is $\mathrm{p}^{+}$implanted on the backside to form the backside implant of the DEPFET cell (cf. Fig. 6.1) and is subsequently (b) bonded from the backside to a handle wafer with an oxide layer in between. The top wafer is thinned to $75 \mu \mathrm{m}$ by grinding and polishing on the top side. Implants on the top side (c) form the DEPFET cell matrix. Metallisation layers are applied on top. A passivation layer is added and opened below the $\mathrm{p}^{+}$implants on the backside of the handle wafer. Anisotropic deep etching is used (d) for thinning down the handle wafer below the DEPFET cells from the backside, stopping at the $\mathrm{p}^{+}$backside. Figure is from [29].

IceMOS ${ }^{1}$ and Shin-Etsu ${ }^{2}$, and are processed at the Halbleiterlabor of the Max-PlanckSociety (HLL). A first quality assessment of implantations and electrical connections is done on wafer level. Subsequently, the wafer is cut into the individual parts. Each wafer yields six "bare-bone" detector modules and additional test structures. A wafer houses one module of type inner layer forward (IF), one of type of inner layer backward (IB), two of type outer layer forward (OF) and two of type outer layer backward (OB). A module is labelled by its two digit production wafer number and by its type (e.g. W40_IF). For the outer layer modules, the number 1 or 2 is added (e.g. W11_OF2), depending on the position on the wafer.

The read-out ASICs are produced separately in their respective technology. Each ASIC is individually tested for functionality on a needle card test stand. The six SWITCHER ASICs, four DCD ASICs and four DHP ASICs are bump-bonded and flip-chipped to the module. In addition, several SMD components (resistors and capacitors) are soldered onto the module. Before the off-module flexible PCB is soldered and wire-bonded to the module, a probe card test is applied. The probe card contacts the off-module interconnection pads and basic functionality of the module is verified [55]. Modules passing the probe card test are finally equipped with the off-module flexible PCB connection.

\footnotetext{
${ }^{1}$ IceMOS Technology Ltd, 5 Hannahstown Hill, Belfast BT17 0LT

${ }^{2}$ Shin-Etsu Chemical, 6-1, Ōtemachi 2-chome, Chiyoda-ku, Tokyo
} 


\subsection{Testing and characterisation procedure}

Once a pixel detector module is fully assembled and passed the needle card test, it undergoes a thorough characterisation procedure. The functionality of the digital parts, like ASIC configuration interfaces or digital data transmission, is tested. Analogue parts, like the distribution of pedestal values, read-out noise or charge collection properties, are characterised and optimal, module-specific operation parameters are determined. Based on the results, a module grade is assigned. The module grade is considered when deciding upon the further utilisation of the module. The pixel detector module testing and characterisation is conducted in standardised laboratory environments and setups. The setup is explained in the following section. Several institutes in the Belle II pixel detector collaboration contributed to the series testing of modules. The standardised characterisation and grading program is explained following one example module, labelled W11_OF2, through the procedure. The scope of this thesis was to contribute to developing and establishing this program and applying it to modules at the Göttingen test stand. Overall, 17 modules were processed in Göttingen.

\subsubsection{Laboratory setup}

The pixel detector modules are operated in dedicated, small-scale laboratory setups. These are small-scale in the sense that only single modules with their required infrastructure are operated compared to the full Belle II pixel detector, where 40 modules are operated in parallel. Identical setups are available at the different series testing sites. Reference modules are operated and measured at all these locations in order to qualify each setup and verify that the measurement results are in agreement.

The setup neeeds to be placed in a controlled laboratory environment. Particularly, modules must only be unpacked from the transport housings and operated openly in a cleanroom environment. Contamination of the sensor area by dust particles is thus avoided. Furthermore, the humidity must be controlled in order to prevent water condensation on the module. While other testing sites provide a dedicated cleanroom, the test stand in Göttingen employs a laminar flow cabinet supplied with filtered air. Modules are stored in a dry cabinet with controlled humidity when not installed in the setup. The laboratory space in Göttingen does not provide a general humidity controlled ventilation. The operation thus has to be ceased while the measured humidity is too high. The Göttingen test stand comprised two identical and independent module operation setups. The operation of a Belle II pixel detector module requires a specific infrastructure consisting of a set of custom-made components and some regular consumer-grade components. A sketch of the constituents and their respective interconnections is shown in Fig. 7.2.

Mechanical mounting The module is placed on a parylene-coated piece of aluminium referred to as transport jig. The coating prevents any electrical contact between the module and the jig, while a thermal contact is established. The flexible Kapton interconnection is retained in an aluminium holding structure. A 3D-printed polymer clamp 


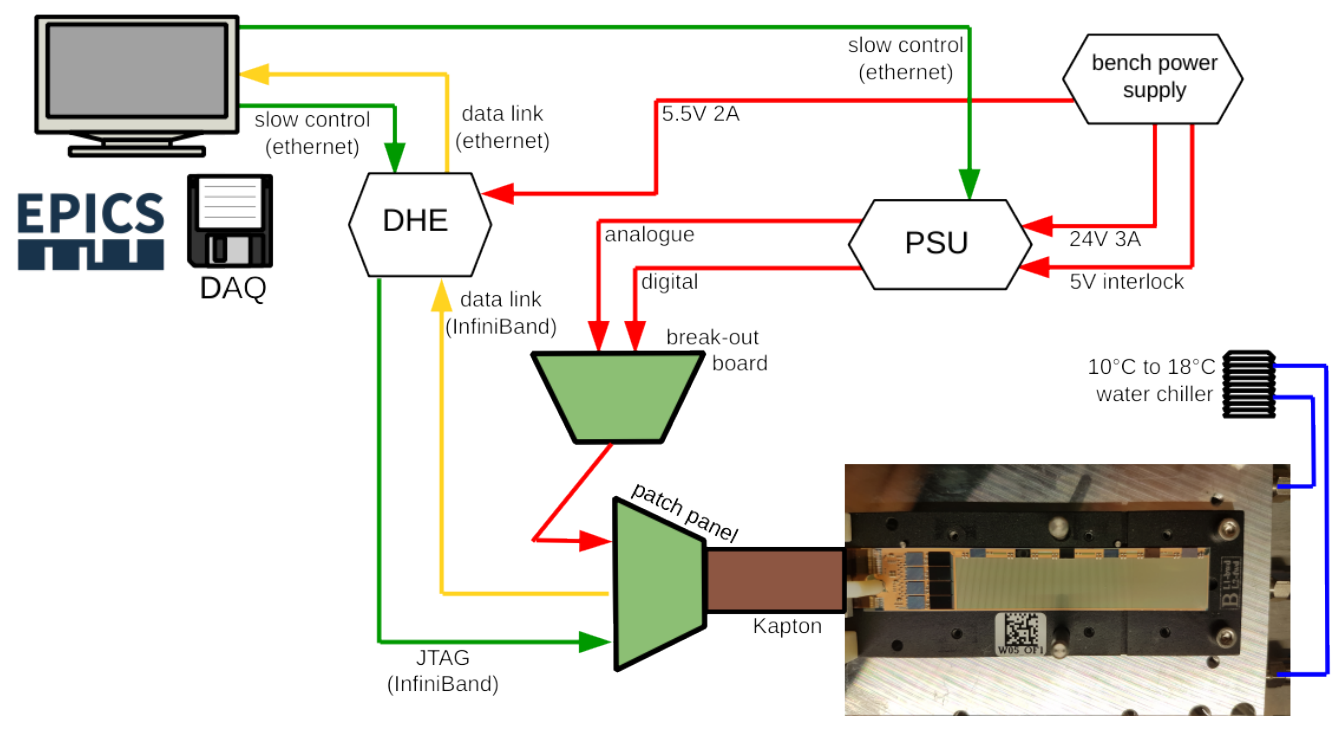

Figure 7.2.: Sketch of the components and interconnections of the laboratory setup for operation of a pixel detector module.

is screwed into the transport jig and slightly presses the module onto the jig in the area of the EOS. This improves the thermal contact between module and jig at the EOS. Grooves are cut out in the transport jig below the sensor area that allow for the application of a soft under-pressure (a few 100 mbar) which improves the thermal contact along the balcony. The transport jig is in turn screwed to a cooling jig. This is a piece of aluminium with an internal cavity that is constantly flushed with cooled water. The water temperature is in the range of $10^{\circ} \mathrm{C}$ to $18^{\circ} \mathrm{C}$. Figure 7.3 shows a picture of the mechanical mounting.

The DHP ASIC provides a measurement of the on-chip temperature via measuring a voltage drop over a sensing diode. Although the absolute value of the temperature reading is very sensitive to the DHP supply voltage, the measurement is trusted within

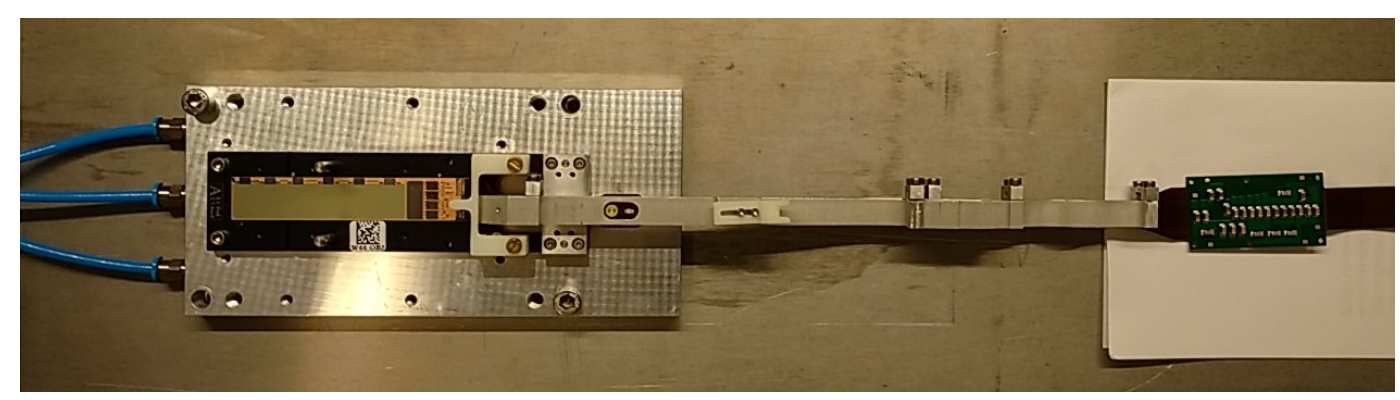

Figure 7.3.: Picture of the mechanical mounting of a module on the transport jig (black coating) and on the cooling jig (metallic). The flexible Kapton interconnection is fixated in an aluminium holding structure. 
$5{ }^{\circ} \mathrm{C}$ accuracy. A temperature reading below $80{ }^{\circ} \mathrm{C}$ is considered safe for operation. Especially changes of the temperature reading over time are monitored. A heat-up of the DHP ASICs indicates an overall insufficient thermal contact and further operation poses a danger to the module.

Power supply The voltages and currents required by the read-out ASICs and the DEPFET sensor are supplied to the module by a custom-built 24-channel power supply unit (PSU) [56]. The flexible Kapton interconnection coming from the module is connected to a printed circuit board (PCB), referred to as patch panel. There, the individual connections are split up into three domains with dedicated connectors: the high-speed data connection, the configuration interface and the power supply. The latter connects to another PCB, referred to as break-out board via a custom-built 51-wire cable of about $50 \mathrm{~cm}$ length. The break-out board splits the power supply lines into two domains with dedicated connectors: analogue supplies and digital supplies. These two domains are routed to the PSU via two identical, custom-built cables of $14 \mathrm{~m}$ length that integrate appropriate shielding against external electro-magnetic disturbance. The PSU provides the two interconnections for the two module supply domains, a consumer-grade ethernet connection for configuration and control, two banana connectors for providing a $24 \mathrm{~V}$ input at up to $3 \mathrm{~A}$, and a special connector receiving a $5 \mathrm{~V}$ active interlock signal. In the laboratory, the $24 \mathrm{~V}$ and $5 \mathrm{~V}$ inputs are provided by a consumer-grade bench power supply ${ }^{3}$.

For mitigating voltage drops over the various cables and connectors, the potentials are sensed as close to the load as possible and the PSU output is adjusted to reach the target potential at load. In order to prevent damage to electrical components by high currents, the output currents are limited. Targeted load potentials and current limits can be adjusted by the slow control. The power-up of a module is done in several steps. First, the ASIC supply voltages are applied at low current limits to establish the configuration interface. The ramp-up of the SWITCHER supply potentials require an interleaved stepping. Once the ASICs are properly configured, the current limits are raised. Next, the DEPFET biasing and switching potentials are applied in a specific sequence.

Data collection and configuration back-end The data output of a module is processed by the Data Handling Hybrid (DHH) system [57]. The Data Handling Engine (DHE) is a field-programmable gate array (FPGA) based device forming the primary back-end in a one-to-one correspondence ${ }^{4}$. The high-speed data interconnection from the DHPs to the DHE, via the Kapton and patch panel, is routed in InfiniBand cables of $1.5 \mathrm{~m}$ length. The DHE also provides the JTAG configuration interface and distributes trigger signals

\footnotetext{
${ }^{3}$ The Göttingen setup uses the model HMP4040 by HAMEG Instruments/Rohde \& Schwarz.

${ }^{4}$ There is one DHE for each module in the final Belle II pixel detector summing up to 40 DHEs. Five DHEs are multiplexed and connected to and managed by one Data Handling Concentrator (DHC) and one Data Handling Interface (DHI). These are connected to further back-end systems. In the laboratory setup, DHC and DHI are not used.
} 


\section{Series Testing of Pixel Detector Modules for Belle II}

to the module's ASICs via an ethernet-type interconnection. The device itself is a PCB implemented with the advanced mezzanine card (AMC) standard. In the laboratory setup, it is placed in a carrier board ${ }^{5}$ that provides direct ethernet connections to a desktop computer. The carrier board is supplied with $5.5 \mathrm{~V}$ at about $1 \mathrm{~A}$ to $2 \mathrm{~A}$ from the bench power supply.

The desktop computer receives data from the DHE over an ethernet connection via the user datagram protocol (UDP), which is processed and written to disk by a custom data acquisition software (DAQ). Trigger signals can be generated in the DHE internally at a rate of $1 \mathrm{~Hz}$ to $10 \mathrm{kHz}$ for stand-alone laboratory operation. The DAQ provides a fraction of the recorded data in real time for live data quality monitoring.

Slow Control Another ethernet connection between the desktop computer and the DHE provides the configuration interface. This is used to configure the DHE itself, regarding for example the trigger generation, and for configuration of the read-out ASICs on the module. Technically, the configuration interface is implemented in the control frame-work EPICS [58]. The desktop computer runs pieces of software called inputoutput controllers (IOCs). The IOCs provide several channels, representing configurable switches (e.g. trigger on/off) or values (e.g. supply voltage), which can be manipulated from within the software performing the module testing procedure or via a graphical user interface (GUI). The values of all channels are constantly monitored and recorded in a database. Time series of for example current consumption of individual supply lines are thus available for investigating the behaviour of modules during the testing program.

\subsubsection{Digital communication}

The digital communication comprises three different communication domains that are checked for functionality after a module is installed in the test stand. These are

- the JTAG interface for configuring the ASICs,

- the DCD-to-DHP transmission of digitised DEPFET currents, and

- the off-module DHP-to-DHE high speed data link.

At the first stage of the module power-up, the ASIC supply voltages are applied at reduced current limits to ensure that any initial misconfiguration does not cause a damaging amount of internal currents. All 14 ASICs on the module are daisy-chained in one configuration JTAG-chain. The DHE acts as the primary JTAG-port initialising the chain. The JTAG interface of each ASIC type provides an ASIC-specific identification code. The functionality of the full chain is tested by checking the correct receipt of all identification codes. Once the appropriate (initial default) configurations are written to all ASICs, the current limits are raised to nominal operation values. In case the

\footnotetext{
${ }^{5}$ In the final experiment, the AMCs are located in AMC-carriers in advanced telecommunications computing architecture (ATCA) shelfs. There, the configuration interface to the module is solely provided by the DHI.
} 
JTAG-chain cannot be established successfully, the module fails the test. Due to the daisy-chaining, a single ASIC with for example a faulty bump-bond can cause a failing of the JTAG configuration test.

The 8-bit digitised DEPFET currents are transmitted from one DCD chip to one DHP chip via 64 individual data lines. The DCD can be configured to send a fixed and welldefined test pattern over the data lines. With this mode enabled, the data received by the corresponding DHP is compared to the test pattern. The pattern is non-uniform over the 64 lines and varies over 32 clock-cycles before repeating. Any deviation indicates an incorrect data transmission. Since the 64 lines per pair of DCD and DHP ASIC are not routed uniformly within the modules metal layers, the overall line length or the strength of capacitive coupling to surrounding structures can differ among lines. Signal propagation times can thus differ as well and transmission errors are likely. In order to mitigate this effect, a configurable signal delay can be added to each line individually (local delay). The delay is realised by propagating the signal through a number of zero to 15 identical inverter elements at the receiver side. Additionally, the point in time where the line potentials are sampled at the DHP (sampling point), common for all 64 lines, can be delayed analogously (global delay). This results in a two-dimensional (local delay - global delay) parameter space of $16 \times 16$ settings per data line of every DCD-DHP pair. At each parameter space point, the test pattern sequence is recorded for several repetitions and the relative occurrence of bit transmission errors is determined. Starting from a global delay of zero, a local delay setting for each of the 64 data lines yielding zero transmission errors is searched for. If no error-free transmission setting for any data line is found, the global delay is increased by one. Once a valid combination of global and local delays is found, the optimisation is stopped. This is repeated for each of the DCD-DHP pairs. If no error-free transmission can be achieved for one or more data lines on one ore more ASIC pairs, for example due to a bad bump-bond, the module fails the test. Figure 7.4 shows the averaged number of transmission errors (bit errors) per local delay setting for the 64 data lines of one ASIC pair.

The data transmission from DHP to DHE is implemented in the Aurora 8b/10b protocol by XILINX, which is a serial connection-oriented link-layer communication protocol using 8-bit-to-10-bit encoding. The data stream from each DHP has an effective data rate of about $1.2 \mathrm{Gbit} / \mathrm{s}$. The DHP uses a current mode logic (CML) driver for transmitting data. For mitigating signal degradation effects over the physical connection, a programmable signal pre-emphasis is implemented in the driver. The strength and the length of the pre-emphasis is adjustable in discrete steps. The optimal values are found by scanning the parameter space and testing whether the high speed link is successfully established and stable aver a certain time period, usually $1 \mathrm{~s}$ to $5 \mathrm{~s}$, for each configuration. This is done for each DHP chip individually. However, these optimal parameters depend strongly on the individual setup due to differing cable lengths. All four data links are constantly monitored and an online reset is possible in case of link losses. If no configuration with stable data links can be identified, the module is set aside for further investigation. The initial qualification of each setup using reference modules ensures that link problems do not arise from external sources of disturbance at the local laboratory. 


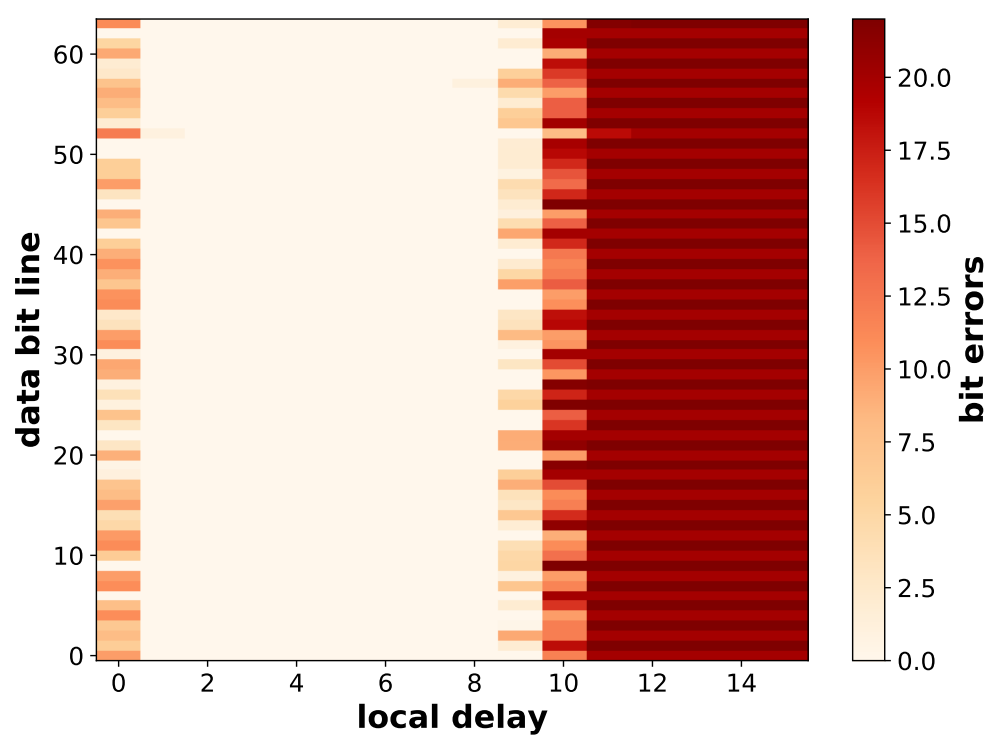

Figure 7.4.: Example of the DCD to DHP transmission delay optimisation. The global delay is zero. A local delay setting of 4 to 5 yields an error-free transmission for each of the 64 data lines.

\subsubsection{Analogue-to-digital conversion}

The DCD ASICs implement the analogue-to-digital conversion (ADC) of the DEPFET drain currents. The currents, in the order of about $100 \mu \mathrm{A}$, are translated to an 8-bit digital value in the range 0 to 255 in arbitrary digital units (ADU). The analogue-todigital conversion is required to be linear over the full dynamic range. The DCD offers a range of programmable parameters that steer the operation of the $256 \mathrm{ADC}$ channels in the chip. Out of these, four parameters that define the strength of current sources/sinks have been identified to have the most significant influence on the AD conversion. They are labelled Src1, Src2, Src3 and Bias ${ }^{6}$. These current sources/sinks are adjustable by corresponding digital-to-analogue converters (DACs). In addition, the DCD supply voltages $V_{\text {RefIn }}$ and $V_{\text {AmpLow }}$ have an influence on the analogue-to-digital conversion characteristics. The optimal set of parameters and voltages has to be identified at which all ADCs produce acceptable conversion characteristics. This set of parameters is not necessarily identical for all four DCD chips on the same module or among different modules.

The tuning of the six operation parameters per DCD ASIC is a challenging task. An iterative optimisation procedure has been developed in [59]. It scans the parameter space in one- and two-dimensional slices ${ }^{7}$. For each combination of parameters, the analogue-

\footnotetext{
${ }^{6}$ In the DCD manual and the slow control interface these are called IPSource, IPSourceMiddle, IPSource2 and IFBPBias.

${ }^{7}$ Scanning of the full six-dimensional parameter space is too time consuming.
} 
to-digital transfer curve (ADC curve) for each ADC is recorded. The analogue input is varied over the full dynamic range and the corresponding digital output is registered. The ADC curves are checked for integral (INL) and differential (DNL) non-linearity and conversion fluctuations (noise). Ideally, both non-linearity measures are zero and the transfer curve covers the full dynamic input range. Also the noise should be minimised. Details on the measurement and complex optimisation procedure can be found in [59].

\subsubsection{Pedestal distribution and read-out noise}

The source-drain current $I_{S D}$ of a DEPFET pixel increases when charges are collected in the internal gate. The base value, when no charge is present, is called pedestal current $I_{\text {ped }}$. It depends on the gate potential $V_{\text {gate-on }}$ as well as on intrinsic variations due to the wafer production process. The absolute values of the pedestal currents of all DEPFET pixels in the matrix are not providing any useful information. However, since the pixel rows are read out sequentially and their drain currents are consecutively fed into the ADCs, the relative spread of the pedestal currents is of concern. The dynamic input range of the ADC channels must cope with the spread in pedestal values and must provide sufficient headroom for actual signal currents on top of the pedestal currents. At the same time, the current digitisation must provide a sufficient granularity in order to yield information on the strength of signals ${ }^{8}$. Each DCD ASIC implements a programmable current sink, $I_{\text {global }}$, for subtracting a global offset of all $I_{\text {ped }}^{i}$, where $i$ enumerates the DEPFET pixels read out by the respective DCD. It is chosen to be just below $\min _{i} I_{\text {ped }}^{i}$. In addition, a mechanism for local (i.e. per pixel) offset compensation is implemented for compressing the width of the pedestal current spread. A programmable current sink, $I_{\text {local }}$, is used to subtract $n_{\text {local }} \in[0,1,2,3]$ times the amount of $I_{\text {local }}$ from $I_{\text {ped }}^{i}$. The number $n_{\text {local }}$ can be chosen for each pixel in the matrix individually. This is effectively a DAC of 2-bit width, where the 2-bit values for each pixel are stored in a memory in the DHP ASIC and transmitted to the DCD. Details on how these offsets are optimised can be found in $[48,51]$.

The ADCs in the DCD thus digitise the residual pedestal current plus a possibly present signal current of each DEPFET cell. The DHP ASIC processes the digital data and performs zero-suppression to only consider signals above a certain threshold. The residual pedestal portion has to be subtracted on the digital level before zero-suppression is applied. The pixel-specific residual pedestal values are stored in a memory on the DHP chip. To calibrate these values, the DHP implements a raw data read-out mode at low frequency. In this mode, the digitised current values for each DEPFET pixel are directly passed to the back-end system without applying DHP-level pedestal subtraction or zero-suppression. These raw values are repeatedly read-out 100 to 1000 times and averaged. The average and 8-bit integer rounded pedestal values are uploaded to the DHP memory and used for pedestal subtraction. The standard deviation over the raw

\footnotetext{
${ }^{8}$ The read-out concept was chosen to provide information on the amount of charge collected by each pixel beyond a pure binary hit/no hit measurement. This improves the spatial resolution by considering the relative signal strength in neighbouring pixels. If the digitisation granularity is too coarse, the improvement weakens and the spatial resolution becomes worse. This will be discussed in Chapter 8 .
} 


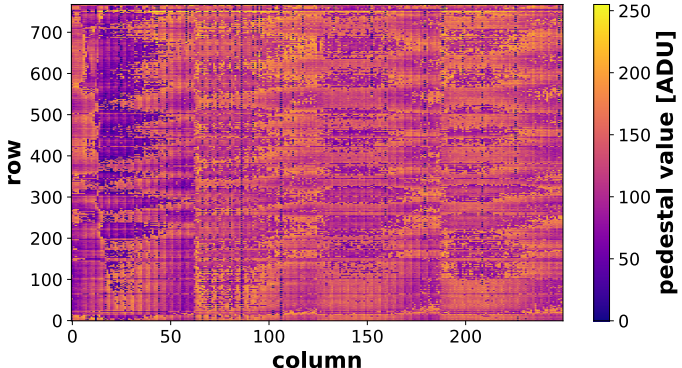

(a)

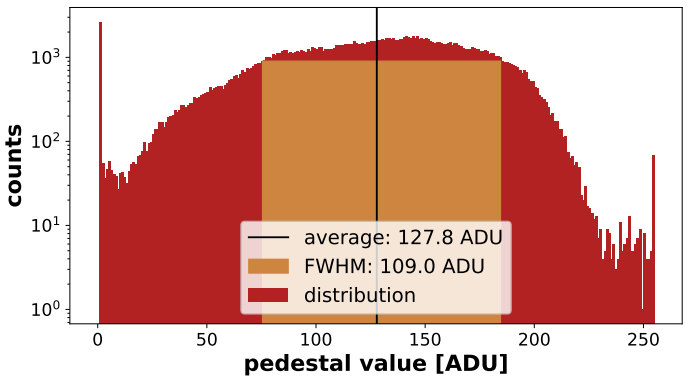

(b)

Figure 7.5.: Pedestal values as a function of the column and row coordinate (a) and distribution (b) over the $250 \times 768$ DEPFET pixels of module W11_OF2. Multiple problematic drain lines are visible as regular patterns along columns (see text). The values are histogrammed in bins of $1 \mathrm{ADU}$ width. The lowest bin collects non-functional pixels and is not considered for the computation of the FWHM.

values, the pedestal fluctuation, is used as a measure for the pixel-specific read-out noise. This intrinsically includes contributions from the analogue-to-digital conversion in the DCD. In fact, this contribution dominates the effective read-out noise [45].

A module can be characterised in terms of the remaining effective pedestal spread at the lowest usable input amplification gain at a given DEPFET gate potential $V_{\text {gate-on }}$. The DCD input amplification gain is chosen such that the effective dynamic input range can accommodate the remaining spread of the compressed pedestal distribution. The smallest gain is selected where the full pedestal distribution and an adequate signal headroom are covered. The dependence of the pedestal and noise distribution on the DCD input gain is shown in Appendix B. For module W11_OF2, at a gate potential of $V_{\text {gate-on }}=-1.65 \mathrm{~V}$, a gain of $96 \mathrm{nA} / \mathrm{ADU}$ is achievable. The effective pedestal spread is measured as the full width at half maximum (FWHM) of the residual pedestal value distribution using the DHP raw data read-out mode. The distribution should be centred in the lower half of the dynamic range to provide sufficient signal headroom. The average of the pedestal distribution should thus ideally be $<125$ ADU. For the example module W11_OF2, the pedestal map and distribution is shown in Figure 7.5 with a pedestal spread FWHM of $109 \mathrm{ADU}$ at an average pedestal value of $127.8 \mathrm{ADU}$. The overall read-out noise is estimated as the average of the pixel-specific read-out noise distribution. An average noise value of $0.8 \mathrm{ADU}$ is measured for module W11_OF2. The pixel-specific noise distribution is shown in Figure 7.6. The DCD's analogue common mode compensation (ACMC) usually slightly improves the pedestal spread and the measured noise. For very few modules, this improvement is not observed and the ACMC is not used. The origin could be a defects in the DCD chips or an unfavourable interplay of specific DCD configurations.

Production failures can lead to certain anomalies in the pedestal and noise distribu- 


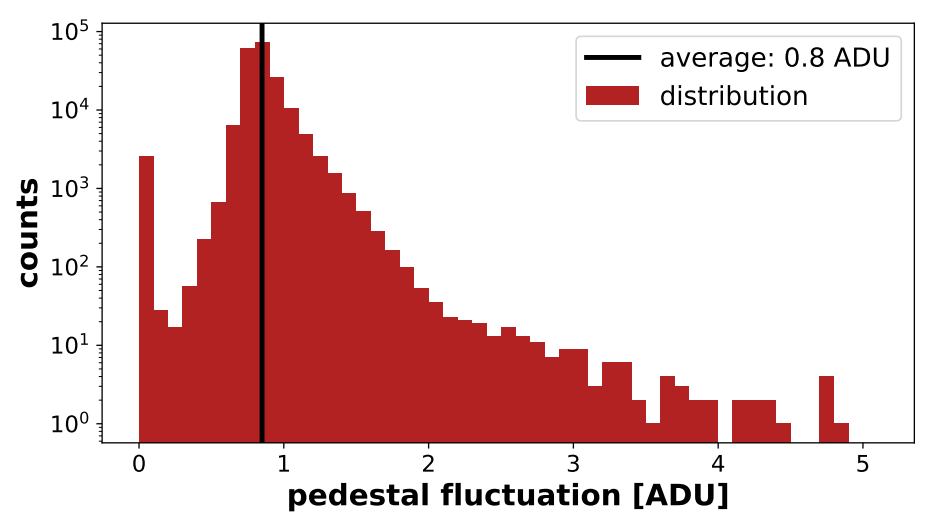

Figure 7.6.: Distribution of the fluctuations of the pedestal values of the $250 \times 768$ DEPFET pixels of module W11_OF2 used for quantifying the read-out noise. The values are histogrammed in bins of $0.1 \mathrm{ADU}$ width. The lowest bin collects non-functional pixels.

tions. Since one ADC channel in the DCD connects the drains of 192 DEPFET cells, referred to as drain line, a missing or misplaced bump-bond at one of the inputs causes 192 pixels to exhibit zero input current and thus zero pedestal value and noise. Slightly less critical is a discontinuation of the drain line somewhere on the matrix. In this case a fraction of the 192 pixels are lost. Due to the four-row read-out blocks, this manifests as a "silent" pixel every four pixels along a matrix column. Clearly, the example module W11_OF2 exhibits multiple problematic drain lines visible as a regular pattern of pixels along a column with low pedestal value in Figure 7.5. Similarly, a switcher output bump-bond can be missing or misplaced. This can result in a four-row of pixels never being switched on for read-out in case a $V_{\text {gate-on }}$ output is affected. This manifests in four consecutive rows of silent pixels. In case a clear potential is affected, a four-row of pixels is never cleared and collects charge until the internal gate is fully filled. This results in a four-row of pixels far outside of the DCD dynamic input range.

\subsubsection{Response to ionising radiation}

In the final step of the testing and characterisation procedure, the module is exposed to ionising radiation from a radioactive source. The module is illuminated from the top side and particles pass the metallisation layers before reaching the depleted bulk of the sensor. A $\beta$-source emitting electrons is thus best suited. Low-energy photons from a $\gamma$-source are absorbed to a significant fraction within the top side structures. The Göttingen test stand uses a Strontium-90 (Sr90) source which undergoes $\beta^{-}$-decay with a decay energy of $546.0 \mathrm{keV}$ [60]. As described in section 5.1, the expected energy deposition follows a Landau-shaped distribution. A simulation based on Geant4 [61] was conducted to obtain a reference energy deposition spectrum for a Sr90 source and a $75 \mu \mathrm{m}$ thick silicon material [62]. The top side structures or other influences are not 


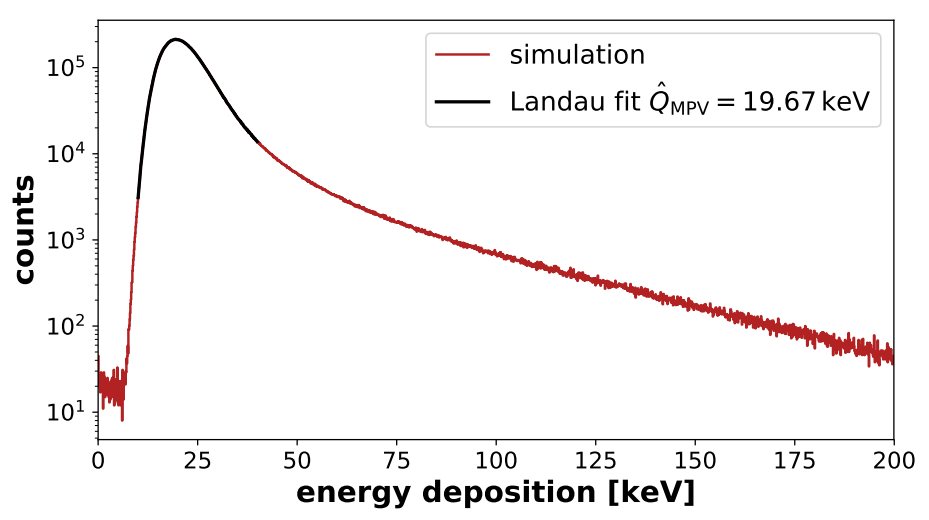

Figure 7.7.: Simulation of the distribution of the energy deposition of electrons from Sr90 $\beta$-decay in $75 \mu \mathrm{m}$ silicon [62]. Overall, $3 \times 10^{7}$ electrons were simulated and their energy deposition is histogrammed in bins of $0.1 \mathrm{keV}$ width. A Landau distribution is fitted to the histogram to obtain the most probable value (MPV). The uncertainty is estimated as half the bin width.

considered in the simulation. Figure 7.7 shows the reference spectrum with a most probable value (MPV) of the deposited charge $\hat{Q}_{\mathrm{MPV}}=19.67(5) \mathrm{keV}$ taken from a fit of a Landau distribution to the data in the range $10 \mathrm{keV}$ to $40 \mathrm{keV}$.

For the measurements, the Sr90 source is inserted into a holding structure and the outlet is positioned above the pixel matrix. Ideally, the whole sensor area is exposed to radiation, either by using a source with a large opening angle, or by automatically moving the source over the sensor. The latter was realised at the Göttingen test stand where the Sr90 source is moved in discrete steps of over the sensor area. During the measurements, the setup is shielded against light sources. Before positioning the source over the module, the reference values for the pedestal subtraction in the DHP are updated. While the source is moved over the sensor area, a uniform DHE-internal trigger is generated at $1 \mathrm{kHz}$ to $5 \mathrm{kHz}$. Data is recorded to disk by the DAQ software. The exposure time is chosen such that each pixel is estimated to collect a sufficient amount $(\mathcal{O}(100))$ of particle hits. This depends on the actual trigger rate, the activity of the source and its distance to the sensor. The zero-suppression threshold is set to $7 \mathrm{ADU}$.

The biasing potentials of the DEPFET pixel matrix can and need to be fine-tuned from module to module to yield an optimal sensor response. The procedure for finding the optimal sensor biasing scenario is explained in the following section. It is based on the tools used for characterising the sensor response that are presented in this section. Results presented here for module W11_OF2 are already based on the optimised biasing of $V_{\mathrm{HV}}=-60 \mathrm{~V}, V_{\mathrm{drift}}=-6 \mathrm{~V}$ and $V_{\text {clear-of }}=3 \mathrm{~V}$ and all remaining potentials as given in Table 6.1.

The recorded data is analysed to extract the measured energy deposition spectrum. Since the deposited charge can be spread over multiple pixels, the hit data is clustered first. Hits with signals above the zero-suppression threshold sharing a common edge 


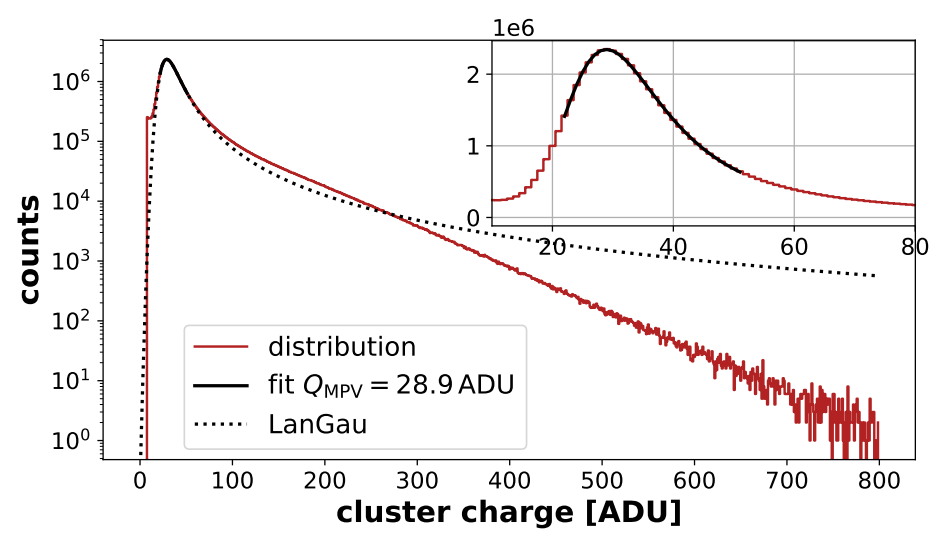

Figure 7.8.: Cluster charge distribution measured with a Sr90 source for module W11_OF2. The cluster charge values are histogrammed in bins of $1 \mathrm{ADU}$ width. A Gaussian folded Landau (LanGau) distribution is fitted to the data in the peak region (solid line). The dotted line indicates the LanGau distribution with the fitted parameters over the full range.

or corner are collected in a pixel cluster. The total cluster size is the number of pixels collected in the cluster. For module W11_OF2, an average cluster size of 1.6 was measured, where $95 \%$ of all clusters have a size of 4 or less. The cluster charge is the sum of the individual charges measured by the pixels belonging to the cluster. The cluster seed is referring to the pixel with the highest charge signal in the cluster. The cluster charges, given in units of ADU, are histogrammed to obtain a cluster charge distribution. It is expected to resemble a Landau distribution with Gaussian read-out noise folded in, referred to as LanGau. Figure 7.8 shows the cluster charge distribution of module W11_OF2. A LanGau function is fitted to the distribution in the peak region to extract an MPV of $Q_{\mathrm{MPV}}=28.9(5) \mathrm{ADU}$, where the uncertainty is estimated as half the bin width of $1 \mathrm{ADU}$. The peak region is defined as the data points within the 10th percentile $\left(P_{10}\right)$ and the 75 th percentile $\left(P_{75}\right)$ over the data. Due to the limited dynamic input range of the digitisation, the measured distribution is expected to be skewed in the high energy tail compared to an ideal Landau distribution. The discrepancy in the tail is confirmed by showing the LanGau over the full cluster charge range with the fitted parameters in Figure 7.8.

A calibration factor $k$, in units of $\mathrm{keV} / \mathrm{ADU}$, can be computed as the ratio $\hat{Q}_{\mathrm{MPV}} / Q_{\mathrm{MPV}}$ of the simulated MPV over the measured MPV and is used for converting pixel charge and cluster charge values from ADU to $\mathrm{keV}$. For the given module W11_OF2, the calibration factor is computed to $k=0.67(1) \mathrm{keV} / \mathrm{ADU}$, where the uncertainty is given by propagation of uncertainties of $\hat{Q}_{\mathrm{MPV}}$ and $Q_{\mathrm{MPV}}$. Charge values can be converted into number of electrons collected in the internal gate by dividing the value in $\mathrm{keV}$ by $\Delta E_{e h}=$ $3.6 \mathrm{eV}$, the electron-hole creation energy in silicon. The most probable cluster charge thus translates to 19.4(4) keV or 5400(111) electrons. The applied zero-suppression threshold translates to 1300(21) electrons and the average noise is 149(2) electrons. The observed 


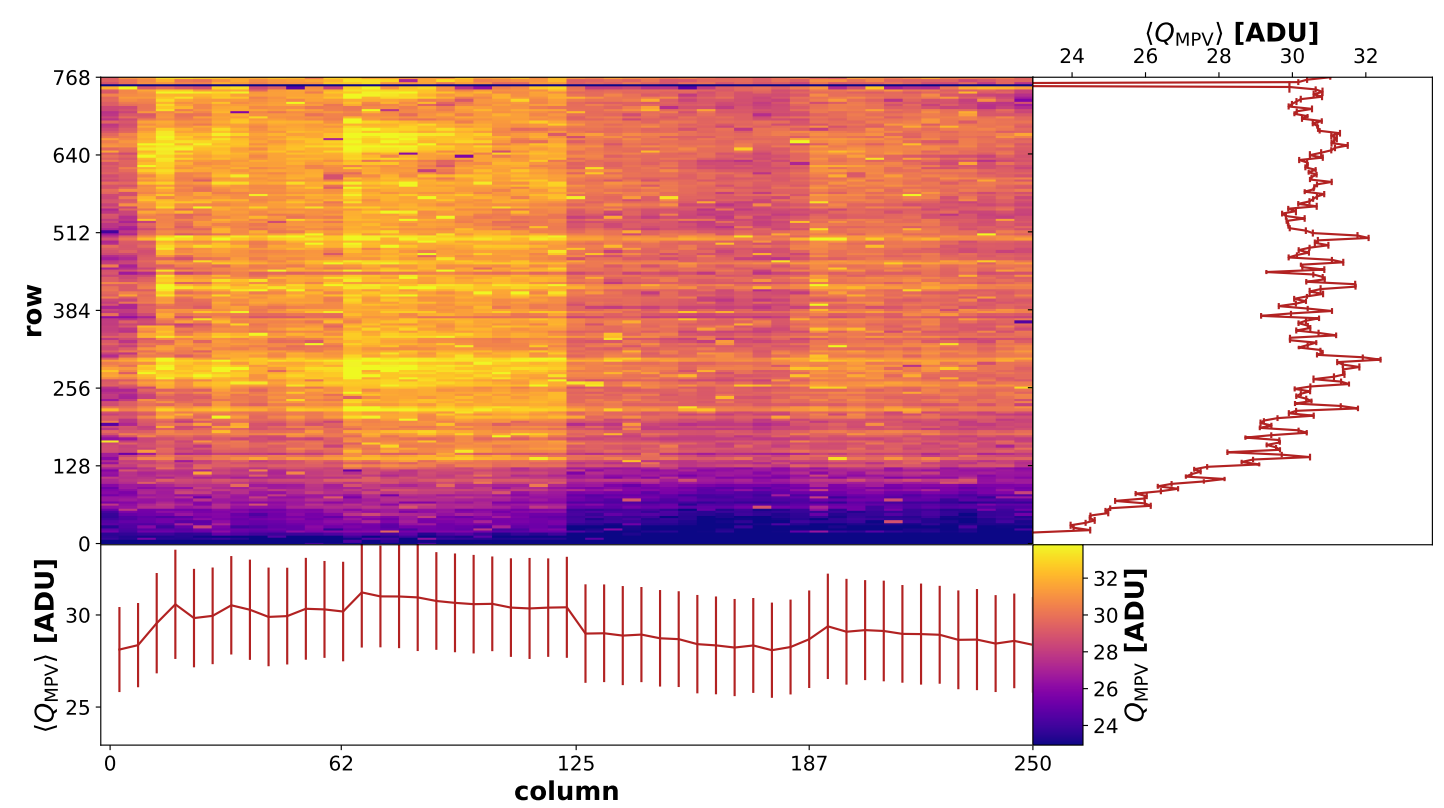

Figure 7.9.: Cluster charge MPV as a function of the column and row coordinate of the $250 \times 768$ DEPFET pixels of module W11_OF2. The projections in column and row display the average along columns and rows, respectively. The errorbars indicate the standard deviation along columns and rows, respectively.

noise is in good agreement with previous measurements [45]. Using the information on the DCD input amplification gain $g_{\mathrm{DCD}}$, the in-pixel amplification factor $g_{q}$ can be estimated. For the considered module, operated at a DCD gain of $g_{\mathrm{DCD}}=96 \mathrm{nA} / \mathrm{ADU}$, an in-pixel amplification of about $g_{q}=520(5) \mathrm{pA} /$ electron is computed.

The uniformity of the cluster charge MPV, and thus of the calibration factor and the in-pixel amplification, is investigated by considering the value of $Q_{\mathrm{MPV}}$ as a function of the pixel column and row coordinate. To ensure a reliable fit of the LanGau distribution for extracting the MPV, pixels are collected in super-pixel bins of size $u \times v=5 \times 4$. Figure 7.9 shows the cluster charge MPV for each super-pixel bin for module W11_OF2. The projections on columns and rows show a gradient. The gradient, determined as $\left(P_{95}-P_{5}\right) /\left\langle Q_{\mathrm{MPV}}\right\rangle$, is in the order of $30 \%$, where pixels closer to the read-out ASICs (bottom) have a lower MPV than pixels on the far end of the module. A problematic pixel four-row (rows 752 to 755 ) yielding no signals is revealed close to the top. The observed non-uniformity in $Q_{\mathrm{MPV}}$ is taken into account by determining the calibration factor $k$ and the in-pixel amplification $g_{q}$ separately for each pixel four-row, based on the cluster charge distribution of the $250 \times 4$ pixels in the four-row. Figure 7.10 shows the values for $k$ and $g_{q}$ as a function of the four-row coordinate. The gradient over the pixel matrix, most pronounced for rows 0 to 150 , is clearly visible. The overall average values are $\langle k\rangle=0.67 \mathrm{keV} / \mathrm{ADU}$ and $\left\langle g_{q}\right\rangle=522 \mathrm{pA} /$ electron, respectively. The per four-row 


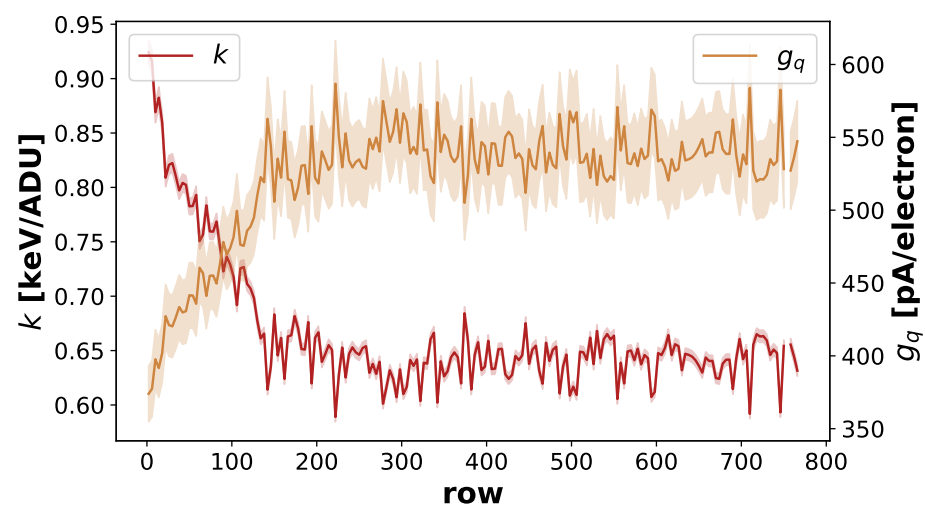

Figure 7.10.: Cluster charge calibration factor $k$ and estimated in-pixel amplification $g_{q}$ per four-row of module W11_OF2. The light coloured bands around the lines indicate the respective uncertainty. The problematic read-out block (rows 752 to 755 ) is excluded.

values for $k$ are used for a position dependent conversion of cluster charges to cluster energies. The resulting cluster energy spectrum is shown in Figure 7.11. The LanGau fit yields an MPV of 19.1(2) keV, with the uncertainty given by half the bin width. Considering the additional contribution by the uncertainty of the calibration factors, translating to about $0.3 \mathrm{keV}$, the obtained MPV is in agreement with the simulation (cf. Fig. 7.7).

A common figure of merit for assessing the performance of a detector is the signalto-noise ratio (SNR). A high SNR allows for a good separation of actual signal events from random and fake noise events. For the pixel detector modules, an effective read-out noise per pixel is available. A cluster-specific SNR can be defined as the ratio of the total cluster charge over the quadratic sum of the noise values of the pixels associated to the cluster. It is practical to assume, that the noise values of neighbouring pixels do not vary significantly and identify all pixel noise values with the noise of the seed pixel of the cluster. Thus, the cluster SNR is

$$
\mathrm{SNR}=\frac{S}{\sqrt{d} N},
$$

where $S$ is the total cluster charge, $N$ the noise of the seed pixel and $d$ the number of pixels associated to the cluster. The distribution of the cluster SNR values for module W11_OF2 is shown in Figure 7.12. It resembles a Landau distribution. A fit of a LanGau function yields an MPV of 31.39(5), where the uncertainty is given by half the bin width. Overall, $95 \%$ of all clusters exhibit an SNR value of $P_{5}=18.1$ or larger. The average SNR is 43.7 , while a simple estimate as the overall cluster charge $Q_{\mathrm{MPV}}$ over averaged noise yields an SNR value of 36.1. The observed SNR values are in good agreement with expectations from prototype measurements [45].

The response of the module to the ionising radiation is required to yield an MPV of 


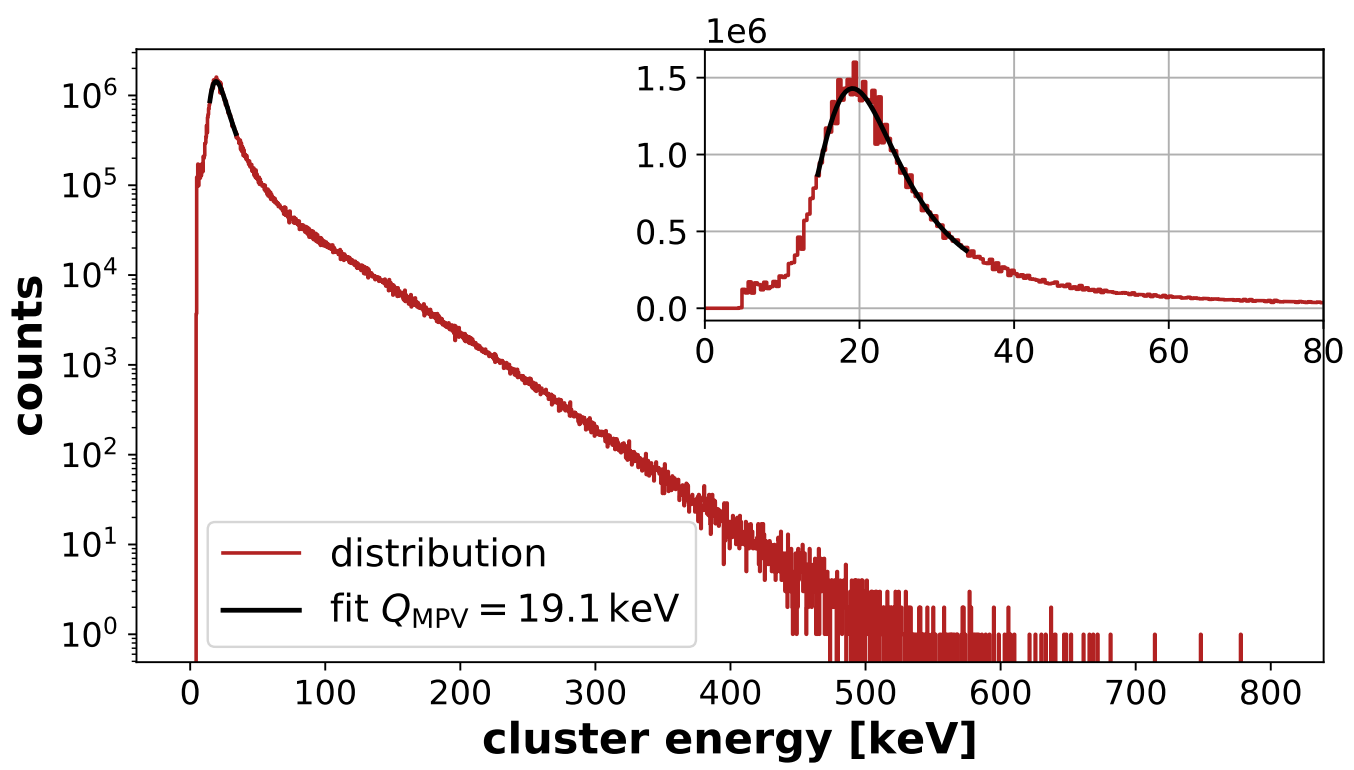

Figure 7.11.: Calibrated cluster energy distribution (Sr90 source) for module W11_OF2. The cluster charge values are histogrammed in bins of $0.4 \mathrm{keV}$ width. A LanGau distribution is fitted to the data in the peak region (solid line).

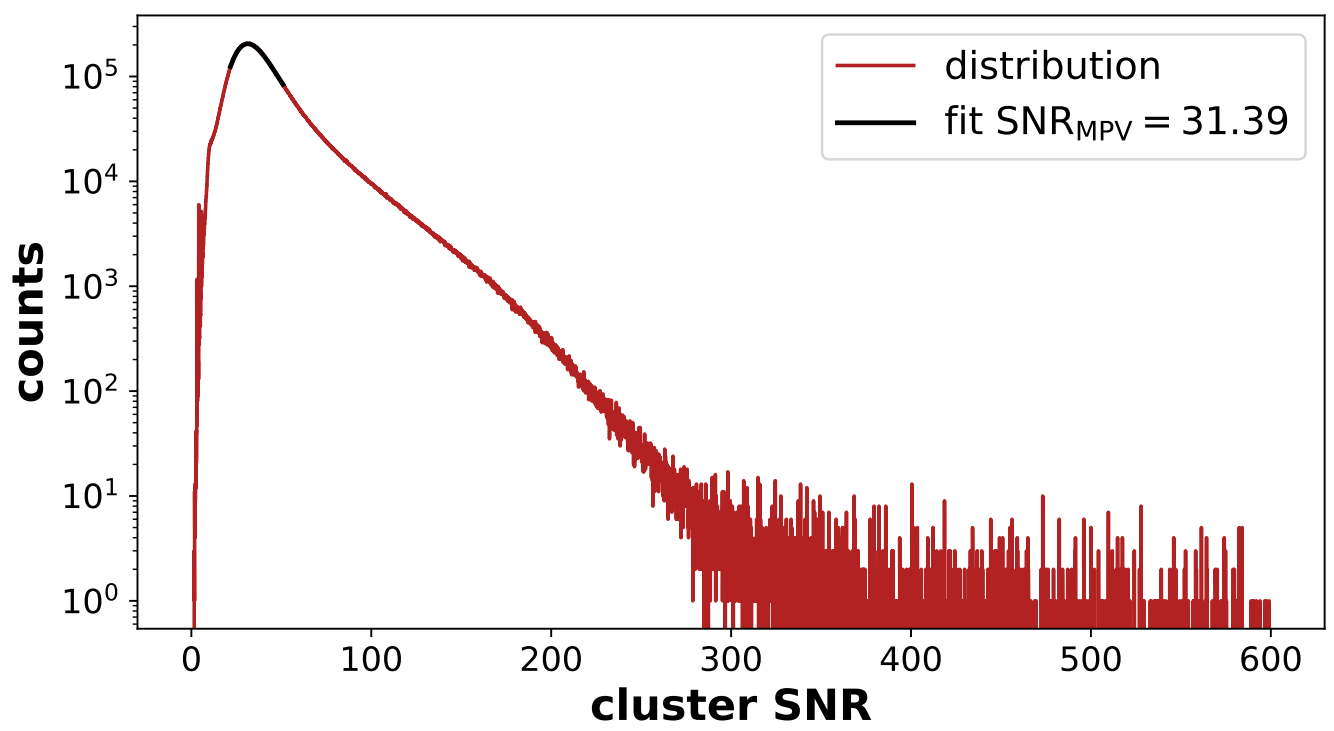

Figure 7.12.: Distribution of the cluster signal-to-noise ratio (SNR) for module W11_OF2. The values are histogrammed in bins of 0.1 width. A LanGau distribution is fitted to the data in the peak region (solid line). 
the cluster SNR distribution $\mathrm{SNR}_{\mathrm{MPV}} \gtrsim 20$ and an extracted in-pixel amplification of $g_{q} \gtrsim 400 \mathrm{pA} /$ electron to pass the test. A final grade is assigned to each module based on the fraction of non-functional (dead) pixels $f_{\dagger}$. A pixel is considered non-functional if the hit count over the full exposure time is equal to zero. This comprises for example open drain lines (cf. Fig. 7.5) or "silent" rows (cf. Fig. 7.9). For module W11_OF2, the fraction of non-functional pixels is $1.8 \%$. The grade $\mathrm{A}$ is assigned if the fraction of nonfunctional pixels is $f_{\dagger} \leq 1 \%$, that means $\geq 99 \%$ of the DEPFET pixels are functional. The grade $\mathrm{B}$ is assigned if the fraction of non-functional pixels is $1 \% \leq f_{\dagger} \leq 5 \%$. If a module exhibits a larger fraction of non-functional pixels, $f_{\dagger} \geq 5 \%$, the grade $\mathrm{F}$ is assigned and the module should not be considered for installation in Belle II. Module W11_OF2 hence is assigned the grade B.

\subsection{Optimisation of the DEPFET biasing}

With the tools for characterising the response to ionising radiation at hand, the task is the optimisation of the DEPFET potentials. The eleven potentials required for biasing, readout and clearing of the DEPFET cells (cf. Tab. 6.1) have complex cross-dependencies. They can be divided in the two domains charge collection and read-out/clear.

The read-out/clear potentials are $V_{\text {gate-on }}$ and $V_{\text {clear-on }}$. A four-row of the pixel matrix is read-out and cleared in a time window of $100 \mathrm{~ns}$ of the $20 \mu \mathrm{s}$ integration time of the full matrix. Thus, these potentials are active at most $0.5 \%$ of the time and have only a very minor influence on the potential landscape governing the collection of deposited charge into the internal gates of the DEPFET cells. As discussed, $V_{\text {gate-on }}$ directly influences the internal amplification $g_{q}$. An optimal $V_{\text {gate-on }}$ yields a convenient $g_{q}$ while maintaining, in conjunction with the DCD input amplification, a manageable pedestal spread. For unirradiated sensors, the optimal $V_{\text {gate-on }}$ is close to $-2 \mathrm{~V}$. The optimal clearing potential $V_{\text {clear-on }}=19 \mathrm{~V}$ provides an efficient and fast removal of charges from the internal gate.

The potentials of the charge collection domain determine the potential landscape during the collection of deposited charges. Simulations show, that the three potentials $V_{\mathrm{HV}}, V_{\text {drift }}$ and $V_{\text {clear-of }}$ dominantly impact the charge collection in the DEPFET cells. For this reason and to reduce the complexity of the optimisation procedure, all other potentials are kept at their standard parameters recommended by the sensor design as given in Table 6.1. The depletion voltage $V_{\mathrm{HV}}$ must be appropriately chosen to yield a fully depleted bulk. If the depletion voltage is too positive (closer to zero), parts of the silicon bulk are not depleted. Deposited charges in not depleted regions are not separated and the corresponding electrons are not eventually collected in the internal gate. Thus, a fraction of the charge deposition is lost. This scenario is referred to as under-depletion. On the other hand, a DEPFET sensor also knows the case of over-depletion. For a too negative $V_{\mathrm{HV}}$, the drift paths of deposited charges are pushed towards the drift and transistor implants on the top side. A fraction of the charge can then be lost in these structures before reaching the internal gate by lateral drift. Especially the charge loss in the over-depletion scenario depends strongly on the drift potential $V_{\text {drift }}$ and the clear 


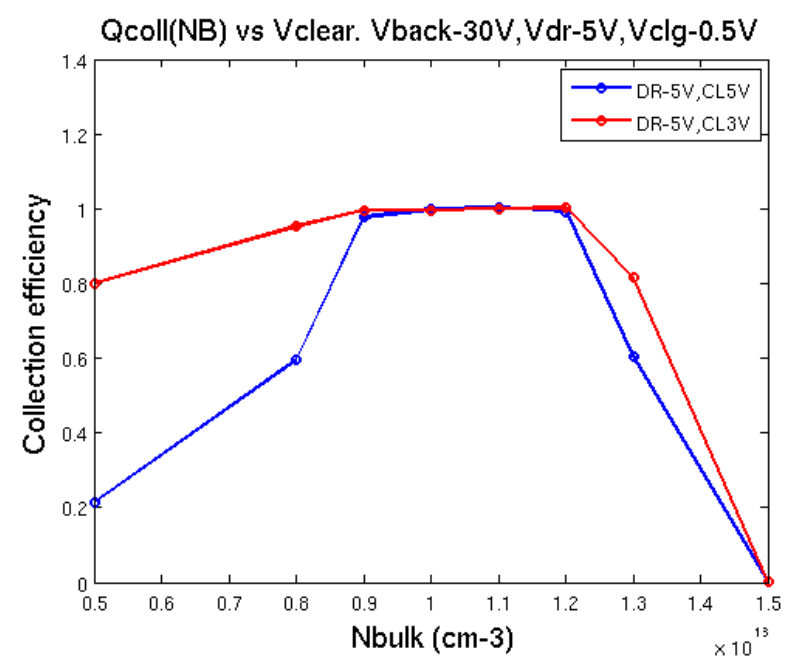

Figure 7.13.: Simulation of the charge collection efficiency as a function of the bulk depletion for two biasing scenarios. The simulations were conducted by Rainer Richter from the Halbleiterlabor of the Max-Planck-Society [53].

shielding potential $V_{\text {clear-off }}$. Figure 7.13 shows a simulation of the charge collection efficiency dependent on the bulk depletion for values of $V_{\text {clear-off }}=5 \mathrm{~V}$ and $V_{\text {clear-off }}=$ $3 \mathrm{~V}$ at a drift potential of $V_{\text {drift }}=-5 \mathrm{~V}$. Technically, it shows the collection efficiency as a function of the bulk doping concentration at a fixed $V_{\mathrm{HV}}$, which is equivalent to a variation of $V_{\mathrm{HV}}$ at a fixed doping concentration.

Detailed measurements of the influence of the biasing potentials $V_{\mathrm{HV}}, V_{\mathrm{drift}}$ and $V_{\text {clear-off }}$ on the charge collection were conducted on selected modules. In depth scans of various biasing scenarios for each module in the series testing are not feasible due to a very tight schedule. The measurements discussed in the following take a few days per module, dependent of course on the activity of the employed radioactive source and the geometrical arrangement of the source and the module. A feasible optimisation strategy is proposed based on the results of these investigations.

\subsubsection{Over-depletion and under-depletion}

Radioactive source measurements in the laboratory do not provide an absolute measure of the charge collection efficiency. Instead, the influence of the biasing potentials on the measured cluster charge distribution is investigated. This is effectively an approximation of relative changes to the charge collection efficiency. To be sensitive to variations across the sensor area, the cluster charge MPV, $Q_{\mathrm{MPV}}$, is determined per super-pixel of size $u \times v=10 \times 8$ by fitting of a LanGau distribution. Figure 7.14 depicts the averaged value of $Q_{\mathrm{MPV}}$ (and percentiles) over the super-pixels as a function of $V_{\mathrm{HV}}$. The observed behaviour agrees with the expectations from simulations (cf. Fig. 7.13). The underdepletion regime $\left(V_{\mathrm{HV}} \gtrsim-53 \mathrm{~V}\right)$ and the over-depletion regime $\left(V_{\mathrm{HV}} \lesssim-63 \mathrm{~V}\right)$ are clearly visible. In between, there is a plateau over a range of about $5 \mathrm{~V}$ to $10 \mathrm{~V}$. It is 


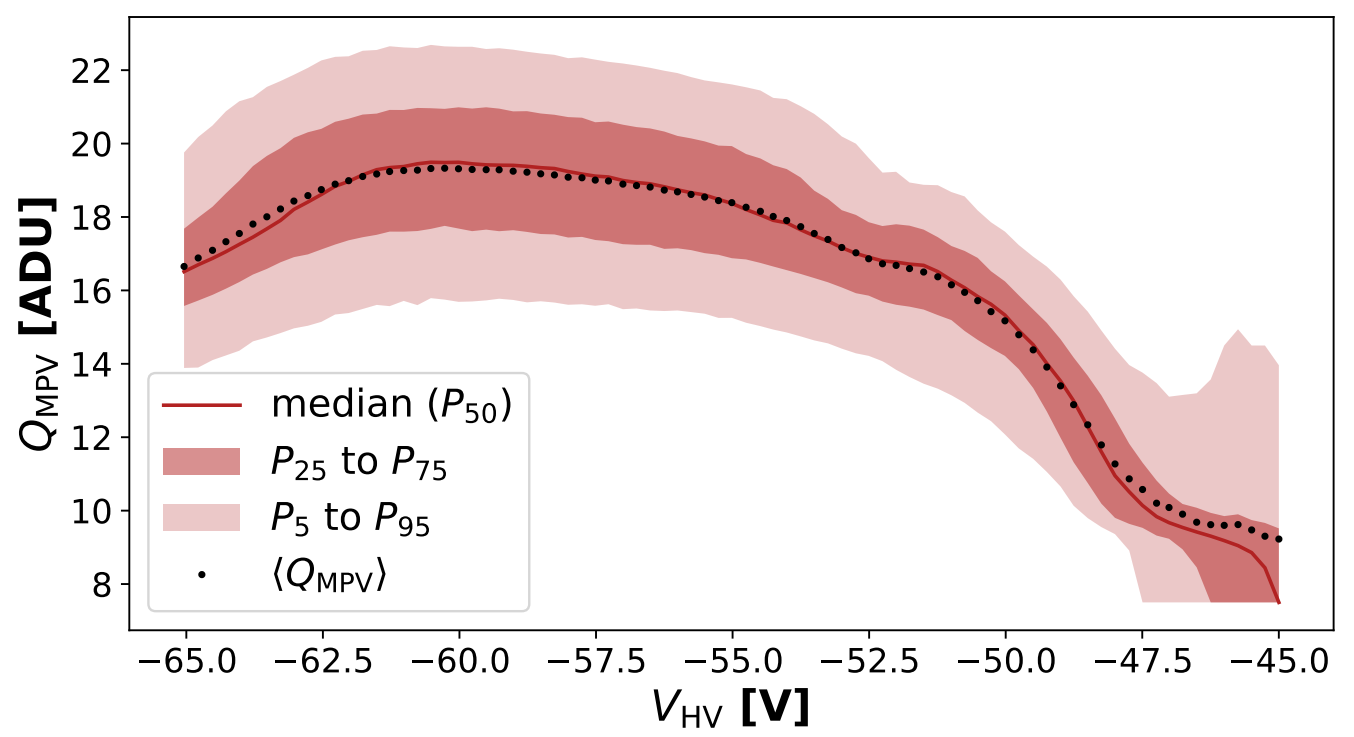

Figure 7.14.: Distribution of the cluster charge MPV per super-pixel as a function of the depletion voltage for $V_{\text {drift }}=-5 \mathrm{~V}$ and $V_{\text {clear-off }}=5 \mathrm{~V}$ measured on module W40_IF.

interesting to examine the actual spatial distribution of the $Q_{\mathrm{MPV}}$ values as a function of the super-pixel coordinates for selected values of the depletion voltage. Figure 7.15 displays the spatial $Q_{\mathrm{MPV}}$ distribution for a value of $V_{\mathrm{HV}}$ of the over-depletion, the plateau and the under-depletion regime, respectively. Despite the already discussed linear gradient over the pixel matrix, ring-like structure are visible. This continuous, non-linear dependence of $Q_{\mathrm{MPV}}$ on the position on the sensor cannot be explained by features of the read-out ASICs or the pixellation of the sensor area. Instead, the origin must be a property of the bulk of the sensor. It is observed, that areas of the sensor that still exhibit a sufficiently large, "plateau-like" $Q_{\mathrm{MPV}}$ in the over-depletion regime, reveal a particularly low $Q_{\mathrm{MPV}}$ in the under-depletion regime and vice versa. This suggests that over-depletion and under-depletion set in at different $V_{\mathrm{HV}}$ for different areas of the sensor. Two areas of the sensor, belonging to district "ring regions", are selected and their $V_{\mathrm{HV}}$ dependence is compared in Figure 7.16. The two curves are shifted with respect to each other. The relative offset in $Q_{\mathrm{MPV}}$ is due to the linear gradient over the sensor. At a certain depletion voltage, parts of the sensor are already under-depleted (or over-depleted), while other parts are still in their respective plateau region. This effect has already been observed in the high-ohmic float zone silicon wafer material used for DEPFET sensors in [63] and is explained by variations of the bulk doping concentration across the sensor area. These variations are observed on all pixel detector modules when a non-optimal biasing scenario in the over-depletion or under-depletion region is applied. The task of the biasing optimisation is to find a biasing scenario where all parts of the sensor are fully depleted and the overlap of plateau regions of all sensor areas result in 

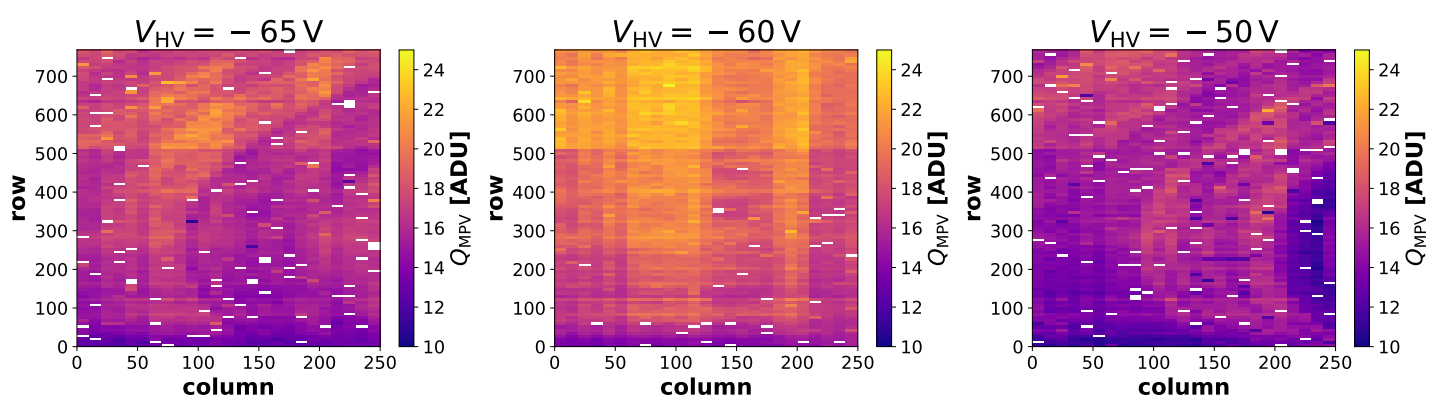

Figure 7.15.: Spatial distribution of the cluster charge MPV per super-pixel for three different depletion voltages. The other biasing potentials are $V_{\text {drift }}=-5 \mathrm{~V}$ and $V_{\text {clear-off }}=5 \mathrm{~V}$ measured on module W40_IF. For some super-pixels, the LanGau fit does not yield a reliable value of $Q_{\mathrm{MPV}}$ (white spots).
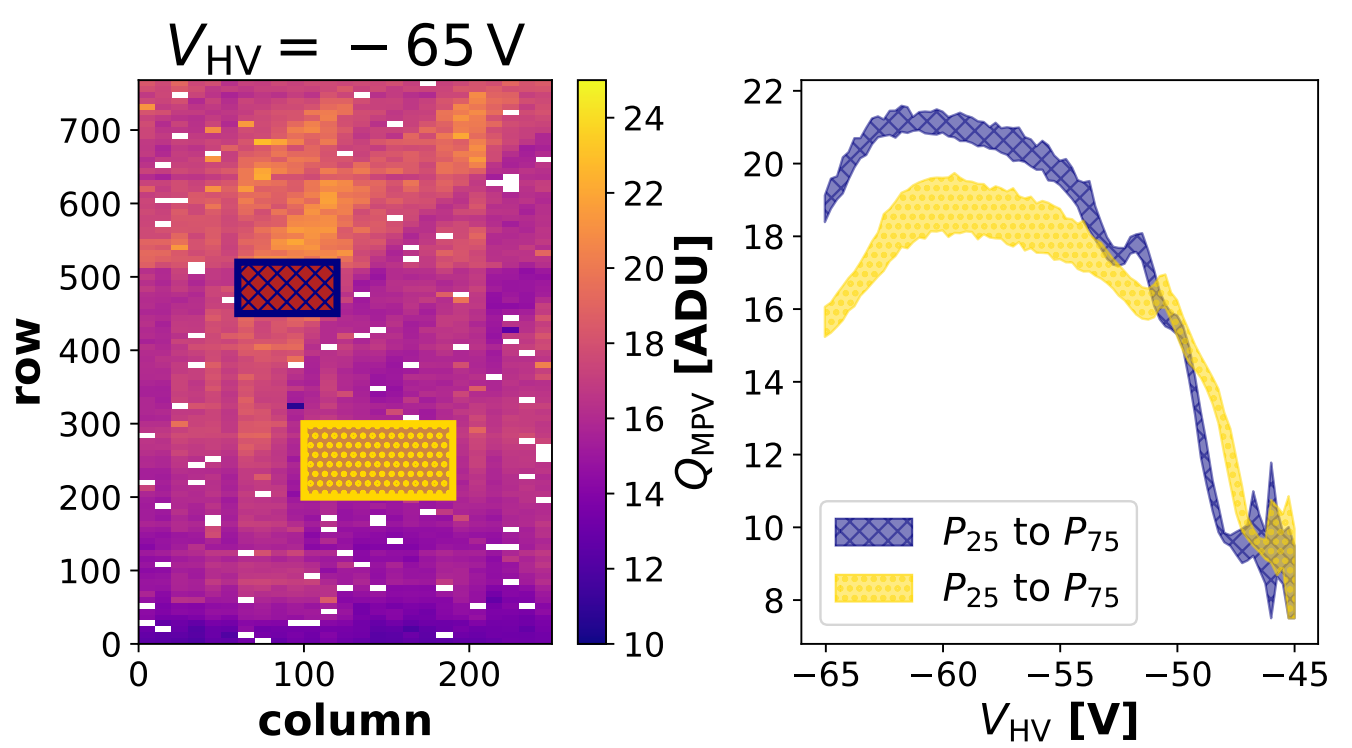

Figure 7.16.: Dependence of $Q_{\mathrm{MPV}}$ on the depletion voltage for two areas on the sensor associated to different "ring-regions". The other biasing potentials are $V_{\text {drift }}=-5 \mathrm{~V}$ and $V_{\text {clear }- \text { off }}=5 \mathrm{~V}$ measured on module W40_IF. 


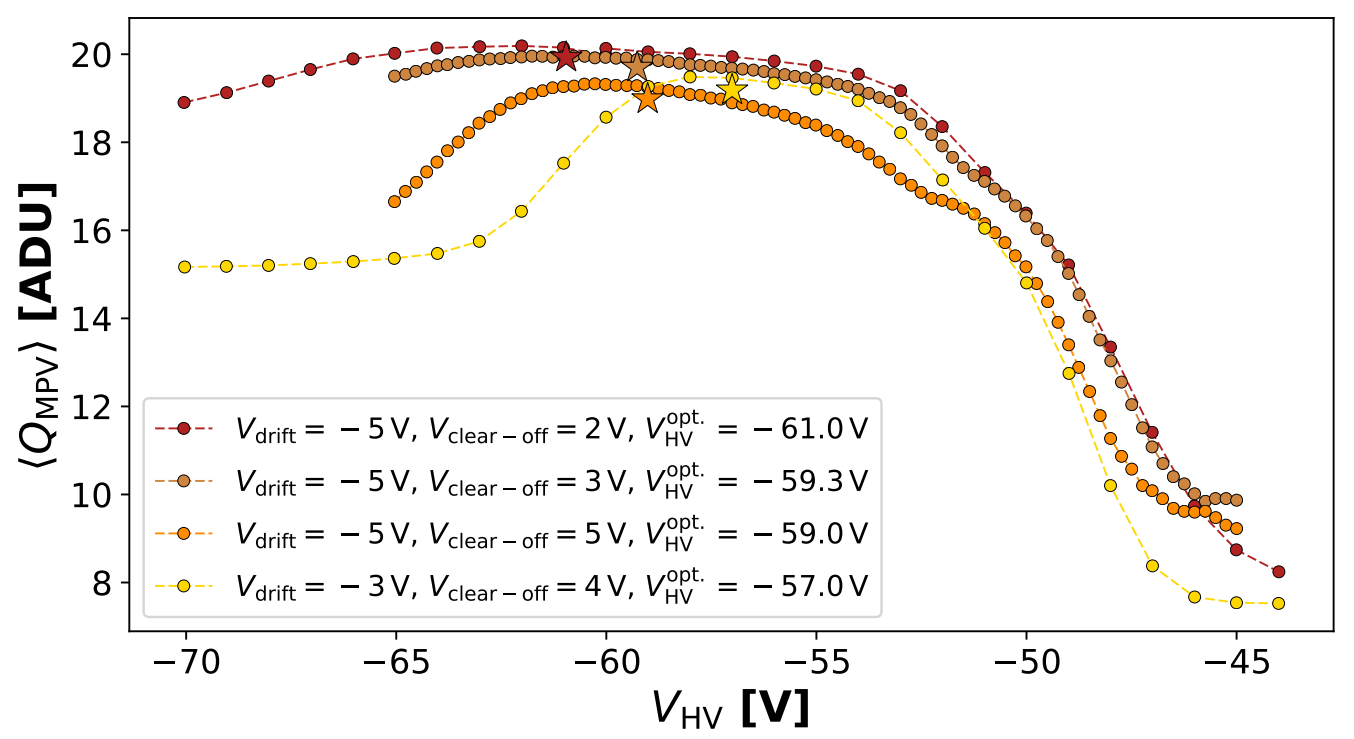

Figure 7.17.: Depletion voltage dependence of the average $Q_{\mathrm{MPV}}$ over all super-pixel for different values of the drift and clear shielding potential, measured on module W40_IF. The star marker indicates the respective extracted optimum of $V_{\mathrm{HV}}$ as described in the text, the values are given in the legend.

an effective plateau region with a convenient width.

\subsubsection{Drift and clear shielding optimisation}

The simulations suggest that a different choice for the drift potential, $V_{\text {drift }}$ and the clear shielding potential $V_{\text {clear-off }}$ can yield a wider plateau region (cf. Fig. 7.13). The depletion voltage dependence for different values of $V_{\text {drift }}$ and $V_{\text {clear-off }}$ is compared in Figure 7.17. Indeed, a much wider plateau region is observed when decreasing the clear shielding potential $V_{\text {clear-off }}=5 \mathrm{~V} \rightarrow 2 \mathrm{~V}$ at a drift potential of $V_{\text {drift }}=-5 \mathrm{~V}$. Moreover, the overall average of the cluster charge MPV seems to slightly increase as well. While the under-depletion region is rather consistent among the different biasing scenarios, the over-depletion region is significantly reduced by a decreased $V_{\text {clear-off }}$. This is consistent with the expectation from the simulation, since in the under-depletion regime charges are lost in undepleted regions of the bulk, while in the over-depletion case, the top-side structures and potentials are most relevant. Similar measurements on other modules confirm this behaviour as well as seen in Figure 7.18. For reaching a wide and maximised (regarding the value of $\left\langle Q_{\mathrm{MPV}}\right\rangle$ ) plateau region, a drift potential of $V_{\text {drift }} \leq-4 \mathrm{~V}$ is preferred consistently across different modules. The clear shielding potential at the same time should not exceed a value of $V_{\text {clear-off }} \leq 4 \mathrm{~V}$. A lower limit of $V_{\text {clear-off }} \geq 1 \mathrm{~V}$ is given by the possibility of back-injection of charges from the clear electrode below this threshold [64]. 
7. Series Testing of Pixel Detector Modules for Belle II

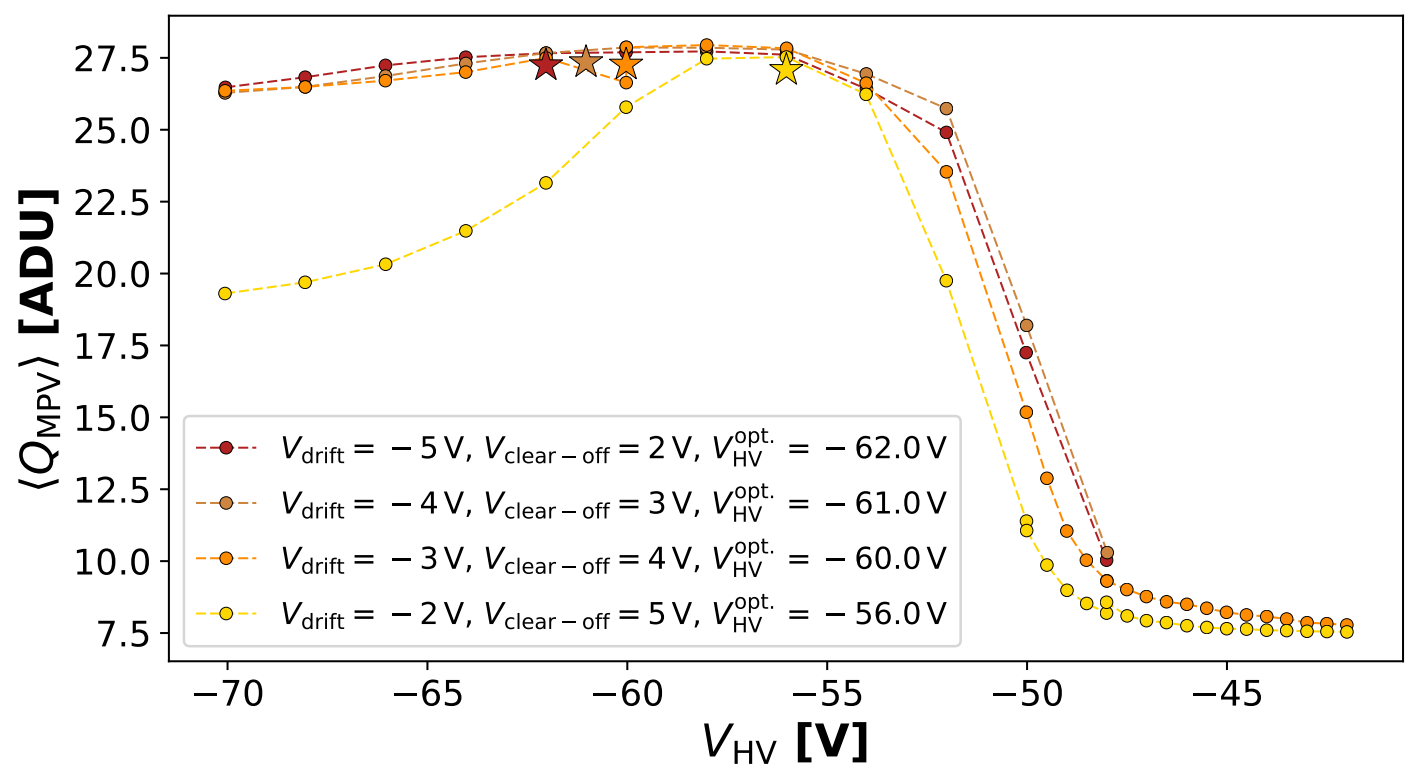

(a) measured on module W03_OB1

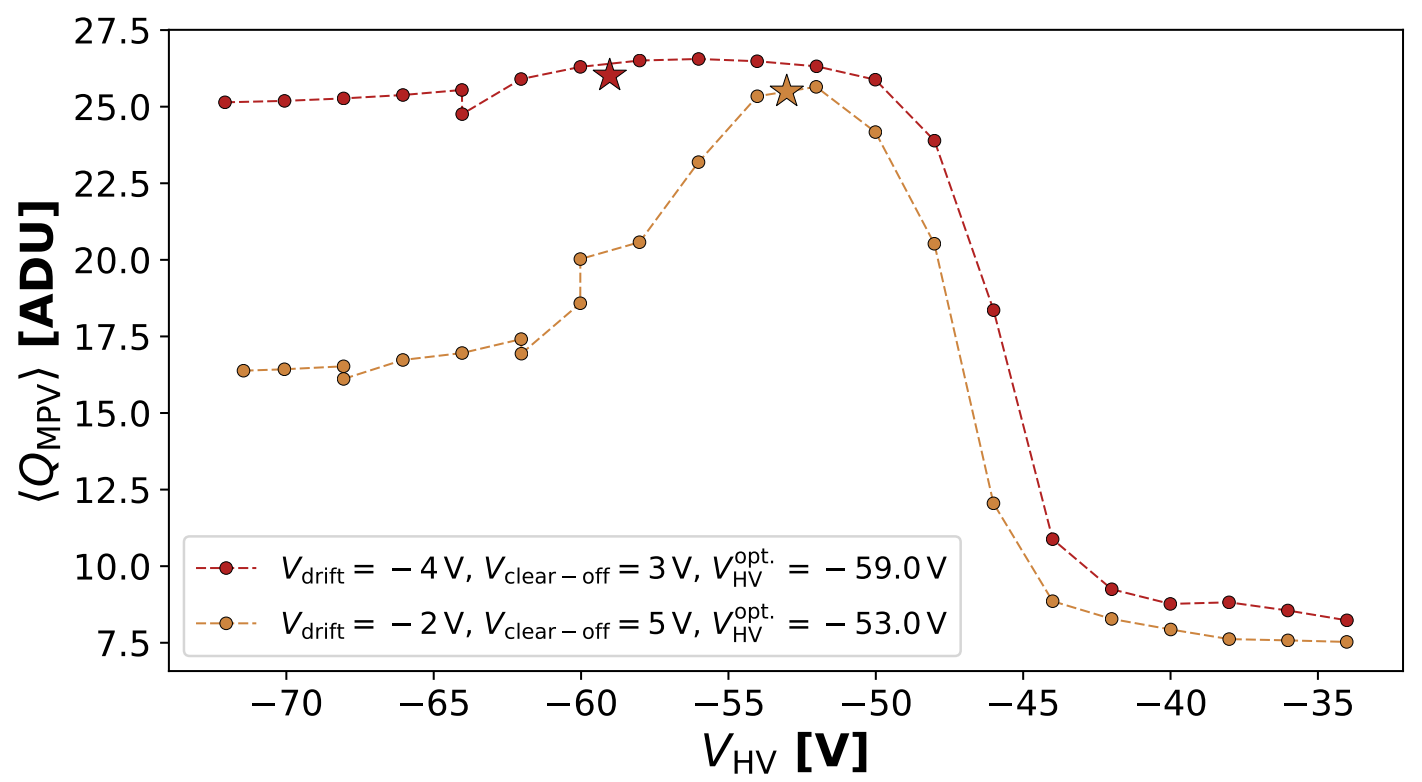

(b) measured on module W05_OB1

Figure 7.18.: Depletion voltage dependence of the average $Q_{\mathrm{MPV}}$ over all super-pixel for different values of the drift and clear shielding potential. The star marker indicates the respective extracted optimum of $V_{\mathrm{HV}}$ as described in the text, the values are given in the legend. 


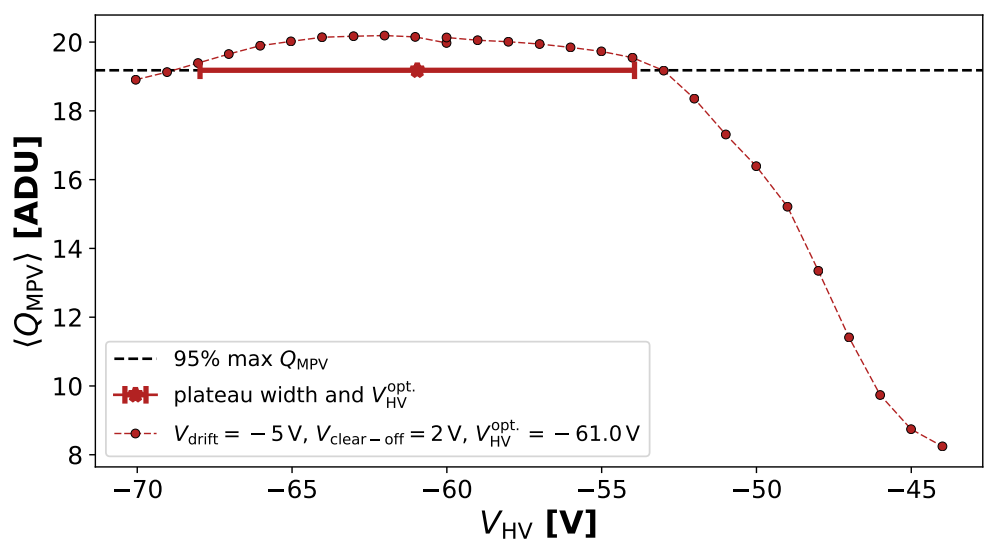

Figure 7.19.: Definition of the plateau width and finding of the optimal depletion voltage for a specific biasing scenario measured on module W40_IF.

\begin{tabular}{lcccc}
\hline module & $V_{\text {drift }}[\mathrm{V}]$ & $V_{\text {clear-off }}[\mathrm{V}]$ & $V_{\mathrm{HV}}^{\text {opt. }}[\mathrm{V}]$ & plateau width $[\mathrm{V}]$ \\
\hline W03_OB1 & -5 & 2 & -62.0 & 16.0 \\
W03_OB1 & -4 & 3 & -61.0 & 14.0 \\
W03_OB1 & -3 & 4 & -60.0 & 12.0 \\
W03_OB1 & -2 & 5 & -56.0 & 4.0 \\
\hline W05_OB1 & -4 & 3 & -59.0 & 18.0 \\
W05_OB1 & -2 & 5 & -53.0 & 2.0 \\
\hline W40_IF & -5 & 2 & -61.0 & 14.0 \\
W40_IF & -5 & 3 & -59.3 & 11.5 \\
W40_IF & -5 & 5 & -59.0 & 8.0 \\
W40_IF & -3 & 4 & -57.0 & 6.0 \\
\hline
\end{tabular}

Table 7.1.: Summary of the evaluation of different biasing scenarios.

The optimal biasing scenario is determined by maximising the width of the plateau region. The plateau width is defined as the range of measured depletion voltages with a corresponding average $Q_{\mathrm{MPV}}$ that is above a threshold of $95 \%$ of the maximum measured $Q_{\mathrm{MPV}}$ for the respective biasing scenario. The optimal depletion voltage, $V_{\mathrm{HV}}^{\text {opt. }}$, is then computed as the average of all $Q_{\mathrm{MPV}}$ above this threshold. Figure 7.19 illustrates

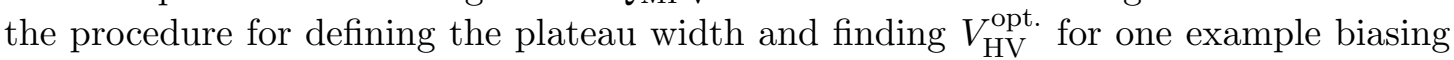
scenario. Table 7.19 summarises the plateau width and $V_{\mathrm{HV}}^{\text {opt. }}$ for the measured biasing scenarios and modules. This optimisation procedure consistently favours DEPFET biasing potentials of $V_{\text {drift }} \approx-5 \mathrm{~V}, V_{\text {clear-off }} \approx 2 \mathrm{~V}$ and $V_{\mathrm{HV}} \approx-60 \mathrm{~V}$. These findings constrain the reasonable parameter space for the biasing optimisation significantly. A coarse scan of biasing scenarios in the range $V_{\text {drift }}=-5 \pm 1 \mathrm{~V}, V_{\text {clear-off }}=3 \pm 1 \mathrm{~V}$ and $V_{\mathrm{HV}}$ from $-54 \mathrm{~V}$ to $-70 \mathrm{~V}$ in steps of $2 \mathrm{~V}$ should be sufficient to find a convenient plateau width. The optimal biasing scenario is chosen by maximising the plateau width. 


\begin{tabular}{llll}
\hline module & date & grade & comment \\
\hline W40_IF & Oct. 2017 & B & setup qualification module, investigated in beam tests \\
\hline W43_IB & Nov. 2017 & A & no biasing optimisation \\
W03_IB & Dec. 2017 & A & - \\
W06_OB1 & Jan. 2018 & B & no biasing optimisation \\
W12_OB2 & Feb. 2018 & B & - \\
W11_OF2 & Feb. 2018 & B & investigated in beam tests \\
W32_OF1 & Feb. 2018 & A & - \\
W46_OB1 & Feb. 2018 & A & - \\
W41_OF1 & Feb. 2018 & A & - \\
W09_OB2 & Feb. 2018 & A & - \\
W45_OF1 & Mar. 2018 & A & - \\
W05_OB1 & Mar. 2018 & - & high noise in ASIC pair 3, investigated in beam tests, \\
W03_OB1 & Mar. 2018 & B & investigated in irradiation tolerance measurement \\
W44_OB2 & Jun. 2018 & A & - \\
W04_OB1 & Jun. 2018 & - & high currents in DCD supply and failing JTAG chain \\
W43_OF1 & Jun. 2018 & A & - \\
W04_OF1 & Aug. 2018 & - & continues HV current increase during operation, \\
W05_OF1 & Jun. 2019 & A & tested up to ADC optimisation \\
\hline
\end{tabular}

Table 7.2.: List of modules characterised at the Göttingen test stand in chronological order. Given are the module label, the month of test, the final module grade and an optional comment. Details are discussed in the text.

\subsection{Results from the Göttingen test stand}

Over the course of several months, a total of 17 pixel detector modules were tested, characterised and graded at the Göttingen test stand, as listed in Table 7.2. The series testing started in the last quarter of 2017 with the setup qualification employing module W40_IF. Mostly outer layer modules (15 out of 17 ) were tested. The main part of the series testing was accomplished until mid of 2018. A final outer layer module, W05_OF1 was following mid of 2019. In summary, ten devices have been assigned the grade A, four devices were graded B. A number of three modules failed the testing and characterisation program at certain stages. Some distinctive features of selected modules are discussed in the following.

W40_IF (qualitification module) This was one of the first modules to be glued to a inner layer ladder. Since the paired IB-type module ceased operation after the gluing 


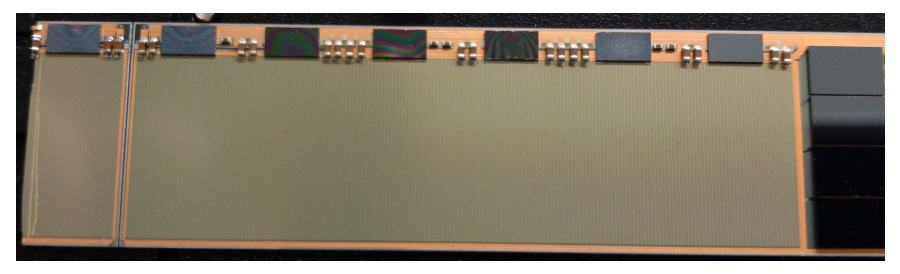

Figure 7.20.: Picture of the qualification module W40_IF with the remnant "IB module tail".

procedure $^{9}$, the ladder was mechanically cut to have the IF-type module available for testing purposes. Despite a small mechanical "IB module tail", the operation of the IF module is not significantly influenced. A picture of the module is shown in Figure 7.20. With its known characteristics, the module was selected to serve as a laboratory setup qualification reference. The correct functionality of the services (power supply, back-end DHH system, slow control) is confirmed when the characteristics of the qualification module can be reproduced at a given setup. The module was characterised in more detail in a beam test in 2018. The beam tests are described in Chapter 8 .

W43_IB Due to a very tight schedule of the series testing, several parts of the testing procedure were developed and improved with the first modules on the test stand. Especially the thorough investigation of the response to ionising irradiation via radioactive sources was not yet fully established for the first modules tested in Göttingen. The module W43_IB was only exposed to the Sr90 source in the area of the pixel rows 0 to 500. The results (notably the fraction of masked pixels) are thus restricted to this area. Moreover, only one biasing scenario was measured. The latter is also the case for module W06_OB1.

W05_OB1 This module exhibits a peculiar noisy read-out that is restricted to pixels read out by the third DCD-DHP AISC pair (columns 125 to 189). Figure 7.21 shows the pedestal fluctuation per pixel and the number of hits above threshold recorded during a radioactive source measurement. A pattern of "blocks" of high noise in the said columns is observed. The pattern is not fixed and changes between power cycles or when the module is mechanically manipulated. The origin of this effect is not understood. Due to the restriction in columns, it is either caused by an internal malfunction of the corresponding DCD or DHP ASIC, or it is a result of improper bump bonds between the ASICs and the module. In response to this observation, outputs of the third ASIC pairs are rejected for this module. Technically, this means that the output driver for the off-module data links of the third DHP ASIC is disabled. Except for the read-out issue, the module exhibits grade A characteristics. However, a final grade was not assigned since the origin of the issue is unclear.

\footnotetext{
${ }^{9}$ The cause for module failures during the initial glueing procedures has been identified and the proce-
} dure was improved in response. 


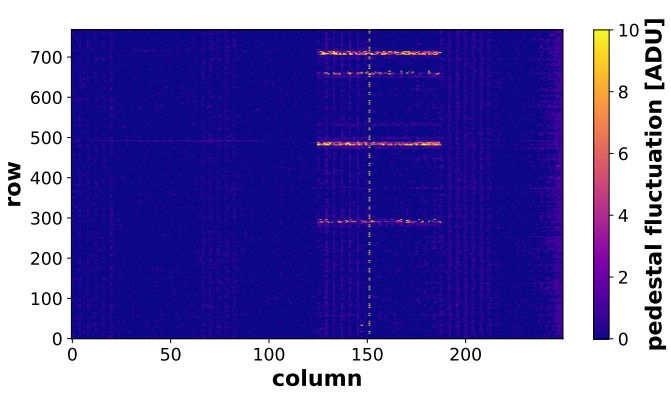

(a) pedestal noise

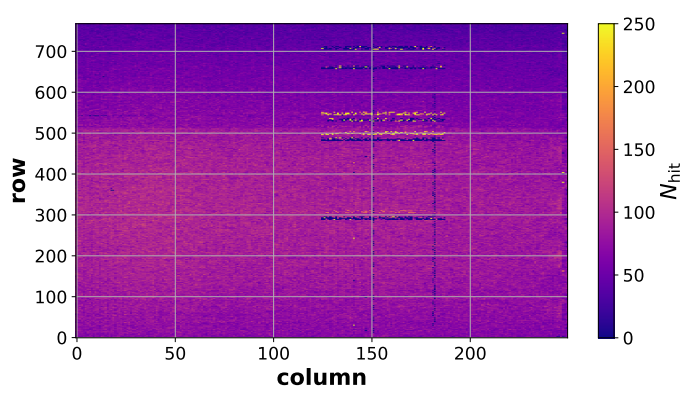

(b) collected hits

Figure 7.21.: Pedestal fluctuation and hit map of module W05_OB1. An irregular noise pattern is observed in columns 125 to 189 that cause a large number of fake hits. Due to changes of the pattern over time, a masking of only certain rows is not feasible.

The module W05_OB1 was selected to study the impact of X-ray irradiation on the performance of pixel detector modules. In a measurement campaign conducted in 2019 [48], the silicon oxide of the DEPFET gates received a total ionising dose of $266 \mathrm{kGy}$, while the estimated dose received by the relevant oxides in the ASICs was in the order of $15 \mathrm{kGy}$. For compensating the influence of accumulated stationary charges in the DEPFET silicon oxide, due to the ionising irradiation, the gate potentials have to be adjusted. It was shown in [48] that a stable operation of the module is still possible after irradiation without significant loss of performance. However, due to an operation mistake during the measurement campaign, the read-out of several four-rows were damaged by applying improper SWITCHER voltages. This increases the number of non-functional pixels of this module significantly.

W04_OB1 Already during the very first step of the series testing, this module failed the procedure. During the power-up, the module exhibits significantly higher currents in the DCD supply lines than considered safe for the ASIC. In addition, the ASIC configuration via JTAG was not possible. An X-ray inspection of the module at the HLL revealed an irregular connection between pads at the EOS introduced by the soldering of the Kapton interconnection. The module is set aside for a replacement of the Kapton.

W04_OF1 Successfully tested up to the ADC optimisation, this module exhibits a suspicious continues increase of the $V_{\mathrm{HV}}$ supply line current during radioactive source measurements. The current increase is problematic once the supply limit of the PSU for the $V_{\mathrm{HV}}$ channel is reached and, in reaction, the potential $V_{\mathrm{HV}}$ decreases. There is no mechanism proposed yet that could explain this behaviour. The module is set aside for detailed investigations.

The following figures and tables provide a summary of the characteristics of the mod- 


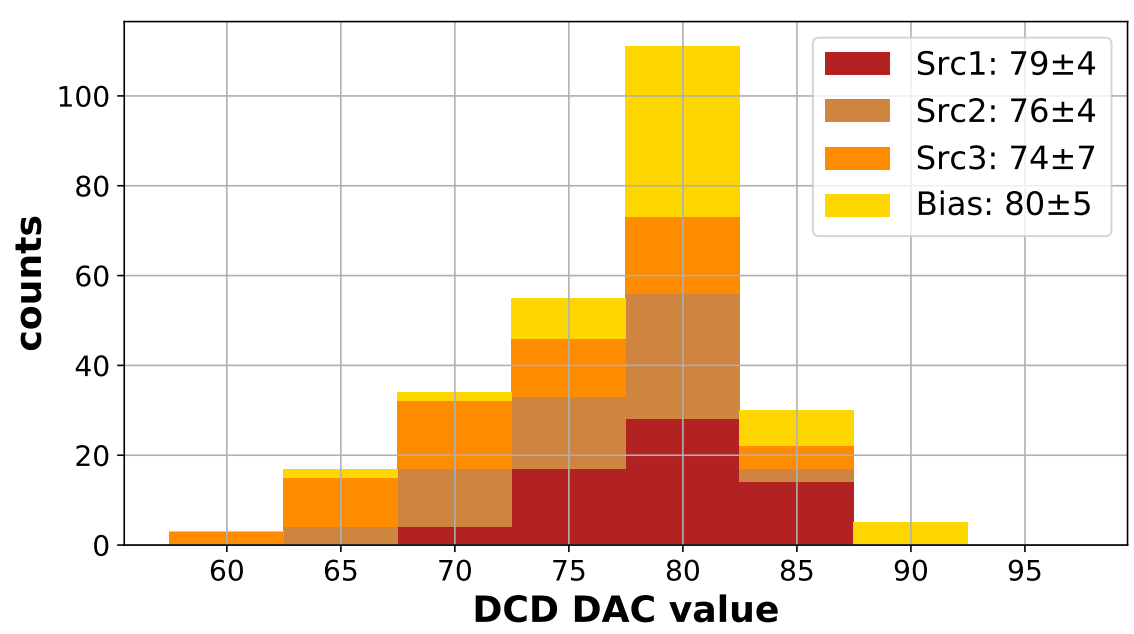

Figure 7.22.: Distribution of the optimised values for the four current source/sink DACs in the four DCDs of the modules tested at the Göttingen test stand. The individual histograms are stacked on top of each other. The legend shows the average and standard deviation of the respective distribution.

ules tested at the Göttingen test stand. Except for module W04_OB1 (as discussed above), a stable and reliable digital communication could be established. Details of the off-module high speed data link tuning are not further discussed since these are highly dependent on the actual setup (especially cable lengths) and do not provide any insight here. The optimal DCD operation parameters per chip and the optimal supply voltages per module are listed in Table 7.4. The distribution of the operation parameters and the supply voltages are shown in Figure 7.22 and Figure 7.23, respectively. A general trend in the operation parameters can be identified. The current sources/sinks Src1 and Bias tend to a value of about 80 . Src2 tends to a value of about 3 below Src1. Src3 tends to values of about 5 below Src1 and exhibits a larger variation than the other parameters in general. The supply voltage $V_{\text {RefIn }}$ is well constrained in the range $700 \mathrm{mV}$ to $750 \mathrm{mV}$. The $V_{\text {AmpLow }}$ supply on the other hand features a larger variation. Overall, the optimisation procedure yields consistent results across modules from various production wafers.

The characteristics of the effective pedestal spread at a given DEPFET gate potential $V_{\text {gate-on }}$ and DCD input amplification gain are listed in Table 7.5. Most modules can be operated at a gate potential of about $2 \mathrm{~V}$ along with an input gain of $96 \mathrm{nA} / \mathrm{ADU}$ and with analogue common mode correction (ACMC) in use. A small fraction of modules requires a more positive gate potential - and thus a smaller DEPFET internal amplification $g_{q}$ - and/or a higher DCD input gain - and thus a worse resolution of the signal charges - in order to be operated with a reasonable effective pedestal spread. A reasonable spread is given for an average of pedestals values over the pixel matrix close to $100 \mathrm{ADU}$ and a FWHM below approximately $100 \mathrm{ADU}$. Most of the tested modules 


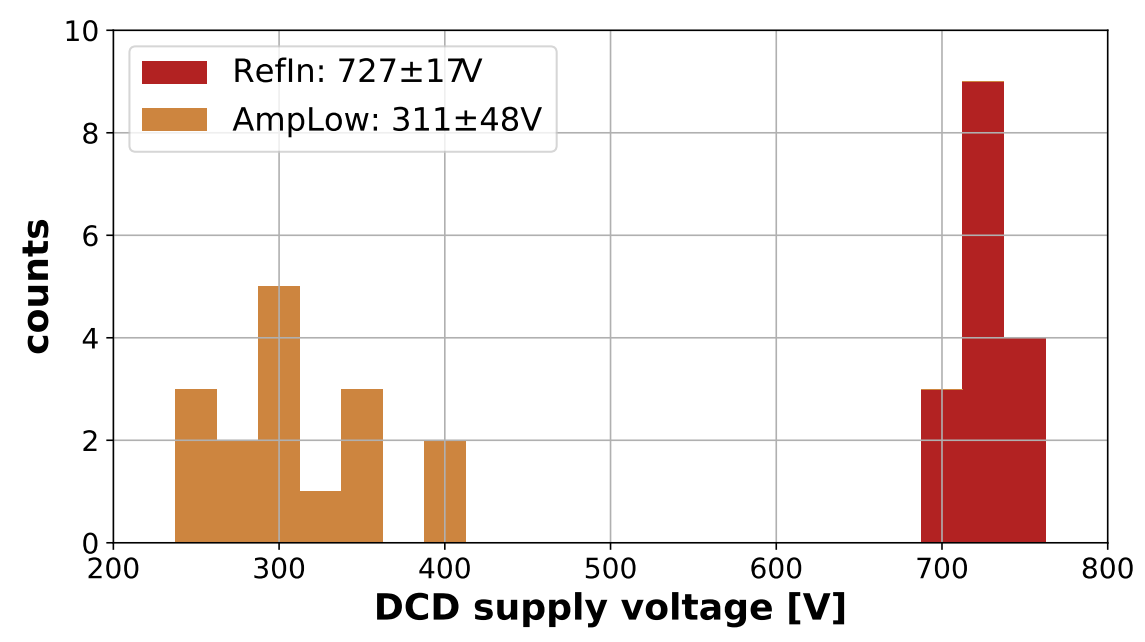

Figure 7.23.: Distribution of the optimised values for the DCD supply voltages of the modules tested at the Göttingen test stand. The legend shows the average and standard deviation of the respective distribution.

fulfil these criteria. Two modules, W06_OB1 and W04_OF1, exhibit a marginally large FWHM of the effective pedestal distribution and an increase of the DCD input gain or a decrease of the gate potential is discussable for the modules for the operation in Belle II. The average pedestal fluctuation (noise) over the pixel matrix is close to $0.8 \mathrm{ADU}$ for the majority of the modules. In three modules, W06_OB1, W03_OB1 and W04_OF1, the average noise exceeds a value of $1 \mathrm{ADU}$. The majority of pixels of the DEPFET matrix (the 95th percentile) on each module expose a noise of up to about 1.2 ADU, and up to $2.15 \mathrm{ADU}$ for the aforementioned more noisy modules. These values are sufficiently below the employed zero-suppression threshold of $5 \mathrm{ADU}$ to $7 \mathrm{ADU}$.

The response of each module to the irradiation with a $\mathrm{Sr} 90$ radioactive source is listed in Table 7.6. The optimisation of the DEPFET biasing potentials is based on the procedure proposed in the previous section. The cluster charge MPV is consistently in the range of about $20 \mathrm{ADU}$ to $30 \mathrm{ADU}$. The value of the cluster charge MPV depends on the DEPFET gate potential, the biasing scenario and possibly on variations during the wafer production (e.g. thickness of DEPFET gate oxides). The MPV values of the calibrated cluster energy distribution agree within $1 \mathrm{keV}$ with the reference value of $19.7 \mathrm{keV}$. The FWHM of the cluster energy distribution is consistently in the range of about $12 \mathrm{keV}$ to $20 \mathrm{keV}$. The calibration constants are listed in Table 7.3. The extracted SNR values are in the order of 20 to 40 . The DEPFET pixel detector modules thus yield excellent signal-to-noise ratios. The applied signal thresholds are in the order of 1000 to 2500 electrons. It should be noted, that a rather high online zero-suppression threshold is selected to obtain a clean cluster charge distribution for reliably determining the MPV of the distribution. With the low read-out noise levels observed across most of the modules, the zero-suppression threshold can presumably be reduced when the detector 


\begin{tabular}{l|cc|ccc|ccc}
\hline & \multicolumn{2}{|c}{ threshold } & \multicolumn{3}{c}{$k[\mathrm{keV} / \mathrm{ADU}]$} & \multicolumn{3}{c}{$g_{q}$ [pA/electron] } \\
module & {$[\mathrm{ADU}]$} & {$\left[e^{-}\right]$} & $P_{5}$ & avg. & $P_{95}$ & $P_{5}$ & avg. & $P_{95}$ \\
\hline W43_IB & 5 & $1000(20)$ & $0.48(1)$ & $0.60(1)$ & $0.68(1)$ & $510(60)$ & $580(60)$ & $730(60)$ \\
W03_IB & 5 & $1410(30)$ & $0.77(2)$ & $0.85(2)$ & $1.12(2)$ & $310(40)$ & $410(40)$ & $450(40)$ \\
W06_OB1 & 7 & $1450(30)$ & $0.54(1)$ & $0.65(1)$ & $0.87(1)$ & $400(60)$ & $550(60)$ & $640(60)$ \\
W12_OB2 & 7 & $2570(80)$ & $0.95(3)$ & $1.16(3)$ & $1.45(3)$ & $360(50)$ & $460(50)$ & $540(50)$ \\
W11_OF2 & 7 & $1480(30)$ & $0.61(1)$ & $0.67(1)$ & $0.81(1)$ & $430(50)$ & $520(50)$ & $570(50)$ \\
W32_OF1 & 7 & $1130(20)$ & $0.36(1)$ & $0.51(1)$ & $0.59(1)$ & $590(70)$ & $690(70)$ & $960(70)$ \\
W46_OB1 & 7 & $2020(50)$ & $0.79(2)$ & $0.91(2)$ & $1.33(2)$ & $390(60)$ & $590(60)$ & $660(60)$ \\
W41_OF1 & 7 & $2060(50)$ & $0.72(2)$ & $0.93(2)$ & $1.25(2)$ & $410(60)$ & $570(60)$ & $720(60)$ \\
W09_OB2 & 7 & $2550(80)$ & $1.00(3)$ & $1.15(3)$ & $1.36(3)$ & $380(50)$ & $460(50)$ & $520(50)$ \\
W45_OF1 & 7 & $1380(20)$ & $0.56(1)$ & $0.62(1)$ & $0.74(1)$ & $470(60)$ & $560(60)$ & $620(60)$ \\
W05_OB1 & 7 & $1940(40)$ & $0.77(2)$ & $0.87(2)$ & $1.27(2)$ & $270(40)$ & $410(40)$ & $450(40)$ \\
W03_OB1 & 7 & $1640(30)$ & $0.63(1)$ & $0.74(1)$ & $0.92(1)$ & $380(50)$ & $470(50)$ & $550(50)$ \\
W44_OB2 & 7 & $1380(20)$ & $0.57(1)$ & $0.62(1)$ & $0.73(1)$ & $470(60)$ & $560(60)$ & $610(60)$ \\
W43_OF1 & 7 & $1230(20)$ & $0.50(1)$ & $0.55(1)$ & $0.68(1)$ & $510(60)$ & $630(60)$ & $690(60)$ \\
W05_OF1 & 7 & $1810(40)$ & $0.75(2)$ & $0.81(2)$ & $0.97(2)$ & $360(40)$ & $430(40)$ & $460(40)$ \\
\hline
\end{tabular}

Table 7.3.: Calibration constants $k$, average thresholds and extracted DEPFET internal amplification $g_{q}$ factors for the modules tested at the Göttingen test stand.

modules are operated in Belle II. The extracted DEPFET amplification factors are in accordance with the aforementioned design value of $400 \mathrm{pA} /$ electron to $600 \mathrm{pA} /$ electron. Figure 7.24 displays the distribution of the extracted in-pixel amplification $g_{q}$ per fourrow per module. The fraction of non-functional pixels, $f_{\dagger}$, is well below $1 \%$ for the grade A tested modules and well below the $5 \%$ threshold for grade B tested modules.

Overall, the developed series testing procedure is a thorough test of the functionality of each detector module. The optimisation strategies for the various operation parameters yield consistent results. The overall performance of the modules exhibits excellent signalto-noise ratios and the design goals for the DEPFET amplification factors are achieved. The majority of the tested detector modules are well qualified for the installation in Belle II. 
7. Series Testing of Pixel Detector Modules for Belle II

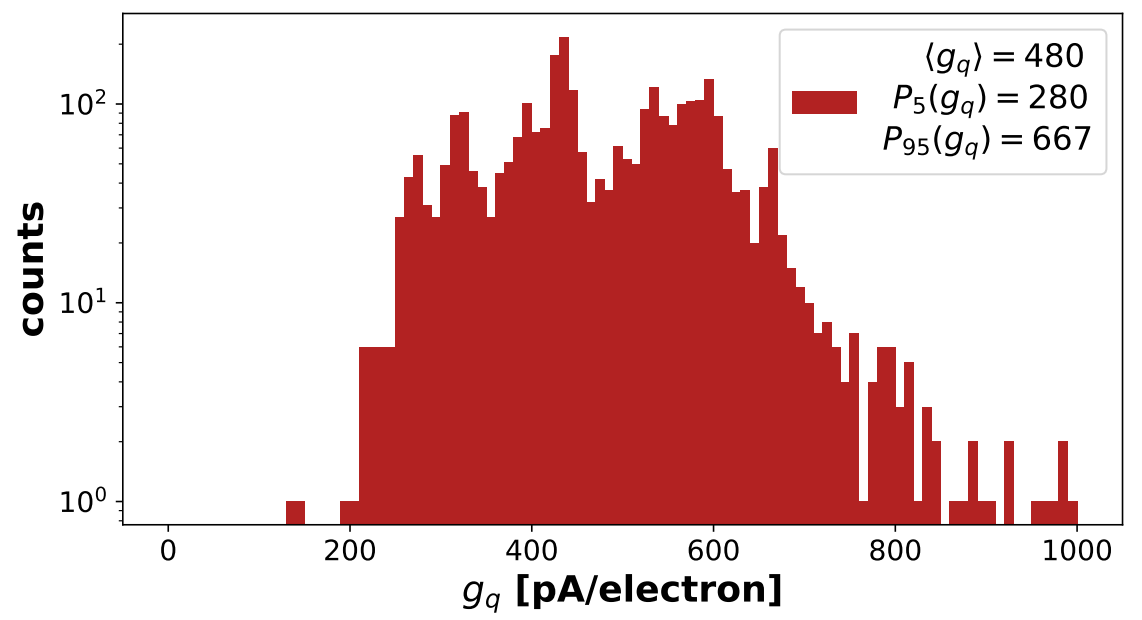

Figure 7.24.: Distribution of the in-pixel amplification $g_{q}$ measured per four-row of the modules tested at the Göttingen test stand. The legend indicates the average value and the 5th and 95th percentiles over the full distribution. 


\begin{tabular}{|c|c|c|c|c|c|c|c|c|c|c|c|c|c|c|c|c|c|c|}
\hline \multirow[b]{2}{*}{ module } & \multicolumn{4}{|c|}{ DCD1 } & \multicolumn{4}{|c|}{ DCD2 } & \multicolumn{4}{|c|}{ DCD3 } & \multicolumn{4}{|c|}{ DCD4 } & \multirow[b]{2}{*}{$V_{\text {RefIn }}$} & \multirow[b]{2}{*}{$V_{\text {AmpLow }}$} \\
\hline & Src1 & Src2 & Src3 & Bias & Src1 & $\mathrm{Src} 2$ & Src3 & Bias & Src1 & $\operatorname{Src} 2$ & Src3 & Bias & Src1 & Src2 & Src3 & Bias & & \\
\hline W43_IB & 75 & 72 & 65 & 80 & 75 & 72 & 65 & 80 & 75 & 72 & 65 & 80 & 75 & 72 & 65 & 80 & 750 & 250 \\
\hline W03_IB & 75 & 72 & 60 & 75 & 75 & 72 & 60 & 80 & 75 & 72 & 70 & 75 & 75 & 72 & 70 & 65 & 725 & 400 \\
\hline W06_OB1 & 75 & 76 & 65 & 90 & 75 & 72 & 65 & 90 & 75 & 78 & 65 & 90 & 75 & 72 & 65 & 90 & 725 & 300 \\
\hline W12_OB2 & 80 & 76 & 75 & 70 & 80 & 76 & 70 & 80 & 80 & 80 & 80 & 80 & 80 & 78 & 75 & 70 & 725 & 300 \\
\hline W11_OF2 & 80 & 78 & 70 & 80 & 75 & 72 & 70 & 80 & 80 & 80 & 80 & 80 & 80 & 78 & 65 & 80 & 700 & 250 \\
\hline W32_OF1 & 80 & 78 & 75 & 80 & 80 & 80 & 80 & 85 & 80 & 76 & 75 & 80 & 80 & 78 & 75 & 85 & 750 & 400 \\
\hline W46_OB1 & 80 & 74 & 70 & 80 & 80 & 72 & 80 & 75 & 80 & 76 & 75 & 75 & 80 & 76 & 65 & 75 & 750 & 300 \\
\hline W41_OF1 & 80 & 74 & 70 & 80 & 80 & 74 & 70 & 85 & 80 & 74 & 70 & 85 & 80 & 76 & 65 & 80 & 700 & 350 \\
\hline W09_OB2 & 80 & 80 & 80 & 80 & 80 & 80 & 80 & 80 & 80 & 80 & 75 & 75 & 80 & 76 & 75 & 80 & 700 & 300 \\
\hline W45_OF1 & 80 & 78 & 75 & 80 & 80 & 82 & 75 & 85 & 80 & 80 & 75 & 80 & 85 & 78 & 80 & 75 & 725 & 350 \\
\hline W05_OB1 & 80 & 84 & 80 & 80 & 85 & 82 & 85 & 80 & 75 & 76 & 60 & 90 & 85 & 78 & 85 & 85 & 725 & 350 \\
\hline W03_OB1 & 85 & 82 & 85 & 80 & 85 & 80 & 80 & 75 & 85 & 82 & 80 & 80 & 85 & 82 & 75 & 80 & 725 & 300 \\
\hline W44_OB2 & 85 & 80 & 80 & 80 & 85 & 84 & 85 & 85 & 85 & 82 & 80 & 85 & 80 & 78 & 70 & 80 & 725 & 275 \\
\hline W43_OF1 & 80 & 74 & 80 & 80 & 75 & 76 & 75 & 80 & 85 & 80 & 80 & 75 & 85 & 82 & 80 & 80 & 725 & 250 \\
\hline W04_OF1 & 70 & 67 & 70 & 80 & 70 & 67 & 70 & 80 & 70 & 67 & 70 & 80 & 70 & 67 & 70 & 80 & 725 & 275 \\
\hline W05_OF1 & 75 & 74 & 80 & 65 & 85 & 80 & 85 & 80 & 85 & 84 & 80 & 80 & 75 & 72 & 70 & 80 & 750 & 325 \\
\hline
\end{tabular}

Table 7.4.: Optimised DCD operation parameter per chip and supply potentials common to all four chips for all modules that underwent the analogue-digital optimisation program in the series testing at the Göttingen test stand. The potentials $V_{\text {RefIn }}$ and $V_{\text {AmpLow }}$ are given in units of $m V$. The current sources/sinks Src1, Src2, Src3 and Bias are programmable in the range 0 LSB to $127 \mathrm{LSB}$ (least significant bit). 


\begin{tabular}{l|cc|ccc|rr|rr}
\hline & \multicolumn{2}{c}{ DCD } & \multicolumn{4}{c}{ DEPFET potential [V] } & \multicolumn{3}{c}{ pedestals [ADU] } \\
module & $g_{\text {DCD }}[\mathrm{nA} / \mathrm{ADU}]$ & ACMC & $V_{\text {gate-on }}^{1}$ & $V_{\text {gate-on }}^{2}$ & $V_{\text {gate-on }}^{3}$ & avg. & FWHM & avg. & $P_{95}$ \\
\hline W43_IB & $96 \pm 10$ & $\checkmark$ & -2.00 & -2.00 & -2.00 & 126.6 & 91 & 0.86 \\
W03_IB & $96 \pm 10$ & $\checkmark$ & -2.00 & -2.05 & -2.08 & 88.1 & 65 & 0.84 & 1.15 \\
W06_OB1 & $96 \pm 10$ & - & -2.05 & -2.00 & -2.10 & 99.9 & 129 & 1.40 & 2.15 \\
W12_OB2 & $144 \pm 14$ & $\checkmark$ & -1.65 & -1.65 & -1.70 & 112.5 & 45 & 0.82 & 1.05 \\
W11_OF2 & $96 \pm 10$ & $\checkmark$ & -1.65 & -1.65 & -1.65 & 127.8 & 109 & 0.85 & 1.15 \\
W32_OF1 & $96 \pm 10$ & $\checkmark$ & -2.00 & -2.00 & -2.10 & 129.1 & 71 & 0.86 & 1.05 \\
W46_OB1 & $144 \pm 14$ & $\checkmark$ & -1.70 & -1.70 & -1.70 & 132.9 & 55 & 0.85 & 1.15 \\
W41_OF1 & $144 \pm 14$ & $\checkmark$ & -2.05 & -2.00 & -2.00 & 134.6 & 74 & 0.81 & 0.95 \\
W09_OB2 & $144 \pm 14$ & $\checkmark$ & -1.98 & -2.00 & -2.05 & 120.9 & 67 & 0.80 & 1.05 \\
W45_OF1 & $96 \pm 10$ & $\checkmark$ & -2.08 & -2.00 & -2.00 & 111.3 & 100 & 0.81 & 0.95 \\
W05_OB1 & $96 \pm 10$ & $\checkmark$ & -2.03 & -2.00 & -2.00 & 89.2 & 59 & 0.93 & 1.25 \\
W03_OB1 & $96 \pm 10$ & - & -2.00 & -2.00 & -2.00 & 68.5 & 70 & 1.13 & 1.45 \\
W44_OB2 & $96 \pm 10$ & $\checkmark$ & -1.96 & -2.00 & -1.98 & 104.8 & 94 & 0.79 & 0.95 \\
W43_OF1 & $96 \pm 10$ & $\checkmark$ & -1.99 & -2.00 & -2.06 & 111.8 & 101 & 0.80 & 0.95 \\
W04_OF1 & $96 \pm 10$ & - & -2.41 & -2.50 & -2.59 & 116.5 & 131 & 1.37 & 1.85 \\
W05_OF1 & $96 \pm 10$ & $\checkmark$ & -2.43 & -2.50 & -2.41 & 116.5 & 110 & 0.81 & 0.95 \\
\hline
\end{tabular}

Table 7.5.: Module read-out characteristics for the modules tested at the Göttingen test stand. Listed are the DCD input amplification gain, whether analogue common mode correction (ACMC) is used, the DEPFET gate potential during read-out for the three segments and the resulting pedestal spread and pedestal fluctuations (ped. noise). 


\begin{tabular}{l|ccc|ccc|ccc|c}
\hline & \multicolumn{3}{|c}{ biasing [V] } & \multicolumn{3}{c}{ Sr90 radiation } \\
module & $V_{\text {HV }}$ & $V_{\text {drift }}$ & $V_{\text {clear-off }}$ & $Q_{\text {MPV }}[\mathrm{ADU}]$ & $Q_{\mathrm{MPV}}[\mathrm{keV}]$ & FWHM [keV] & $P_{5}$ & SNR & \multicolumn{3}{c}{$\begin{array}{c}f_{\dagger} \\
{[\%]}\end{array}$} \\
\hline W43_IB & -60 & -4 & 2 & $30.4(5)$ & $19.4(2)$ & $12.0(2)$ & $17.95(5)$ & $31.68(5)$ & $93.95(5)$ & 0.21 \\
W03_IB & -60 & -6 & 3 & $24.3(5)$ & $20.2(2)$ & $14.8(2)$ & $16.35(5)$ & $26.90(5)$ & $93.35(5)$ & 0.01 \\
W06_OB1 & -60 & -6 & 3 & $29.6(5)$ & $19.0(2)$ & $14.0(2)$ & $10.55(5)$ & $19.40(5)$ & $65.95(5)$ & 2.23 \\
W12_OB2 & -60 & -5 & 2 & $18.2(5)$ & $20.6(2)$ & $14.0(2)$ & $12.25(5)$ & $20.56(5)$ & $74.75(5)$ & 1.77 \\
W11_OF2 & -60 & -6 & 3 & $28.9(5)$ & $19.1(2)$ & $12.0(2)$ & $18.15(5)$ & $31.76(5)$ & $93.05(5)$ & 1.80 \\
W32_OF1 & -68 & -5 & 2 & $34.6(5)$ & $19.5(2)$ & $12.8(2)$ & $22.25(5)$ & $36.81(5)$ & $100.45(5)$ & 0.75 \\
W46_OB1 & -60 & -5 & 2 & $23.4(5)$ & $20.0(2)$ & $13.6(2)$ & $15.15(5)$ & $25.59(5)$ & $82.55(5)$ & 0.13 \\
W41_OF1 & -60 & -5 & 2 & $21.0(5)$ & $19.7(2)$ & $14.0(2)$ & $14.95(5)$ & $25.16(5)$ & $80.45(5)$ & 0.17 \\
W09_OB2 & -62 & -5 & 2 & $19.1(5)$ & $21.2(2)$ & $15.6(2)$ & $13.75(5)$ & $23.43(5)$ & $79.25(5)$ & 0.59 \\
W45_OF1 & -68 & -3 & 4 & $32.1(5)$ & $19.7(2)$ & $14.0(2)$ & $18.25(5)$ & $35.95(5)$ & $103.75(5)$ & 0.33 \\
W05_OB1 & -58 & -4 & 3 & $26.5(5)$ & $19.2(2)$ & $13.2(2)$ & $17.55(5)$ & $28.91(5)$ & $95.25(5)$ & 25.23 \\
W03_OB1 & -60 & -3 & 4 & $25.6(5)$ & $19.2(2)$ & $19.2(2)$ & $11.55(5)$ & $21.21(5)$ & $71.35(5)$ & 1.39 \\
W44_OB2 & -60 & -5 & 2 & $32.1(5)$ & $19.6(2)$ & $13.6(2)$ & $22.95(5)$ & $40.43(5)$ & $112.75(5)$ & 0.02 \\
W43_OF1 & -60 & -5 & 2 & $34.8(5)$ & $18.7(2)$ & $12.0(2)$ & $23.35(5)$ & $39.09(5)$ & $114.75(5)$ & 0.02 \\
W05_OF1 & -70 & -5 & 5 & $24.8(5)$ & $20.1(2)$ & $15.6(2)$ & $14.35(5)$ & $29.45(5)$ & $88.75(5)$ & 0.66 \\
\hline
\end{tabular}

Table 7.6.: Response of the optimised and calibrated pixel detector modules to a Sr90 radioactive source. The choice of the three DEPFET potentials dominating the charge collection properties are given in columns two to four. The measured cluster charge MPV and FWHM is given in units of ADU and keV. The signal-to-noise ratio (SNR) distribution is outlined by giving the MPV and the 5th to 95th percentile range. The last column gives the fraction of non-functional pixels $f_{\dagger}$. 



\section{CHAPTER 8}

\section{Measuring Pixel Detector Modules in Beam Tests}

The task of a pixel detector is to recognise charged particles traversing its sensitive volume and provide precise information on the spatial coordinates of the particle passage. The detector must be precise and efficient. The precision or resolution is the uncertainty with which the spatial coordinates of a particle transition are determined. The efficiency should be close to $100 \%$, which means that (nearly) every traversing particle is detected. Several properties of a pixel detector design can be studied in small scale laboratory measurements as discussed in the precious chapter, for example charge collection characteristics using radioactive sources. The fundamental figures of merit efficiency and resolution, however, are best studied in beam test measurements. A well known and focussed beam of a single kind of charged particle with fixed momenta is pointed at the sensitive volume of the detector under test. Trajectories of individual particles in the beam are measured, such that the incidence coordinates and angles on the sensitive volume can be estimated. These particle incidences are compared to the respective detector responses, efficiency and resolution of the detector can thus be determined. For measuring the reference particle trajectories, devices usually referred to as beam telescopes are used. They consists of several planes of well characterised high resolution pixel detectors yielding precise space points of the beam particle trajectories. Particle tracks are reconstructed from the space points measured on each plane of the beam telescope (track reconstruction) and the incidence parameters on the detector under test are extrapolated.

Four pixel detector modules of the final design, as installed in the Belle II detector at the start of physics runs in 2019 [54], were measured in two beam test campaigns in November 2018 and April 2019 at the test beam facility at the DESY II electron-positron synchrotron at the DESY national research centre in Hamburg, Germany [65]. These measurements pose a significant contribution to the understanding of the performance of final production modules. These are measured here for the first time in beam tests. In 


\section{Measuring Pixel Detector Modules in Beam Tests}

the Belle II PXD, charged particles will mostly impinge on the DEPFET sensors under a non-perpendicular incidence angle, especially within the forward and backward regions. The effects of inclined particle incidence on the spatial resolution are investigated by independently rotating a pixel detector module around its two main axes in the beam. Moreover, measuring properties like efficiency with a high spatial resolution, smaller than the pixel pitches, and over the full sensor area is easily possible in a high resolution beam telescope, while the extraction from within the Belle II experiment is very challenging. One module was measured before and after X-ray irradiation, where it received a Belle II PXD lifetime ionising dose of about $266 \mathrm{kGy}$ on the DEPFET gate oxides.

In this chapter, the experimental setups are described, data processing and track reconstruction are defined and results on efficiency and resolution are presented and discussed. These beam test measurements and parts of the obtained results have been published in [66].

\subsection{Beam test measurements at the DESY test beam facility}

The beam tests were conducted at the DESY test beam facility [65] which provides a mono-energetic electron beam of $1 \mathrm{GeV}$ to $6 \mathrm{GeV}$. The facility provides an EUDETtype beam telescope for measuring reference electron tracks [67]. Infrastructure for conveniently installing and operating a detector under test and integrating its read-out into the telescope's data acquisition environment is available on-site. The DESY test beam facility is a well established instrument for measuring the performance of pixel detectors and other devices. The experimental setup and selected details on the beam telescope operation and data acquisition are presented in this section.

\subsubsection{Electron beam}

The DESY test beam facility uses the electron-positron synchrotron DESY II as a source for generating a mono-energetic beam of electrons. DESY II is a $292.8 \mathrm{~m}$ synchrotron accelerating electrons and positrons to energies of up to $6.3 \mathrm{GeV}$ [65]. A carbon fibre of $7 \mu \mathrm{m}$ thickness is placed inside the circular collider directly in the circulating beam. This generates a bremsstrahlung spectrum with energies up to $6 \mathrm{GeV}$ which is focussed on a converter target. The target converts bremsstrahlung photons to electron-positron pairs. A dipole magnet fans out the generated particles according to their momentum. A collimator after the dipole selects only particles of a certain momentum which form the test beam. The dipole polarity defines whether the test beam is formed of electrons or positrons. Only electron beams were used for the measurements presented in this thesis. Technically, the collimator is fixed and the magnet current is adjusted to select the desired test beam energy in the range of $1 \mathrm{GeV}$ to $6 \mathrm{GeV}$. Since the synchrotron energy varies over time ${ }^{1}$, the test beam intensity is not stable over time. Additional collimators for shaping the lateral dimensions of the test beam are available. The DESY

${ }^{1}$ DESY II is used as a pre-accelerator pushing electrons and positrons from $450 \mathrm{MeV}$ to momenta up to $6 \mathrm{GeV}$. 


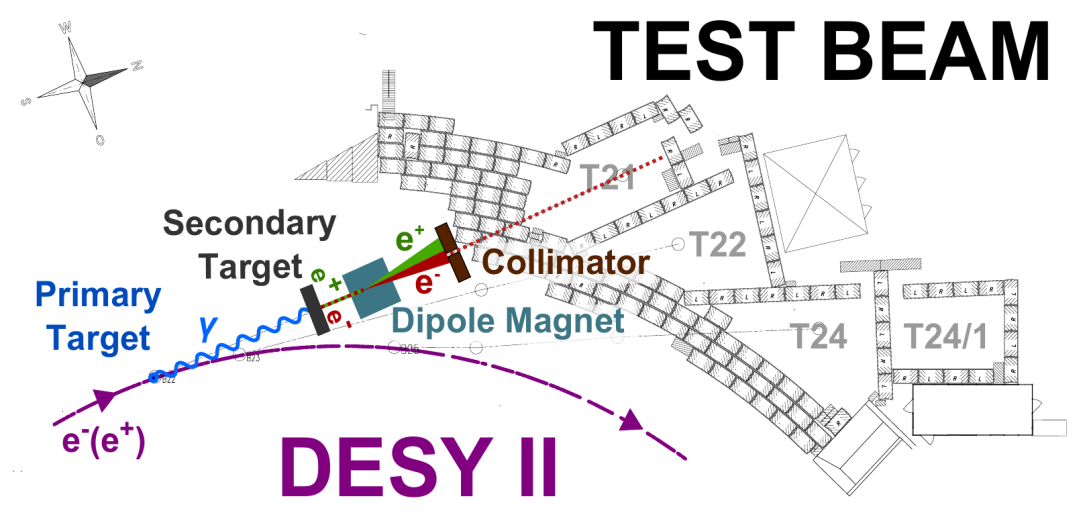

Figure 8.1.: Schematic drawing of the DESY test beam facility, sketching the beam generation for one of the three beam lines. Figure is from [65].

test beam facility provides three independent beam lines, called TB21, TB22 and TB24. The beam test campaign in 2018 was conducted at beam line TB24, while the campaign in 2019 was conducted in line TB21. Figure 8.1 sketches the beam generation and the location of the three beam lines.

The test beam is focussed with a lateral divergence of about $1 \mathrm{mrad}$. The beam particles momentum resolution depends on the particle momentum selected by the field strength of the dipole magnet. Figure 8.2 depicts the the measured resolutions, which drop below a value of $5 \%$ for momenta larger than $3 \mathrm{GeV}$. This yields a sufficiently mono-energetic particle beam and the nominal particle momentum is assumed in the reconstruction of tracks. The particle rates in the beam depend on various beam generation parameters, like the conversion target, and the selected particle momentum due to the double conversion via a bremsstrahlung spectrum. Figure 8.3 depicts the latter dependence for the three beam lines individually. The particle rate is maximised for a selected particle momentum of about $2 \mathrm{GeV}$ in each beam line. For lower and higher beam energies, the particle rate drops significantly. At the maximum, absolute particle rates are in the order of a few $\mathrm{kHz}$. Naturally, the final particle rate seen in the beam line hall also depends on the different collimator configurations. It is also clear, that the track rate, that is the rate of reconstructed tracks in the beam telescope, can be much lower than the actual particle rate and is very much dependent on the sensitivity of the trigger system, efficiencies of the telescope detectors, and so on.

\subsubsection{The EUDET beam telescope}

Measuring the trajectories of beam particles for forming reference tracks requires a beam telescope. Within the EUDET project ${ }^{2}$, a high resolution beam telescope based on precise pixel detectors has been developed [68]. These EUDET-type beam telescopes consist of six MIMOSA26 sensors, which are Monolithic Active Pixel Sensors (MAPS)

\footnotetext{
${ }^{2}$ The EUDET project concluded in 2010 , it is proceeded by the AIDA project.
} 


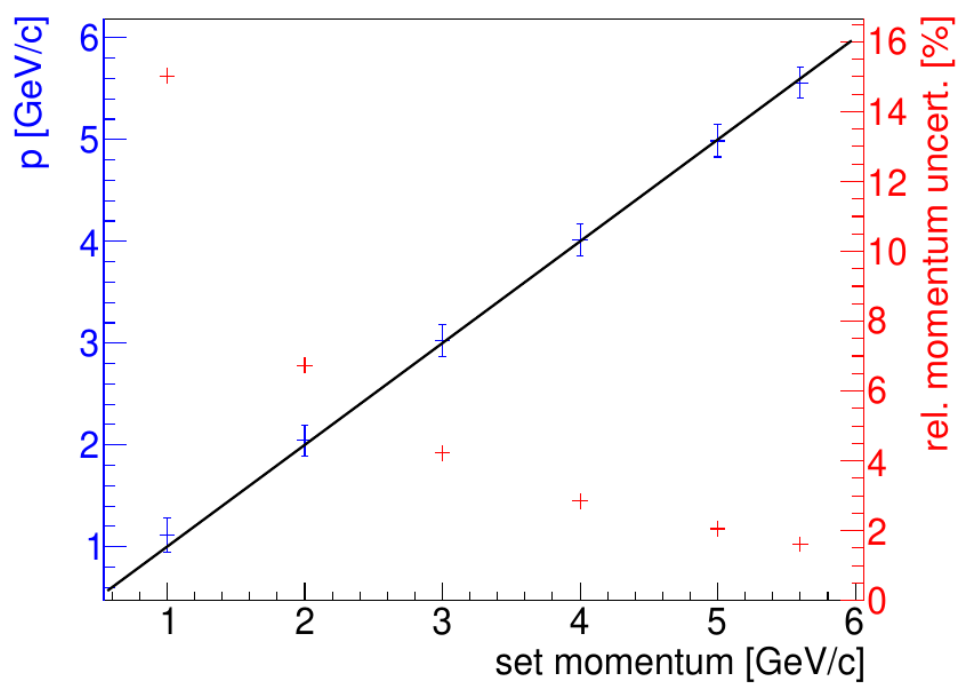

Figure 8.2.: Actual beam particle momentum (blue) and momentum resolution (red) as a function of the selected particle momentum $p$. Figure is from [65].

with $1152 \times 576$ pixels with $18.4 \mu \mathrm{m} \times 18.4 \mu \mathrm{m}$ pitch per pixel [69] (active sensor area of $10.6 \mathrm{~mm} \times 21.2 \mathrm{~mm}$ ), mechanical support structures for precise positioning of the detector planes, a Trigger Logic Unit (TLU) for triggering data read-out and a dedicated data acquisition (DAQ) software (EUDAQ) [70,71]. The infrastructure allows for straightforward integration of a device under test (DUT), a Belle II pixel detector module in this case, regarding mechanical mounting and read-out.

The small $18.4 \mu \mathrm{m}$ pixel pitch of the MIMOSA26 detectors facilitates a precise reconstruction of beam particle tracks. The planes are read out in a rolling shutter mode, where the 1152 columns are read out in parallel, while the 576 rows are selected consecutively. The read-out of all rows takes $115.2 \mu \mathrm{s}$ [69]. Zero-suppressed hit data, which are the column- and row-coordinates of pixels with a digitised signal above an adjustable threshold, is send out to the DAQ system upon receipt of a trigger signal. The MIMOSA26 detectors are mounted in aluminium housings with cut-outs below and above the sensor area, covered with $25 \mu \mathrm{m}$ lightproof Kapton foils. Together with the only $50 \mathrm{\mu m}$ thin silicon of the sensors, the total material budget per detector plane is very low, minimising the degradation of the track resolution by multiple scattering. The detectors are cooled by a water chiller, keeping the sensors at a temperature of about $18^{\circ} \mathrm{C}$.

In addition to the six MIMOSA26 planes of the EUDET-type beam telescope, a fast hybrid-pixel detector is employed. This $336 \times 80$ pixels $(50 \mu \mathrm{m} \times 250 \mu \mathrm{m}$ pixel pitch $)$ sensor is read out via an FE-I4 front-end read-out chip [72]. This detector plane, that will be referenced simply as FE-I4 in this thesis, provides a time resolution of $25 \mathrm{~ns}$ and is used as an additional telescope plane yielding spacial hit information, as well as a 

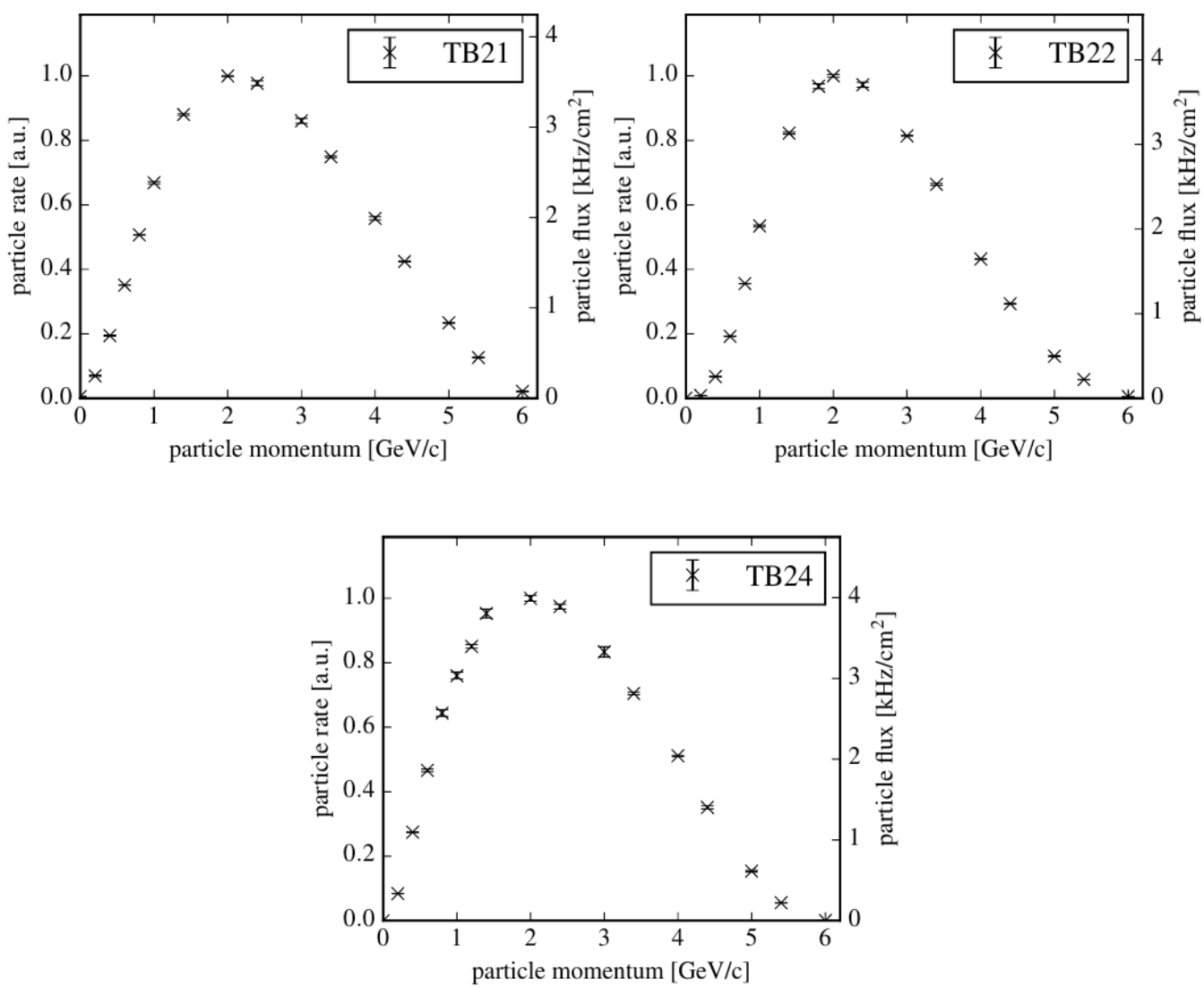

Figure 8.3.: Relative particle rate as a function of the selected particle momentum for the individual beam lines. Figures are from [65].

source for generation of trigger signals [73].

\subsubsection{Read-out and data acquisition}

The read-out of all detectors is triggered by a coincidence of the FE-I4 plane ${ }^{3}$ and a scintillator in front of the telescope. The EUDET/AIDA Trigger Logic Unit (TLU) performs the coincidence determination and trigger distribution.

The length of the MIMOSA26 and DUT read-out frames and their timing with respect to the trigger is chosen such that the hits of the triggering particle are within their respective signal integration times. The rolling shutter read-out selects one four-row segment (one row for the MIMOSA26) at a time by a read pointer which is increased in step with the rolling shutter. To receive a full matrix frame containing the possible hit data of all pixels at an instant $t_{0}$, the trigger signal has to be applied for $20 \mu \mathrm{s}$ (116 $\mathrm{ss}$ for the MIMOSA26). The full matrix frame contains hit pixels of a time span of $\pm 20 \mu \mathrm{s}$

${ }^{3}$ The FE-I4 provides an all pixel hit-or trigger, which is issued once any of its pixels shows a signal above threshold. 


\section{Measuring Pixel Detector Modules in Beam Tests}

( $\pm 116 \mu$ s for the MIMOSA26) around $t_{0}$. The lengths of the trigger signals are chosen with additional margins to account for small timing uncertainties. Any reconstructed particle track that has associated hits on the six Mimosa26 and the FE-I4 planes must have traversed the pixel detector module within its $20 \mu \mathrm{s}$ integration time window. These tracks are used for determining the hit efficiency of the pixel detector module as discussed in Section 8.6.

The online data acquisition for all detectors was integrated into the EUDAQ framework [71]. EUDAQ serves as a central run control and data collector for the individual detectors' DAQ systems. Read-out frames of each detector are collated in a single EUDAQ event with associated trigger number $N_{T}$. Consecutive events are collected in a run file. Data taking was structured in runs of $5 \times 10^{5}$ events for our beam tests. It was observed that in about every second run, a mismatch of the trigger number among the individual detectors' hit data frames within an event was present. This was corrected for by reassembling the individual hit data frames with matching trigger numbers in the offline data processing. This method of trigger number correction was validated by confirming the consistency of observables (foremost the hit efficiency, cf. Sec. 8.6) among consecutive repaired and mismatch-free runs.

\subsubsection{Telescope geometry}

The geometry and coordinate system of the beam test setup is shown in Figure 8.4.

There are two telescope arms with three MIMOSA26 sensor planes each. The FE-I4 plane is placed in the downstream arm after the last MIMOSA26-plane in direction of the beam. The distance between the telescope planes $\Delta z_{\text {tel }}$ was $2.1 \mathrm{~cm}$ when measuring perpendicular particle incidence on the DUT, and $5 \mathrm{~cm}$ when measuring inclined particle incidence. For measuring inclined incidence of the beam onto the DUT, the DUT is rotated relative to the beam telescope around the two axes perpendicular to the beam direction. A rotation around the $y$-axis is measured by the angle $\theta$, while rotations around the $x$-axis are measured by the angle $\phi$. Both rotation angles are defined as $0^{\circ}$ for perpendicular incidence. The distance between the two arms $\Delta z_{\text {DUT }}$, where the DUT is placed, was $5.8 \mathrm{~cm}$ for perpendicular incidence and up to $30 \mathrm{~cm}$ for inclined incidence, due to the dimensions of the DUT housing. Figure 8.5 shows pictures of the 2018 beam test geometry for perpendicular and inclined incidence measurements.

For perpendicular incidence, a beam energy of $3 \mathrm{GeV}$ was used, while it was increased to $5 \mathrm{GeV}$ when measuring inclined particle incidence with larger distances between the individual detector planes. Table 8.1 lists the nominal values for the distances, beam energy and DUT rotation angles as measured by rulers and the gauges of motor and rotation stages.

\subsection{Devices under test and operation}

In total, four production pixel detector modules were tested during the two beam test campaigns. All four pixel pitches that are used in the Belle II pixel detector design 


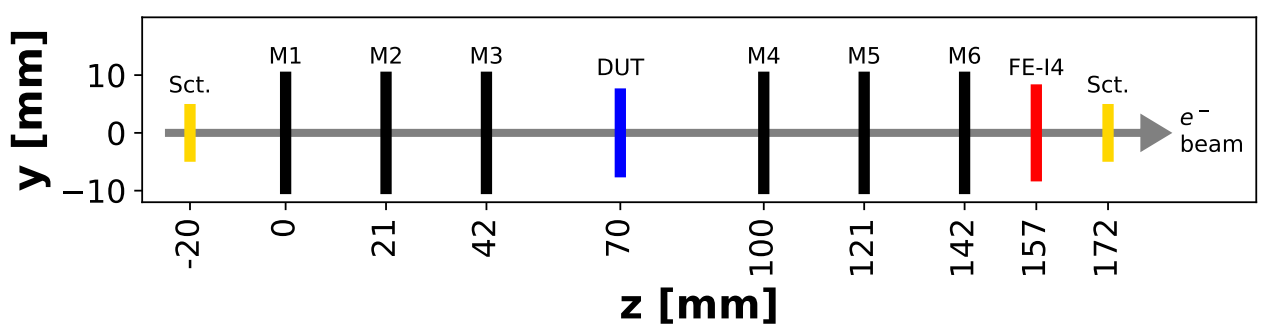

(a) perpendicular incidence

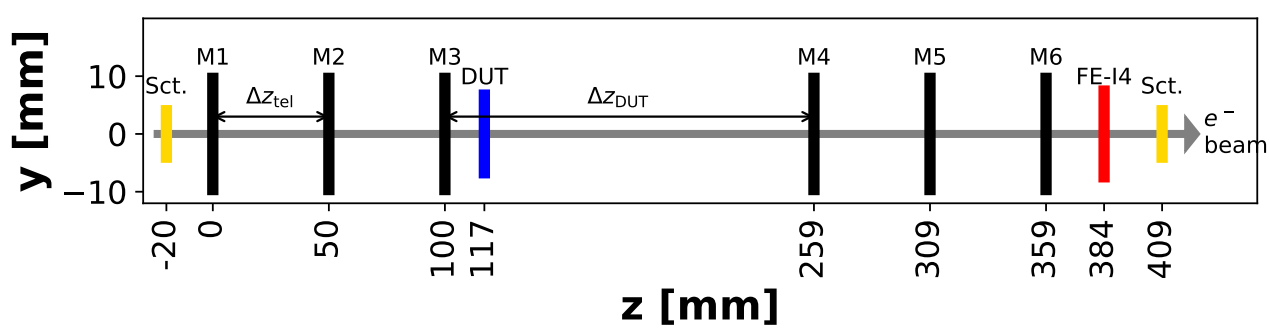

(b) inclined incidence $\left(\phi=0^{\circ}, \theta=40^{\circ}\right)$

Figure 8.4.: Sketch of the geometry of the active sensor volumes of the individual detector planes for perpendicular (a) and inclined (b) particles incidence. The labels M1 to M6 refer to the six MIMOSA26 telescope detector planes. Two scintillators (Sct.) for generating trigger signals are placed in front of the first and behind the last telescope plane. 
8. Measuring Pixel Detector Modules in Beam Tests

\begin{tabular}{ccccc}
\hline$\theta\left[^{\circ}\right]$ & $\phi\left[^{\circ}\right]$ & $E_{\text {beam }}[\mathrm{GeV}]$ & $\Delta z_{\text {tel }}[\mathrm{cm}]$ & $\Delta z_{\text {DUT }}[\mathrm{cm}]$ \\
\hline 0 & 0 & $3.0(2)$ & $2.1(1)$ & $5.8(1)$ \\
\hline 0 & 0 & $5.0(1)$ & $2.1(1)$ & $7.6(1)$ \\
0 & 20 & $5.0(1)$ & $5.0(1)$ & $7.8(1)$ \\
0 & 30 & $5.0(1)$ & $5.0(1)$ & $11.4(1)$ \\
0 & 40 & $5.0(1)$ & $5.0(1)$ & $15.9(1)$ \\
0 & 50 & $5.0(1)$ & $5.0(1)$ & $22.1(1)$ \\
0 & 60 & $5.0(1)$ & $2.1(1)$ & $30.2(1)$ \\
\hline 10 & 0 & $5.0(1)$ & $2.1(1)$ & $7.6(1)$ \\
10 & 20 & $5.0(1)$ & $5.0(1)$ & $8.6(1)$ \\
10 & 30 & $5.0(1)$ & $5.0(1)$ & $11.4(1)$ \\
10 & 40 & $5.0(1)$ & $5.0(1)$ & $15.8(1)$ \\
10 & 60 & $5.0(1)$ & $5.0(1)$ & $29.7(1)$ \\
\hline 20 & 0 & $5.0(1)$ & $2.1(1)$ & $8.6(1)$ \\
20 & 20 & $5.0(1)$ & $5.0(1)$ & $10.2(1)$ \\
20 & 30 & $5.0(1)$ & $5.0(1)$ & $13.0(1)$ \\
20 & 40 & $5.0(1)$ & $5.0(1)$ & $16.6(1)$ \\
20 & 60 & $5.0(1)$ & $5.0(1)$ & $28.4(1)$ \\
\hline 30 & 0 & $5.0(1)$ & $2.1(1)$ & $9.8(1)$ \\
30 & 20 & $5.0(1)$ & $5.0(1)$ & $11.6(1)$ \\
30 & 30 & $5.0(1)$ & $5.0(1)$ & $14.5(1)$ \\
30 & 40 & $5.0(1)$ & $5.0(1)$ & $18.1(1)$ \\
30 & 60 & $5.0(1)$ & $5.0(1)$ & $30.1(1)$ \\
\hline 40 & 0 & $5.0(1)$ & $2.1(1)$ & $10.7(1)$ \\
40 & 20 & $5.0(1)$ & $5.0(1)$ & $12.9(1)$ \\
40 & 30 & $5.0(1)$ & $5.0(1)$ & $15.6(1)$ \\
40 & 40 & $5.0(1)$ & $5.0(1)$ & $19.4(1)$ \\
40 & 50 & $5.0(1)$ & $5.0(1)$ & $24.2(1)$ \\
40 & 60 & $5.0(1)$ & $5.0(1)$ & $31.6(1)$ \\
\hline & & & & \\
\hline
\end{tabular}

Table 8.1.: Nominal DUT rotation angles, selected beam energy and distances of the MIMOSA26 detector planes and the DUT gap. 


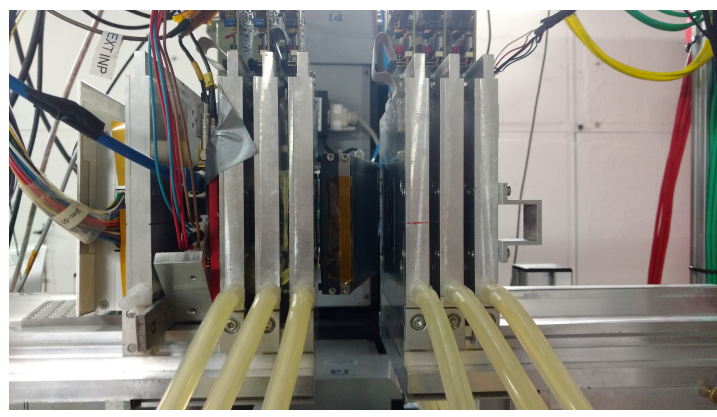

(a) perpendicular incidence

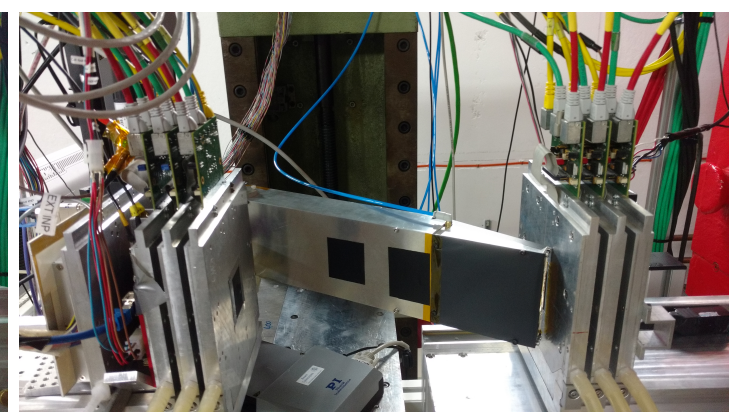

(b) inclined incidence

Figure 8.5.: Picture of the 2018 beam test geometries. The electron beam traverses the telescope from right to left.

\begin{tabular}{lcccc}
\hline name & $V_{\mathrm{HV}}[\mathrm{V}]$ & $V_{\text {drift }}[\mathrm{V}]$ & $V_{\text {clear-off }}[\mathrm{V}]$ & $V_{\text {gate-on }}[\mathrm{V}]$ \\
\hline W40_IF & -60 & -5 & 3 & 1.6 \\
W11_OF2 & -58 & -6 & 3 & -2.0 \\
W05_OB1 & -60 & -6 & 3 & -2.0 \\
W05_OB1 $\gamma$ & -60 & -6 & 3 & -10.6 \\
W37_OF1 & -62 & -6 & 3 & -4.0 \\
\hline
\end{tabular}

Table 8.2.: Biasing scenarios applied to the individual DUTs. The DEPFET gate potentials are much more negative for $\mathrm{W}_{05} \mathrm{OBB}^{\gamma}{ }^{\gamma}$ and $\mathrm{W} 37 \_\mathrm{OF} 1$ as a response to the irradiation damage of the silicon oxides.

are covered by this sample. One module, W05_OB1, was measured before and after (denoted as W05_OB1 ${ }^{\gamma}$ ) an irradiation campaign, where it was irradiated with soft Xray photons to a total ionising dose (TID) of about $266 \mathrm{kGy}$ of the DEPFET oxides [48]. The total ionising dose received by the oxides caused a shift of the FET threshold. This shift is compensated for by applying more negative FET gate potentials. The employed potentials for the measured DUTs are listed in Table 8.2. From the series testing, module W05_OB1 is known to exhibit a problematic read-out in the third ASIC pair (columns 125 to 189) (cf. Tab. 7.2). Pixels connected to this ASIC pair are thus masked already in the online read-out. Modules W40_IF (setup qualification module) and W11_OF2 were not considered for installation in the Belle II pixel detector and were thus cleared for further investigations in beam tests. Module W37_OF1 was installed and operated in the Belle II pixel detector during the beam background study phase and received an estimated TID of about $1 \mathrm{kGy}$ [62].

The pixel detector module operation in the beam tests closely resembles the laboratory setup presented in section 7.2.1. The mechanical mounting is adapted for placing the module in the beam telescope. The massive transport and cooling jigs are replaced by a thin, parylene-coated aluminium structure with a cut-out below the sensitive volume of the sensor. The module is directly screwed to the structure at the end-of-stave for 


\section{Measuring Pixel Detector Modules in Beam Tests}

mechanical fixation and for establishing a thermal contact between the module and the aluminium. In this region, the structure is more massive and, similarly to the laboratory cooling jig, has a cavity that is constantly flushed with water at $10^{\circ} \mathrm{C}$. The mounting structure in turn is placed in an aluminium housing. The housing has cut-outs below and above the sensitive volume that are covered with a light-tight foil. Condensation inside the housing and on the module is prevented by continuously flushing the inside with nitrogen. The construction is fixated to motor-stages that allow for automatic and precise (in the order of $\mu \mathrm{m}$ ) movement in the horizontal and vertical direction. Additionally, an automatic rotation-stage allows for rotations of the construction around the long side of the module (in the order of $0.1^{\circ}$ ). Rotations around the perpendicular axis are realised by manually tilting the full construction relative to the beam telescope, including the motor- and rotation-stages. The data acquisition is integrated into the EUDET infrastructure. The DHE back-end is connected the TLU and uses its signals as an external trigger source. A software plug-in interfaces the custom DAQ to EUDAQ.

During the beam test in 2018, an instability of the off-module high speed data links was observed. In a regular time interval of about $10 \mathrm{~min}$, the links dropped. This was observed consistently on all three modules investigated in the beam test in 2018 . Replacement of cables, break-out boards and the back-end hardware had no effect on the link instability. This and the regularity of the link drops suggest an external source of disturbance. In response, an automatic and fast reset of the high speed links was implemented. For mitigating effects on the analysis of the data, especially on the hit efficiency determination, active link pixels were introduced. In each read-out ASIC pair, one pixel was selected and its residual pedestal value in the DHP memory was set to zero. This results in the respective pixels to always yield a signal above the zero-suppression threshold. The recorded data is checked for the presence of these pixel hits and events are rejected in case one or more are missing.

Since the geometrical overlap of the sensitive areas of the telescope detectors (MIMOSA26 and FE-I4) and of the scintillators is in the order of $1 \mathrm{~cm} \times 2 \mathrm{~cm}$ and thus smaller than the sensor area of the DUTs, the DUTs are illuminated in several illumination windows to collect data for all DEPFET cells of the pixel matrix.

\subsection{Data processing and track reconstruction}

The processing of the recorded data is done offline after the beam test measurements. It makes extensive use of the Test Beam Software Framework [74] for reconstructing particle tracks measured by the beam telescope. Details on the track reconstruction using TBSW can be found in $[41,75]$. The data is grouped in experiments, where data of one experiment was measured for identical

- DUT configuration (threshold, biasing, etc.),

- beam energy, and

- telescope geometry (detector plane distances, DUT rotation). 
For each experiment, the data is sub-divided into runs, where one run comprises about $5 \times 10^{5}$ triggered events. Experiments are processed independently in two steps. In a first calibration step, pixel masks and the telescope alignment are determined using a subset of about $2.5 \times 10^{7}$ events (calibration sample) of the experiment. Subsequently, the track reconstruction is applied to all runs associated to the respective experiment in the reconstruction step. Figure 8.6 depicts the general process flow of both steps. The calibration process reads in a subset of events from the stored files to the given experiment. For each detector (MIMOSA26 planes, FE-I4 and DUT), a pixel mask is computed based on pixel occupancies for identifying non-functional pixels. The pixel masks are stored persistently in a pixel mask database. Masked pixels are not considered for the further analysis steps. Hit data of all non-masked pixels per detector are clustered, since hits that are neighbouring are likely caused by a single traversing particle. The masking and clustering procedure is explained in Section 8.3.1. For each cluster, the respective particle's impinging coordinate is estimated. A position finding algorithm (PFA) computes the impinging coordinates and a corresponding covariance matrix from the cluster information. The PFAs employed are discussed in Section 8.3.2. The estimated impinging coordinates of all detectors are correlated with respect to each other, yielding a coarse correction on the $x$ - and $y$-coordinates of the positions of the individual detector planes. The first telescope plane upstream is considered fixed and defines the reference frame. Calibrating corrections on the positions and rotations of the individual detector planes is called alignment and is discussed in Section 8.3.4. The position corrections are applied by shifting and tilting of the local coordinate systems of each plane. The corrected impinging coordinates at each plane are used to fit a track model. The fitted track model is the reconstructed particle track. The track fitting is based on Kalman filters [76]. Details are given in Section 8.3.3. The set of reconstructed tracks is used to further refine the corrections on the positions and rotation angles of the detector planes. A Kalman filter based alignment algorithm [77] is employed. The final set of corrections are stored persistently in an alignment database.

The reconstruction process uses the pixel mask database and applies hit clustering to all events of an experiment. The PFA estimates the impinging coordinates for the clusters and the corrections from the alignment database are applied. The final track reconstruction uses hits of only telescope planes (MIMOSA26 planes + FE-I4) for fitting of the track models. The reconstructed tracks are extrapolated to the DUT plane and associated to DUT clusters (Section 8.3.5).

\subsubsection{Pixel masking and clustering}

Individual pixels or groups of pixels of a detector can be non-functional due to failures during production or electrical or mechanical damage during operation. Non-functional pixels can be dead - not sensitive to charge deposited by traversing particles - , or hot - frequently yielding hits that are not associated to actually traversing particles. These pixels can be identified by considering the hit count $N_{i}$ of pixel $i$ or the (pixel) occupancy $\Omega_{i}$ of pixel $i$ which is the ratio $N_{i} / n_{\text {events }}$ of hit counts over a number $n_{\text {events }}$ of events. Of course, $N_{i}$ and $\Omega_{i}$ depend on the beam particle rate and the trigger rate 


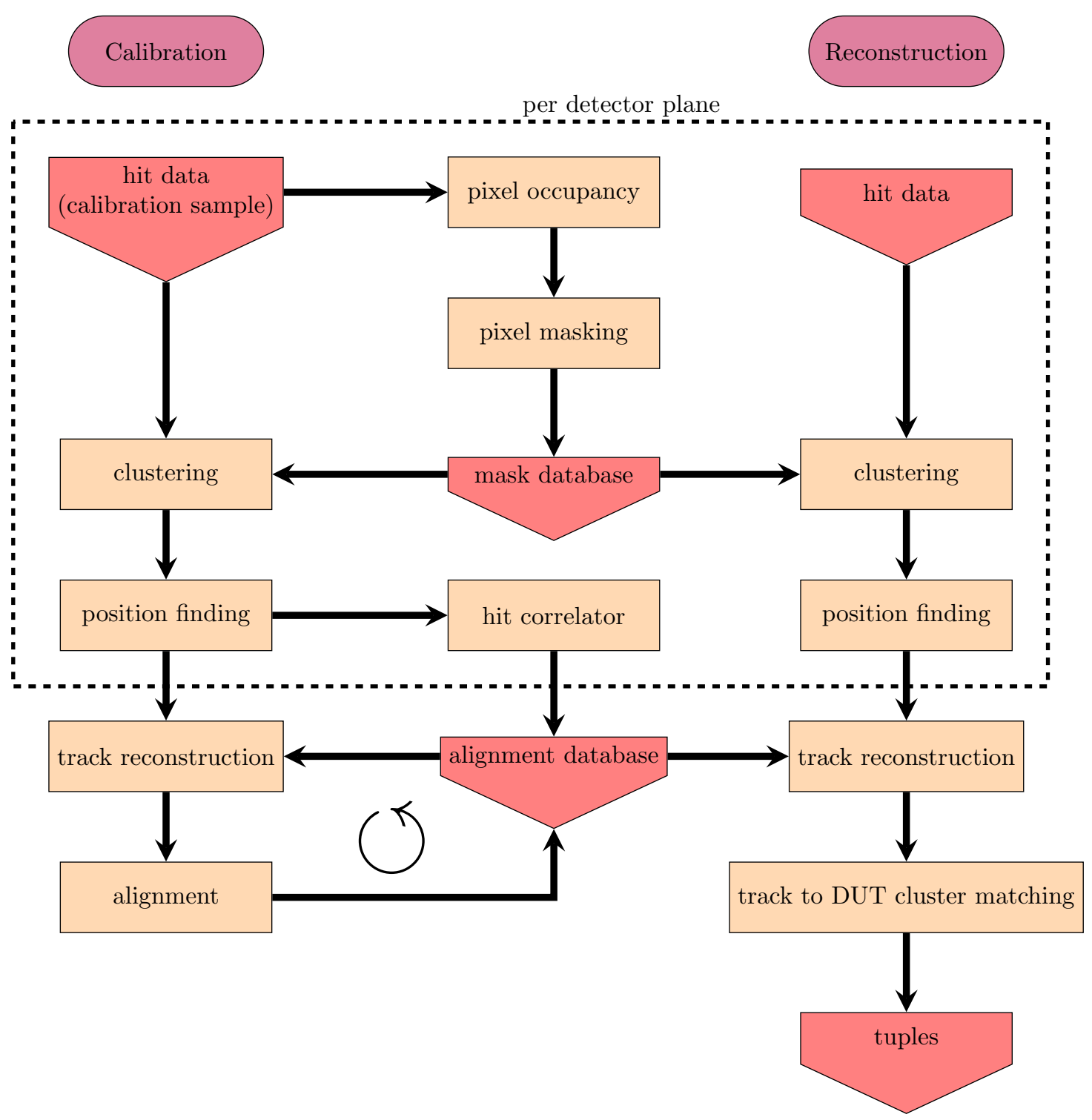

Figure 8.6.: Data calibration and track reconstruction analysis flow chart sketching the individual processing steps. The calibration is performed on a subset of the data producing pixel masks and alignment parameters. The reconstruction uses these for producing tracks for the full dataset. 


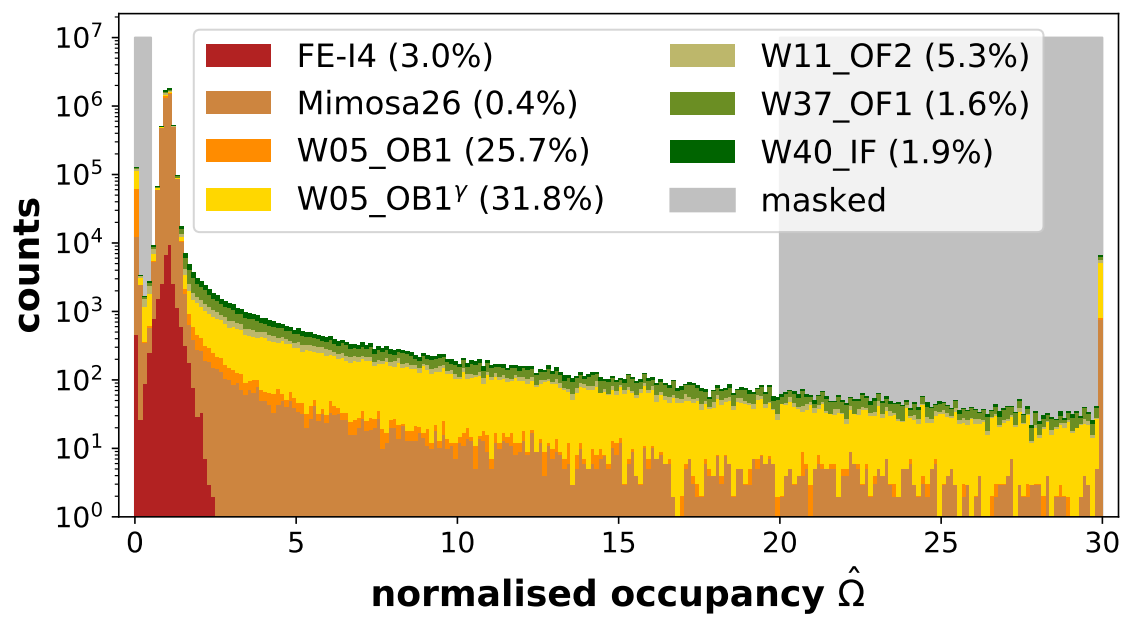

Figure 8.7.: Distribution of the normalised pixel occupancy $\hat{\Omega}$ per detector. The highest bin is an overflow bin. The six MIMOSA26 detectors are accumulated under the label "Mimosa26". The gray areas indicate the regions for identifying dead and hot pixels, respectively. The legends gives the fraction of masked pixels per device in brackets.

and can vary over the pixel matrix due to a non-uniform beam spot. For mitigating these effects, the normalised quantity $\hat{\Omega}_{i}$ is considered, which is the occupancy of pixel $i$ divided by the median occupancy of the pixels within a $5 \times 5$ grid centred at pixel $i$. Dead pixels exhibit an exceptionally low occupancy below a threshold $t_{\text {dead }}$. Hot pixels exhibit an abnormal high occupancy, relative to their neighbourhood, above a value of $t_{\text {hot }}$. Conservative thresholds of $t_{\text {dead }}=0.5$ and $t_{\text {hot }}=20$ for identifying dead and hot pixels, respectively, were chosen. Figure 8.7 shows the distribution of the normalised pixel occupancy $\hat{\Omega}$ per detector, motivating the given thresholds. It should be noted that the four artificially hot pixels for identifying link losses are contributing only by $\frac{4}{250 \cdot 768}=0.002 \%$ to the fraction of hot pixels for the DUT. For module W05_OB1 ${ }^{(\gamma)}$, before and after irradiation, the third ASIC pair and thus $25 \%$ of the pixels were masked on the online read-out level already. The average absolute occupancy is in the order of $\langle\Omega\rangle=8 \times 10^{-5}$ for the MIMOSA26 and the DUTs.

Neighbouring pixels with a signal above threshold are accumulated in pixel clusters, since pixel signals in close proximity are most likely caused by the energy deposition of a single traversing particle. Pixel signals within the same event are considered. Every pixel belonging to a cluster shares at least one edge or one corner with at least one of the remaining pixels of the same cluster. Pixels that are identified as hot or dead are always rejected from any cluster. A cluster $\mathcal{C}$ is a set of $n$ pixel signals

$$
\mathcal{C}=\left\{\left(u_{1}, v_{1}, s_{1}\right), \ldots\left(u_{n}, v_{n}, s_{n}\right)\right\}
$$

where pixel signal $i$ has coordinates $u_{i}$ and $v_{i}$ and a signal strength $s_{i}$. The hit coordinates 


\section{Measuring Pixel Detector Modules in Beam Tests}

$\left(u_{i}, v_{i}\right)$ are given by the centre of the pixel. The indexing of pixel signals is arbitrary. The cluster signal $S(\mathrm{C})$ is defined as the sum of all hit signals belonging to the cluster $S(\mathcal{C})=\sum_{i=1}^{n} s_{i}$. The cluster size $\Pi(\mathcal{C})$ is the number of pixels belonging to the cluster, $\Pi(\mathcal{C})=n$. The cluster size in $u$-direction, $\Pi_{u}$, and in $v$-direction, $\Pi_{v}$, are the maximum extent of the cluster in $u$-direction and $v$-direction, respectively. The seed pixel of the cluster is the associated pixel with the highest signal strength $s_{i}$. The pixel detector modules feature two different pixel pitches per module. The clustering is not restricted to pixels of identical pitch in a cluster.

\subsubsection{Spatial hit information from clusters}

A charged particle traversing a pixel detector deposits energy and generates charge carriers in the sensitive volume. This charge can be shared among several pixels, producing a cluster of hits on the detector. In a high-energy physics experiment, the hit cluster information is used to estimate the traversal coordinates of the impinging particle. Translating the cluster information to impinging coordinates (and their uncertainty) is the task of a position finding algorithm (PFA) [78]. The input to the PFA is a cluster $\mathcal{C}$ (Eq. 8.1), the output is an estimation of the impinging coordinates $m=(\hat{u}, \hat{v})$ with a symmetric $2 \times 2$ covariance matrix $V$. The resolution $\sigma$, that is the precision of the estimation of the coordinates, is given in the respective dimensions as the square root of the diagonals of $V$. The quantities $m$ and $V$ are used as input to the track reconstruction. The Kalman filter based track fitting requires the estimation of $m$ and $V$ to be unbiased and consistent.

Position finding by centre of gravity The centre of gravity (COG) PFA is a well studied and widely used algorithm for strip detectors, that can be applied to pixel detectors as well. It estimates the impinging coordinate as the average of the clusters hit coordinates weighted by their respective signal contribution. The impinging coordinate in $u$-direction is estimated as

$$
\hat{u}=\frac{\sum_{i=1}^{n} u_{i} s_{i}}{\sum_{i=1}^{n} s_{i}}
$$

and in $v$-direction analogously. The associated covariance matrix $V$ is constructed with the off-diagonal elements to be equal to zero. The diagonal elements of $V$ are computed as

$$
V_{11}=\left(a \frac{P_{u}}{\sqrt{12}}\right)^{2} \quad \text { and } \quad V_{22}=\left(a \frac{P_{v}}{\sqrt{12}}\right)^{2}
$$

where $P_{u}$ and $P_{v}$ are the pixel pitch in $u$ - and $v$-direction, respectively, and $a$ is a detector specific scale factor. The pixel pitches of the cluster's seed pixel are used. The scale factors for the employed detectors have been determined in previous beam tests [79] with prototype DEPFET devices and are listed in Table 8.3. The same scale factor is used for both coordinates. If not specified otherwise, presented results are based on the COG PFA. 
8.3. Data processing and track reconstruction

\begin{tabular}{lc}
\hline device & scale factor $a$ \\
\hline MIMOSA26 & 0.7 \\
FE-I4 & 1.0 \\
DEPFET & 0.8 \\
\hline
\end{tabular}

Table 8.3.: Scale factors used in the computation of the covariance matrix in the centre of gravity PFA for the individual detector types used in the beam tests.

Position finding by cluster shapes Another approach to a PFA is based on both the cluster shape and the relative distribution of the charges in the cluster pixels. This PFA is referred to as cluster shape algorithm (CSA) in this thesis. This approach was proposed and implemented by Benjamin Schwenker and first employed by Ulf Stolzenberg [41]. The cluster shape $\mathcal{S}$ is the layout of the pixels associated to a cluster $\mathcal{C}$. Technically, the cluster shape can be defined as the sequence

$$
\mathcal{S}=\left\{\left(u_{1}-O_{u}, v_{1}-O_{v}\right), \ldots,\left(u_{n}-O_{u}, v_{n}-O_{v}\right)\right\}
$$

where $\left(u_{i}, v_{i}\right)$ are the pixel coordinates (of the geometrical pixel centres), sorted in ascending order in $u_{i}$ and $v_{i}$, and $\left(O_{u}, O_{v}\right)$ is the origin of the cluster. The cluster's origin is defined as

$$
O_{u}=\min _{i=0}^{n}\left(u_{i}\right) \quad \text { and } \quad O_{v}=\min _{i=0}^{n}\left(v_{i}\right) .
$$

In case of varying pixel pitches, as present on the pixel detector modules, the cluster shape definition is extended by incorporating a pixel type $l_{i}$ for each pixel in a cluster, which is an integer value enumerating the distinct pixel designs. The task of the CSA is to compute an estimate of the impinging coordinate $m(\mathcal{S})$, relative to the cluster origin, and the $2 \times 2$ covariance matrix $V(\mathcal{S})$ for a cluster shape $\mathcal{S}$. If a pixel detector provides a measurement of the signal strength per pixel, this information is used as an additional input to the CSA. The signal ratio $\rho$ is defined as

$$
\rho=\frac{s_{\text {head }}}{s_{\text {head }}+s_{\text {tail }}},
$$

where $s_{\text {head }}$ and $s_{\text {tail }}$ are the signal strength of the head and the tail pixel of a cluster, respectively. For one-pixel clusters $(n=1)$, the signal ratio is $\rho=1 / 2$. For multipixel clusters $(n>1)$, the head and tail pixels are selected dependent on the predicted incidence angles. If the sign of the two angles is the same, the head pixel is the rightand-uppermost ${ }^{4}$ pixel of the cluster and the tail is the left-and-lowermost pixel. In case of opposite signs, the head is the left-and-uppermost pixel of the cluster and the tail is the right-and-lowermost pixel. Every distinct cluster shape $\mathcal{S}_{i}$ is then subdivided into a number of $\rho$-bins to this shape. The CSA has a tunable hyperparameter $N_{\rho}$, which

\footnotetext{
${ }^{4}$ Rightmost has precedence: Select the pixel with largest $u$-coordinate and, if multiple candidates are available, select the one with the largest $v$-coordinate of these. The left-and-lowermost pixel is selected with leftmost precedence and with lowest $u$-coordinate and $v$-coordinate, respectively.
} 


\section{Measuring Pixel Detector Modules in Beam Tests}

defines the number of non-overlapping $\rho$-bins to be considered in the subdivision of each distinct cluster shape. The CSA then provides an estimate of $m$ and $V$ per cluster shape and $\rho$-bin.

A lookup table, referred to as cluster database (clusterDB), stores the values for $m(\mathcal{S}, \rho)$ and $V(\mathcal{S}, \rho)$. Technically, the cluster shapes as keys to the lookup table are implemented as string conversions of the tuples $\left(u_{i}-O_{u}, v_{i}-O_{v}, l_{i}\right)$ that are concatenated. When employing the CSA in the event reconstruction, a cluster $\mathcal{C}$ is fed into the CSA. The cluster shape $\mathcal{S}(\mathcal{C})$ and the signal ratio $\rho(\mathcal{C})$ of the supplied cluster are computed as described above and the cluster database is searched for the combination, yielding the respective estimates if present in the database. If there is no match found, then $m(\mathcal{S}, \rho)$ and $V(\mathcal{S}, \rho)$ are computed according to the COG PFA as a fallback.

The CSA requires a data sample for calibration and creation of the cluster database content. The calibration sample is a dataset $D$ containing a number of $n_{\mathcal{C}}$ clusters together with a predicted intersection $t$ associated to each cluster

$$
D=\left\{\left(\mathcal{C}_{1}, t_{1}, T_{1}\right), \ldots\right\},
$$

where $t$ is a vector of predicted local intersection coordinates and incidence angles $\left(u^{\text {pred }}, v^{\text {pred }}, \phi^{\text {pred }}, \theta^{\text {pred }}\right)$ and $T$ is the covariance matrix of these predictions. From all clusters $\mathcal{C}_{i} \in D$, a set of $n_{\mathcal{S}}$ distinct cluster shapes $\mathcal{S}_{i}$ is extracted. If for a specific shape $\mathcal{S}_{i}$, the number of clusters, $n_{\mathcal{S}}\left(\mathcal{S}_{i}\right)$, of that shape found in the dataset is below a threshold of $N_{\mathcal{e}}$, then this specific shape is not considered for the cluster database (i.e. the COG PFA is used as fallback). The threshold $N_{\mathcal{C}}$ is another hyperparameter of the CSA and is usually set to a number $\mathcal{O}\left(N_{\mathcal{C}}\right)=100$. For all shapes with sufficient occurrence, the dataset is split into shape specific subsets $D\left(\mathcal{S}_{i}\right)$. The signal ratios $\rho$ of all clusters belonging to a subset are computed. Equal frequency binning is applied to the distributions of $\rho$ values to find a $\rho$-binning per shape $\mathcal{S}$. The binning procedure starts with a number $N_{\rho}$, the other hyperparameter of the CSA, of $\rho$-bins. However, it is required that now every $\rho$-bin comprises at least a number of $N_{\mathcal{C}}$ of clusters. If this cannot be attained with $N_{\rho}$ bins, the number of bins is reduced to meet the $N_{\mathcal{C}}$ threshold in each $\rho$-bin. Also, the number of $\rho$-bins for the one-pixel cluster shape is always set to 1. Each subset is subsequently further divided into a set $D\left(\mathcal{S}_{i}, \rho_{i}\right)$ of specific shape and $\rho$-bin. These sets are used to calibrate the estimates $m(\mathcal{S}, \rho)$ and $V(\mathcal{S}, \rho)$ of the cluster database as

$$
m\left(\mathcal{S}_{i}, \rho_{i}\right)=\left\langle\left(u^{\text {pred }}-O_{u}, v^{\text {pred }}-O_{v}\right)\right\rangle
$$

and

$$
V\left(\mathcal{S}_{i}, \rho_{i}\right)=\operatorname{Cov}\left(\left(u^{\text {pred }}-O_{u}, v^{\text {pred }}-O_{v}\right)-m\left(\mathcal{S}_{i}, \rho_{i}\right)\right)-\langle T\rangle,
$$

where the average and covariance are computed over the respective quantities in $D\left(\mathcal{S}_{i}, \rho_{i}\right)$ (indices omitted for readability).

In a beam test experiment, the predictions come from particle tracks reconstructed using information of a beam telescope. There, $t$ is the extrapolated track intersection at a detector plane and $T$ is the uncertainty of this prediction. The high resolution EUDET beam telescope facilitates precise predictions of the track intersection on a DUT. If the 
CSA is to be used for all detectors, including the telescope planes, the calibration can be performed iteratively. The procedure starts with an initial reconstruction of tracks of the data sample based on the COG PFA estimation of the impinging coordinates on each detector plane. A first lookup table of CSA estimates is computed from these. The improved CSA estimation of impinging coordinates is used to again reconstruct tracks of the data sample. In turn, the lookup table is updated and so forth. Eventually, the procedure converges and the final estimates in the lookup table are adopted. A sixfold iteration is employed for the CSA calibration in the beam tests. The six identical MIMOSA26 detector planes share a common lookup table, where the calibration of the estimates is based on data of the four inner MIMOSA26 planes only.

\subsubsection{Track finding with Kalman filters}

Tracks models are fitted to the estimated impinging coordinates (respecting their covariance matrices) obtained by the PFA by employing Kalman filters [76]. The Kalman filter is the optimal recursive estimator for the state vector for a linear dynamic system with Gaussian measurement noise [80]. In the context of track fitting, the state is a beam particles position and direction of movement at the detector planes. The state vector $\lambda_{k}$ at plane $k$ is given by

$$
\lambda_{k}=(u, v, \tan \phi, \tan \theta),
$$

where $u$ and $v$ are the intersection coordinates and $\tan \phi=\mathrm{d} u / \mathrm{d} w$ and $\tan \theta=\mathrm{d} v / \mathrm{d} w$ are the incidence angles in the directions given by the direction tangents of the linearised track model at the intersection. The linear dynamic system is the evolution of this track state from one plane to the next. In the absence of a magnetic field, as it is the case for the reported beam test measurements, the particle hypothesis is an electron on a linear trajectory with a momentum equal to the beam energy. The track model $f_{k \mid k-1}$ thus propagates the track state from plane $k-1$ to plane $k$ on a straight line. It incorporates multiple scattering effects at plane $k-1$ by including scattering angles $\varphi_{k-1}$ and $\vartheta_{k-1}$ computed by an appropriate scattering model ${ }^{5}$ as discussed in [81]. Multiple scattering in the air between planes is integrated as well as intermediate "planes of air". The Kalman filter propagates the track state from plane $k-1$ to plane $k$, yielding a track state prediction $\lambda_{k}^{\text {pred }}$ with predicted covariance matrix $C_{k}^{\text {pred }}$. The track state prediction is then updated with the measured impinging coordinates $m_{k}$ (and covariance matrix $V_{k}$ ) of detector plane $k$. If multiple hit clusters are measured on detector $k$, the one with the impinging coordinates closest to the predicted intersection coordinates is selected. The distance between the impinging coordinates and the predicted intersection coordinates is given by the residual $r_{k}$ defined as

$$
r_{k}=m_{k}-H \lambda_{k}^{\text {pred }}
$$

and the residual covariance matrix $R_{k}$ defined as

$$
R_{k}=V_{k}+H C_{k}^{\text {pred }} H^{T},
$$

\footnotetext{
${ }^{5}$ The material composition and geometrical distribution of each detector plane has to be provided.
} 


\section{Measuring Pixel Detector Modules in Beam Tests}

where the $2 \times 4$ matrix $H$ projects the track state onto local plane coordinates. The matrix $T=H C_{k}^{\text {pred }} H^{T}$ is the precision of the extrapolation, referred to as pointing resolution. Only hit clusters with a residual value below $400 \mu \mathrm{m}$ in both $u$ - and $v$-direction are considered for updating the track state. An incremental $\chi^{2}$ value is computed as

$$
\chi_{k, \text { inc }}^{2}=r_{k}^{T} R_{k}^{-1} r_{k}
$$

and measures the statistical compatibility of the measurement $m_{k}$ with the prediction $\lambda_{k}^{\text {pred }}$. A threshold of $\chi_{k \text {,inc }}^{2}<20$ is applied for rejecting outlier hits. The updated track state $\lambda_{k}$ and covariance matrix $C_{k}$ are computed with the Kalman gain matrix method [76] using the selected cluster's $m_{k}$ and $V_{k}$. If no cluster is selected, the track state is not updated and simply extrapolated to the following plane $k+1$ (but including the the multiple scattering contribution from plane $k$ ).

The procedure is iterated through the telescope planes up to the last downstream plane (the FE-I4 in this case). The track $\chi^{2}$ value is computed as the sum over the incremental $\chi_{\text {inc }}^{2}$ values,

$$
\chi^{2}=\sum_{k} \chi_{k, \text { inc }}^{2}
$$

A backward pass of the Kalman filter, from the last telescope plane to the first in beam direction, yields a second set of track states $\lambda_{k}^{\prime}$. At plane $k$, the final estimated track state is the average of $\lambda_{k}$ (forward pass) and $\lambda_{k}^{\prime}$ (backward pass) weighted by $C_{k}$ and $C_{k}^{\prime}$, respectively. The residuals $r_{k}$ and residual covariance matrices $R_{k}$ for the weighted predictions are updated according to Eq. 8.11 and Eq. 8.12. The track $\chi^{2}$ (Eq. 8.14) is updated as well and reconstructed tracks exceeding a $\chi^{2}$ value of 100 are rejected from further analysis.

\subsubsection{Telescope alignment}

The nominal positions and rotations of the detector planes are measured by scales and rulers, or more precisely by the gauge of the motor stages where applicable. However, the resolutions of these measurements are not good enough to reliably reconstruct track states within a few $\mu \mathrm{m}$ precision. An alignment procedure, effectively employing the particle beam as a gauge, is applied for computing corrections to the nominal telescope geometry. The individual plane's positions and orientations are essential for the transformation of global coordinates $r=(x, y, z)$ to sensor coordinates $q=(u, v, w)$ as $q=R\left(r-r_{0}\right)$ and vice versa. The plane's nominal position and orientation enter into the quantity $r_{0, k}$, which is the origin, and the $3 \times 3$ rotation matrix $R_{k}$ of plane $k$. The plane origin is defined as the position of the centre of the fiducial volume of the respective sensor. A detector plane is treated as an inelastic body. Corrections from the alignment procedure are applied as $r_{0} \rightarrow r_{0}+\Delta r$ for a correction vector $\Delta r=(\Delta x, \Delta y, \Delta z)$ of small magnitude and $R \rightarrow R \Delta R$ for a correction rotation matrix $\Delta R$ with elements of small magnitude [77]. In the approximation of small corrections to the plane rotation, the correction rotation matrix is decomposed as $\Delta R=\Delta R_{\alpha} \Delta R_{\beta} \Delta R_{\gamma}$ in three correction rotation matrices describing rotations around the three coordinate axes $u(\Delta \alpha), v(\Delta \beta)$ 
and $w(\Delta \gamma)$ exclusively. This sums up to six corrections $a=(\Delta x, \Delta y, \Delta z, \Delta \alpha, \Delta \beta, \Delta \gamma)$ per plane.

In a first step, the impinging coordinates of clusters estimated by the PFA on each sensor $k$ are transformed into the global coordinate system using the respective nominal plane position and orientation. For each plane, the impinging $x$ coordinates are correlated with the impinging $x$ coordinates on the first telescope plane. Any bias in the distribution of correlation coefficients is directly used as the value for the $\Delta x$ correction of the respective plane. The first detector plane thus defines the global reference frame. A value for the $\Delta y$ correction is found analogously by correlating the impinging $y$ coordinates. With this initial set of alignment corrections, a track reconstruction is performed as described in section 8.3.3, without applying thresholds to $\chi_{k \text {,inc }}^{2}$ and $\chi^{2}$ and using a residual threshold of $500 \mu \mathrm{m}$. Tracks are required to have an associated cluster on at least seven of the eight detector planes. Given a set of reconstructed tracks, a global alignment $\chi^{2}$ function can be defined as

$$
\chi_{\text {align }}^{2}=\sum_{t}^{\text {tracks planes }} \sum_{k}^{2} \chi_{k, \text { inc }}^{2}+\left(a^{k}-a_{0}^{k}\right)^{T} W_{0}^{k}\left(a^{k}-a_{0}^{k}\right)
$$

where $\chi_{k, \text { inc }}^{2}$ are the incremental track $\chi^{2}$ values of track $t$ (Eq. 8.14), $a^{k}$ is the set of six alignment corrections of plane $k, a_{0}$ is the initial reference set of alignment corrections of plane $k$ and $W_{0}$ is the inverse of the covariance matrix of $a_{0}$. The matrix $W_{0}$ is taken to be diagonal with diagonal elements to be equal to zero for alignment corrections to be fixed. The Kalman Alignment Algorithm with Annealing [82] is used for minimising $\chi_{\text {align }}^{2}$. It is a sequential method, reconstructing and processing tracks iteratively, where the current track is reconstructed based on the updated alignment corrections found by the previous track. Annealing means that the weights of the measurements are small in the beginning and are gradually increased to a value of 1 with the number of processed tracks. All alignment corrections of the first and last MIMOSA26 plane are fixed. In addition, the rotation angles $\Delta \alpha$ and $\Delta \beta$ are fixed for all planes, except for the DUT. The Kalman alignment is repeated three times with increased track quality requirements with thresholds on the residuals $(<400 \mu \mathrm{m})$, the incremental $\chi_{k, \text { inc }}^{2}(<20)$ and the track $\chi^{2}(<100)$.

\subsubsection{Track extrapolation to the DUT}

With the alignment corrections at hand, the track reconstruction is performed on the full dataset of one experiment. For this turn, clusters from the DUT plane are explicitly excluded and not considered in the Kalman filter passes. Tracks are required to have associated clusters on at least six of the remaining seven (six MIMOSA26 + FE-I4) detector planes. Clusters are considered if the residuals in $u$ - and $v$-direction are smaller than $400 \mu \mathrm{m}$ and the increment $\chi_{k, \text { inc }}^{2}$ is below a value of 20 . Tracks exceeding an overall $\chi^{2}$ of 100 are rejected. Each reconstructed track yields a prediction of the intersection coordinates with the DUT plane. These are compared to the impinging coordinates of clusters present on the DUT, where only clusters are considered with a residual below 


\begin{tabular}{lccccrc}
\hline DUT & $E_{\text {beam }}[\mathrm{GeV}]$ & $Q_{\mathrm{MPV}}[\mathrm{keV}]$ & $\langle k\rangle[\mathrm{keV} / \mathrm{ADU}]$ & $\langle$ thrsd. $\rangle\left[10^{3} e^{-}\right]$ & masked $[\%]$ & $\langle\Pi\rangle$ \\
\hline W40_IF & $3.0(2)$ & $19.9(5)$ & $1.00(8)$ & $1.11(9)$ & 1.87 & 1.36 \\
W11_OF2 & $3.0(2)$ & $19.7(5)$ & $0.68(4)$ & $0.76(4)$ & 5.35 & 1.49 \\
W05_OB1 & $3.0(2)$ & $19.8(5)$ & $0.76(7)$ & $0.84(8)$ & 25.69 & 1.44 \\
W05_OB1 $^{\gamma}$ & $2.0(2)$ & $19.5(5)$ & $0.62(4)$ & $0.69(5)$ & 31.82 & 1.39 \\
W37_OF1 & $2.0(2)$ & $18.2(5)$ & $0.49(1)$ & $0.54(1)$ & 1.64 & 1.56 \\
\hline
\end{tabular}

Table 8.4.: DUT response to the electron beam of energy $E_{\text {beam }}$ at perpendicular incidence and extracted calibration constants. The calibration constant $k$ and threshold are given as the average and standard deviation over the pixel matrix four-rows.

$200 \mu \mathrm{m}$ in both $u$ - and $v$-direction. If multiple clusters are qualified, the one with the smallest euclidean distance is selected. The result is a list of reconstructed tracks with their respective quality figures $\left(\chi^{2}, \chi_{k \text {,inc }}^{2}\right)$ and extrapolated intersection coordinates $\left(u^{\text {pred }}, v^{\text {pred }}\right)$ and intersection angles $\left(\tan \phi^{\text {pred }}, \tan \theta^{\text {pred }}\right)$ and a possibly matched DUT cluster with its cluster signal, seed signal, size and impinging coordinate as determined by the PFA. The analyses presented in the following are based on these tuples.

\subsection{Module response to electron beam}

Analogously to the Sr90 radioactive source measurements of the series testing, as discussed in section 7.2.5, the modules' response to the beam particles exposure are investigated. During the beam test in 2018, the modules W11_OF2, W40_IF and W05_OB1 were exposed to a $3 \mathrm{GeV}$ electron beam at perpendicular incidence. In the beam test in 2019 , the modules W05_OB1 ${ }^{\gamma}$ and W37_OF1 were exposed to a $2 \mathrm{GeV}$ electron beam at perpendicular incidence. A reference energy deposition spectrum is simulated using Geant4 (cf. section 7.2.5). The simulation employs a mono-energetic electron source of energy $E_{\text {beam }}$ emitting particles that impinge on a $75 \mu \mathrm{m}$ thick silicon material at perpendicular incidence. The attenuation of the beam energy due to traversal of air and three MIMOSA26 detector planes is not factored in. The simulation is performed for beam energies of $2 \mathrm{GeV}$ to $5 \mathrm{GeV}$ and the MPV of the energy deposition, $\hat{Q}_{\mathrm{MPV}}$, is determined by fitting a Landau distribution to the histogrammed energy deposition values. The uncertainty on $\hat{Q}_{\mathrm{MPV}}$ is estimated as half the bin width. Only a minor dependence of $\hat{Q}_{\mathrm{MPV}}$ on $E_{\text {beam }}$ is found as shown in Figure 8.8. For a beam energy of $E_{\text {beam }}=3 \mathrm{GeV}$, a reference value of $19.61(5) \mathrm{keV}$ is determined. The reference value $\hat{Q}_{\mathrm{MPV}}$ is used to determine calibration factors $k$ per four-row for each module. The measured energy deposition MPV, $Q_{\mathrm{MPV}}$, is extracted from the distribution of the calibrated cluster energy values via fitting of a LanGau distribution. The online zero-suppression threshold, in units of ADU, is converted to a signal threshold in units of number of electrons for each four-row individually. Table 8.4 summarises the values of $Q_{\mathrm{MPV}}$, calibration factors, thresholds, fraction of masked pixels and average cluster sizes $\langle\Pi\rangle$ measured for the individual DUTs. Figure 8.9 shows representative distributions of calibrated cluster energy 


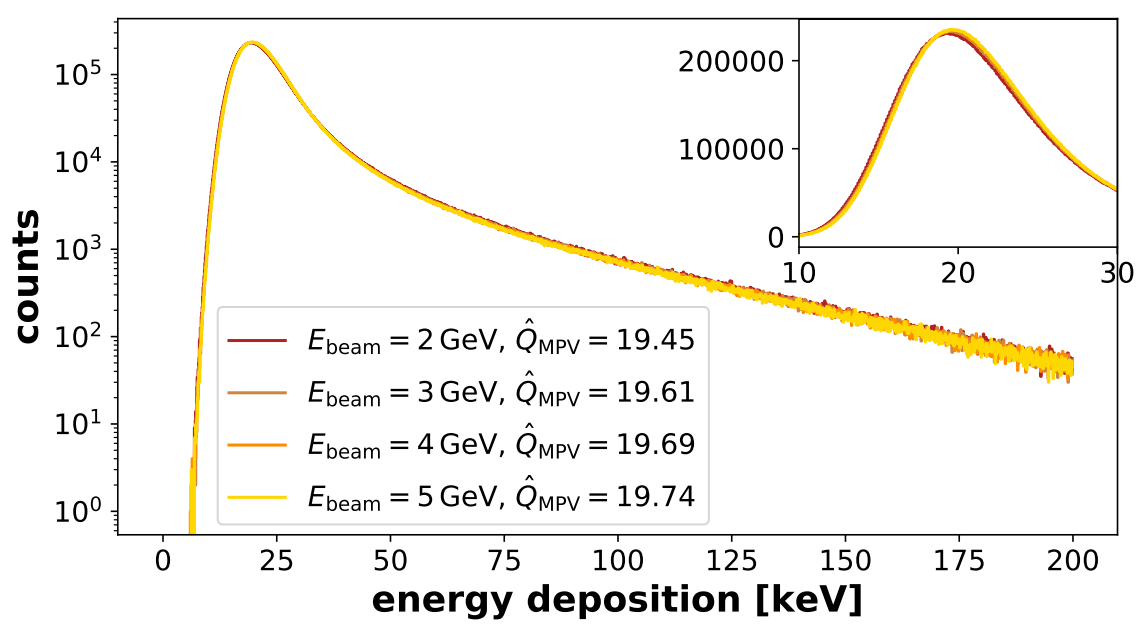

Figure 8.8.: Simulation of the energy deposition of electrons of energy $E_{\text {beam }}$ in $75 \mu \mathrm{m}$ silicon, histogrammed in bins of $0.1 \mathrm{keV}$ width in the range $0 \mathrm{keV}$ to $200 \mathrm{keV}$. The respective MPV, $\hat{Q}_{\mathrm{MPV}}$, is determined by fitting a Landau distribution in the range $10 \mathrm{keV}$ to $40 \mathrm{keV}$.

and cluster size at perpendicular incidence for module W40_IF. The LanGau fit yields $Q_{\mathrm{MPV}}=19.9(5) \mathrm{keV}$. A majority of $69.3 \%$ of one-pixel clusters is measured. Two-pixel clusters make up a fraction of $27.3 \%$. The average total cluster size is $\langle\Pi\rangle=1.36$. Clusters with a total size larger than 20 are occasionally found - in this example they make up a fraction of about $10^{-7}$. An example of a cluster of size 21 is shown with absolute pixel signals. The extrapolated track intersection coincides with the seed pixel coordinate (pixel with highest signal). Due to the phenomenology, these clusters are attributed to $\delta$-electron events, where the traversing electron knocks out a bound electron from its shell. This recoil electron strays around in the sensitive volume producing a long tail of pixel signals.

\subsubsection{Inclined particle incidence and charge sharing}

Inclined particle incidence was measured during the beam test 2018 with module W40_IF. In this scenario, the charged particle travels a longer distance inside the sensitive volume of the detector and thus deposits a larger amount of energy and generates more charges. Due to the inclination, the charge cloud has a larger extent and the charge is more likely to be spread over multiple pixels. This is called charge sharing. From basic geometrical considerations, the total path length the electrons travel in the sensitive volume depends on the incidence angles as

$$
l(\phi, \theta)=l_{0} \sqrt{1+\tan ^{2} \phi+\tan ^{2} \theta},
$$

where $l_{0}=75 \mu \mathrm{m}$ is the path length at perpendicular incidence and is equal to the thickness of the depleted silicon bulk. The most probable energy loss per distance, 


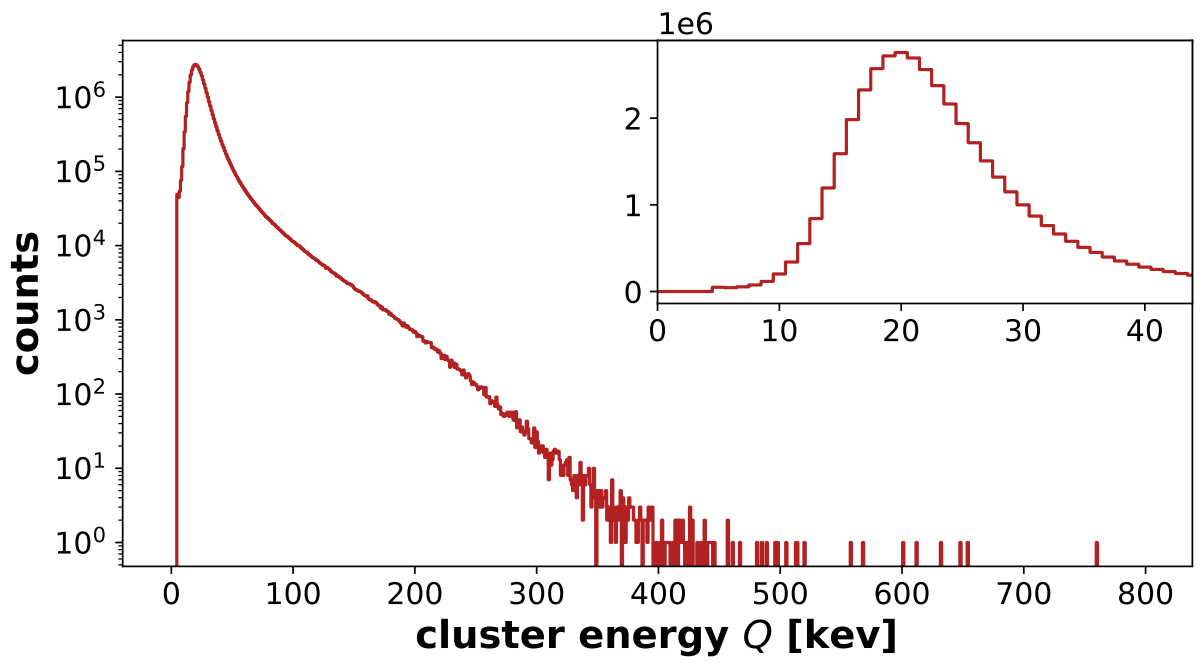

(a) energy deposition of $3 \mathrm{GeV}$ electrons

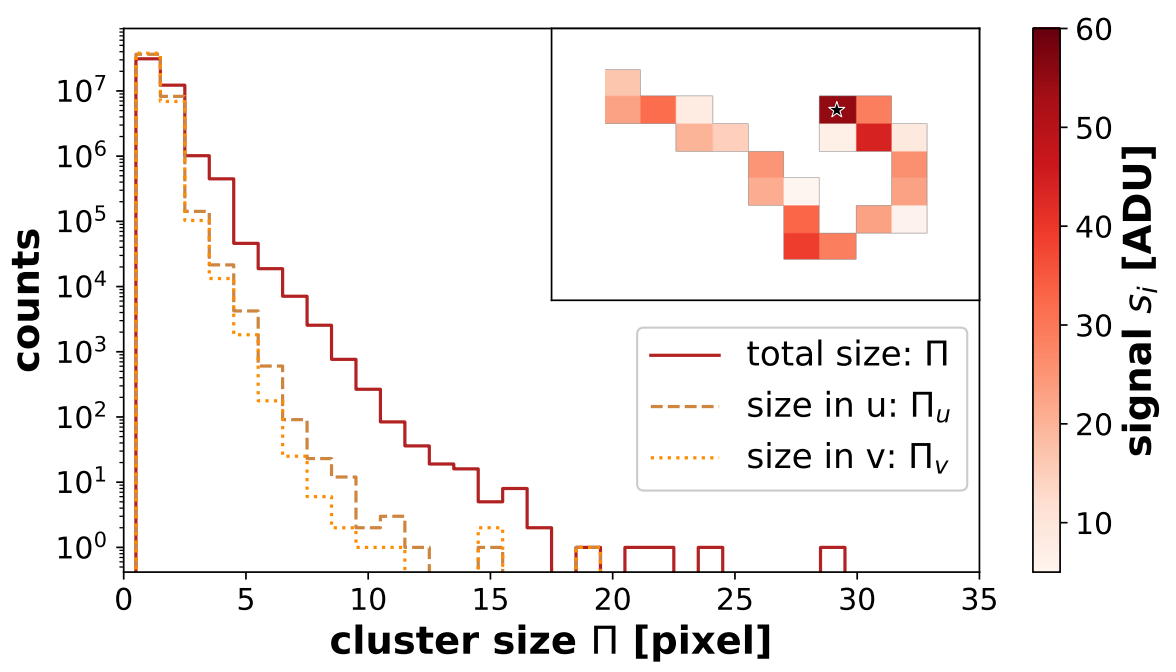

(b) cluster size and example of a large cluster caused by a $\delta$-electron

Figure 8.9.: Representative distributions of the calibrated total cluster energy $Q$ and of the cluster size $\Pi$ at perpendicular incidence measured for module W40_IF. An example of a $\delta$-electron cluster of size 21 is shown with the absolute pixel signals in units of ADU indicated by the colour scale. The extrapolated track intersection coordinate is indicated by the star marker. 


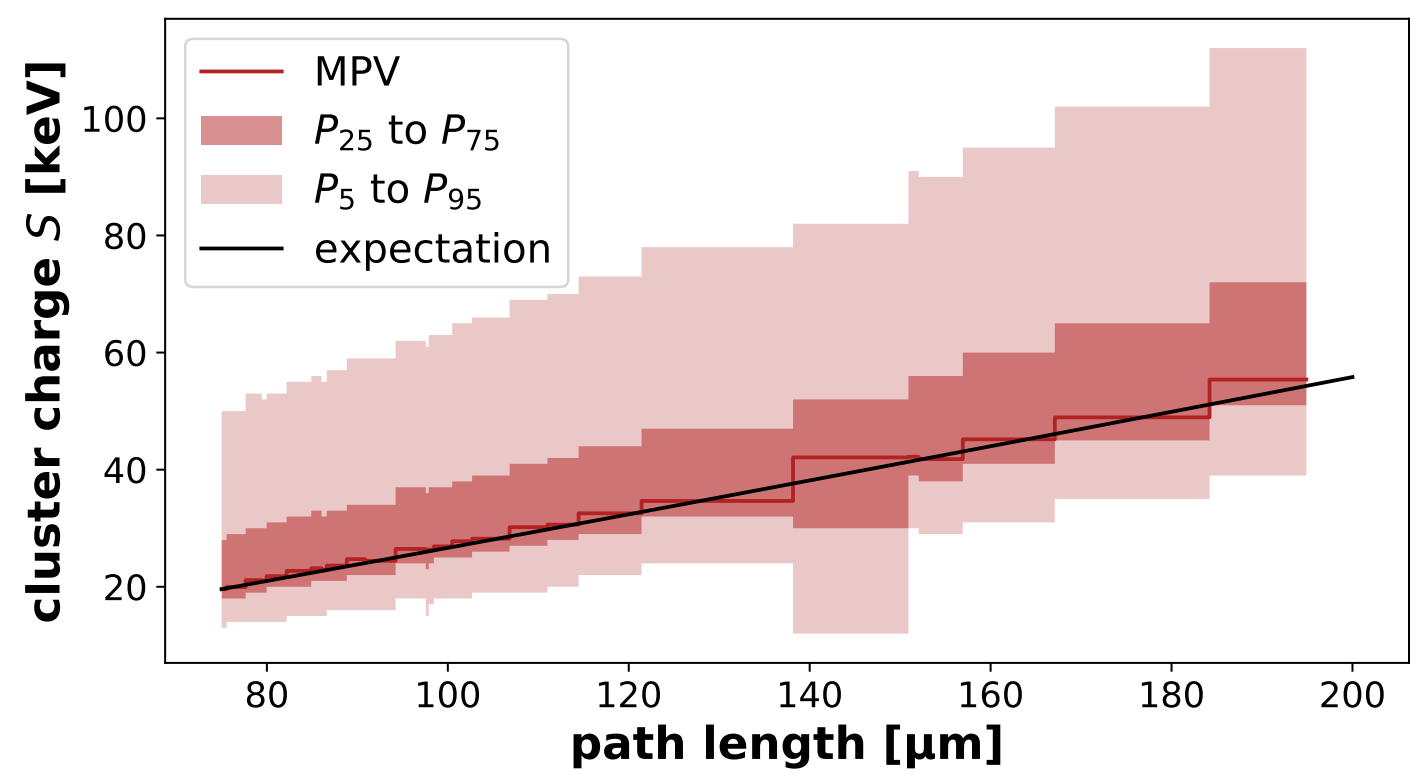

Figure 8.10.: The cluster charge distribution (outlined by the MPV and the percentiles) as a function of the path length of the electron track in the sensitive volume of the $75 \mu \mathrm{m}$ thick sensor computed by Eq. 8.16 and the expectation for the MPV of the distribution computed by Eq. 8.17.

$\Delta_{p} / x$, also scales with the total path length such that the expected cluster charge MPV, $Q_{\mathrm{MPV}}^{\mathrm{pred}}$ as a function of the incidence angles is

$$
Q_{\mathrm{MPV}}^{\mathrm{pred}}(\phi, \theta)=Q_{\mathrm{MPV}}(0,0) \frac{\Delta_{p}(l(\phi, \theta), \beta, \gamma)}{\Delta_{p}\left(l_{0}, \beta, \gamma\right)},
$$

where $\beta$ and $\gamma$ are the relativistic parameters of the impinging electron and the material dependent function $\Delta_{p}$ is given in Equation 34.12 in [16]. The reference value, $Q_{\mathrm{MPV}}(0,0)$, is the measured $Q_{\mathrm{MPV}}$ at perpendicular incidence. Only clusters matched to a reconstructed track are considered. The incidence angles $\phi$ and $\theta$ are determined as the average of the respective predicted intersection angles $\phi^{\text {pred }}$ and $\theta^{\text {pred }}$ from the track fit over all clusters measured at the respective DUT rotation. Figure 8.10 compares the expected $Q_{\mathrm{MPV}}^{\mathrm{pred}}$ to the measured $Q_{\mathrm{MPV}}$ as a function of the total path length of the electrons in the sensitive volume. Measurement and expectation are in good agreement. The comparison can also be done for the individual angles as shown in Figure 8.11. Due to the increased charge sharing at inclined particle incidence, larger clusters are expected to be measured. Figure 8.12 shows the average total cluster size $\langle\Pi\rangle$ as a function of the incidence angles. The data points are split into two groups corresponding to clusters formed by pixels of only one of the two pixel pitches. The average cluster size increases for increasing (more parallel to the sensor surface) incidence angles. The smaller pixel pitch results in larger clusters on average. 


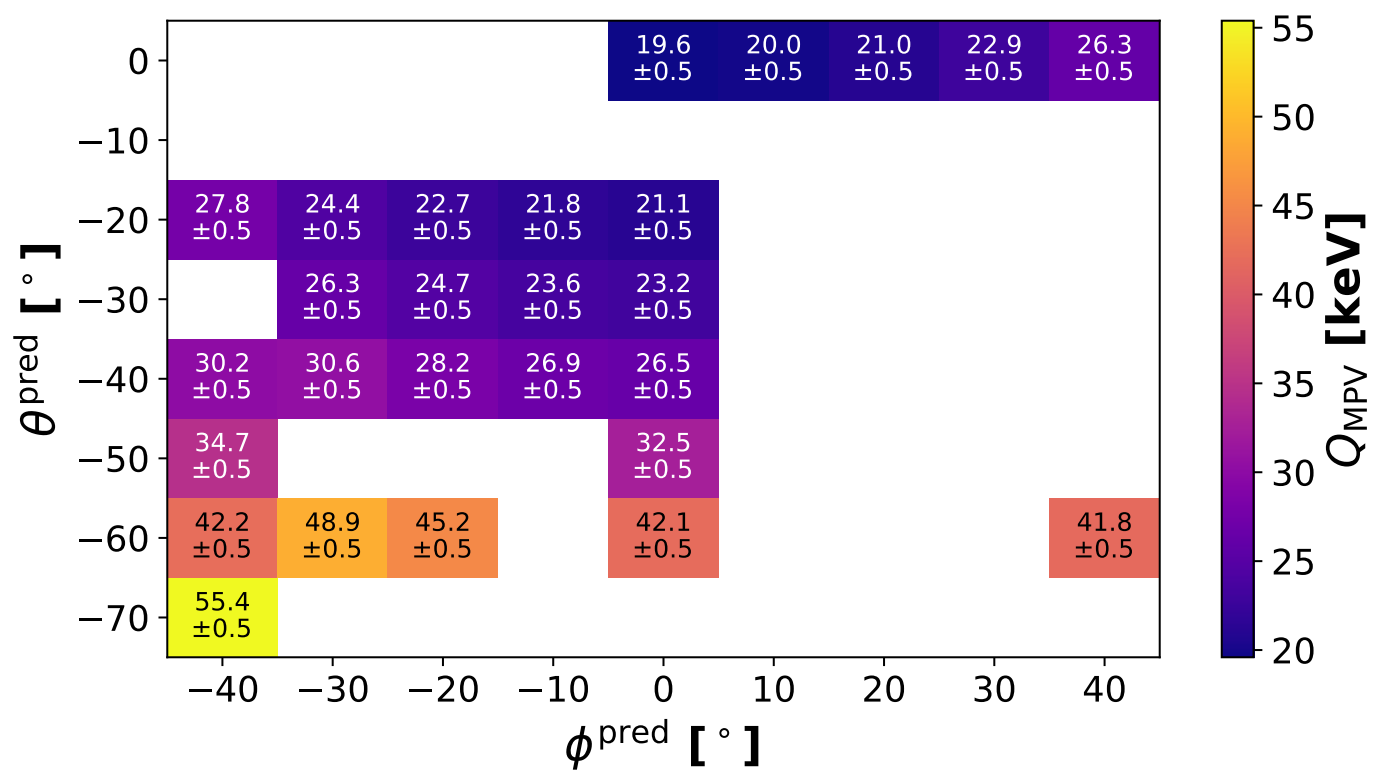

(a) pixel pitch $P_{v}=55 \mu \mathrm{m}$ of module W40_IF

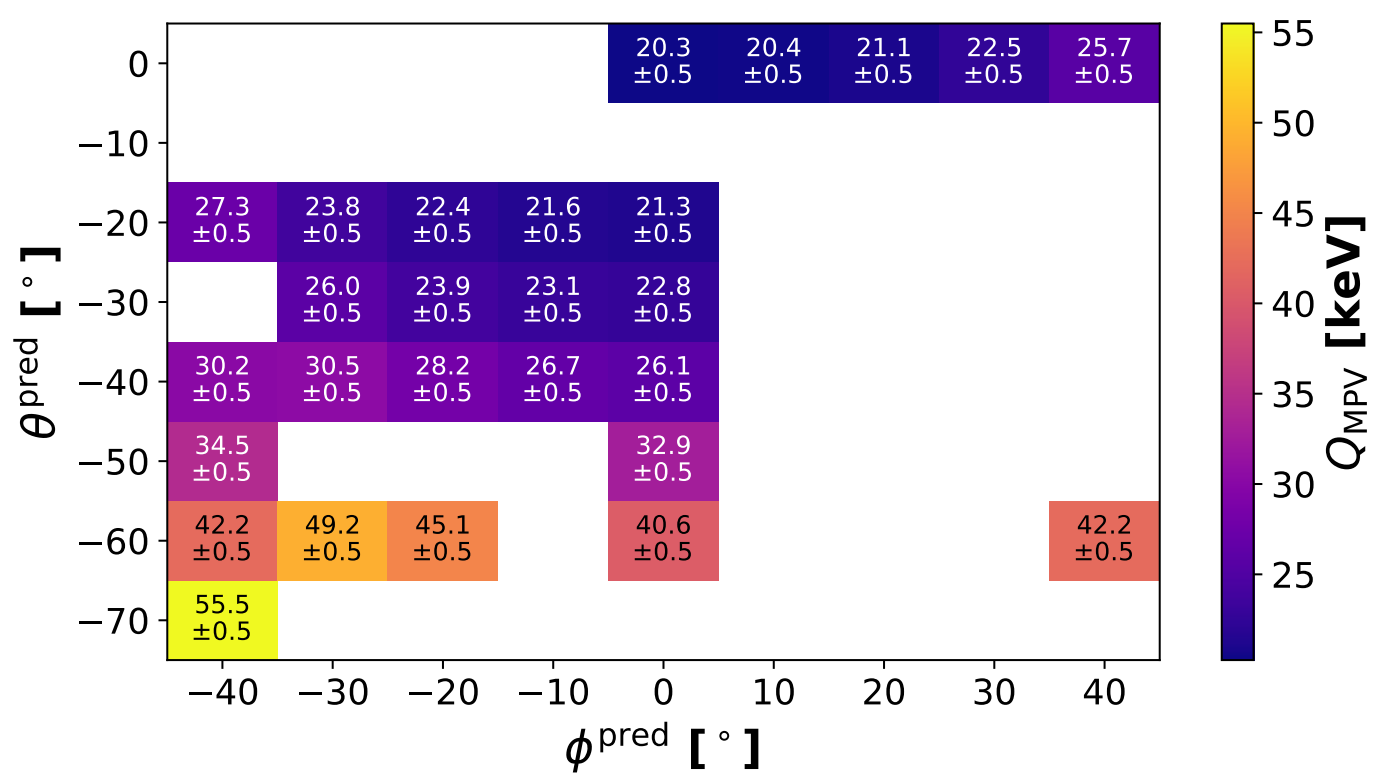

(b) pixel pitch $P_{v}=60 \mu \mathrm{m}$ of module W40_IF

Figure 8.11.: Most probable value $Q_{\mathrm{MPV}}$ of the total cluster energy distribution as a function of the predicted incidence angles for the two pixel pitches of module W40_IF. The MPV is determined by a LanGau fit to the cluster charge distribution taken over all pixels of the respective pitch. 


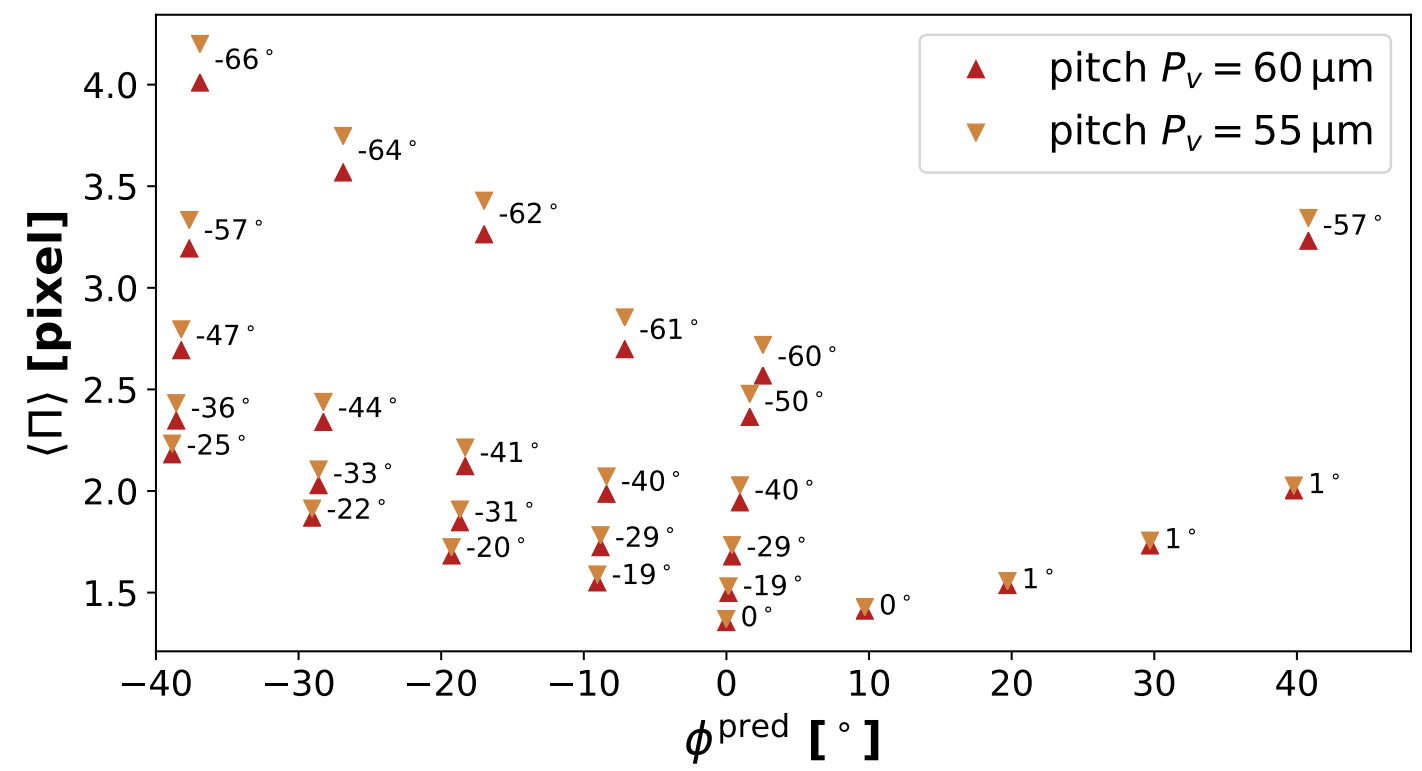

Figure 8.12.: Average total cluster size $\langle\Pi\rangle$ as a function of the predicted incidence angles, separated in the different pixel pitches of module W40_IF. The numbers on the right of the measurement points indicate the respective value of $\theta^{\text {pred }}$. 


\subsection{Resolution studies}

The track impact parameter resolution of Belle II significantly depends on the spatial resolution of the innermost layers of the vertex detector [17]. Measuring the intrinsic spatial resolution of the modules constituting the Belle II pixel detector, especially at inclined particle incidence, is thus crucial for understanding the performance of the vertex detector. Perpendicular particle incidence was measured on all DUTs. A detailed measurement of inclined particle incidence was performed with module W40_IF, a module for the innermost layer of the PXD. The spatial resolution is determined as a function of pixel pitch, particle incidence inclination and employed position finding algorithm (PFA). The following sections discuss how residual distributions and the telescope pointing resolution are extracted and used to determine the intrinsic spatial resolution of the DUTs and present the results for the different scenarios.

\subsubsection{Residuals and pointing resolution}

The residual $r$ (cf. Eq. 8.11) on the DUT plane measures the difference (distance) between a particle's impinging coordinates $(\hat{u}, \hat{v})$ on the DUT, extracted from the cluster by the PFA, and the extrapolated intersection coordinate $\left(u^{\text {pred }}, v^{\text {pred }}\right)$, given by the associated reconstructed track. The corresponding residual covariance matrix $V_{r}$ can be calculated as

$$
V_{r}=\left(\begin{array}{cc}
\operatorname{Var}\left(\hat{u}-u^{\text {pred }}\right) & \operatorname{Cov}\left(\hat{u}-u^{\text {pred }}, \hat{v}-v^{\text {pred }}\right) \\
\operatorname{Cov}\left(\hat{v}-v^{\text {pred }}, \hat{u}-u^{\text {pred }}\right) & \operatorname{Var}\left(\hat{v}-v^{\text {pred }}\right)
\end{array}\right)
$$

where the variance ${ }^{6}$ (Var) and covariance ${ }^{7}$ (Cov) of the residual distributions are computed over a set of tracks. The matrix $V_{r}$ can be decomposed as

$$
V_{r}=V^{\mathrm{DUT}}+T
$$

in a contribution from the covariance matrix of the intrinsic spatial resolution of the DUT, $V^{\text {DUT }}$, and the covariance matrix of the pointing resolution $T$ (cf. Eq. 8.12). The matrix $T$ is computed as the average of the extrapolation covariance matrices over the track set. The pointing resolution in $u$-direction, $\sigma_{u}^{\text {tel }}$, and in $v$-direction, $\sigma_{v}^{\text {tel }}$, are given as the square root of the diagonal elements of $T$. The statistical uncertainty on $\sigma_{u}^{\text {tel }}$ and $\sigma_{v}^{\text {tel }}$ are determined as the statistical uncertainty on the average. Besides the telescope geometry, the pointing resolution strongly depends on the resolution of the detector planes of the beam telescope, as well as on the beam energy. A systematic uncertainty on the pointing resolution is determined by repeating the track reconstruction and separately varying the assumed beam energy by $\pm 5 \%$ (cf. Fig. 8.2) and the effective MIMOSA26 intrinsic resolution by $\pm 5 \%$. Table 8.5 lists the extracted pointing resolution for the various beam energies and incidence angles. The best, that means smallest, pointing resolution is obtained at perpendicular incidence $\left(\phi=0^{\circ}, \theta=0^{\circ}\right)$ for

\footnotetext{
${ }^{6}$ The variance of a random variable $x$ is $\operatorname{Var}(x)=\left\langle(x-\langle x\rangle)^{2}\right\rangle$.

${ }^{7}$ The covariance of two random variables $x$ and $y$ is $\operatorname{Cov}(x, y)=\langle(x-\langle x\rangle)(y-\langle y\rangle)\rangle$.
} 
a beam energy of $E_{\text {beam }}=5 \mathrm{GeV}$, yielding $\sigma^{\text {tel }}=3.3 \pm 0.2$ (stat.) \pm 0.3 (syst.) $\mu \mathrm{m}$ in both $u$ - and $v$-direction. The pointing resolution becomes worse for increasing rotation angles, up to $\sigma_{u}^{\text {tel }}=8.7 \pm 0.1$ (stat.) \pm 1.0 (syst.) $\mu \mathrm{m}$ and $\sigma_{v}^{\text {tel }}=17.4 \pm 0.1$ (stat.) \pm 2.2 (syst.) $\mu \mathrm{m}$ for incidence angles of $\phi=39^{\circ}$ and $\theta=66^{\circ}$. Note that for increasing DUT rotation angles, also the distances between detector planes had to be adjusted, which influence the pointing resolution (cf. Tab. 8.1). While the statistical uncertainty is rather constant at about $0.2 \mu \mathrm{m}$ over all measured incidence angles, the systematic uncertainty increases significantly for large incidence angles. The increase at large angles is due to the different telescope geometry with larger distances between the telescope arms and in between the MIMOSA26 planes.

The distribution of the residuals $\hat{u}-u^{\text {pred }}$ and $\hat{v}-v^{\text {pred }}$, respectively, are expected to be unbiased, that means centred at zero. The square root of the variance in $V_{r}$ are estimated as the root-mean-square (RMS) of the residual distributions in $u$ - and $v$-direction, respectively. That is

$$
\sqrt{\operatorname{Var}\left(\hat{u}-u^{\text {pred }}\right)}=\sqrt{\left\langle\left(\hat{u}-u^{\text {pred }}\right)^{2}\right\rangle}
$$

and analogously in $v$-direction. The RMS is computed over the range of $-100 \mu \mathrm{m}$ to $100 \mu \mathrm{m}$ to exclude the tails in the residual distribution that are due to single events with large multiple scattering angles. An overall systematic and constant bias of the residual distributions would be corrected for during the alignment process. It is, however, observed in some experiments, that the bias (i.e. the centre) of the residual distribution drifts over time. Figure 8.13 shows an example of the bias drift of one experiment. It depicts a two-dimensional histogram of the residual in $u$-direction and the run number, which is roughly increasing evenly over time. There seems to be an initial, strong bias drift that saturates over time (from smaller to larger run numbers). Since the calibration and thus the alignment is done using the final five runs of the experiment ${ }^{8}$, the residuals distributions are centred for the highest run numbers. This effect was only observed after the beam tests were finished, the origin is unclear. The bias drift is negligibly small for measurements at perpendicular incidence for all DUTs. The most likely explanation is a physical movement over time, for example by an insufficient fixation of the motor stages. The influence of the bias drift was mitigated by subtracting the bias of the distribution for every group of $10^{5}$ events. The difference $\delta_{\text {bias }}^{i}$ of the bias correction of consecutive event groups $i$ and $i+1$ approximates the remaining bias drift within the event group. This remaining drift broadens the overall width of the residual distribution. Assuming that the contribution of the drift to the total RMS can be described by a box distribution of width $\left\langle\delta_{\text {bias }}\right\rangle$, which is the average over all $\delta_{\text {bias }}^{i}$ within one experiment, it can be quantified ${ }^{9}$ as $\left\langle\delta_{\text {bias }}\right\rangle / \sqrt{12}$. This contribution is in the order of $0.1 \mu \mathrm{m}$ and is interpreted

\footnotetext{
${ }^{8}$ This procedure was established in response to the observed bias drift behaviour. Using the final runs ensures that the alignment is performed under a stable geometrical condition, where the drift has saturated. The data of five final runs is used to have a sufficiently large data set for a reliable alignment procedure.

${ }^{9}$ The variance of a centred and symmetric continuous uniform distribution, or simply box distribution, is given by width ${ }^{2} / 12$.
} 
8. Measuring Pixel Detector Modules in Beam Tests

\begin{tabular}{rr|ccc}
\hline$\phi^{\text {pred }}\left[{ }^{\circ}\right]$ & $\theta^{\text {pred }}\left[{ }^{\circ}\right]$ & $E_{\text {beam }}[\mathrm{GeV}]$ & $\sigma_{u}^{\text {tel }} \pm($ stat. $) \pm($ syst.) $[\mu \mathrm{m}]$ & $\sigma_{v}^{\text {tel }} \pm($ stat. $) \pm($ syst. $)[\mu \mathrm{m}]$ \\
\hline$-38.9(1)$ & $-24.5(1)$ & $5.0(1)$ & $6.0 \pm 0.2 \pm 0.4$ & $5.1 \pm 0.2 \pm 0.4$ \\
$-38.6(1)$ & $-36.2(1)$ & $5.0(1)$ & $6.7 \pm 0.2 \pm 0.5$ & $6.4 \pm 0.2 \pm 0.5$ \\
$-38.2(1)$ & $-47.3(1)$ & $5.0(1)$ & $7.4 \pm 0.1 \pm 0.6$ & $8.6 \pm 0.1 \pm 0.7$ \\
$-37.7(1)$ & $-57.5(1)$ & $5.0(1)$ & $8.1 \pm 0.1 \pm 0.8$ & $11.9 \pm 0.1 \pm 1.1$ \\
$-36.9(1)$ & $-66.3(1)$ & $5.0(1)$ & $8.7 \pm 0.1 \pm 1.0$ & $17.4 \pm 0.1 \pm 2.2$ \\
\hline$-29.0(1)$ & $-21.9(1)$ & $5.0(1)$ & $4.9 \pm 0.2 \pm 0.4$ & $4.6 \pm 0.2 \pm 0.4$ \\
$-28.6(1)$ & $-32.8(1)$ & $5.0(1)$ & $5.6 \pm 0.2 \pm 0.4$ & $5.8 \pm 0.2 \pm 0.5$ \\
$-28.3(1)$ & $-43.8(1)$ & $5.0(1)$ & $6.2 \pm 0.2 \pm 0.5$ & $7.6 \pm 0.2 \pm 0.6$ \\
$-26.9(1)$ & $-63.7(1)$ & $5.0(1)$ & $7.7 \pm 0.1 \pm 0.9$ & $15.4 \pm 0.1 \pm 1.8$ \\
\hline$-19.3(1)$ & $-20.2(1)$ & $5.0(1)$ & $4.2 \pm 0.2 \pm 0.3$ & $4.3 \pm 0.2 \pm 0.3$ \\
$-18.7(1)$ & $-30.7(1)$ & $5.0(1)$ & $4.8 \pm 0.2 \pm 0.4$ & $5.2 \pm 0.2 \pm 0.4$ \\
$-18.3(1)$ & $-41.5(1)$ & $5.0(1)$ & $5.5 \pm 0.2 \pm 0.4$ & $6.9 \pm 0.2 \pm 0.5$ \\
$-17.0(1)$ & $-61.9(1)$ & $5.0(1)$ & $7.0 \pm 0.1 \pm 0.8$ & $14.2 \pm 0.1 \pm 1.6$ \\
\hline$-9.1(1)$ & $-19.1(1)$ & $5.0(1)$ & $3.7 \pm 0.2 \pm 0.3$ & $3.9 \pm 0.2 \pm 0.3$ \\
$-8.8(1)$ & $-29.4(1)$ & $5.0(1)$ & $4.2 \pm 0.2 \pm 0.3$ & $4.8 \pm 0.2 \pm 0.4$ \\
$-8.4(1)$ & $-40.2(1)$ & $5.0(1)$ & $5.0 \pm 0.2 \pm 0.4$ & $6.4 \pm 0.2 \pm 0.5$ \\
$-7.1(1)$ & $-60.6(1)$ & $5.0(1)$ & $7.1 \pm 0.1 \pm 1.1$ & $14.4 \pm 0.1 \pm 2.2$ \\
\hline $0.0(1)$ & $0.0(1)$ & $3.0(2)$ & $3.8 \pm 0.2 \pm 0.3$ & $3.8 \pm 0.2 \pm 0.2$ \\
\hline $0.0(1)$ & $0.0(1)$ & $5.0(1)$ & $3.3 \pm 0.2 \pm 0.3$ & $3.3 \pm 0.2 \pm 0.2$ \\
$0.1(1)$ & $-18.8(1)$ & $5.0(1)$ & $3.5 \pm 0.2 \pm 0.3$ & $3.7 \pm 0.2 \pm 0.3$ \\
$0.4(1)$ & $-29.0(1)$ & $5.0(1)$ & $4.1 \pm 0.2 \pm 0.3$ & $4.6 \pm 0.2 \pm 0.4$ \\
$1.1(1)$ & $-39.7(1)$ & $5.0(1)$ & $4.8 \pm 0.2 \pm 0.4$ & $6.2 \pm 0.2 \pm 0.5$ \\
$1.6(1)$ & $-50.3(1)$ & $5.0(1)$ & $5.6 \pm 0.2 \pm 0.6$ & $8.7 \pm 0.1 \pm 0.9$ \\
$2.6(1)$ & $-60.2(1)$ & $5.0(1)$ & $8.3 \pm 0.1 \pm 1.1$ & $16.6 \pm 0.1 \pm 2.1$ \\
\hline $9.7(1)$ & $0.4(1)$ & $5.0(1)$ & $3.3 \pm 0.2 \pm 0.3$ & $3.3 \pm 0.2 \pm 0.2$ \\
$19.7(1)$ & $0.6(1)$ & $5.0(1)$ & $3.9 \pm 0.2 \pm 0.3$ & $3.6 \pm 0.2 \pm 0.3$ \\
$29.7(1)$ & $0.9(1)$ & $5.0(1)$ & $4.7 \pm 0.2 \pm 0.3$ & $4.0 \pm 0.2 \pm 0.3$ \\
$39.8(1)$ & $0.9(1)$ & $5.0(1)$ & $5.9 \pm 0.2 \pm 0.4$ & $12.4 \pm 0.1 \pm 1.1$ \\
\hline $40.8(1)$ & $-57.3(1)$ & $5.0(1)$ & $8.9 \pm 0.1 \pm 0.8$ & \\
\hline & & & & \\
\hline & & & & \\
\hline
\end{tabular}

Table 8.5.: Pointing resolution on the DUT plane in $u$-and $v$-direction per incidence angles and beam energy. The incidence angles are given as the predicted incidence angle, averaged over all reconstructed tracks. The uncertainties on the pointing resolution are given in a statistical contribution and a systematic contribution, as discussed in the main text. The rows are separated in groups of identical nominal incidence angle $\phi$ and beam energy $E_{\text {beam }}$. 
as a systematic uncertainty on the RMS of the residual distribution. Figure 8.14 shows the overall distribution of residuals in $u$-direction (same data set as used in Fig. 8.13) with and without the bias drift correction applied.

With both the RMS of the residual distribution (diagonal elements of $V_{r}$ ) and the pointing resolution (diagonal elements of $T$ ) at hand, the intrinsic spatial resolution (diagonal elements of $V^{\text {DUT }}$ ) can be computed according to Eq. 8.19. Specifically, the intrinsic resolution of the DUT is computed as

$$
\sigma_{u}^{\mathrm{DUT}}=\sqrt{\operatorname{Var}\left(\hat{u}-u^{\mathrm{pred}}\right)-\left(\sigma_{u}^{\mathrm{tel}}\right)^{2}}
$$

and analogously in $v$-direction. The statistical and systematic uncertainties of the RMS and the pointing resolution are propagated through Eq. 8.21 to obtain the statistical and systematic uncertainty on $\sigma_{u}^{\text {DUT }}$ and $\sigma_{v}^{\text {DUT }}$, respectively. The intrinsic resolution in $v$-direction can be considered separately for the two different pixel pitches $P_{v}$ on each DUT. It is obvious that the uncertainty on the intrinsic resolution becomes very dominant when the RMS and the pointing resolution are of comparable size. This is not surprising since in that case the resolution of the tool (i.e. the pointing resolution) used to probe the quantity (i.e. the intrinsic resolution) is of the same size as the quantity itself, providing only coarse information. 


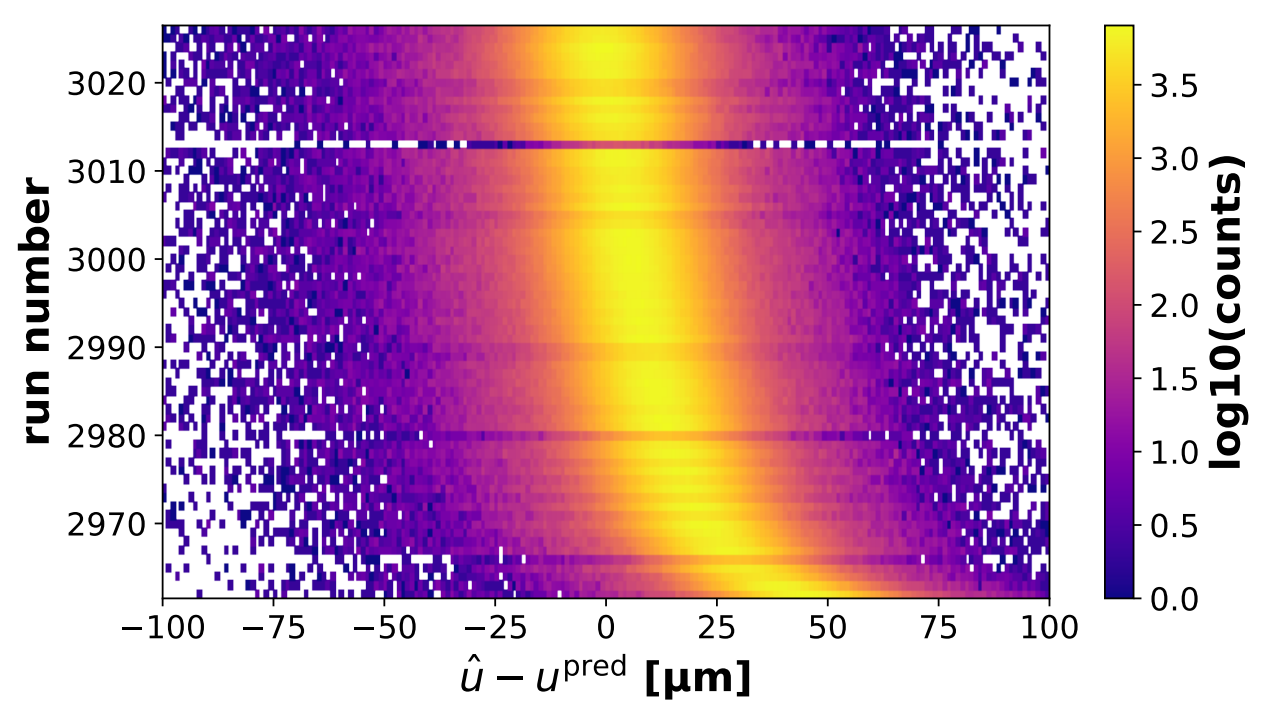

Figure 8.13.: Two-dimensional histogram of the (not bias corrected) residuals in $u$ direction and the run number for one experiment (incidence at $\phi=40^{\circ}$ and $\theta=20^{\circ}$ ) of the beam test in 2018. The colour scale indicates the 10 th logarithm of the number of the entries per bin. Run 3013 collects only a small number of events.

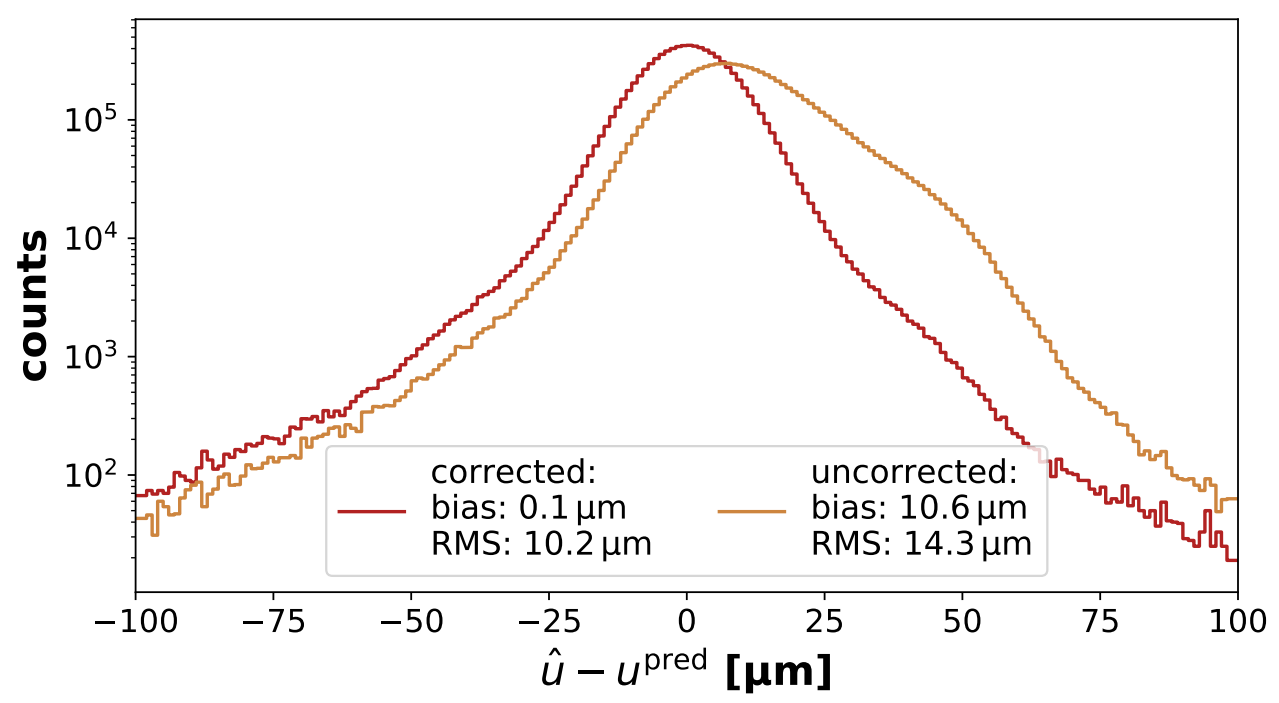

Figure 8.14.: Distribution of residuals in $u$-direction of one experiment with a significant bias drift (cf. Fig. 8.13) with and without the bias correction applied. The asymmetry, as well as the overall offset of the uncorrected distribution is due to a drifting bias over time. 


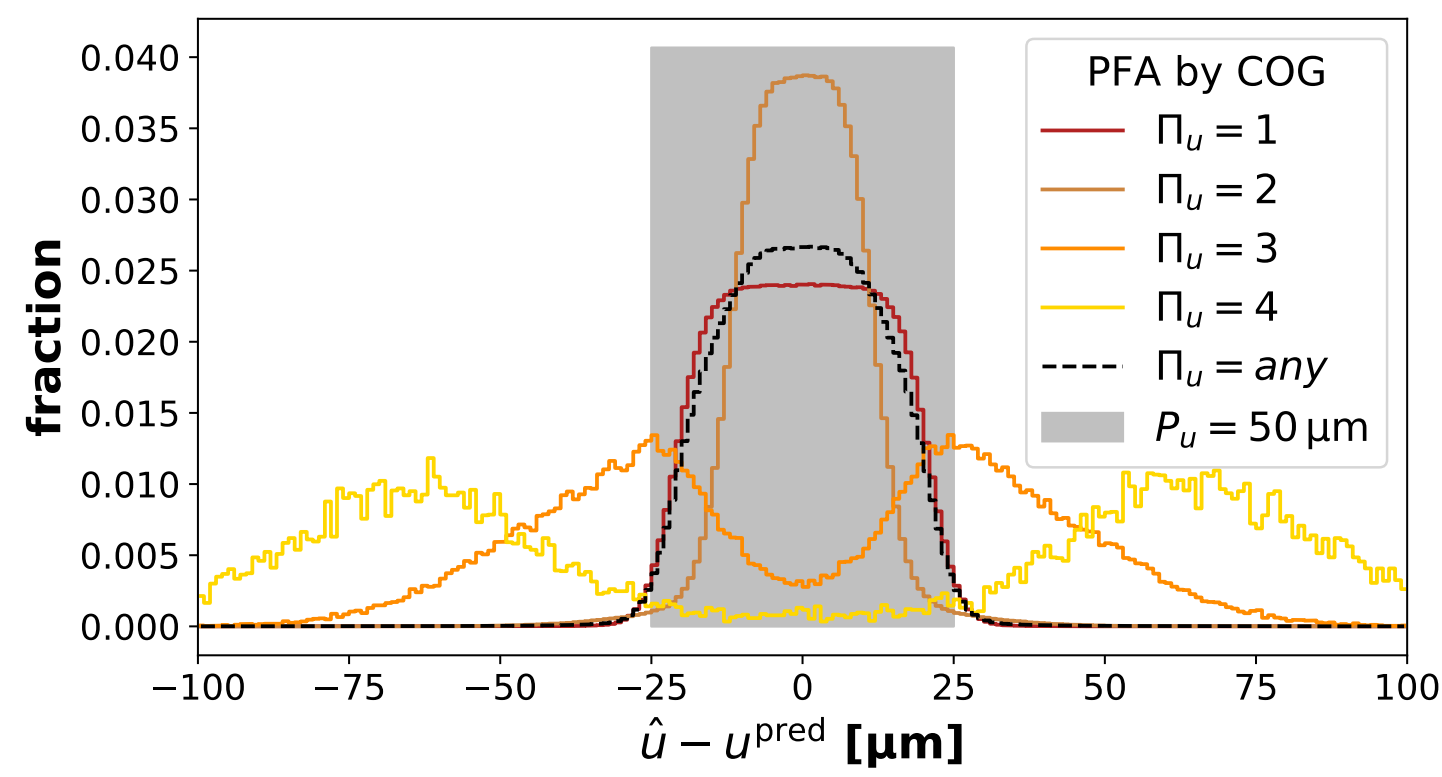

Figure 8.15.: Residual distribution in $u$-direction as a function of the cluster size in $u$ direction $\left(\Pi_{u}\right)$ for perpendicular particle incidence using the COG PFA. The dashed line $\left(P_{u}=\right.$ any) indicates the residual distribution including all cluster sizes. The grey box indicates the geometrical dimension, the pixel pitch $P_{u}$, of a single pixel in $u$-direction.

\subsubsection{Intrinsic spatial resolution at perpendicular incidence}

The intrinsic spatial resolution at perpendicular incidence was extracted from the measurements of modules W40_IF and W11_OF2. Both modules were measured in the same beam test in 2018 and thus provide best comparability regarding the experimental conditions. All four different pixel pitches in $v$-direction are covered by this selection. To start with, the spatial resolution based on residuals obtained with the centre of gravity (COG) PFA is discussed.

Figure 8.15 shows the distribution of the residuals in $u$-direction as a function of the cluster size in $u$-direction $\left(\Pi_{u}\right)$. The width of the distribution decreases - and thus the intrinsic spatial resolution improves, as will be shown - for two-pixel clusters $\left(\Pi_{u}=2\right)$ compared to one-pixel clusters $\left(\Pi_{u}=1\right)$. For larger cluster sizes, the overall width increases again. The intrinsic spatial resolution extracted from these residual distributions for all pixel pitches in $u$ - and $v$-direction are listed in Table 8.6.

For one-pixel clusters $\left(\Pi_{u}=\Pi_{v}=\Pi=1\right)$, no charge is shared between pixels (or the information is lost due to threshold effects or non-functional pixel neighbours). The position where the impinging particle traversed the pixel has to be estimated with a uniform probability distribution over the pixel area. This approach yields the $P / \sqrt{12}$ binary resolution, where $P$ is the pixel pitch in the respective direction. However, the 


\begin{tabular}{c|cc|c}
\hline pitch $P_{u}[\mu \mathrm{m}]$ & $\Pi_{u}[$ pixel] & fraction [\%] & $\sigma_{u}^{\text {DUT }} \pm$ (stat.) \pm (syst.) $[\mu \mathrm{m}]$ \\
\hline 50 & 1 & 81.2 & $12.14 \pm 0.1 \pm 0.3$ \\
& 2 & 18.4 & $10.16 \pm 0.1 \pm 0.3$ \\
& 3 & 0.31 & $33.91 \pm 0.1 \pm 0.3$ \\
& 4 & 0.05 & $64.36 \pm 0.2 \pm 0.3$ \\
\hline pitch $P_{v}[\mu \mathrm{m}]$ & $\Pi_{v}[$ pixel $]$ & fraction [\%] & $\sigma_{v}^{\text {DUT }} \pm$ (stat.) $\pm($ syst.) $[\mu \mathrm{m}]$ \\
\hline 55 & 1 & 68.1 & $13.91 \pm 0.1 \pm 0.1$ \\
& 2 & 28.1 & $10.74 \pm 0.1 \pm 0.1$ \\
& 3 & 2.5 & $41.14 \pm 0.1 \pm 0.04$ \\
& 4 & 1.06 & $75.07 \pm 0.5 \pm 0.03$ \\
& $\geq 5$ & 0.24 & $119.84 \pm 1.1 \pm 0.03$ \\
\hline 60 & 1 & 69.4 & $15.36 \pm 0.1 \pm 0.1$ \\
& 2 & 27.2 & $11.56 \pm 0.1 \pm 0.1$ \\
& 3 & 2.3 & $45.60 \pm 0.1 \pm 0.04$ \\
& 4 & 0.95 & $82.87 \pm 0.5 \pm 0.03$ \\
& $\geq 5$ & 0.15 & $127.58 \pm 1.1 \pm 0.03$ \\
\hline 70 & 1 & 60.7 & $18.09 \pm 0.1 \pm 0.1$ \\
& 2 & 33.0 & $14.13 \pm 0.1 \pm 0.1$ \\
& 3 & 3.9 & $46.34 \pm 0.1 \pm 0.1$ \\
& 4 & 2.3 & $89.56 \pm 0.6 \pm 0.1$ \\
& $\geq 5$ & 0.1 & $137.31 \pm 1.5 \pm 0.1$ \\
\hline 85 & 1 & 60.0 & $20.83 \pm 0.1 \pm 0.1$ \\
& 2 & 33.4 & $17.12 \pm 0.1 \pm 0.1$ \\
& 3 & 4.0 & $56.07 \pm 0.2 \pm 0.1$ \\
& 4 & 2.5 & $106.87 \pm 0.9 \pm 0.1$ \\
& $\geq 5$ & 0.1 & $152.01 \pm 2.6 \pm 0.1$ \\
\hline & & &
\end{tabular}

Table 8.6.: Intrinsic spatial resolution in $u$-direction ( $v$-direction) dependent on the pixel pitch $P_{u}\left(P_{v}\right)$ and the cluster size $\Pi_{u}\left(\Pi_{v}\right)$ for perpendicular particle incidence using the COG PFA. The cluster sizes are given with their respective fraction in the data set. The uncertainty on the intrinsic spatial resolution is given with the statistical and systematic contribution. 
effect of charge sharing can be employed indirectly. Since particles traversing pixels close to the borders or edges between pixels are very likely to cause multi-pixel clusters, a charge sharing region around the pixel edges can be defined. One-pixel clusters are largely only measured if the particle traversed a pixel in the central region. Thus, when considering one-pixel clusters specifically, an effective pixel pitch $P_{\text {eff }}<P$ of this central region has to be adopted. The effective pitch also depends on the incidence angle of the traversing particle.

Multi-pixel clusters on the other hand are likely measured when an impinging particle traverses a pixel in the charge sharing region. This information can be used to improve the precision of the estimation of the the impinging coordinate. Figure 8.15 confirms that in the case of a cluster size of $\Pi_{u}=2$, the residual distribution is more narrow. Table 8.6 indeed proves that the intrinsic spatial resolution for $\Pi_{u / v}=2$ is better (i.e. smaller) than for one-pixel clusters at identical pixel pitch.

The COG PFA assumes a linear charge sharing. For a cluster of size $\Pi=2$ with a large asymmetry in the pixel signals $s_{1} \gg s_{2}$, the impinging coordinate estimated by the COG PFA is nearly identical to the centre of pixel 1. However, charge sharing mostly happens in the charge sharing region and not in the centre. This non-linearity is respected in the cluster shape PFA (CSA). The ratio $\rho$ of the two pixel signals (or in general the ratio of the head and tail pixel signals, cf. Eq. 8.6) is binned and the combination of cluster shape and $\rho$-bin yields the estimate of the impinging coordinate. There is no assumption of a linear dependence between these quantities in the CSA. The COG PFA is a special case of the CSA. Especially in the case of perpendicular particle incidence, the charge sharing region is expected to be small and the CSA can yield better estimates compared to the COG PFA.

The CSA was calibrated requesting $N_{\rho}=14 \rho$-bins and requiring $N_{\mathcal{C}} \geq 100$ clusters per $\rho$-S-bin. In total $N_{\mathcal{S}}=25$ distinct cluster shapes and and overall $113 \rho$-S-bins are present in the cluster database, covering $99.5 \%$ of all clusters of the calibration sample. The distribution of the residuals in $u$-direction as a function of cluster size $\Pi_{u}$ based on the CSA are shown in Figure 8.16. In Figure 8.17, the residual distributions in $u$-direction for one-pixel and two-pixel clusters for the COG PFA and the CSA are compared. The residual distributions of clusters with size $\Pi_{u}=1$ are very compatible, as expected, since neither algorithm can exploit the non-existent charge sharing. The residual distributions of $\Pi_{u}=2$ clusters, on the other hand, show a significantly more narrow width for the CSA compared to the COG PFA. The intrinsic resolutions extracted from these residual distributions are listed in Table 8.7. For one-pixel clusters, the intrinsic resolution obtained with the CSA are compatible to the values obtained using the COG PFA. This is expected from the residual distributions and explained by the non-existent charge sharing. With increasing pixel pitch, the intrinsic resolution increases as well. In fact, the ratio of intrinsic resolution to the pixel pitch is rather constant with about $25 \%$ (or about $85 \%$ with respect to the binary resolution $P / \sqrt{12}$ ). For clusters of size two in the respective direction, the CSA yields better (i.e. smaller) intrinsic resolution consistently for all pixel pitches. Improvements of about $20 \%$ to $40 \%$ when using the CSA compared to using the COG PFA are observed. For larger clusters $\left(\Pi_{u / v} \geq 3\right)$, 


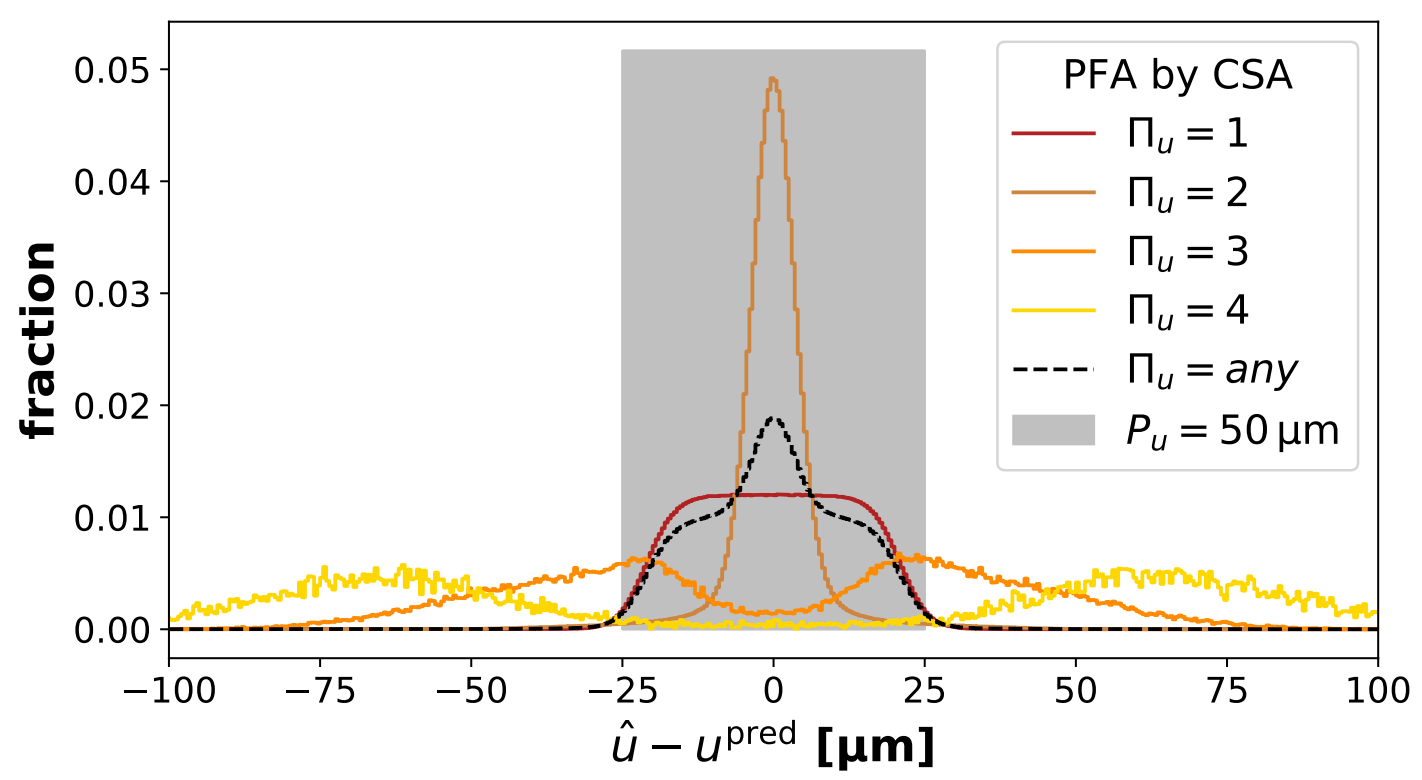

Figure 8.16.: Residual distribution in $u$-direction as a function of the cluster size in $u$ direction $\left(\Pi_{u}\right)$ for perpendicular particle incidence using the CSA. The dashed line $\left(P_{u}=\right.$ any) indicates the residual distribution including all cluster sizes. The grey box indicates the pixel pitch $P_{u}$.

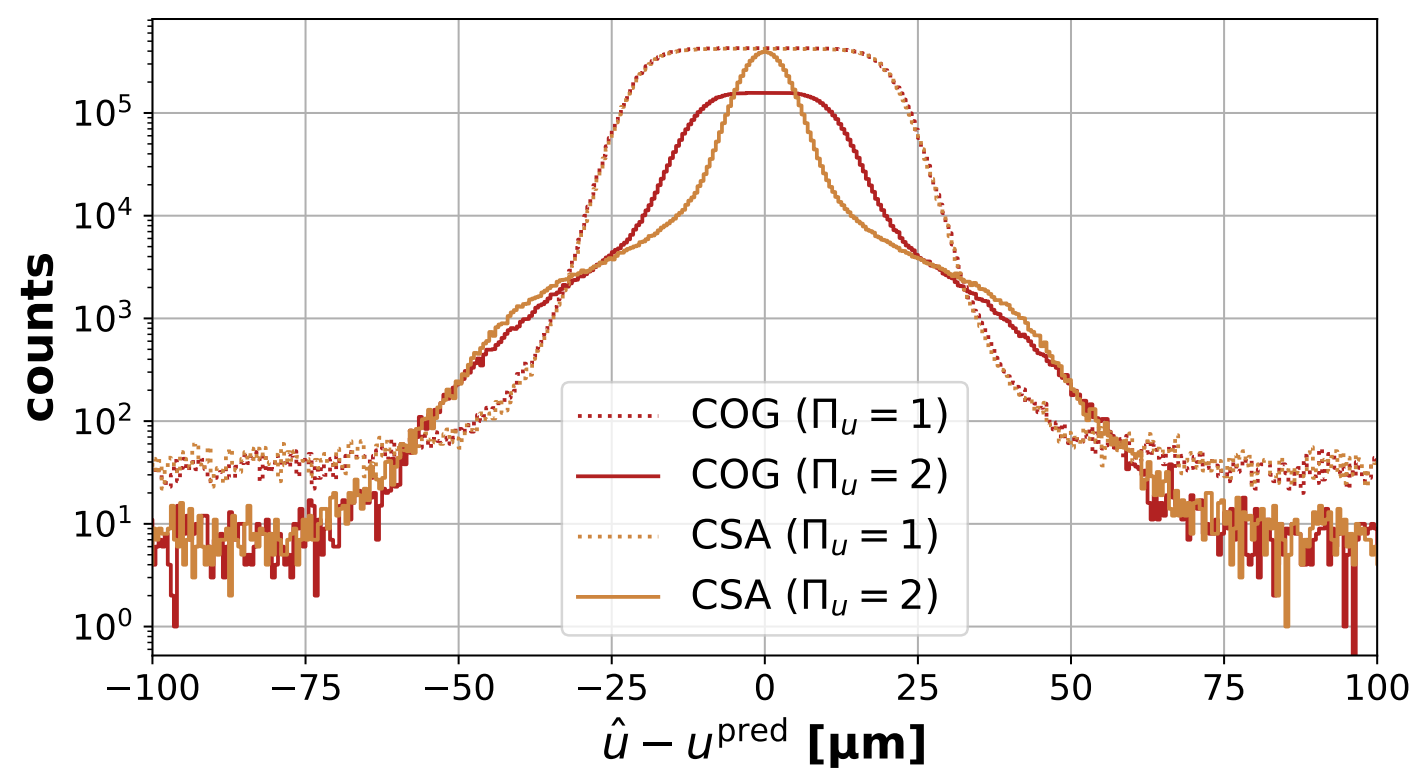

Figure 8.17.: Residual distribution in $u$-direction for one-pixel and two-pixel clusters for perpendicular particle incidence using the COG PFA and the CSA. 


\begin{tabular}{c|c|cc}
\hline & & \multicolumn{2}{|c}{$\sigma_{u}^{\text {DUT }}[\mu \mathrm{m}]$} \\
pitch $P_{u}[\mu \mathrm{m}]$ & $\Pi_{u}[\mathrm{pixel}]$ & COG PFA & CSA \\
\hline 50 & 1 & $12.1(3)$ & $12.2(3)$ \\
& 2 & $10.2(4)$ & $8.1(4)$ \\
& 3 & $33.9(3)$ & $34.3(3)$ \\
\hline \multirow{3}{*}{ pitch $P_{v}[\mu \mathrm{m}]$} & $\Pi_{v}[\mathrm{pixel}]$ & COG PFA & CSA \\
\hline 55 & 1 & $13.9(1)$ & $14.0(1)$ \\
& 2 & $10.7(1)$ & $7.7(2)$ \\
& 3 & $41.1(1)$ & $41.8(2)$ \\
\hline \multirow{2}{*}{60} & 1 & $15.4(1)$ & $15.4(1)$ \\
& 2 & $11.6(1)$ & $8.5(2)$ \\
& 3 & $45.6(1)$ & $46.0(1)$ \\
\hline 70 & 1 & $18.1(1)$ & $18.2(1)$ \\
& 2 & $14.1(1)$ & $8.4(2)$ \\
& 3 & $46.3(2)$ & $47.5(2)$ \\
\hline \multirow{2}{*}{85} & 1 & $20.8(1)$ & $20.9(1)$ \\
& 2 & $17.1(1)$ & $9.5(2)$ \\
& 3 & $56.1(2)$ & $57.6(2)$ \\
\hline
\end{tabular}

Table 8.7.: Intrinsic spatial resolution in $u$-direction ( $v$-direction) dependent on the pixel pitch $P_{u}\left(P_{v}\right)$ and the cluster size $\Pi_{u}\left(\Pi_{v}\right)$ for perpendicular particle incidence using the COG PFA and the CSA. The uncertainty on the intrinsic spatial resolution is given as the combined statistical and systematic contribution computed as the quadratic sum of the two. The systematic uncertainties are fully correlated between the COG PFA and the CSA.

there seems to be no performance gain over the COG PFA when using the CSA in the case of perpendicular particle incidence. This can be understood by considering the very small fraction of cluster of size $\geq 3$ (cf. Tab. 8.6). The statistics is insufficient for the CSA to be calibrated with an improved estimation compared to the COG PFA for these clusters.

\subsubsection{Inclined particle incidence}

Inclined particle incidence was measured with DUT W40_IF during the beam test in 2018. The inclination facilitates the effect of charge sharing since in contrast to perpendicular incidence, where the charge cloud is spread over neighbouring pixels by diffusion, the inclined trajectory itself spreads the charge cloud laterally. It is thus expected that the charge sharing region increases. The incidence angle $\theta$ was varied up to values of 


\section{Measuring Pixel Detector Modules in Beam Tests}

about $60^{\circ}$, close to the maximum acceptance of Belle II. For every combination of the incidence angles $\phi$ and $\theta$ that was measured, the CSA calibration is repeated with the hyperparameters chosen as $N_{\rho}=14$ (number of $\rho$-bins) and $N_{\mathcal{C}}=100$ (minimum number of clusters per $\rho$-S-bin). Table 8.8 lists the number of distinct cluster shapes, the overall number of $\rho$-S-bins found, the coverage and the number of clusters used as the calibration sample for the CSA per incidence angles. The number of distinct cluster shapes and $\rho$-S-bins increases for increasing incidence angles. As already observed in Figure 8.12, the average cluster size increases with increasing incidence angles resulting in more variations of cluster shapes. On average, $98.8 \%$ of the clusters of the CSA calibration sample are covered by the final lookup table. For most of the incidence angle combinations, the calibration sample comprises about $3 \times 10^{5}$ clusters $\left(P_{25}\left(n_{\mathcal{C}}\right)=2.81 \times 10^{5}\right)$.

For comparison, the residuals and resolutions per incidence angles were also computed using the COG PFA. Figure 8.18 shows the intrinsic spatial resolution $\sigma_{u}^{\text {DUT }}\left(\sigma_{v}^{\text {DUT }}\right)$ as a function of the absolute value of the predicted incidence angle $\phi^{\text {pred }}\left(\theta^{\text {pred }}\right)$. Measurement points of identical nominal incidence angle $\phi(\theta)$ are grouped by and the resolution is average over the variations of the respective perpendicular angle. The $\phi^{\text {pred }}\left(\theta^{\text {pred }}\right)$ coordinate is computed as the average of the predicted incidence angles over the group. The averages are weighted by uncertainty in both cases. For both methods, the resolution exhibits a minimum value at an incidence angle in the range of $25^{\circ}$ to $45^{\circ}$, dependent on the pixel pitch. For larger incidence angles (measured in $v$-direction), the resolutions worsens. This is a well-known effect in tracking detectors [83]. The intrinsic spatial resolution in both $u$-direction and $v$-direction for every combination of the incidence angles and comparing the COG PFA and the CSA approach is given in Table 8.9. The intrinsic spatial resolution provided by the CSA approach is at least as good as the COG PFA method, also for intermediate incidence angles where the linear charge sharing assumption of the COG PFA is most appropriate. The best intrinsic spatial resolution, with feasible uncertainty, in $u$-direction is $5.9(6) \mu \mathrm{m}$ obtained with the CSA at a predicted incidence angle of $\phi^{\text {pred }}=28.3(1)^{\circ}$. In $v$-direction and a pixel pitch of $55 \mu \mathrm{m}$, the best resolution is 7.0(4) $\mu \mathrm{m}$ at an incidence angle of $\theta^{\text {pred }}=32.8(1)^{\circ}$. The systematic uncertainty on the intrinsic spatial resolution increases significantly for large incidence angles due to the increased distance between the telescope arms necessary to accommodate the tilted DUT. This worsens the pointing resolution and its systematic uncertainty. 

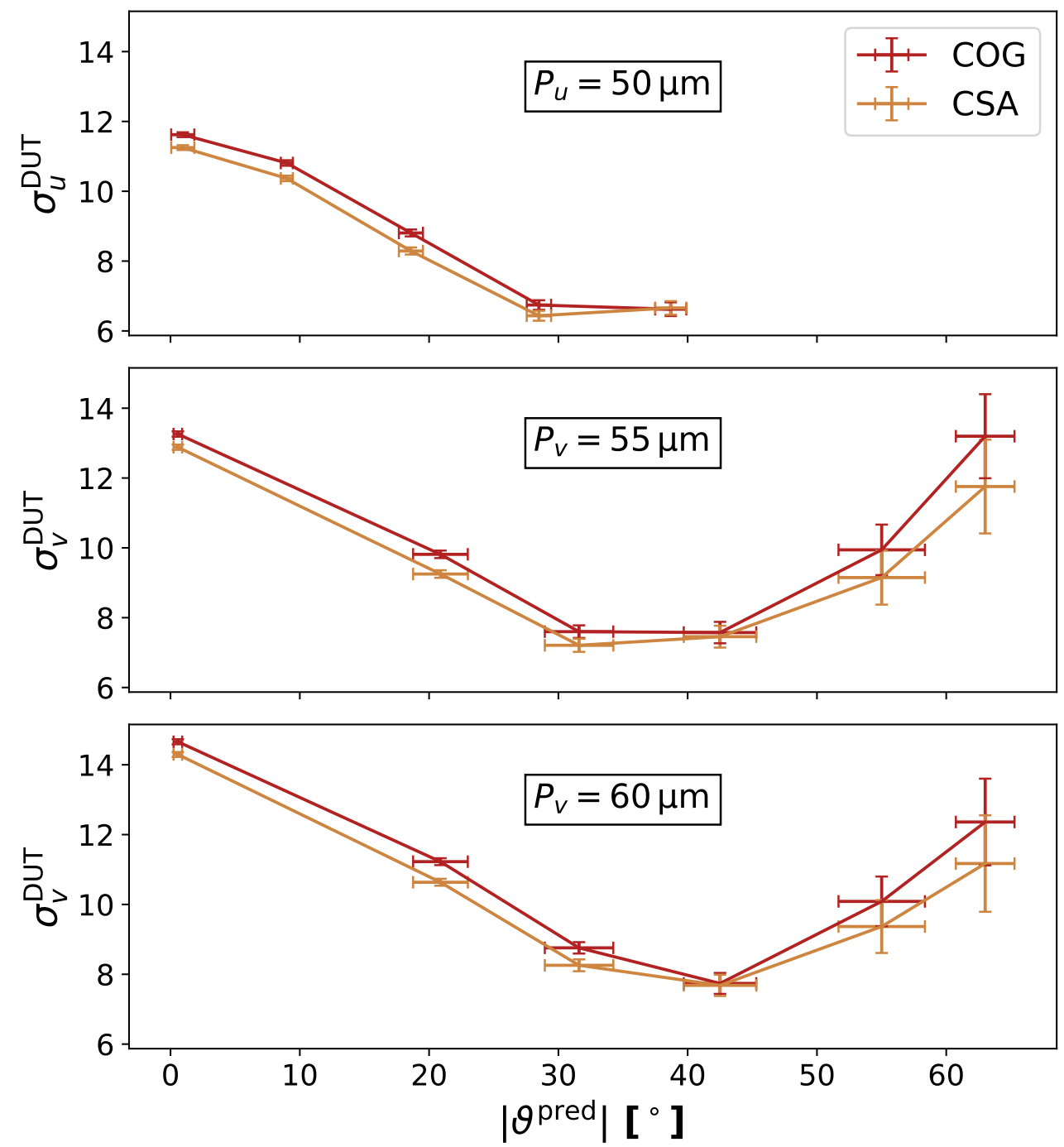

Figure 8.18.: Intrinsic spatial resolution (all cluster sizes) in $u$-direction and $v$-direction as a function of the respective incidence angle and per pixel pitch. Shown are the spatial resolutions obtained with the CPG PFA and the CSA approach. The uncertainties in spatial resolution are the combined (quadratically added) statistical and systematic uncertainty contributions. The generic horizontal axis label is to be understood as $\vartheta=\phi$ for the $u$-direction and $\vartheta=\theta$ for the $v$-direction. 


\begin{tabular}{cccccc}
\hline$\left|\phi^{\text {pred }}\right|\left[^{\circ}\right]$ & $\left|\theta^{\text {pred }}\right|\left[^{\circ}\right]$ & $n_{\mathcal{S}}$ & $n_{\mathcal{S} \rho}$ & coverage $[\%]$ & $n_{\mathcal{C}}\left[\times 10^{6}\right]$ \\
\hline $0.0(1)$ & $0.0(1)$ & 25 & 113 & 99.5 & 0.30 \\
$0.1(1)$ & $18.8(1)$ & 25 & 114 & 99.5 & 0.28 \\
$0.4(1)$ & $29.0(1)$ & 33 & 125 & 99.4 & 0.45 \\
$0.9(1)$ & $39.7(1)$ & 41 & 133 & 99.2 & 0.37 \\
$1.6(1)$ & $50.3(1)$ & 60 & 213 & 99.0 & 0.36 \\
$2.5(1)$ & $60.2(1)$ & 32 & 85 & 96.3 & 0.03 \\
$8.4(1)$ & $40.1(1)$ & 44 & 150 & 99.2 & 0.38 \\
$8.8(1)$ & $29.4(1)$ & 36 & 136 & 99.3 & 0.47 \\
$9.1(1)$ & $19.1(1)$ & 26 & 122 & 99.4 & 0.38 \\
$9.7(1)$ & $0.4(1)$ & 37 & 143 & 99.6 & 0.73 \\
$10.3(1)$ & $60.4(1)$ & 12 & 99 & 99.9 & 0.09 \\
$17.0(1)$ & $61.9(1)$ & 86 & 287 & 98.2 & 0.34 \\
$18.3(1)$ & $41.5(1)$ & 52 & 162 & 99.1 & 0.40 \\
$18.7(1)$ & $30.6(1)$ & 38 & 122 & 99.1 & 0.39 \\
$19.3(1)$ & $20.1(1)$ & 31 & 116 & 99.3 & 0.35 \\
$19.7(1)$ & $0.6(1)$ & 27 & 117 & 99.4 & 0.32 \\
$26.9(1)$ & $63.7(1)$ & 84 & 321 & 97.5 & 0.33 \\
$28.3(1)$ & $43.8(1)$ & 59 & 180 & 98.9 & 0.37 \\
$28.6(1)$ & $32.8(1)$ & 42 & 117 & 98.9 & 0.28 \\
$29.0(1)$ & $21.9(1)$ & 33 & 110 & 99.0 & 0.27 \\
$29.7(1)$ & $0.9(1)$ & 35 & 119 & 99.3 & 0.28 \\
$36.9(1)$ & $66.3(1)$ & 88 & 328 & 96.5 & 0.23 \\
$37.7(1)$ & $57.5(1)$ & 73 & 251 & 97.8 & 0.33 \\
$38.2(1)$ & $47.3(1)$ & 75 & 219 & 98.7 & 0.41 \\
$38.6(1)$ & $36.2(1)$ & 50 & 139 & 98.8 & 0.28 \\
$38.9(1)$ & $24.5(1)$ & 48 & 151 & 99.0 & 0.36 \\
$39.8(1)$ & $0.9(1)$ & 32 & 121 & 98.9 & 0.18 \\
$40.8(1)$ & $57.3(1)$ & 78 & 277 & 97.9 & 0.30 \\
\hline & & & & &
\end{tabular}

Table 8.8.: Composition of the CSA calibration per absolute value of the incidence angles. Given are the number of distinct cluster shapes $c_{\mathcal{S}}$ found in a calibration sample of $n_{\mathcal{C}}$ clusters. After finding the optimal $\rho$-binning for each shape, a total number of $n_{\rho} \mathcal{S} \rho$-S-bins is available in the lookup table. The coverage is the fraction of clusters of the training sample for which, after calibration, a matching $\rho$-S-bin is available. 


\begin{tabular}{rr|rr|rr}
\hline & & \multicolumn{2}{|c}{$\sigma_{u}^{\text {DUT }}[\mu \mathrm{m}]$} & \multicolumn{2}{c}{$\sigma_{v}^{\text {DUT }}[\mu \mathrm{mm}]$} \\
$\left|\phi^{\text {pred }}\right|\left[^{\circ}\right]$ & \multicolumn{1}{|c|}{$\theta^{\text {pred }} \mid\left[^{\circ}\right]$} & by COG PFA & by CSA & by COG PFA & by CSA \\
\hline $0.0(1)$ & $0.0(1)$ & $11.7 \pm 0.1 \pm 0.1$ & $11.4 \pm 0.1 \pm 0.1$ & $13.2 \pm 0.1 \pm 0.1$ & $12.9 \pm 0.1 \pm 0.1$ \\
$0.1(1)$ & $18.8(1)$ & $11.7 \pm 0.1 \pm 0.1$ & $11.4 \pm 0.1 \pm 0.1$ & $10.3 \pm 0.1 \pm 0.1$ & $9.8 \pm 0.1 \pm 0.1$ \\
$0.4(1)$ & $29.0(1)$ & $11.7 \pm 0.1 \pm 0.2$ & $11.3 \pm 0.1 \pm 0.2$ & $8.1 \pm 0.2 \pm 0.4$ & $7.6 \pm 0.2 \pm 0.4$ \\
$0.9(1)$ & $39.7(1)$ & $11.6 \pm 0.2 \pm 0.2$ & $11.1 \pm 0.1 \pm 0.2$ & $7.4 \pm 0.2 \pm 0.5$ & $7.2 \pm 0.2 \pm 0.5$ \\
$1.6(1)$ & $50.3(1)$ & $11.6 \pm 0.2 \pm 0.3$ & $11.0 \pm 0.2 \pm 0.3$ & $10.0 \pm 0.2 \pm 0.9$ & $9.4 \pm 0.2 \pm 0.9$ \\
$2.5(1)$ & $60.2(1)$ & $11.3 \pm 0.2 \pm 0.7$ & $10.6 \pm 0.2 \pm 0.7$ & $10.0 \pm 0.4 \pm 3.0$ & $8.5 \pm 0.4 \pm 3.5$ \\
$9.7(1)$ & $0.4(1)$ & $10.8 \pm 0.1 \pm 0.2$ & $10.4 \pm 0.1 \pm 0.2$ & $13.3 \pm 0.1 \pm 0.1$ & $12.9 \pm 0.1 \pm 0.1$ \\
$9.1(1)$ & $19.1(1)$ & $10.9 \pm 0.2 \pm 0.2$ & $10.5 \pm 0.1 \pm 0.2$ & $10.3 \pm 0.2 \pm 0.2$ & $9.7 \pm 0.1 \pm 0.2$ \\
$8.8(1)$ & $29.4(1)$ & $10.9 \pm 0.2 \pm 0.1$ & $10.4 \pm 0.2 \pm 0.1$ & $8.0 \pm 0.2 \pm 0.3$ & $7.5 \pm 0.2 \pm 0.3$ \\
$8.4(1)$ & $40.1(1)$ & $10.8 \pm 0.2 \pm 0.2$ & $10.2 \pm 0.2 \pm 0.2$ & $7.4 \pm 0.2 \pm 0.5$ & $7.2 \pm 0.2 \pm 0.6$ \\
$19.7(1)$ & $0.6(1)$ & $9.0 \pm 0.2 \pm 0.2$ & $8.5 \pm 0.2 \pm 0.2$ & $13.4 \pm 0.1 \pm 0.1$ & $13.0 \pm 0.1 \pm 0.1$ \\
$19.3(1)$ & $20.1(1)$ & $8.9 \pm 0.2 \pm 0.2$ & $8.4 \pm 0.2 \pm 0.2$ & $10.0 \pm 0.2 \pm 0.2$ & $9.5 \pm 0.2 \pm 0.2$ \\
$18.7(1)$ & $30.6(1)$ & $8.8 \pm 0.2 \pm 0.2$ & $8.2 \pm 0.2 \pm 0.2$ & $7.7 \pm 0.2 \pm 0.3$ & $7.4 \pm 0.2 \pm 0.3$ \\
$18.3(1)$ & $41.5(1)$ & $8.8 \pm 0.2 \pm 0.5$ & $8.1 \pm 0.2 \pm 0.5$ & $8.2 \pm 0.2 \pm 1.0$ & $8.2 \pm 0.2 \pm 1.0$ \\
$17.0(1)$ & $61.9(1)$ & $8.5 \pm 0.2 \pm 0.7$ & $7.2 \pm 0.2 \pm 0.8$ & $12.5 \pm 0.2 \pm 1.9$ & $11.5 \pm 0.2 \pm 2.1$ \\
$29.7(1)$ & $0.9(1)$ & $7.3 \pm 0.2 \pm 0.2$ & $7.0 \pm 0.2 \pm 0.3$ & $13.4 \pm 0.1 \pm 0.1$ & $13.0 \pm 0.1 \pm 0.1$ \\
$29.0(1)$ & $21.9(1)$ & $6.8 \pm 0.2 \pm 0.3$ & $6.5 \pm 0.2 \pm 0.3$ & $9.5 \pm 0.2 \pm 0.2$ & $8.9 \pm 0.2 \pm 0.2$ \\
$28.6(1)$ & $32.8(1)$ & $6.7 \pm 0.2 \pm 0.4$ & $6.3 \pm 0.2 \pm 0.4$ & $7.3 \pm 0.2 \pm 0.4$ & $7.0 \pm 0.2 \pm 0.4$ \\
$28.3(1)$ & $43.8(1)$ & $6.4 \pm 0.2 \pm 0.5$ & $5.9 \pm 0.2 \pm 0.6$ & $8.3 \pm 0.2 \pm 0.7$ & $8.4 \pm 0.2 \pm 0.7$ \\
$26.9(1)$ & $63.7(1)$ & $6.3 \pm 0.2 \pm 1.1$ & $5.4 \pm 0.3 \pm 1.3$ & $14.7 \pm 0.2 \pm 2.2$ & $13.4 \pm 0.2 \pm 2.3$ \\
$39.8(1)$ & $0.9(1)$ & $7.8 \pm 0.2 \pm 0.6$ & $7.7 \pm 0.2 \pm 0.6$ & $13.5 \pm 0.1 \pm 0.2$ & $13.0 \pm 0.1 \pm 0.2$ \\
$38.9(1)$ & $24.5(1)$ & $6.9 \pm 0.2 \pm 0.4$ & $7.0 \pm 0.2 \pm 0.4$ & $8.7 \pm 0.2 \pm 0.2$ & $7.9 \pm 0.2 \pm 0.3$ \\
$38.6(1)$ & $36.2(1)$ & $6.9 \pm 0.2 \pm 0.5$ & $7.1 \pm 0.2 \pm 0.5$ & $7.0 \pm 0.2 \pm 0.5$ & $7.0 \pm 0.2 \pm 0.5$ \\
$38.2(1)$ & $47.3(1)$ & $6.6 \pm 0.2 \pm 0.7$ & $6.7 \pm 0.2 \pm 0.7$ & $9.3 \pm 0.2 \pm 0.8$ & $9.2 \pm 0.2 \pm 0.8$ \\
$37.7(1)$ & $57.5(1)$ & $6.5 \pm 0.2 \pm 1.1$ & $6.4 \pm 0.2 \pm 1.1$ & $11.7 \pm 0.2 \pm 1.5$ & $10.8 \pm 0.2 \pm 1.6$ \\
$37.7(1)$ & $57.5(1)$ & $6.8 \pm 0.3 \pm 1.2$ & $6.4 \pm 0.2 \pm 1.1$ & $10.8 \pm 0.2 \pm 1.6$ & $10.8 \pm 0.2 \pm 1.6$ \\
$40.8(1)$ & $57.3(1)$ & $6.5 \pm 0.2 \pm 1.1$ & $6.6 \pm 0.3 \pm 1.2$ & $11.7 \pm 0.2 \pm 1.5$ & $9.8 \pm 0.2 \pm 1.8$ \\
$40.8(1)$ & $57.3(1)$ & $6.8 \pm 0.3 \pm 1.2$ & $6.6 \pm 0.3 \pm 1.2$ & $10.8 \pm 0.2 \pm 1.6$ & $9.8 \pm 0.2 \pm 1.8$ \\
$36.9(1)$ & $66.3(1)$ & $6.8 \pm 0.2 \pm 1.4$ & $6.2 \pm 0.2 \pm 1.5$ & $17.7 \pm 0.2 \pm 2.4$ & $15.7 \pm 0.2 \pm 2.6$ \\
\hline & & & & & \\
\hline
\end{tabular}

Table 8.9.: Intrinsic spatial resolution (over all cluster sizes) in $u$-direction and $v$ direction (for pitch $P_{v}=55 \mu \mathrm{m}$ ) using the COG PFA and the CSA approach as a function of the absolute value of the incidence angles. The uncertainties on the intrinsic resolution are given with statistical (first) contribution and systematic (second) contribution. The systematic uncertainties are fully correlated between the COG PFA and the CSA approach. 


\subsection{Hit efficiency studies}

The hit efficiency of a vertex detector is an important performance criterion. It measures the probability for the detector to produce a hit signal for a traversing charged particle. The ideal efficiency of $100 \%$ cannot be reached. The energy deposition is distributed according to a Landau distribution, with a small tail towards low energies, and the detector usually applies a signal threshold to reduce the occupancy by electrical noise. This excludes a certain fraction of particle passages from being registered. Also, the detector can be insensitive spatially - regions trapping charge before reaching any collecting electrode - , or temporally - a dead time of the charge collection or read-out electronics. The hit efficiency can be measured in beam tests using a beam telescope. For any reconstructed particle track that is extrapolated to the sensitive volume of the DUT, a DUT hit that can be matched to the extrapolated track is searched for. The fraction of tracks with a matched hit over all tracks is a measure for the DUT's hit efficiency. The following sections discuss the procedure and the obtained results. First, the track selection and the computation of the hit efficiency is defined, along with an estimation of the statistical and systematic uncertainty of the computation. The measured hit efficiencies of all DUTs are presented. The dependence of the hit efficiency on the zero-suppression threshold is investigated.

\subsubsection{Track selection and hit efficiency calculation}

The reconstruction of particle tracks using the beam telescope information is explained in Section 8.3.3. The extrapolation of these tracks to the DUT plane and matching of DUT hits is defined in Section 8.3.5. Only particle tracks that occurred within a common integration time window of all involved detectors can be considered for a sensible hit efficiency estimation. Requiring reconstructed tracks to have an associated hit on the FE-I4 detector plane with its fast $25 \mathrm{~ns}$ integration time assures that these tracks occurred within the overlap of the integration time of the DUT and the MIMOSA26 detectors of the beam telescope. In addition, tracks with an extrapolated DUT intersection coordinate that lies within the geometrical extent of a DEPFET pixel that is considered non-functional (cf. 8.3.1), or any adjacent neighbour thereof ${ }^{10}$, are rejected.

Given a set of $n$ tracks, fulfilling these criteria, the hit efficiency $\varepsilon$ is calculated as the ratio of the number $n_{\text {matched }}$ of tracks which have a matched hit on the DUT and the total number $n_{\text {total }}$ of tracks (matched and non-matched)

$$
\varepsilon=\frac{n_{\text {matched }}}{n_{\text {total }}} .
$$

As discussed in Section 8.3.5, clusters are matched to a track if the distance $d_{\text {match }}$ between the estimated cluster impinging coordinates and the extrapolated track intersection coordinates on the DUT plane is $d_{\text {match }} \leq 200 \mu \mathrm{m}$ both in $u$ - and $v$-direction

${ }^{10}$ Edge effects between functional and non-functional pixels are excluded by not considering pixels surrounding masked pixels. 


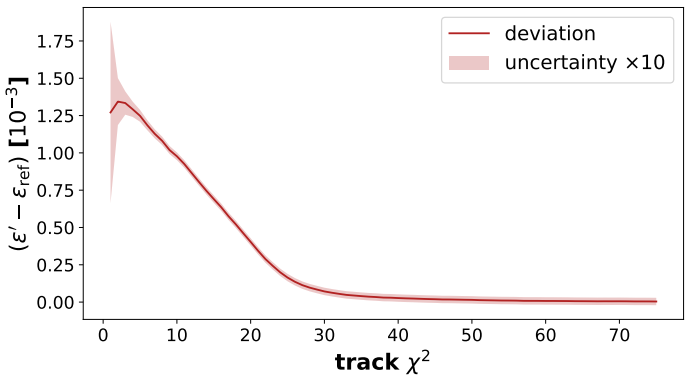

(a) track $\chi^{2}$ selection

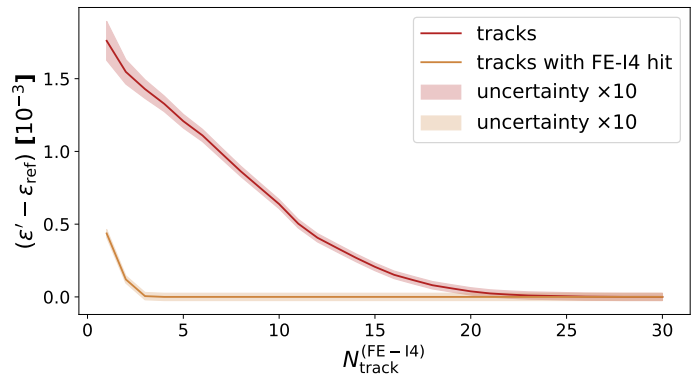

(b) track multiplicity selection

Figure 8.19.: Deviation of the selected hit efficiency $\varepsilon^{\prime}$ from the reference hit efficiency $\varepsilon_{\text {ref }}$ as a function of the threshold on the track $\chi^{2}$ value (a) and the (reference) track multiplicity (b). The statistical uncertainties are scaled by a factor of 10 for visibility.

independently. The statistical uncertainty on the hit efficiency, $\sigma_{\varepsilon}^{\text {stat }}$, is given by the variance of a binomial distribution

$$
\sigma_{\varepsilon}^{\text {stat }}=\sqrt{\frac{\varepsilon(1-\varepsilon)}{n_{\text {total }}}} .
$$

Assuming a hit efficiency of $\varepsilon=99 \%$, a number of $n_{\text {total }}=2500$ tracks is required to reach a statistical uncertainty of $\sigma_{\varepsilon}=0.2 \%$.

Ideally, the hit efficiency should be independent of any further track selection if the track reconstruction is robust. The systematic uncertainty on the hit efficiency is estimated based on the influence of track and event properties that are associated with potentially misreconstructed tracks. The hit efficiency is determined as a function of the track selection regarding the track $\chi^{2}$ value, and the track multiplicity $N_{\text {track }}$ and reference track multiplicity $N_{\text {track }}^{\mathrm{FE}-\mathrm{I} 4}$ (number of tracks with an associated hit on the FE-I4 detector plane) in the respective event. Misreconstructed - non-real - tracks cannot be associated to a DUT cluster and therfore impose an artificial inefficiency on the DUT. A large track $\chi^{2}$ value can be caused by the addition of random noise hits from detector planes in the track reconstruction. For a large number of reconstructed tracks in an event, the probability for ambiguities in the hit-to-track matching is increased. Incorrectly associated hits also yield misreconstructed tracks. The computed hit efficiency without any thresholds on the said variables is used as a reference value $\varepsilon_{\text {ref }}$. Figure 8.19 shows the deviation of the selected hit efficiency $\varepsilon^{\prime}$, with a more strict track selection applied in the given variable, from the reference hit efficiency . Significant deviations are observed for the selections $N_{\text {track }} \leq 5, N_{\text {track }}^{\mathrm{FE}-\mathrm{I} 4} \leq 1$ and $\chi^{2} \leq 10$ (the track reconstruction uses a threshold of $\chi^{2} \leq 100$ ), respectively. The overall systematic uncertainty is determined as the overall deviation of the selected hit efficiency with all above selections applied with respect to the reference value. This procedure, very consistently for all DUTs, yields a systematic uncertainty of $\sigma_{\varepsilon}^{\text {syst }}=0.15 \%$. Overall, the observed 


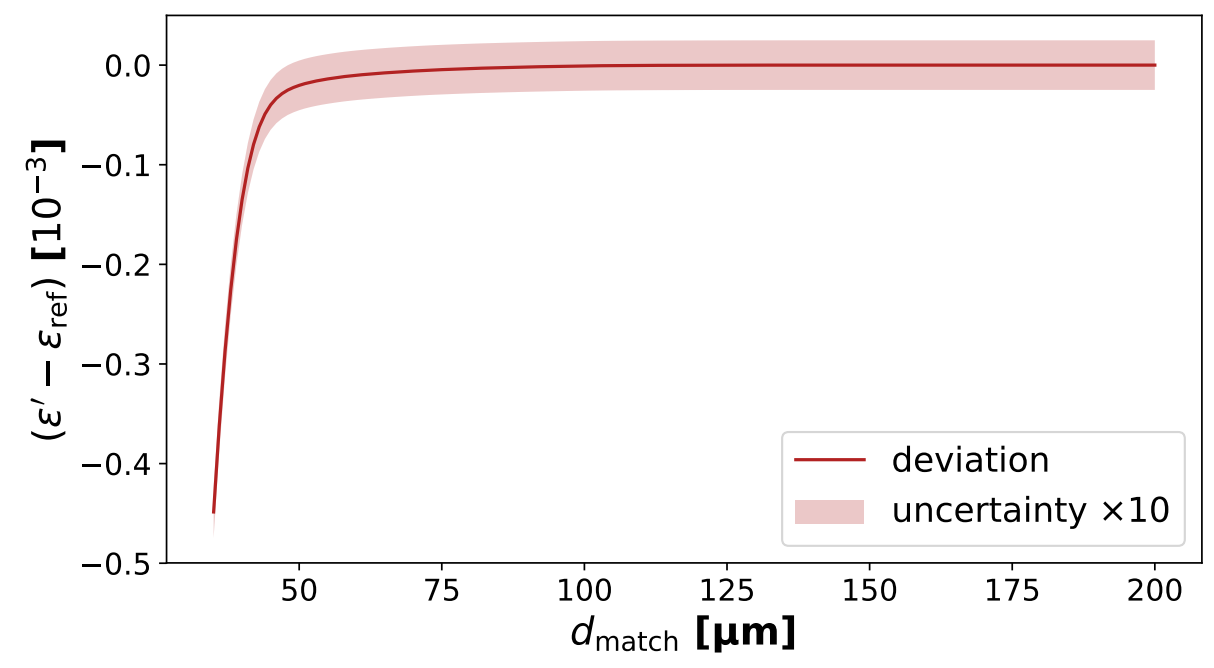

Figure 8.20.: Deviation of the selected hit efficiency $\varepsilon^{\prime}$ from the reference hit efficiency $\varepsilon_{\text {ref }}$ as a function of the matching distance $d_{\text {match. }}$. The statistical uncertainty is scaled by a factor of 10 for visibility.

dependencies are weak, confirming that the track reconstruction is reliable.

The systematic influence of the hit to track matching distance threshold was found to be negligible for values $d_{\text {match }} \geq 60 \mu \mathrm{m}$ as illustrated in Figure 8.20. In this regime, the distance criterion becomes effectively smaller than the geometrical extent of the pixels. The dependence is flat around the selected threshold of $200 \mu \mathrm{m}$, indicating that there is no significant influence on the hit efficiency by matching of uncorrelated (noise) hits. The overall dependence, with a significant deviation (larger than the $0.15 \%$ systematic uncertainty) starting only at thresholds below $35 \mu \mathrm{m}$, demonstrates that the alignment of the DUT with respect to the beam telescope is reliable within this order of magnitude.

Table 8.10 summarises the hit efficiencies of the tested DUTs and the respective fractions of masked pixels. The overall average hit efficiency over the measured DUTs is 99.6\%. All DUTs are compatible with this value within the statistical and systematic uncertainty. The fraction of masked pixels has to be subtracted from the given hit efficiency to obtain an effective hit efficiency valid for the full sensor. It should be noted, however, that the investigated DUTs are lower grade modules with particular defects. Also, not the full extent of the sensitive area has been illuminated for all DUTs.

\subsubsection{Spatially resolved hit efficiency}

The hit efficiency can be determined as a function of the extrapolated track intersection coordinate on the DUT plane. The density of reconstructed tracks over the sensor area is not uniform due to a non-uniform particle density in the beam spot, variations in the exposure time among the illumination windows and the like. For the overall hit efficiency numbers given in Table 8.10, the hit efficiency of areas of the sensor enter weighted by the 


\begin{tabular}{lcc}
\hline DUT & $\varepsilon \pm$ (stat.) \pm (syst.) $[\%]$ & masked [\%] \\
\hline W40_IF & $99.55 \pm 0.001 \pm 0.15$ & 1.87 \\
W11_OF2 & $99.64 \pm 0.001 \pm 0.15$ & 5.35 \\
W05_OB1 & $99.63 \pm 0.002 \pm 0.15$ & 25.69 \\
W05_OB1 $^{\gamma}$ & $99.52 \pm 0.002 \pm 0.15$ & 31.82 \\
W37_OF1 & $99.49 \pm 0.001 \pm 0.15$ & 1.64 \\
\hline
\end{tabular}

Table 8.10.: Computed hit efficiencies $\varepsilon$ per DUT. Given is the overall hit efficiency with the statistical and systematic uncertainty. The last column gives the fraction of masked (hot or dead) pixels with respect to the $250 \times 768$ DEPFET pixels on each DUT.

\begin{tabular}{lccc}
\hline DUT & $\langle\varepsilon\rangle[\%]$ & $P_{5}(\varepsilon)[\%]$ & $P_{95}(\varepsilon)[\%]$ \\
\hline W40_IF & $99.5 \pm 0.6$ & 98.8 & 99.8 \\
W11_OF2 & $99.7 \pm 0.2$ & 99.3 & 99.8 \\
W05_OB1 $99.6 \pm 0.2$ & 99.2 & 99.8 \\
W05_OB1 $^{\gamma}$ & $99.5 \pm 1.2$ & 98.5 & 99.8 \\
W37_OF1 & $99.5 \pm 0.8$ & 98.7 & 99.7 \\
\hline
\end{tabular}

Table 8.11.: Average hit efficiency $\langle\varepsilon\rangle$ over all super-pixels per DUT. The uncertainty is the standard deviation over the super-pixels. The last two columns give the 5th and 95th percentile of the hit efficiency over the super-pixels, respectively.

local track density. This is intrinsic weighting is mitigated by computing the spatially resolved hit efficiency and subsequently averaging over the spatial bins. The binning of the sensitive area is chosen such that each bin collects a sufficient number of tracks, allowing for determining the efficiency at a low statistical uncertainty, while ensuring a uniform distribution of tracks over the bin. With a binning accumulating $u \times v=10 \times 8$ pixels into one bin, a super-pixel, the average number of reconstructed tracks per bin is about $10^{4}$. $96 \%$ of the super-pixel bins collect more than 4000 tracks. The remaining $4 \%$ of pixels collect on average 2600 tracks.

Figure 8.21 shows the hit efficiency per super-pixel for DUT W40_IF and a distribution of the hit efficiency over all super-pixel bins. An average hit efficiency of $\langle\varepsilon\rangle=99.5 \%$ is measured with a standard deviation of $0.6 \%$. The median is $99.7 \%$. The hit efficiency is mostly uniform over the sensor. Single super-pixel bins exhibit a significantly reduced efficiency. The pixel masking could be improved to identify non-functional pixels more precisely. The hit efficiency maps for the other DUTs are given in Appendix A. Table 8.11 lists the hit efficiency averaged over the super-pixel bins for all DUTs. Figure 8.22 expresses the distribution of hit efficiencies over the super-pixel bins as a boxplot. The spatially resolved hit efficiencies are in general in good agreement among the DUTs. The overall average yields a hit efficiency of $99.6 \%$. Single super-pixel bins exhibit a 

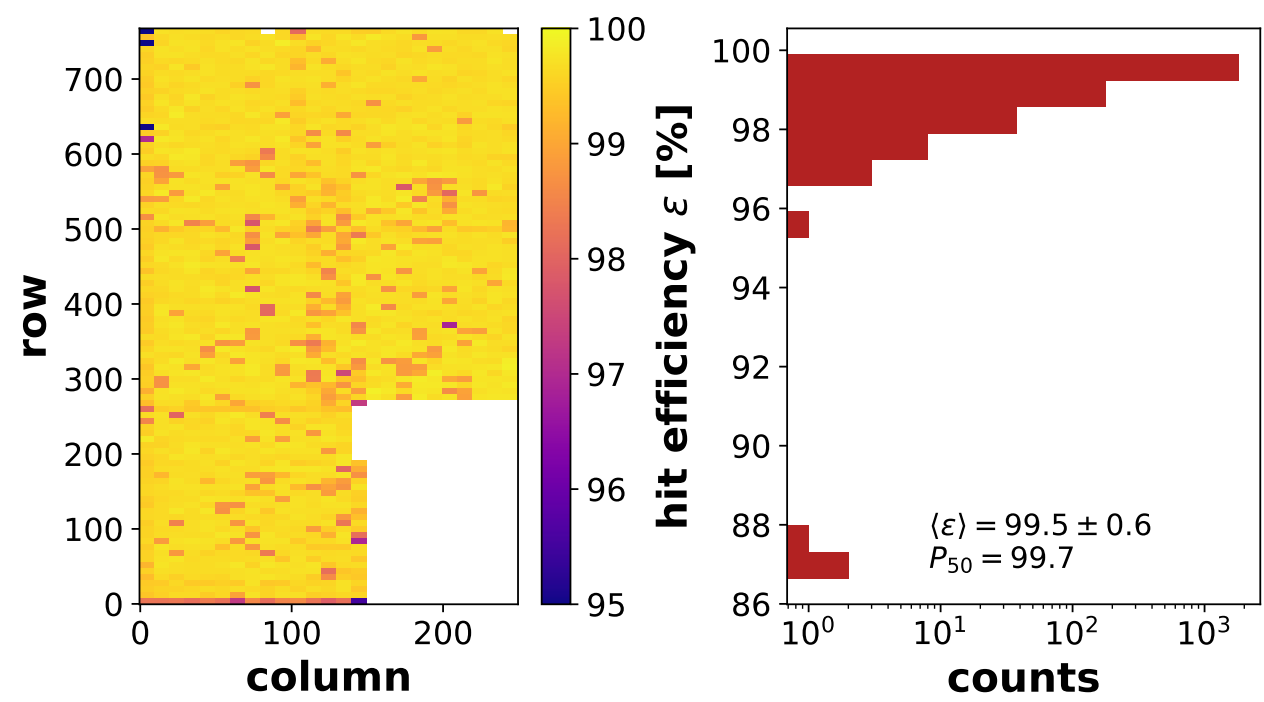

Figure 8.21.: Hit efficiency $\varepsilon$ as a function of the super-pixel coordinate and distribution over all super-pixels for DUT W40_IF.

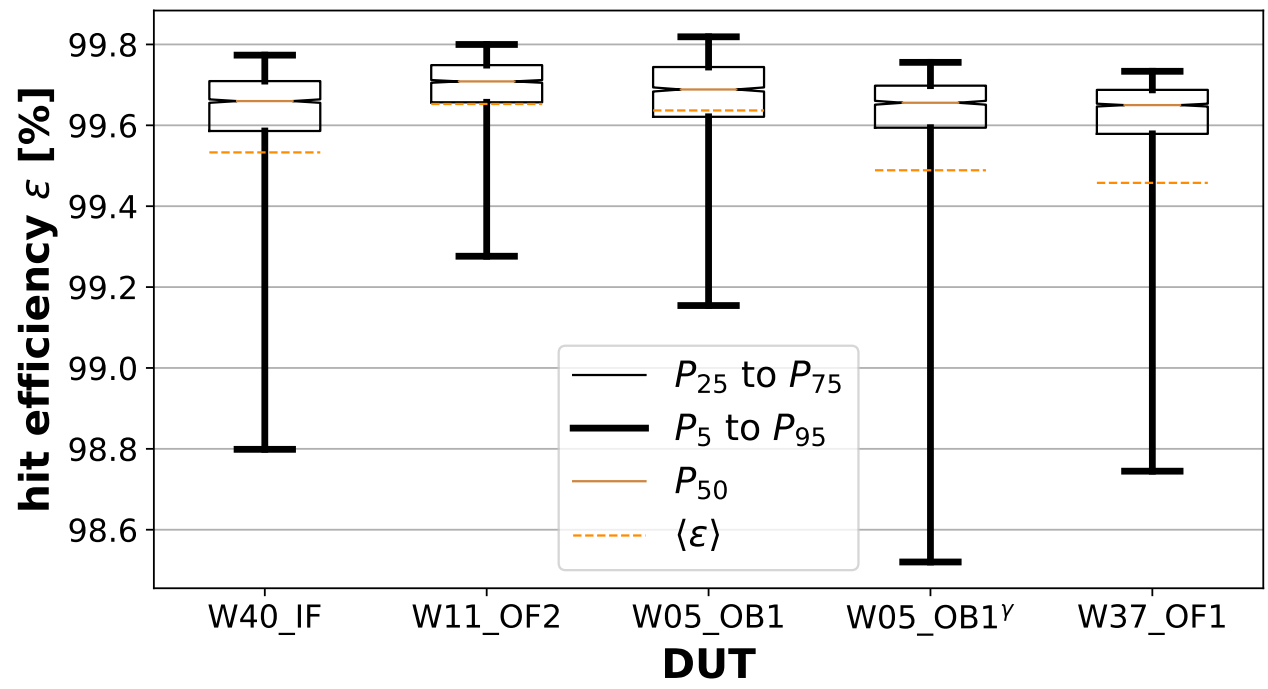

Figure 8.22.: Distribution of hit efficiencies over the super-pixels per DUT. Shown are the respective percentile ranges, the median $\left(P_{50}\right)$ and the average. 


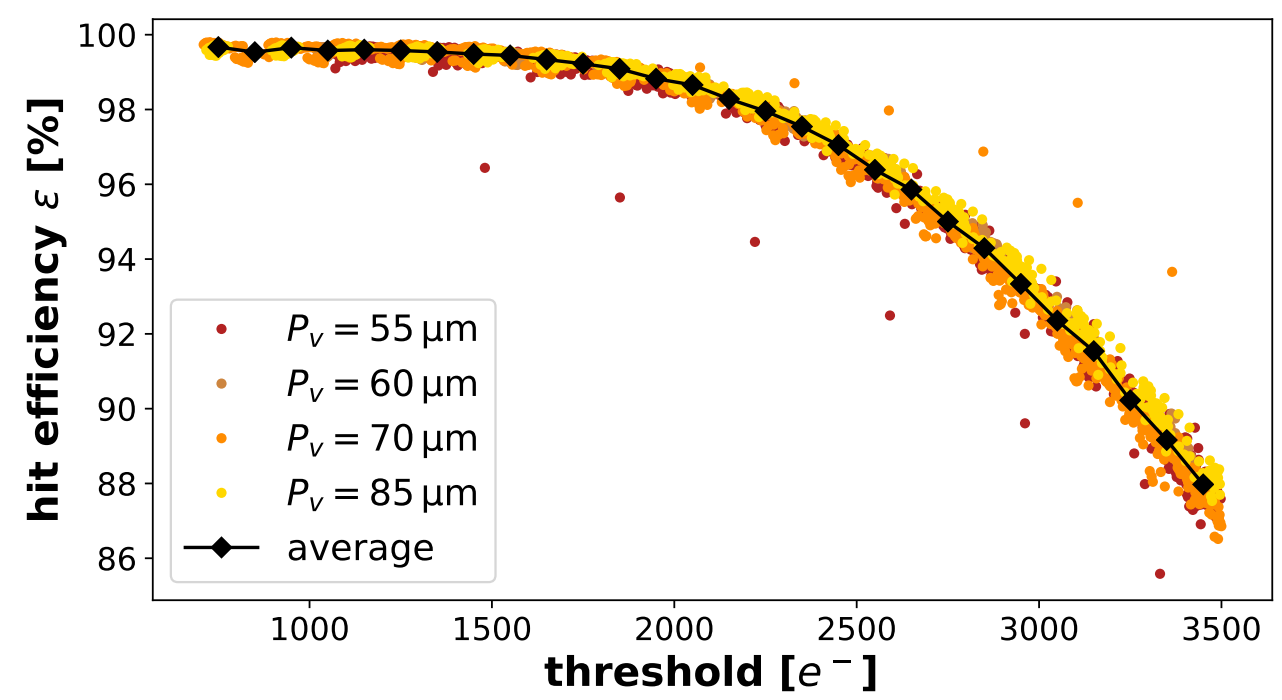

Figure 8.23.: Hit efficiency per four-row as a function of the applied signal threshold in number of electrons for all four distinct pixel pitches. The average is computed over all data points in consecutive threshold bins of 100 electrons.

reduced hit efficiency and result in a significant variation of the $P_{5}$ percentiles among the DUTs. The origin is most likely an effect of the normalised occupancy thresholds employed in the pixel masking. As seen in Figure 8.7, a small variation of the dead pixel threshold has a large impact on the selection of pixels to be masked. It is, however, more relevant to note that the other percentiles are in good agreement and that all DUTs can be operated with an average hit efficiency consistent with $99.6 \%$.

\subsubsection{Influence of the zero-suppression threshold}

The suppression of fake hits due to electronic noise is realised with an online zerosuppression threshold in the DHP ASICs. However, applying a signal threshold also means cutting the low-energy tail of the Landau-shaped charge deposition distribution, as motivated earlier. The influence of this threshold effect is studied by applying and varying an additional offline signal threshold (in units of ADU) and comparing the resulting hit efficiencies. Technically, the selection of matched tracks is extended by requiring the seed signal of a matched cluster to exceed the given offline threshold. Since mostly one-pixel clusters are observed at perpendicular incidence (cf. Fig. 8.9), this procedure is sufficiently accurate and a full re-clustering is not necessary. The offline threshold is translated to a more physical quantity, number of electrons, by employing the calibration constants. Since the calibrations are on a four-row basis, however, the hit efficiency as a function of the threshold in number of electrons is given per four-row of each DUT. Additionally, the dependence is investigated for each pixel pitch design individually. Figure 8.23 shows the obtained dependencies. The hit efficiency degrades 


\section{Measuring Pixel Detector Modules in Beam Tests}

for increasing thresholds, as expected. Based on the averaged dependence, a hit efficiency above $98 \%$ can be maintained for thresholds up to 2100 electrons. The few outliers are from a single four-row with a significant variation in hit efficiency on both devices. The origin is most likely the same pixel masking threshold effect that causes the variation in the $P_{5}$ percentiles in Figure 8.22, as discussed before.

\subsection{Sub-pixel resolved measurements}

The beam telescope provides an excellent pointing resolution of below $4 \mu \mathrm{m}$ at perpendicular incidence and for a beam energy of $3 \mathrm{GeV}$ and an appropriate telescope geometry (cf. Tab. 8.5). Detailed studies of the charge collection within the DEPFET pixels are thereby possible. Several properties have been probed at sub-pixel level. This comprises studies of the charge sharing regions by considering the probabilities of different cluster sizes and studies of the charge collection efficiency by considering cluster charge distributions and the hit efficiencies.

A four-fold pixel design (cf. Fig. 6.4) is used for the DEPFET pixel detector modules and the $2 \times 2$ neighbouring pixels share common structures. Hence, not single pixels, but the respective $2 \times 2$ grid of pixels should be considered the smallest unit of interest when measuring charge collection properties within the DEPFET cells. Every block of $2 \times 2$ pixels is subdivided into bins of size $5 \mu \mathrm{m} \times 5 \mu \mathrm{m}$. For each reconstructed track, the extrapolated track intersection coordinate with the DUT plane is used to select the respective sub-pixel bin. The cluster size and cluster charge (of an associated DUT cluster) are then attributed to that sub-pixel bin. The information whether the respective track has an associated DUT cluster is used to determine the hit efficiency at sub-pixel level. The DUT cluster to track matching is done as before (cf. Sec. 8.6.1). The track density in the recorded data is insufficient to compute sub-pixel spatially resolved properties over the full DEPFET pixel matrix. Instead, a generic unit cell is defined and all equal sized blocks of pixels are overlaid in this unit cell. For also being sensitive to effects in the vicinity of the four-fold design, a $4 \times 4$ pixel grid is selected as the generic unit cell. For each DUT and $P_{v}$ pixel pitch region, an individual unit cell is considered.

The calibrated cluster energy MPV, $Q_{\mathrm{MPV}}$, is determined by fitting of a LanGau function to the cluster energy distribution per sub-pixel bin of the unit cell. Figure 8.25 shows the value of $Q_{\mathrm{MPV}}$ as a function of the sub-pixel bin for the four $P_{v}$ pixel pitches. The projections in $u$-direction and $v$-directions are given with the average and the standard deviation of $Q_{\mathrm{MPV}}$. The cluster energy MPV is mostly uniform over the unit cells, indicating that there is no systematic charge collection inefficiency within or in between DEPFET cells. A slightly reduced $Q_{\mathrm{MPV}}$ at pixel borders compared to the pixel centres is observed. The magnitude of the variations, however, are in the order of $0.6 \mathrm{keV}$ (overall standard deviation) which is within the combined systematic uncertainty of the LanGau MPV fit $(0.2 \mathrm{keV})$ and of the calibration factors $(0.4 \mathrm{keV})$. It can be concluded, that there is no region within the DEPFET cells that exhibit a significant charge loss.

Charge sharing among neighbouring pixels is essential for improving the resolution of 
the estimation of impinging coordinates from clusters, as discussed in Section 8.5. The charge sharing region - the geometrical extent a particle has to traverse to produce a charge cloud that is likely shared among pixels - is evaluated by considering the cluster size distribution per sub-pixel bin. For each bin, the probability $p(\Pi)$ for a particle track extrapolated to that bin to cause a DUT pixel cluster of total size $\Pi$ is computed as the fraction of clusters of size $\Pi$ over all clusters. Figure 8.24a shows the cluster size probability for sizes $\Pi=1, \Pi=2$ and $\Pi \geq 3$ at perpendicular beam incidence. A particle traversing the central region of a pixel causes essentially only single pixel clusters. Crossing the edge between two pixels, a particle is likely $(p(\Pi=2) \approx 70 \%)$ to produce a two-pixel cluster. Larger multi-pixel clusters $(\Pi \geq 3)$ are caused by particles traversing a small area around the corners of pixels $(p(\Pi \geq 3) \approx 30 \%)$. The charge sharing region has an extent of about two sub-pixel bins from the geometrical edge of the pixel, corresponding to a width of about $10 \mu \mathrm{m}$ at perpendicular incidence. The subpixel cluster size probability for inclined beam incidence at $\phi=30^{\circ}$ and $\theta=30^{\circ}$ is shown in Figure 8.24b. The single pixel region is drastically reduced. Multi-pixel clusters are dominating and the charge sharing region has an extent of roughly four sub-pixel bins corresponding to a width of about $20 \mu \mathrm{m}$. These findings support the observation that at inclined particle incidence of $\phi=30^{\circ}$ and $\theta=30^{\circ}$ the intrinsic resolution is improved by exploiting the increased charge sharing (cf. Sec. 8.5.3).

The hit efficiency was determined at sub-pixel resolution and the results for the unit cells of the four pixel pitches are shown in Figure 8.26. The hit efficiency is largely uniform over the geometrical extent of the DEPFET cells. This is in consistence with the uniformity of the cluster charge MPV (cf. Fig. 8.25). However, some variations are observed. The leftmost column of the $P_{v}=55 \mu \mathrm{m}$ pitch and the $P_{v}=70 \mu \mathrm{m}$ pitch, and the bottommost row of the $P_{v}=85 \mu \mathrm{m}$ pitch $4 \times 4$ pixel grid exhibit a slightly reduced hit efficiency of about $0.1 \%$ compared to the other pixels of the grid. The reduction is a general offset and thus not an effect of the DEPFET cell structure at sub-pixel level. The origin are individual columns (rows) of the pixel matrix with reduced hit efficiency (cf. App. A). A slight reduction in hit efficiency in pixel centres compared to pixel borders is observed, most prominent in the $v$-projection for pitch $P_{v}=70 \mu \mathrm{m}$. Overall, the observed variations are not exceeding $0.1 \%$ and are thus within the systematic uncertainty. Table 8.12 lists the hit efficiency averaged over the unit cells. The uncertainty is given by the standard deviation over all sub-pixel bins. The average hit efficiency for all unit cells is compatible to the value of $99.6 \%$ which was obtained as the average over all super-pixels for all measured DUTs. No significant degradation of the hit efficiency within the extent of single DEPFET cells is observed. 

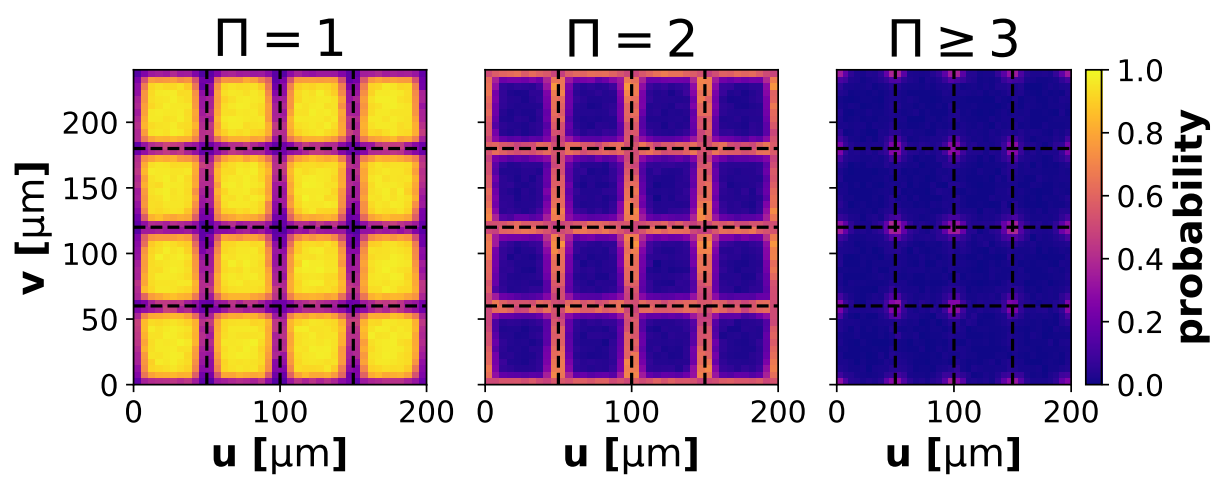

(a) perpendicular incidence
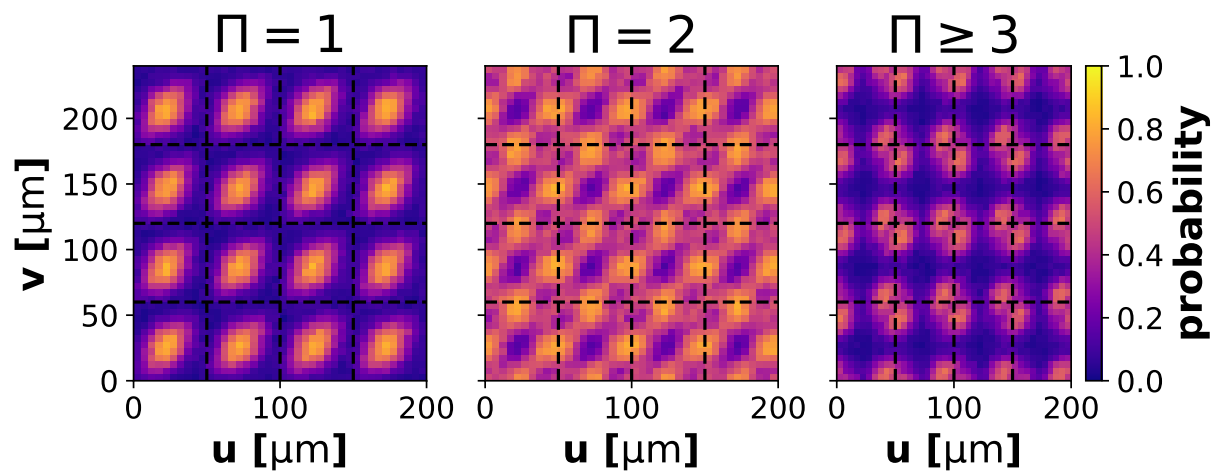

(b) inclined incidence at $\phi=30^{\circ}$ and $\theta=30^{\circ}$

Figure 8.24.: Sub-pixel resolved cluster size probability within a $4 \times 4$ DEPFET pixels unit cell for pixel pitch $50 \mu \mathrm{m} \times 60 \mu \mathrm{m}$ for perpendicular (a) and inclined (b) particle incidence. The dotted lines indicate the geometrical extent of the pixels.

\begin{tabular}{cccc}
\hline$P_{u} \times P_{v}[\mu \mathrm{m} \times \mu \mathrm{m}]$ & $\langle\varepsilon\rangle[\%]$ & $P_{1}(\varepsilon)[\%]$ & $P_{99}(\varepsilon)[\%]$ \\
\hline $50 \times 55$ & $99.6(1)$ & 99.3 & 99.8 \\
$50 \times 60$ & $99.6(1)$ & 99.2 & 99.7 \\
$50 \times 70$ & $99.6(1)$ & 99.3 & 99.8 \\
$50 \times 85$ & $99.7(1)$ & 99.4 & 99.9 \\
\hline
\end{tabular}

Table 8.12.: Averaged hit efficiency over the unit cells of the four pixel pitch designs. The average is weighted by the statistical uncertainty per sub-pixel bin, which is in the order of $2 \times 10^{-5}$. The indicated uncertainty on the average is given by the standard deviation of the hit efficiency over the sub-pixel bins. The last two columns give the first and 99th percentile of the hit efficiency over the sub-pixel bins, respectively. 


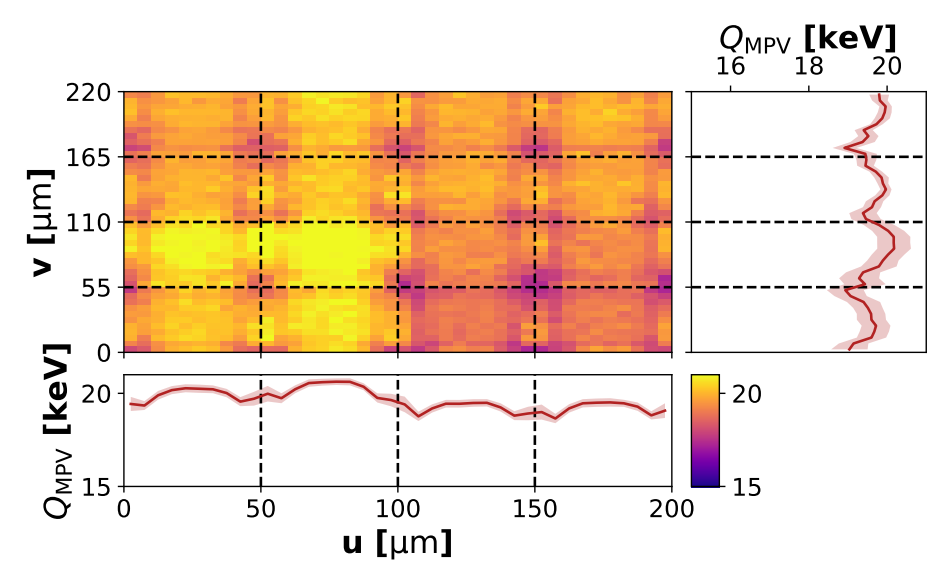

(a) unit cell for pixel pitch $50 \mu \mathrm{m} \times 55 \mu \mathrm{m}$

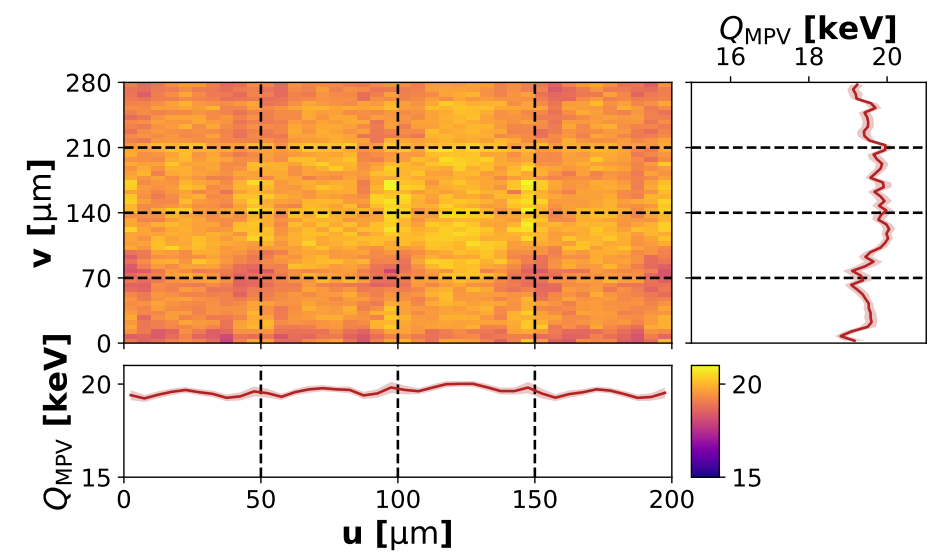

(c) unit cell for pixel pitch $50 \mu \mathrm{m} \times 70 \mu \mathrm{m}$

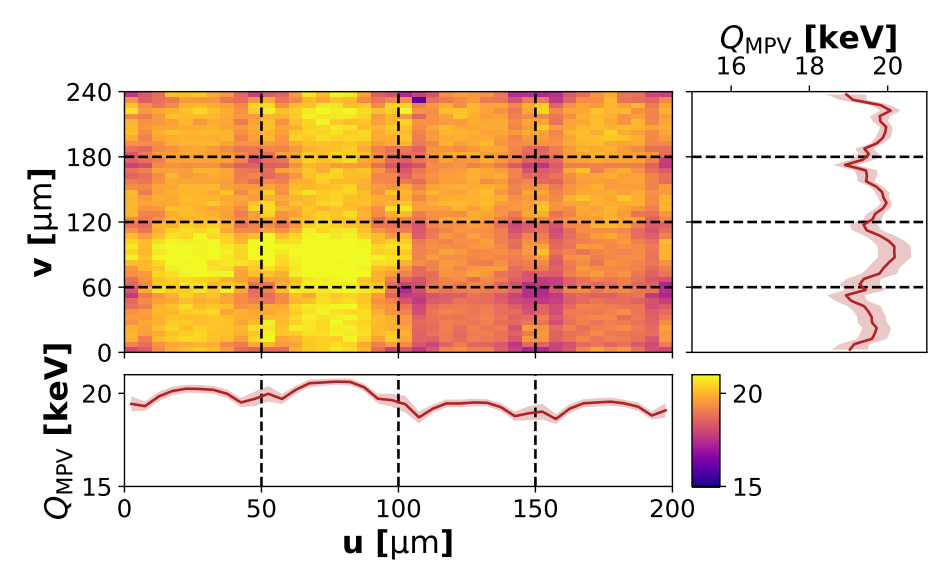

(b) unit cell for pixel pitch $50 \mu \mathrm{m} \times 60 \mu \mathrm{m}$

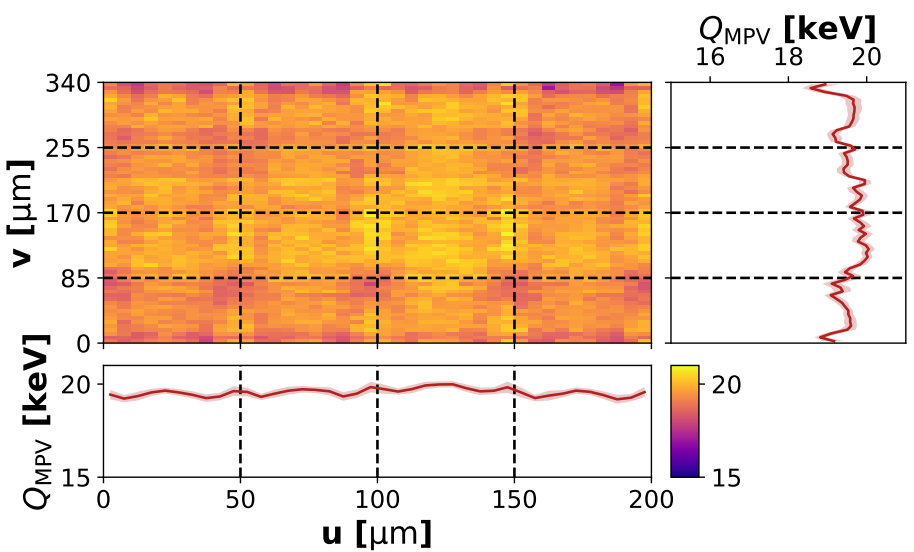

(d) unit cell for pixel pitch $50 \mu \mathrm{m} \times 85 \mu \mathrm{m}$

Figure 8.25.: Sub-pixel resolved cluster energy MPV, $Q_{\mathrm{MPV}}$, within a $4 \times 4$ DEPFET pixels unit cell for the four different $P_{v}$ pixel pitch designs. The dotted lines indicate the geometrical extent of the pixels. The projections in $u$-direction and $v$-direction, respectively, show the average (solid line) and the standard deviation (light band) over the projected spatial bins. 


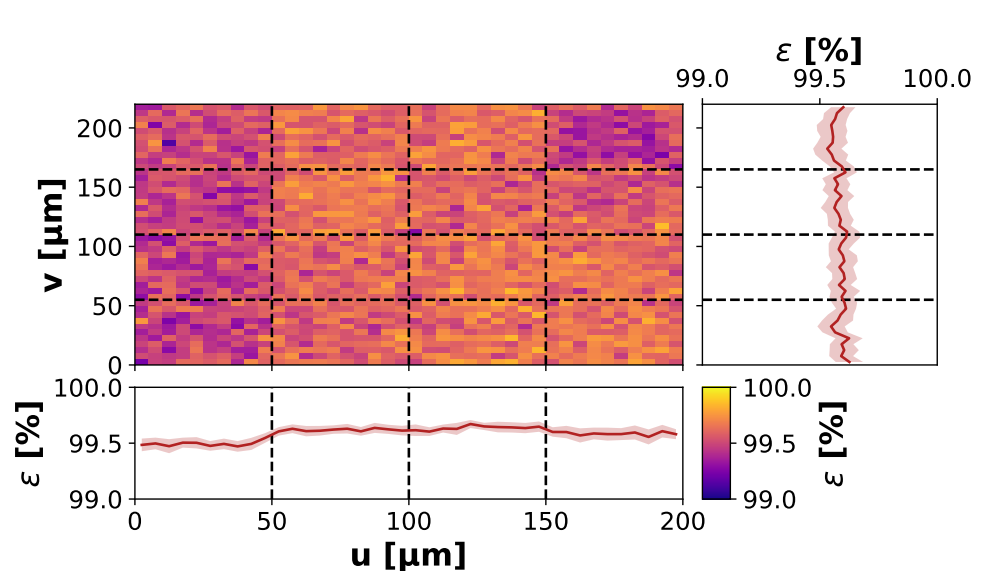

(a) unit cell for pixel pitch $50 \mu \mathrm{m} \times 55 \mu \mathrm{m}$

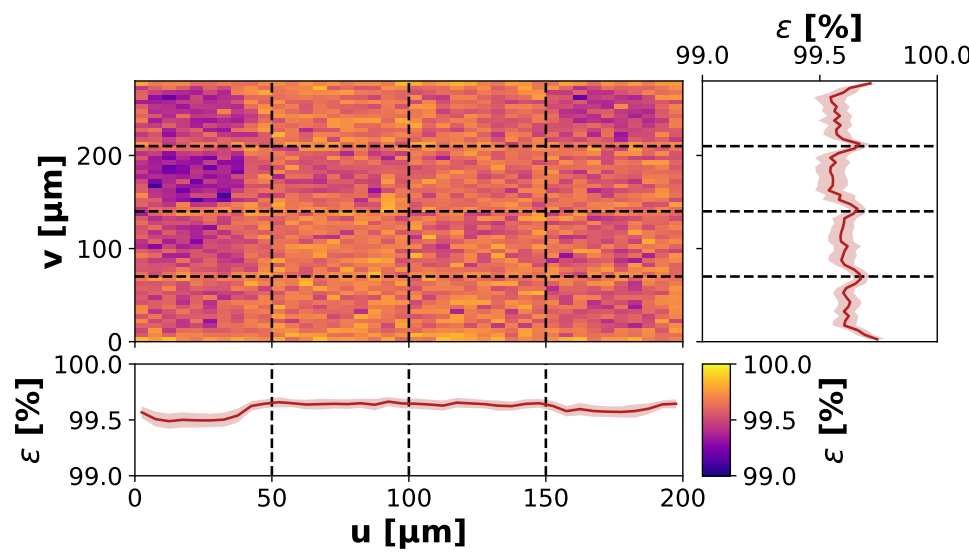

(c) unit cell for pixel pitch $50 \mu \mathrm{m} \times 70 \mu \mathrm{m}$

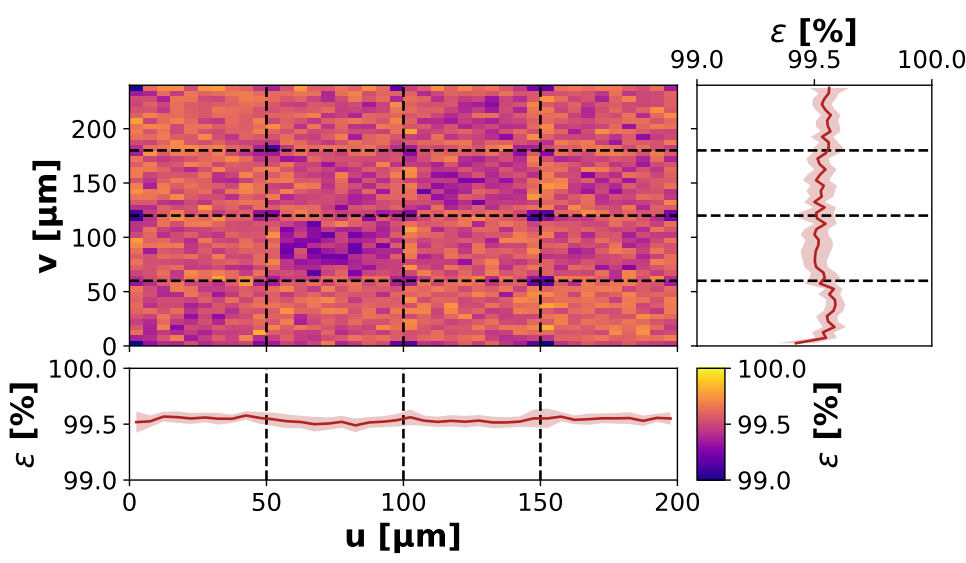

(b) unit cell for pixel pitch $50 \mu \mathrm{m} \times 60 \mu \mathrm{m}$

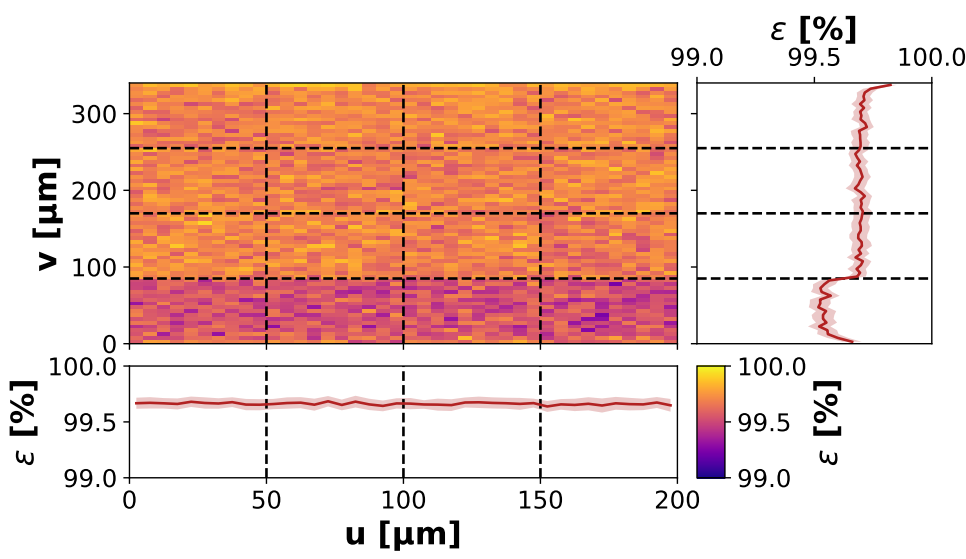

(d) unit cell for pixel pitch $50 \mu \mathrm{m} \times 85 \mu \mathrm{m}$

Figure 8.26.: Sub-pixel resolved hit efficiency $\varepsilon$ within a $4 \times 4$ DEPFET pixels unit cell per pixel pitch design. The dotted lines indicate the geometrical extent of the pixels. The projections in $u$-direction and $v$-direction, respectively, show the average (solid line) and the standard deviation (light band) over the projected spatial bins. 


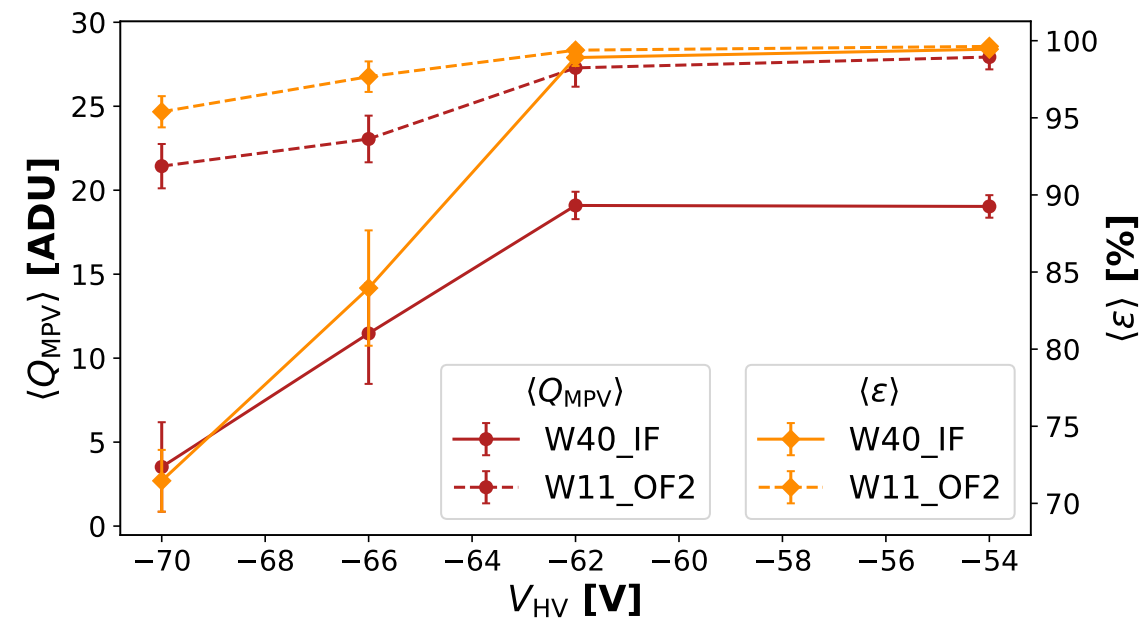

Figure 8.27.: Cluster charge MPV $Q_{\mathrm{MPV}}$ and hit efficiency $\varepsilon$, averaged over all superpixels, as a function of depletion voltage $V_{\mathrm{HV}}$ for the non-optimal biasing scenario $\left(V_{\text {drift }}=-4 \mathrm{~V}, V_{\text {clear }- \text { off }}=5 \mathrm{~V}\right)$ for DUTs W40_IF and W11_OF2.

\subsection{Influence of the DEPFET biasing}

The results presented so far were measured with the DUTs at optimised DEPFET biasing potentials (cf. Tab. 8.2). As discussed in Section 7.3, a non-optimal biasing scenario has a significant impact on the charge collection efficiency - approximated by measuring the degradation in cluster charge MPV - and results in non-uniform (ring-like) variations across the sensor. A particularly unfavourable biasing scenario of $V_{\text {drift }}=-4 \mathrm{~V}$ and $V_{\text {clear-off }}=5 \mathrm{~V}$ at different depletion voltages $V_{\mathrm{HV}}$ was applied to the DUTs W40_IF and W11_OF2 to study the impact of the DEPFET biasing on the hit efficiency. The results are compared to the respective optimised biasing scenarios obtained by the previously proposed optimisation procedure. With the possibility of sub-pixel resolved measurements of the cluster charge, cluster size and hit efficiency at the beam tests, a detailed investigation of the charge collection within the DEPFET cells at improper biasing was conducted. For these measurements, only a small area of the sensor was illuminated.

Figure 8.27 shows the dependence of the cluster charge MPV, $Q_{\mathrm{MPV}}$, and the hit efficiency $\varepsilon$, averaged over the super-pixels (of size $u \times v=10 \times 8$ pixels), on the depletion voltage for the non-optimal biasing scenario for both DUTs. The decrease in $Q_{\mathrm{MPV}}$ for more negative $V_{\mathrm{HV}}$ in the over-depletion region is in agreement with the measurements presented in Section 7.3. The overall lower $Q_{\mathrm{MPV}}$ for module W40_IF compared to module W11_OF2 is due to a lower DEFPET gate potential of $V_{\text {gate-on }}=-1.6 \mathrm{~V}$ (vs $V_{\text {gate-on }}=-2 \mathrm{~V}$ ). A correlated decrease in hit efficiency is observed. This is expected since a decrease in $Q_{\mathrm{MPV}}$ indicates a shift of the cluster charge distribution to lower values. Equivalently, this can be viewed as an increase of the signal threshold which was shown to degrade the hit efficiency (cf. Fig. 8.23). Figure 8.28 shows the cluster charge 


\section{Measuring Pixel Detector Modules in Beam Tests}

MPV and the hit efficiency as a function of the position on the sensor (per super-pixel) for the different depletion voltages for both DUTs. A non-uniform ring-like decrease in $Q_{\mathrm{MPV}}$ and $\varepsilon$ is observed. The geometry of ring-shaped areas is different between the two DUTs, but are consistent in $Q_{\mathrm{MPV}}$ and $\varepsilon$ for the same DUT. The horizontal stripes visible especially for module W40_IF are artefacts of the LanGau fitting procedure for extracting $Q_{\mathrm{MPV}}$ and are not a property of the device. At the selected depletion voltages, a subpixel analysis is conducted as described in Section 8.7. Figure 8.29 shows the cluster energy MPV and the hit efficiency at sub-pixel resolution for the selected depletion voltages for both DUTs. For some sub-pixel bins, the LanGau fit of the cluster energy distribution to extract $Q_{\mathrm{MPV}}$ does not yield a reliable result (white spots). It should be noted that the overlay in the unit cells is over the full illuminated sensor area and does not distinguish between different ring regions. For a depletion voltage of $V_{\mathrm{HV}}=-54 \mathrm{~V}$, both $Q_{\mathrm{MPV}}$ and $\varepsilon$ are uniform over the sub-pixel bins for both DUTs. When decreasing $V_{\mathrm{HV}}$, inhomogeneities appear with a sub-structure common among pairs of two pixels in $v$-direction (along pixel columns). The observed non-uniformity is not identical in $Q_{\mathrm{MPV}}$ and $\varepsilon$ at a given $V_{\mathrm{HV}}$ on the same DUT. At the same depletion voltage, the observed non-uniformity differs among the two DUTs as well. The difference in cluster energy MPV and hit efficiency sub-structures is explained by the non-linear dependence of the hit efficiency on the applied signal threshold (cf. Fig. 8.23). As discussed before, the decrease in $Q_{\mathrm{MPV}}$ is equivalent to an increase of the threshold. Ultimately, the hit efficiency is the figure of merit for the detector performance. The measurement of the decrease in $Q_{\mathrm{MPV}}$, however, gives insight in the mechanisms of charge collection inefficiencies. Since the DUT W11_OF2 exhibits an overall larger cluster charge MPV (in units of ADU, cf. Fig. 8.27) together with a less steep decrease of the MPV and the hit efficiency for decreasing depletion voltage, the $V_{\mathrm{HV}}$ dependence measured on module W11_OF2 can be considered to effectively measure the evolution in between the $V_{\mathrm{HV}}=-62 \mathrm{~V}$ and $V_{\mathrm{HV}}=-66 \mathrm{~V}$ measurements on module W40_IF (disregarding the different pixel pitches).

With decreasing depletion voltage, the charge collection is degraded (lower $Q_{\mathrm{MPV}}$ and $\varepsilon)$ first at small and localised spots at the borders between columns of pixels. These spots are located approximately at half the pixel pitch in $v$-direction $\left(P_{v} / 2\right)$ and repeat very regularly over the pixel grid. The borders between rows of pixels are initially only marginally affected. For more negative $V_{\mathrm{HV}}$, the spots grow in $v$-direction and merge for every two pixels. In addition, a degradation growing inside at every second row pixel border is observed. A repeating pattern of two pixels in $v$-direction manifests. Ultimately, a central region around the common edge of the two-pixel pattern with largely unaffected $Q_{\mathrm{MPV}}$ and $\varepsilon$ is formed with an extension inside the lower of the two pixels. The origin of this asymmetry is not yet fully understood. Figure 8.30 compares the sub-pixel resolved hit efficiency at $V_{\mathrm{HV}}=-70 \mathrm{~V}$ on module W40_IF to the design of the four-fold DEPFET pixel cells. The design features an asymmetry in the pad contacting the DEPFET gates of the $4 \times 4$ pixel blocks. However, this asymmetry lies within the unaffected central region and is only present for every other pixel column. More likely is an influence of the vertical gate and clear lines connecting the four-row 

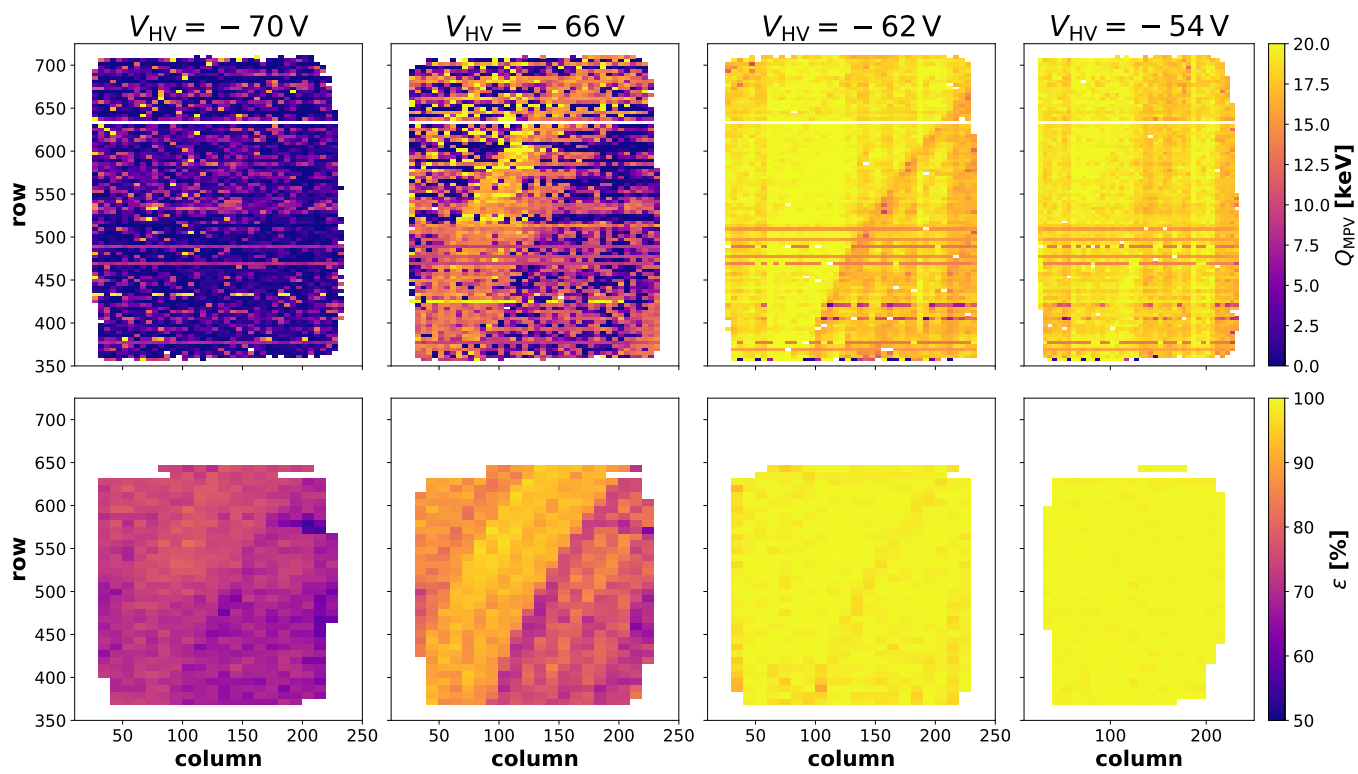

(a) measured on module W40_IF
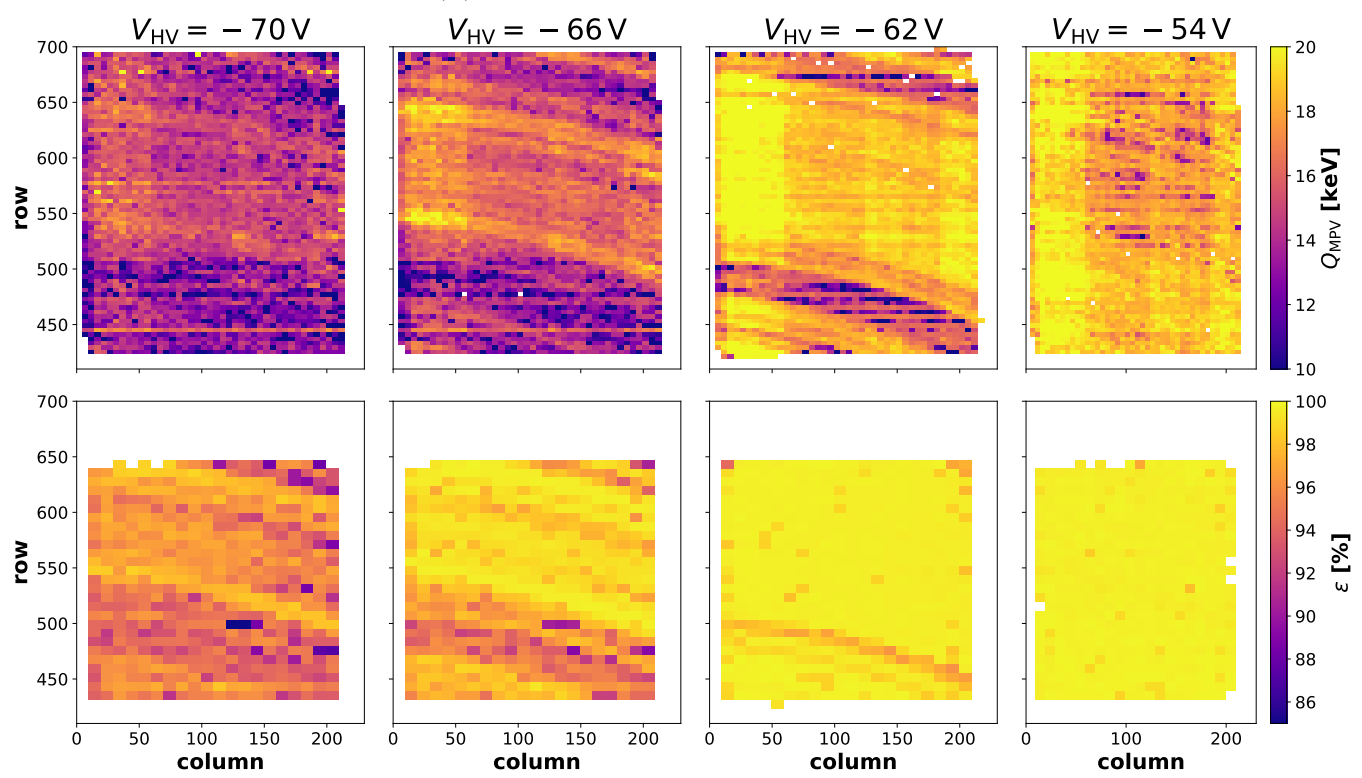

(b) measured on module W11_OF2

Figure 8.28.: Cluster charge MPV $Q_{\mathrm{MPV}}$ and hit efficiency $\varepsilon$ per super-pixel coordinate of the illuminated sensor area for the two DUTs and selected depletion voltages $V_{\mathrm{HV}}$. 


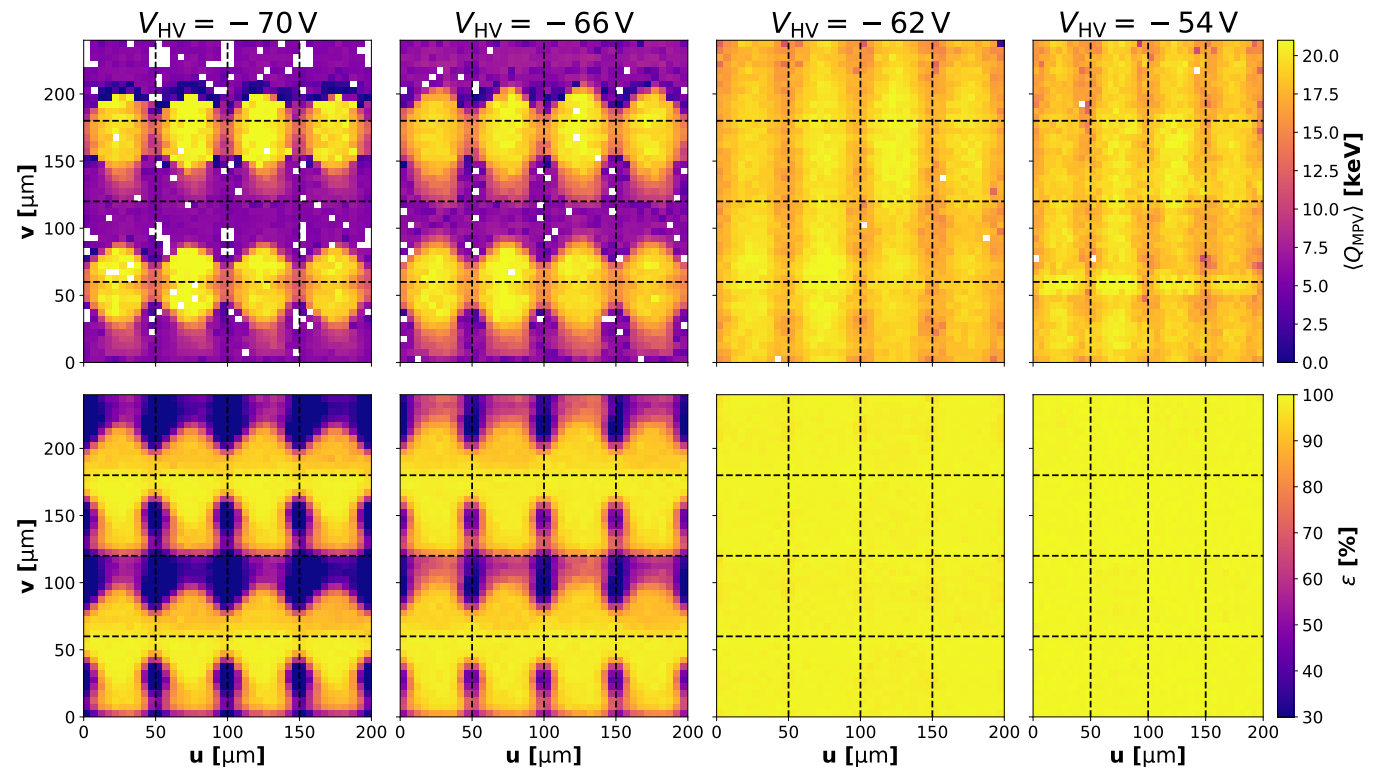

(a) unit cell for pitch $P_{v}=60 \mu \mathrm{m}$ measured on module $\mathrm{W} 40 \_\mathrm{IF}$
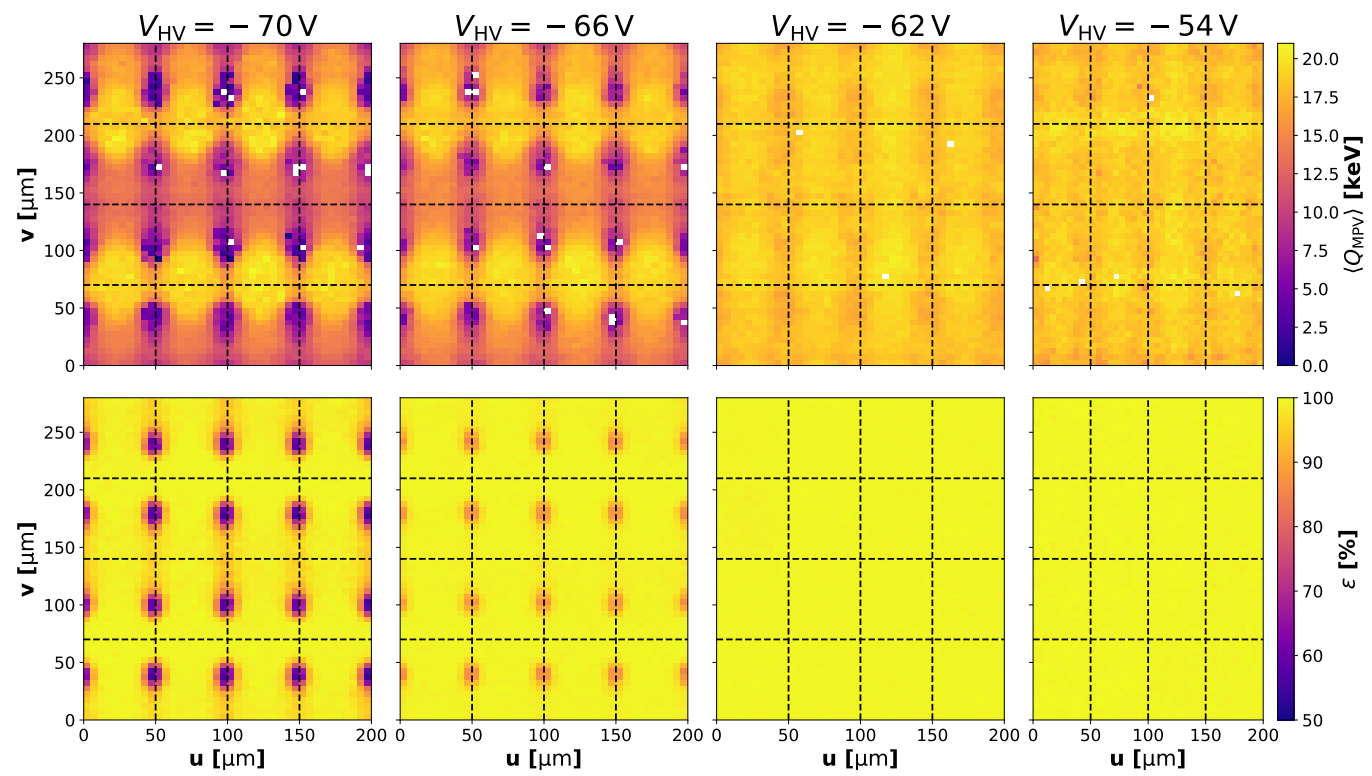

(b) unit cell for pitch $P_{v}=70 \mu \mathrm{m}$ measured on module W11_OF2

Figure 8.29.: Cluster energy MPV, $Q_{\mathrm{MPV}}$, and hit efficiency $\varepsilon$ per sub-pixel bin for two unit cells on the measured DUTs for selected depletion voltages $V_{\mathrm{HV}}$. 

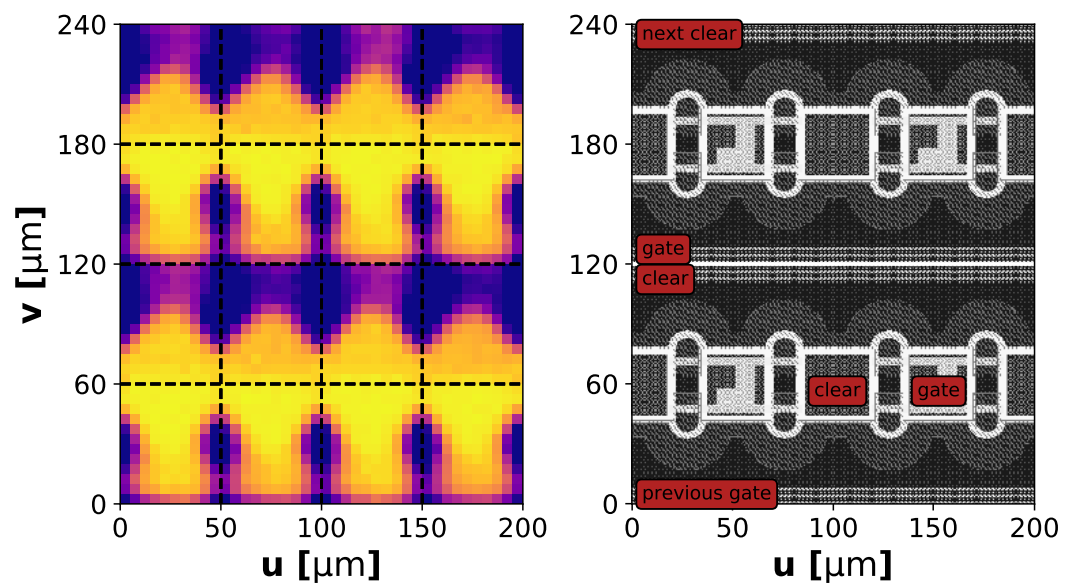

Figure 8.30.: Sub-pixel resolved hit efficiency $\varepsilon$ at a depletion voltage of $V_{\mathrm{HV}}=-70 \mathrm{~V}$ for DUT W40_IF compared to a sketch of the DEPFET pixel design.

of pixels to the respective SWITCHER ASIC outputs on the balcony. More detailed investigations are needed on this issue.

It is demonstrated that a significant, non-uniform degradation of the cluster energy MPV and the hit efficiency, over the sensor area and within the DEPFET pixels, can be avoided by choosing an appropriate depletion voltage. A more stable behaviour, also for varying $V_{\mathrm{HV}}$, can be achieved by optimising the biasing scenario. Figure 8.31 shows the hit efficiency average over all super-pixels for varying depletion voltage for the non-optimal biasing scenario, and the respective DUT's optimised biasing scenario (cf. Tab. 8.2). Evidently, the optimised biasing scenario reduces the dependence of the hit efficiency on $V_{\mathrm{HV}}$.

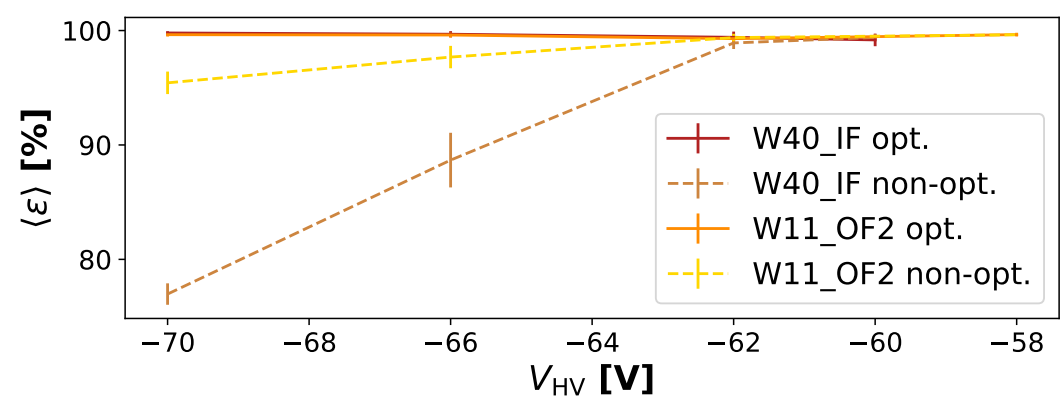

Figure 8.31.: Hit efficiency $\varepsilon$, averaged over all super-pixels, as a function of depletion voltage $V_{\mathrm{HV}}$ for the non-optimal and the optimal biasing scenario for the two DUTs. 

CHAPTER 9

\section{Summary and conclusion}

This thesis presents a thorough study of the characteristics and performance of production modules for the Belle II pixel detector. These DEPFET based detector modules are very complex devices. The characteristics of a single module can be fine tuned in various ways, affecting the digital or analogue read-out, or the properties of the sensor itself. On the other hand, this tunability yields several possible points of failure.

About 60 modules (to account for failures) had to be characterised and optimised to reach a number of 40 well working and well understood candidates for the installation in the Belle II pixel detector in 2019. Due to a delay in the post-characterisation processing of modules, unfortunately, not all candidates could be installed for the start of data taking in 2019. A complete version of the pixel detector will be installed as soon as possible. An in-depth and efficient series testing procedure has been developed and applied to a number of 17 modules in the scope of this thesis. Other collaboration sites contributed as well using a common laboratory setup design and applying the same standardised testing procedure.

The rolling-shutter read-out mode of the DEPFET pixels allows for constructing very thin detector devices, but poses a challenge to the configuration of the front-end read-out electronics. The convoluted interplay of the in-pixel amplification gain, the additional analogue read-out amplification per drain line and the limited dynamic range of the analogue-to-digital conversion makes it difficult to find an optimal tuning of these parameters. Nonetheless, the procedure adopted in the series testing produces consistent characteristics across different modules regarding an acceptable pedestal spread, in-pixel amplification and signal-to-noise ratios. The measured in-pixel amplification values are in very good agreement with the design values of $400 \mathrm{pA} /$ electron to $600 \mathrm{pA} /$ electron. Excellent signal-to-noise ratios in the range of 20 to 40 and even beyond are consistently 


\section{Summary and conclusion}

achieved. The fraction of non-responsive pixels is below $1 \%$ for most of the tested modules. It has been shown that an adequate choice of the DEPFET biasing potentials is crucial to achieve a uniform response to ionising radiation over the sensor. Production variations in the bulk doping concentration cannot be compensated for as their ring-like geometry is different from the column-wise and row-wise tuning of the rollingshutter read-out. A biasing scenario that is largely insensitive to these variations, while maintaining an efficient charge collection, has to be identified. In the scope of the series testing, a feasible biasing optimisation procedure has been adopted and yields consistent results across the tested modules. A grading based on the fraction of non-responsive pixels was established to give a recommendation for the priority of modules to be installed in Belle II.

Detailed investigations of the performance of production modules have been performed in two beam tests. The intrinsic spatial resolution has been measured at perpendicular and inclined particle incidence on the sensor area. The measured incidence angles cover approximately the range that the modules will be exposed to in Belle II. Especially at inclined incidence, the effect of charge sharing between neighbouring pixels can significantly improve the intrinsic spatial resolution of the device. A position finding algorithm based on cluster shapes and relative signal distribution within a cluster models the charge sharing characteristics more precisely than the simple centre of gravity approach. At perpendicular particle incidence, intrinsic spatial resolutions of $8 \mu \mathrm{m}$ to $20 \mu \mathrm{m}$ were measured, dependent on the pixel pitch, cluster sizes and the employed position finding algorithm. For an incidence angle of about $30^{\circ}$, the resolution can be improved to values of $6 \mu \mathrm{m}$ to $7 \mu \mathrm{m}$. The improved position finding algorithm consistently yields better intrinsic spatial resolutions. The observed resolutions are compatible with the expectations from simulations and therefore the Belle II requirements to the pixel detector modules are met. This is also true for the measured hit efficiencies. The modules can be operated at thresholds of about 500 to 1000 electrons, at a MIP signal of about 5500 electrons, and a degradation below a hit efficiency of $98 \%$ is only expected for thresholds of about 2000 electrons. On average, the modules exhibit a reasonable hit efficiency of $99.6 \%$ and the efficiency is uniform over the DEPFET pixel matrix and over the geometrical extent of the individual pixels. This is in agreement with, and even slightly above, the value of about $98 \%$ reported from the first operational experience. With the excellent pointing resolution provided by the beam telescope, a sub-pixel study of the hit efficiency was performed. The results show a homogeneous hit efficiency across the $4 \times 4$ pixel unit cells. This confirms that charges are collected reliably and efficiently over the full pixel area. For an optimised biasing scenario, the charge collection efficiency, measured by the sub-pixel resolved cluster charge MPV and the hit efficiency, is uniform and stable also for varying depletion voltage. At a non-optimal biasing scenario, severe degradations are observed for decreasing depletion voltage. The decrease in charge collection efficiency is non-uniform over the DEPFET cells and the simulated "over-depletion" effect is supported by these measurements.

The findings for the module performance are also valid for modules that received a total 
ionising dose at the DEPFET silicon oxides of $1 \mathrm{kGy}$ and $266 \mathrm{kGy}$, respectively. Both modules are still functional and exhibit very similar characteristics compared to nonirradiated modules regarding read-out noise, signal-to-noise values, in-pixel amplification gains and hit efficiencies.

Overall, a clear optimisation procedure is available for operating the pixel detector modules. The characteristics are consistent across modules and the observed performance, in the laboratory and in beam test measurements, is in agreement with the requirements for the Belle II experiment. 

[1] J. J. Thomson, XL. Cathode Rays, The London, Edinburgh, and Dublin Philosophical Magazine and Journal of Science 44 (1897) 293-316.

[2] S. H. Neddermeyer and C. D. Anderson, Note on the Nature of Cosmic-Ray Particles, Phys. Rev. 51 (1937) 884-886.

[3] M. L. Perl, et al., Evidence for Anomalous Lepton Production in $e^{+}-e^{-}$ Annihilation, Phys. Rev. Lett. 35 (1975) 1489-1492.

[4] P. Zyla et al., Review of Particle Physics, Prog. Theor. Exp. Phys. 2020 (2020), $083 \mathrm{C} 01$.

[5] LHCb collaboration, Observation of structure in the $J / \Psi$-pair mass spectrum, Sci. Bull. 65 (2020) 1983-1993.

[6] R. Aaij, et al., Observation of a Narrow Pentaquark State, $P_{c}(4312)^{+}$, and of the Two-Peak Structure of the $P_{c}(4450)^{+}$, Phys. Rev. Lett. 122 (2019).

[7] Z. Maki, M. Nakagawa, and S. Sakata, Remarks on the Unified Model of Elementary Particles, Prog. Theor. Phys. 28 (1962) 870-880.

[8] R. Davis, D. S. Harmer, and K. C. Hoffman, Search for Neutrinos from the Sun, Phys. Rev. Lett. 20 (1968) 1205-1209.

[9] Y. Fukuda, et al., Evidence for Oscillation of Atmospheric Neutrinos, Phys. Rev. Lett. 81 (1998) 1562-1567.

[10] C. S. Wu, et al., Experimental Test of Parity Conservation in Beta Decay, Phys. Rev. 105 (1957) 1413-1415.

[11] N. Cabibbo, Unitary Symmetry and Leptonic Decays, Phys. Rev. Lett. 10 (1963) 531-533. 


\section{BIBLIOGRAPHY}

[12] J. H. Christenson, et al., Evidence for the $2 \pi$ Decay of the $K_{2}^{0}$ Meson, Phys. Rev. Lett. 13 (1964) 138-140.

[13] V. Fanti, et al., A new measurement of direct CP violation in two pion decays of the neutral kaon, Phys. Lett. B 465 (1999) 335-348.

[14] A. Alavi-Harati, et al., Observation of Direct CP Violation in $K_{S, L} \rightarrow \pi \pi$ Decays, Phys. Rev. Lett. 83 (1999) 22-27.

[15] M. Kobayashi and T. Maskawa, CP-Violation in the Renormalizable Theory of Weak Interaction, Prog. Theor. Phys. 49 (1973) 652-657.

[16] M. Tanabashi, et al., Review of Particle Physics, Phys. Rev. D 98 (2018).

[17] A. Moll, Comprehensive study of the background for the Pixel Vertex Detector at Belle II. PhD thesis, Ludwig-Maximilians-Universität München, 2015.

[18] A. J. Bevan, et al., The Physics of the B Factories, Eur. Phys. J. C 74 (2014) 3026.

[19] K. Akai, K. Furukawa, and H. Koiso, SuperKEKB collider, Nucl. Instrum. Meth. A 907 (2018) 188-199.

[20] The ATLAS Collaboration, et al., The ATLAS Experiment at the CERN Large Hadron Collider, J. Instrum. 3 (2008) S08003-S08003.

[21] The CMS Collaboration, et al., The CMS experiment at the CERN LHC, J. Instrum. 3 (2008) S08004-S08004.

[22] G. Aad, et al., Observation of a new particle in the search for the Standard Model Higgs boson with the ATLAS detector at the LHC, Phys. Lett. B 716 (2012) 1-29.

[23] S. Chatrchyan, et al., Observation of a new boson at a mass of $125 \mathrm{GeV}$ with the CMS experiment at the LHC, Phys. Lett. B 716 (2012) 30-61.

[24] V. A. M. and, SUSY searches in ATLAS and CMS, in Proceedings of Corfu Summer Institute 2019 "School and Workshops on Elementary Particle Physics and Gravity" - PoS(CORFU2019). PoS, Aug, 2020.

[25] The LHCb Collaboration, et al., The LHCb Detector at the LHC, J. Instrum. 3 (2008) S08005-S08005.

[26] Heavy Flavor Averaging Group, et al., Averages of b-hadron, c-hadron, and $\tau$-lepton properties as of 2018, arxiv:1909.12524.

[27] R. Aaij, et al., Test of lepton universality with $B^{0} \rightarrow K^{* 0} l^{+} l^{-}$decays, J. High Energy Phys. 2017 (2017). 
[28] R. Aaij, et al., Measurement of the Ratio of the $B^{0} \rightarrow D^{*-} \tau^{+} \nu_{\tau}$ and $B^{0} \rightarrow D^{*-} \mu^{+} \nu_{\mu}$ Branching Fractions Using Three-Prong $\tau$-Lepton Decays, Phys. Rev. Lett. 120 (2018).

[29] T. Abe, et al., Belle II Technical Design Report, 1011.0352.

[30] E. Kou, et al., The Belle II Physics Book, Prog. Theor. Exp. Phys. 2019 (2019), $123 \mathrm{C} 01$.

[31] P. A. Čerenkov, Visible Radiation Produced by Electrons Moving in a Medium with Velocities Exceeding that of Light, Phys. Rev. 52 (1937) 378-379.

[32] J. Charles, et al., New physics in B meson mixing: Future sensitivity and limitations, Phys. Rev. D 102 (2020) 056023.

[33] M. Thomson, Modern Particle Physics. Cambridge University Press, Sep, 2013.

[34] J. P. Lees, et al., Evidence for an Excess of $\bar{B} \rightarrow D^{(*)} \tau^{-} \bar{\nu}_{\tau}$ Decays, Phys. Rev. Lett. 109 (2012).

[35] M. Huschle, et al., Measurement of the branching ratio $\bar{B} \rightarrow D^{(*)} \tau^{-} \bar{\nu}_{\tau}$ relative $\bar{B} \rightarrow D^{(*)} l^{-} \bar{\nu}_{l}$ decays with hadronic tagging at Belle, Phys. Rev. D 92 (2015) 072014 .

[36] R. Aaij, et al., Measurement of the Ratio of Branching Fractions $\mathcal{B}\left(\bar{B}^{0} \rightarrow D^{*+} \tau^{-} \bar{\nu}_{\tau}\right) / \mathcal{B}\left(\bar{B}^{0} \rightarrow D^{*+} \mu^{-} \bar{\nu}_{\mu}\right)$, Phys. Rev. Lett. 115 (2015).

[37] V. Keus, et al., CP violating Two-Higgs-Doublet Model: constraints and LHC predictions, J. High Energy Phys. 2016 (2016) 1-24.

[38] I. Esteban, et al., The fate of hints: updated global analysis of three-flavor neutrino oscillations, J. High Energy Phys. 2020 (2020).

[39] T. Bilka, Belle II Vertex Detector Performance, in Proceedings of The 28th International Workshop on Vertex Detectors - PoS(Vertex2019). Sissa Medialab, Feb, 2020.

[40] K. Kleinknecht, Detektoren für Teilchenstrahlung. Vieweg+Teubner Verlag, 2005.

[41] U. Stolzenberg, Radiation length measurements with high-resolution telescopes. $\mathrm{PhD}$ thesis, Georg-August-Universität Göttingen, July, 2019. http://cds.cern.ch/record/2690257.

[42] L. Rossi, et al., Pixel Detectors. Springer-Verlag GmbH, 2006.

[43] J. Kemmer and G. Lutz, New detector concepts, Nucl. Instrum. Meth. A 253 (1987) 365-377. 


\section{BIBLIOGRAPHY}

[44] M. Koch, Development of a Test Environment for the Characterization of the Current Digitizer Chip DCD2 and the DEPFET Pixel System for the Belle II Experiment at SuperKEKB. Phd thesis, Rheinische Friedrich-Wilhelms-Universität Bonn, Sept., 2011. CERN-THESIS-2011-084.

[45] L. Reuen, et al., Performance of a DEPFET prototype module for the ILC vertex detector, IEEE Trans. Nucl. Sci. 53 (2006) 1719-1725.

[46] L. Bombelli, C. Fiorini, and M. Porro, Fast DEPFET current readout for X-ray astronomy missions, Nucl. Instrum. Meth. A 604 (2009) 679-683.

[47] L. Andricek, et al., Processing of ultra-thin silicon sensors for future $e^{+} e^{-}$linear collider experiments, IEEE Trans. Nucl. Sci. 51 (2004) 1117-1120.

[48] H. Schreeck, et al., Effects of gamma irradiation on DEPFET pixel sensors for the Belle II experiment, Nucl. Instrum. Meth. A 959 (2020) 163522.

[49] P. Fischer, et al., Readout concepts for DEPFET pixel arrays, Nucl. Instrum. Meth. A 512 (2003) 318-325.

[50] I. Peric, et al., DCDB and SWITCHERB, the readout ASICS for belle II DEPFET pixel detector, in IEEE Nucl. Sci. Conf. R. IEEE, Oct, 2011.

[51] F. J. Lütticke, Development of Pixel Modules for the Belle II Detector. PhD thesis, Rheinische Friedrich-Wilhelms-Universität Bonn, 2019. https : //bonnus.ulb.uni-bonn.de/SummonRecord/FETCH-bonn_catalog_44541092.

[52] I. Peric, P. Fischer, and C. Kreidl, DCD-Bv4-Pipeline Reference Manual, Document revision 1 (2015).

[53] DEPFET collaboration, internal documentation and private communication,.

[54] B. Spruck, et al., Belle II Pixel Detector Commissioning and Operational Experience, in Proceedings of The 28th International Workshop on Vertex Detectors - PoS(Vertex2019). Sissa Medialab, Mar, 2020.

[55] P. Gomis, Development of the Pixel Detector for the Belle II experiment and top quark mass measurement in radiative events at the future electron-positron linear collider. PhD thesis, Universitat de Valencia, Jan., 2020.

http://hdl. handle.net/10261/224085.

[56] S. Rummel, The power supply system for the DEPFET pixel detector at BELLE II, Nucl. Instrum. Meth. A 699 (2013) 51-55.

[57] D. Levit, I. Konorov, and S. Paul, FPGA based data read-out system of the Belle II pixel detector, in 2014 19th IEEE-NPSS Real Time Conference. IEEE, May, 2014. 
[58] M. Ritzert, Status of the EPICS-Based Control and Interlock System of the Belle II PXD, Proceedings of the 15th Int. Conf. on Accelerator and Large Experimental Physics Control Systems ICALEPCS2015 (2015).

[59] P. Wieduwilt, Optimization of the front-end read-outel ectronics for the Belle II DEPFET Sensor, Master's thesis, Georg-August-Universität Göttingen, Aug., 2016. II.Physik-UniGö-MSc-2016/04.

[60] E. Browne, Nuclear Data Sheets for A = 90, Nucl. Data Sheets 82 (1997) 379-546.

[61] S. Agostinelli, et al., Geant4 - a simulation toolkit, Nucl. Instrum. Meth. A 506 (2003) 250-303.

[62] H. Schreeck, Commissioning and first data taking experience with the Belle II pixel vertex detector. $\mathrm{PhD}$ thesis, Georg-August-Universität Göttingen, May, 2020. http://hdl. handle.net/21.11130/00-1735-0000-0005-13F7-F.

[63] B. Bergbauer, Study of macroscopic and microscopic homogeneity of DEPFET $X$-ray detectors. PhD thesis, Technische Universität Chemnitz, June, 2015.

[64] F. Müller, Characterization and optimization of the prototype DEPFET modules for the Belle II Pixel Vertex Detector. PhD thesis, Ludwig-Maximilians-Universität München, July, 2017. https://nbn-resolving org/urn:nbn:de:bvb:19-210714.

[65] R. Diener, et al., The DESY II test beam facility, Nucl. Instrum. Meth. A 922 (2019) 265-286.

[66] P. Wieduwilt, et al., Performance of production modules of the Belle II pixel detector in a high-energy particle beam, Nucl. Instrum. Meth. A 991 (2021) 164978.

[67] I. Rubinskiy, An EUDET/AIDA Pixel Beam Telescope for Detector Development, Physics Procedia 37 (2012) 923-931.

[68] J. Behr, Jets at High $Q^{2}$ at HERA and Test Beam Measurements with the EUDET Pixel Telescope. PhD thesis, FB Physik, Universtät Hamburg, Sept., 2010. http://www-library.desy.de/cgi-bin/showprep.pl?desy-thesis-10-038. DESY-THESIS-10-038.

[69] C. Hu-Guo, et al., First reticule size MAPS with digital output and integrated zero suppression for the EUDET-JRA1 beam telescope, Nucl. Instrum. Meth. A 623 (2010) 480-482.

[70] H. Jansen, et al., Performance of the EUDET-type beam telescopes, EPJ Techn. Instrum. 3 (2016) 7.

[71] P. Ahlburg, et al., EUDAQ - a data acquisition software framework for common beam telescopes, J. Instrum. 15 (2020) P01038-P01038. 


\section{BIBLIOGRAPHY}

[72] M. Garcia-Sciveres, et al., The FE-I4 pixel readout integrated circuit, Nucl. Instrum. Meth. A 636 (2011) S155-S159.

[73] T. Obermann, et al., Implementation of a configurable FE-I4 trigger plane for the AIDA telescope, J. Instrum. 9 (2014) C03035-C03035.

[74] B. Schwenker et al., Test Beam Software Framework, 2021. https://bitbucket.org/testbeam/tbsw. Retrieved on 01.03.2021.

[75] B. Schwenker, Development and validation of a model for the response of the Belle II vertex detector. $\mathrm{PhD}$ thesis, Georg-August-Universität Göttingen, Oct., 2014. http://hdl . handle.net/11858/00-1735-0000-0023-992D-9.

[76] R. Frühwirth, Application of Kalman filtering to track and vertex fitting, Nucl. Instrum. Meth. A 262 (1987) 444-450.

[77] V. Karimaki, et al., Sensor Alignment by Tracks, physics/0306034.

[78] R. Turchetta, Spatial resolution of silicon microstrip detectors, Nucl. Instrum. Meth. A 335 (1993) 44-58.

[79] L. Andricek, et al., Intrinsic resolutions of DEPFET detector prototypes measured at beam tests, Nucl. Instrum. Meth. A 638 (2011) 24-32.

[80] R. E. Kalman, A New Approach to Linear Filtering and Prediction Problems, J. Basic Eng. 82 (1960) 35-45.

[81] E. J. Wolin and L. L. Ho, Covariance matrices for track fitting with the Kalman filter, Nucl. Instrum. Meth. A 329 (1993) 493-500.

[82] R. Frühwirth, T. Todorov, and M. Winkler, Estimation of detector alignment parameters using the Kalman filter with annealing, J. Phys. G Nucl. Partic. 29 (2003) 561-574.

[83] M. Dragicevic, et al., Test beam performance measurements for the Phase I upgrade of the CMS pixel detector, J. Instrum. 12 (2017) P05022-P05022. 
Appendices 

The hit efficiency $\varepsilon$ is computed as a function of the position on the sensor in super-pixel bins of size $u \times v=10 \times 8$ pixels. The following figures show the hit efficiency maps and distribution for all measured DUTs.
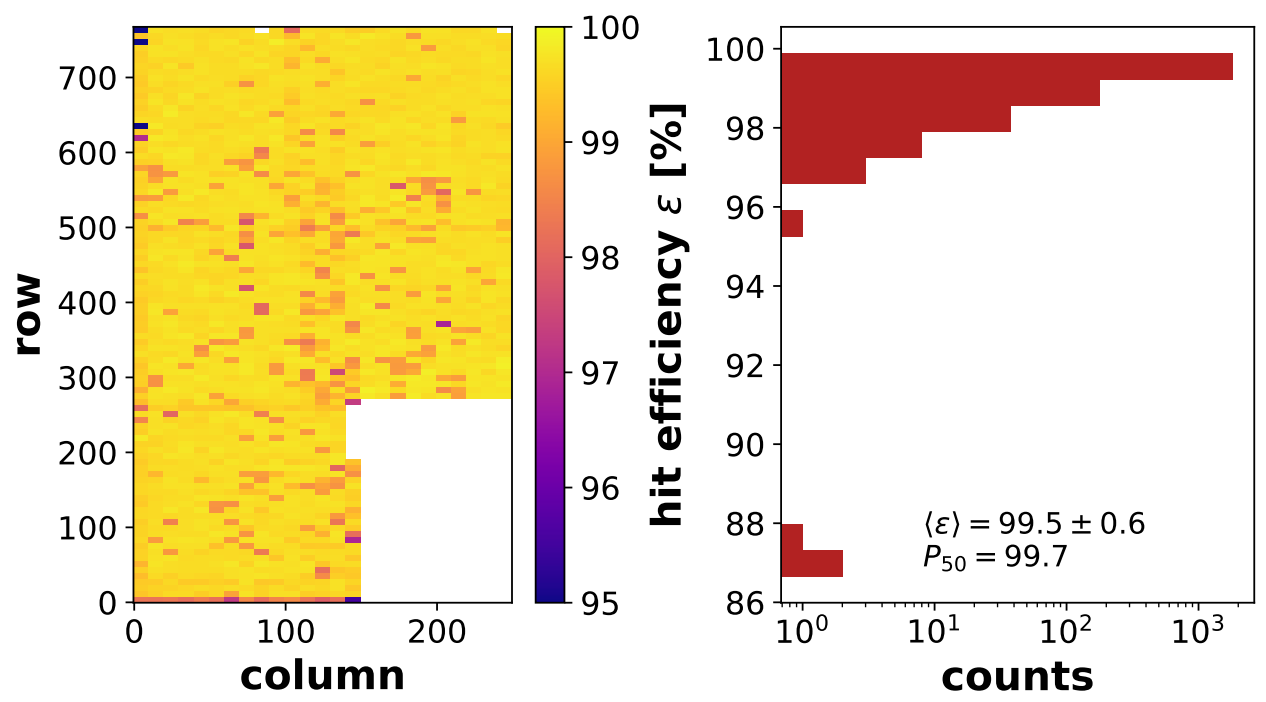

Figure A.1.: Hit efficiency $\varepsilon$ as a function of the super-pixel coordinate and distribution over all super-pixels for DUT W40_IF. 

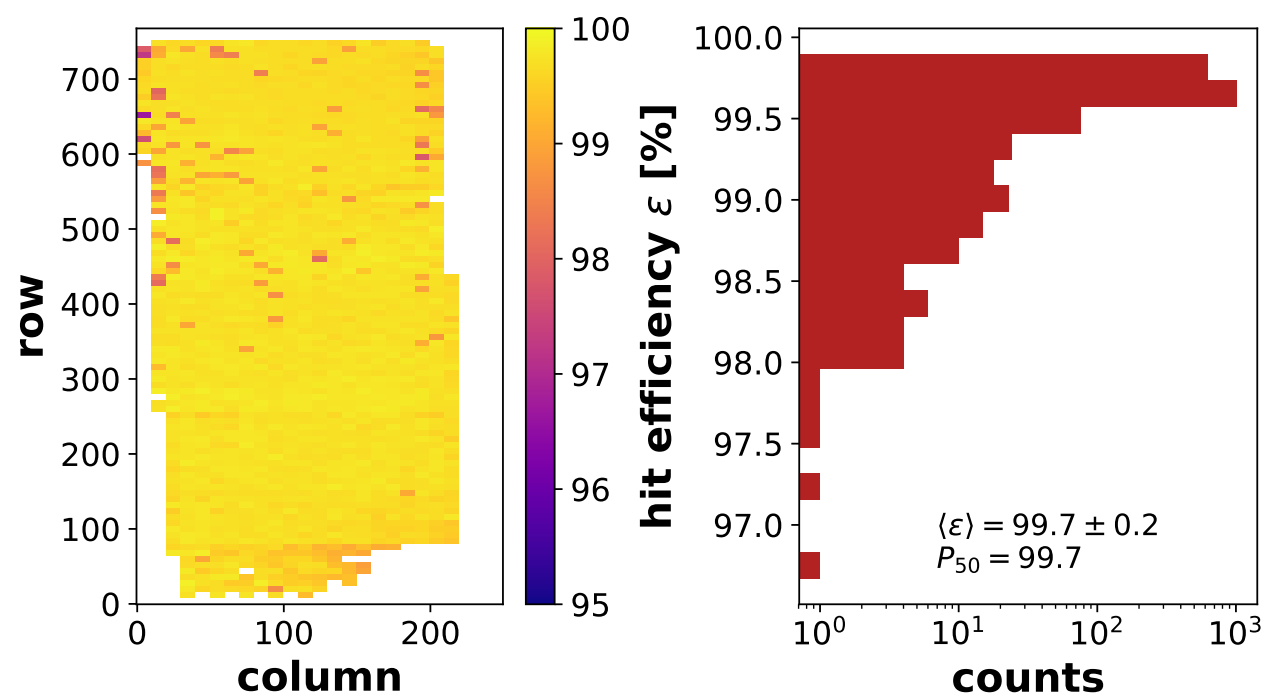

Figure A.2.: Hit efficiency $\varepsilon$ as a function of the super-pixel coordinate and distribution over all super-pixels for DUT W11_OF2.
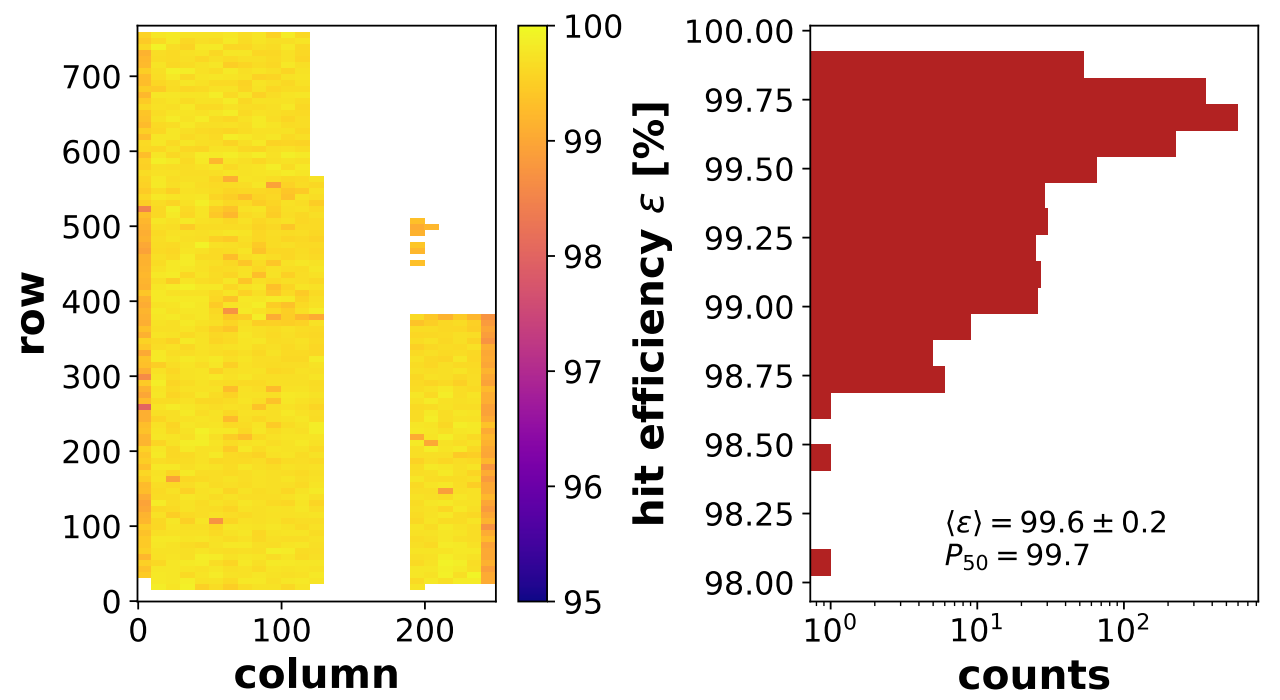

Figure A.3.: Hit efficiency $\varepsilon$ as a function of the super-pixel coordinate and distribution over all super-pixels for DUT W05_OB1. 

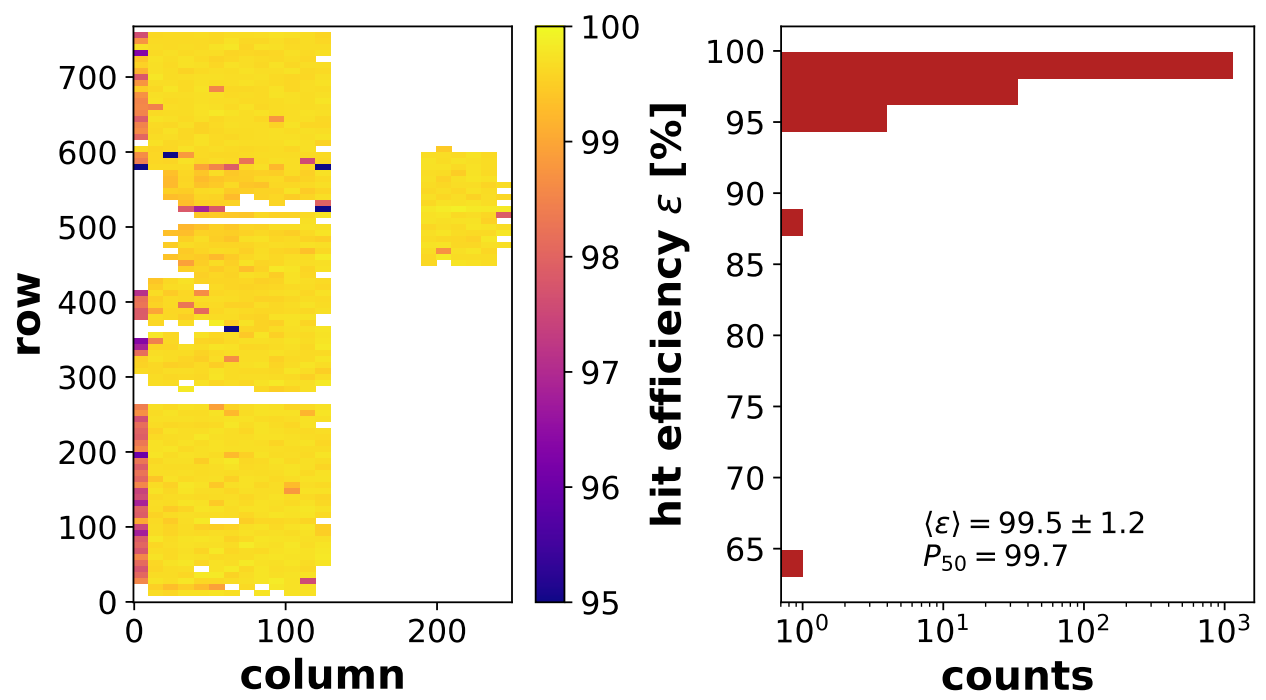

Figure A.4.: Hit efficiency $\varepsilon$ as a function of the super-pixel coordinate and distribution over all super-pixels for DUT W05_OB1 ${ }^{\gamma}$.
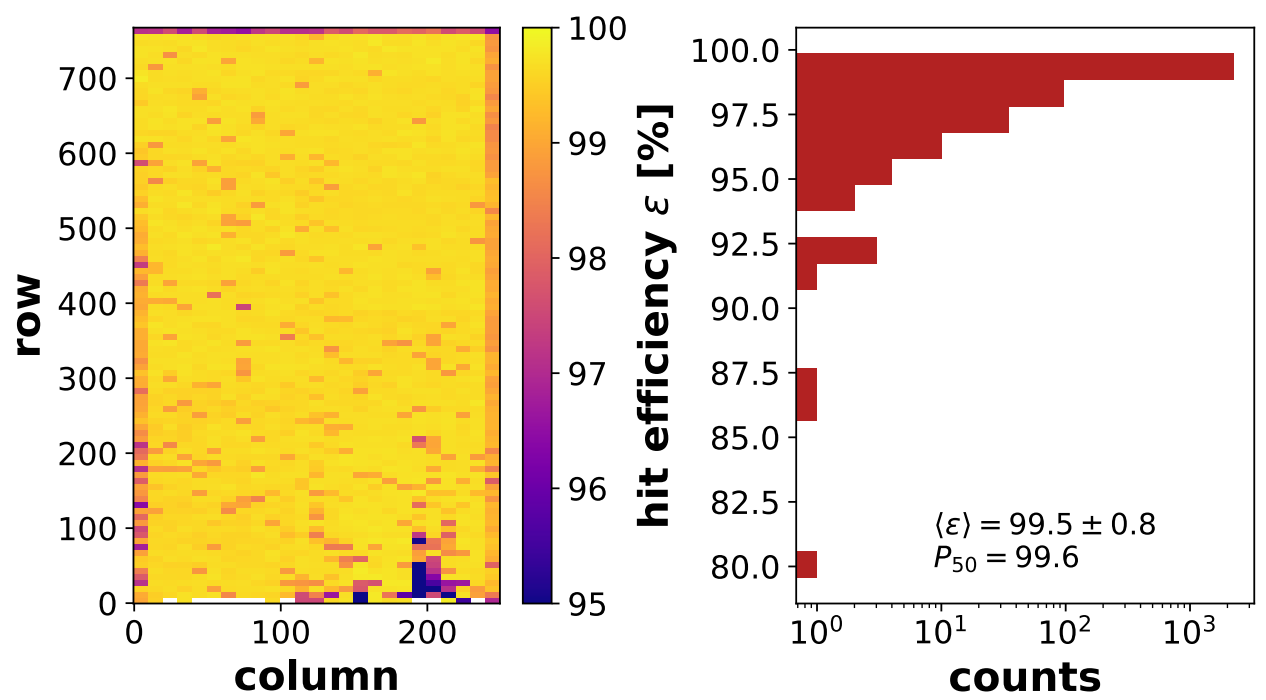

Figure A.5.: Hit efficiency $\varepsilon$ as a function of the super-pixel coordinate and distribution over all super-pixels for DUT W37_OF1. 



\section{APPENDIX B}

\section{DCD input gain dependence}

The DCD input gain $g_{\mathrm{DCD}}$ directly influences the overall pedestal spread. Figure B.1 shows the dependence of the pedestal spread and the pixel fluctuation (noise) as a function of the DCD gain. The noise is mostly unaffected, indicating that the major contribution to the read-out noise is from the analogue-digital conversion in the DCD.
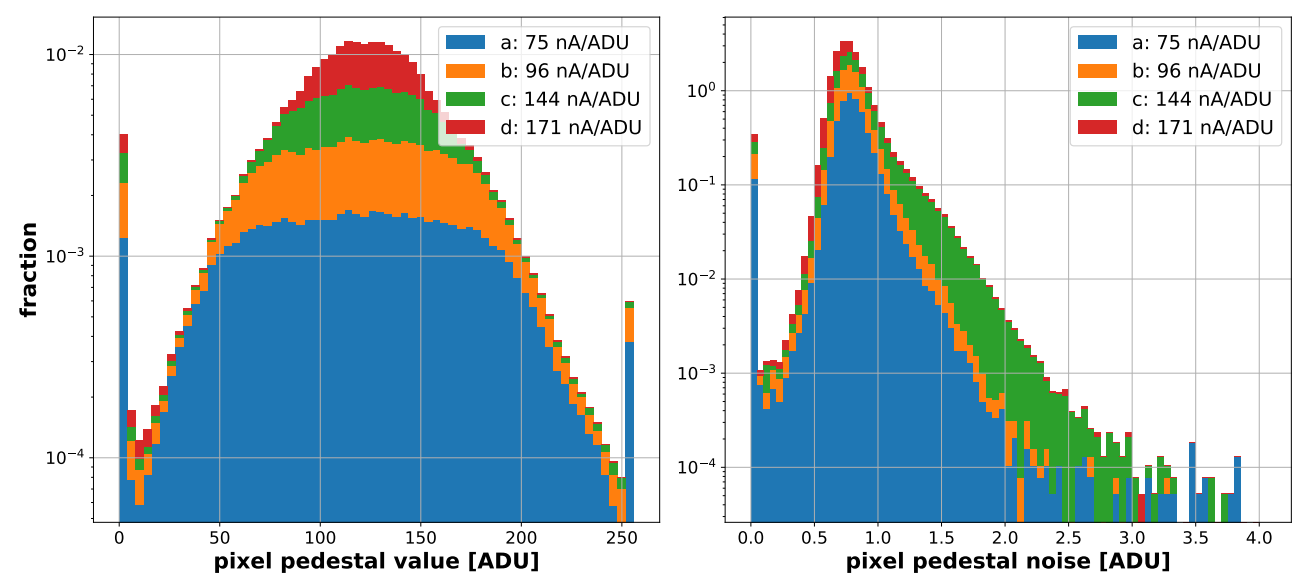

Figure B.1.: Pedestal distribution and pedestal noise distribution as a function of the selectable DCD input gain $g_{\mathrm{DCD}}$. 



\section{Acknowledgements}

I would like to express my sincere gratitude to Prof. Dr. Ariane Frey for the opportunity to conduct this dissertation project in her working group and for being a supporting and encouraging supervisor over the course of the last five years. I would like to thank PD Dr. Jörn Große-Knetter for being the co-referee of this thesis. I thank the further members of the examination board Prof. Dr. Stan Lai, Prof. Dr. Hans Christian Hofsäss, Prof. Dr. Baida Achkar and Prof. Dr. Wolfram Kollatschny.

I would like to thank as well Dr. Benjamin Schwenker for supervising my work, providing insightful comments and suggestions and for lively discussions. The measurements and analyses conducted in the scope of this thesis benefited widely from his inputs. For very fruitful discussions and successful and agreeable scientific collaboration, I would like to thank all members of the DEPFET, PXD and Belle II collaborations. I am especially grateful to Dr. Harrison Schreeck and Botho Paschen for their scientific contributions and the enjoyable collaboration and time spent together at measurement campaigns, workshops and conferences. Special thanks go to my office co-workers and members of the working group, Dr. Ulf Stolzenberg and Uwe Gebauer. A warm thanks for constructive discussions and a pleasant working environment goes to all current and past members of the working group. The measurements leading to parts of the results presented in this thesis have been performed at the Test Beam Facility at DESY Hamburg (Germany), a member of the Helmholtz Association (HGF).

Mein besonderer und tief empfundener Dank geht an meine Familie und Freunde für unermüdliche Unterstützung und Ermutigung über die letzten Jahre. Allen voran geht dieser Dank an meine Eltern, meine Geschwister und meine Großeltern für fortwährenden Rückhalt und Kraft und unbeirrbare Zuversicht. Max danke ich für Rat und Beistand. Philipp danke ich für Ablenkung und Humor. Ohne Euch wäre ich nicht so weit gekommen. 
